\title{
Synthesis of Spiro- $\beta$-lactam-pyrroloquinolines as Fused Heterocyclic Scaffolds through Post-transformation Reactions
}

\author{
Farhad Golmohammadi ${ }^{\mathrm{a}}$, Saeed Balalaie*a,b, Vaezeh Fathi Vavsaria ${ }^{\mathrm{a}}$, Muhammad U. Anwar ${ }^{\mathrm{c}}$, \\ Ahmed Al-Harrasi*c \\ ${ }^{a}$ Peptide Chemistry Research Center, K. N. Toosi University of Technology, P. O. Box 15875-4416, \\ Tehran,Iran, balalaie@ kntu.ac.ir,Tel:+98-21-23064226, Fax: +98-21-22889403 \\ ${ }^{b}$ Medical Biology Research Center, Kermanshah University of Medical Sciences, Kermanshah, Iran \\ ${ }^{c}$ Natural and Medical Sciences Research Center, University of Nizwa, P.O. Box 33, Postal Code 616, \\ Birkat Al Mauz, Nizwa, Sultanate of Oman
}

\section{Table of Contents}

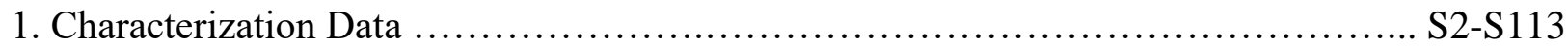

2. X-ray crystallographic analysis for compound $\mathbf{6 b}$ S114-S116

3. References S117 


\section{Characterization Data}

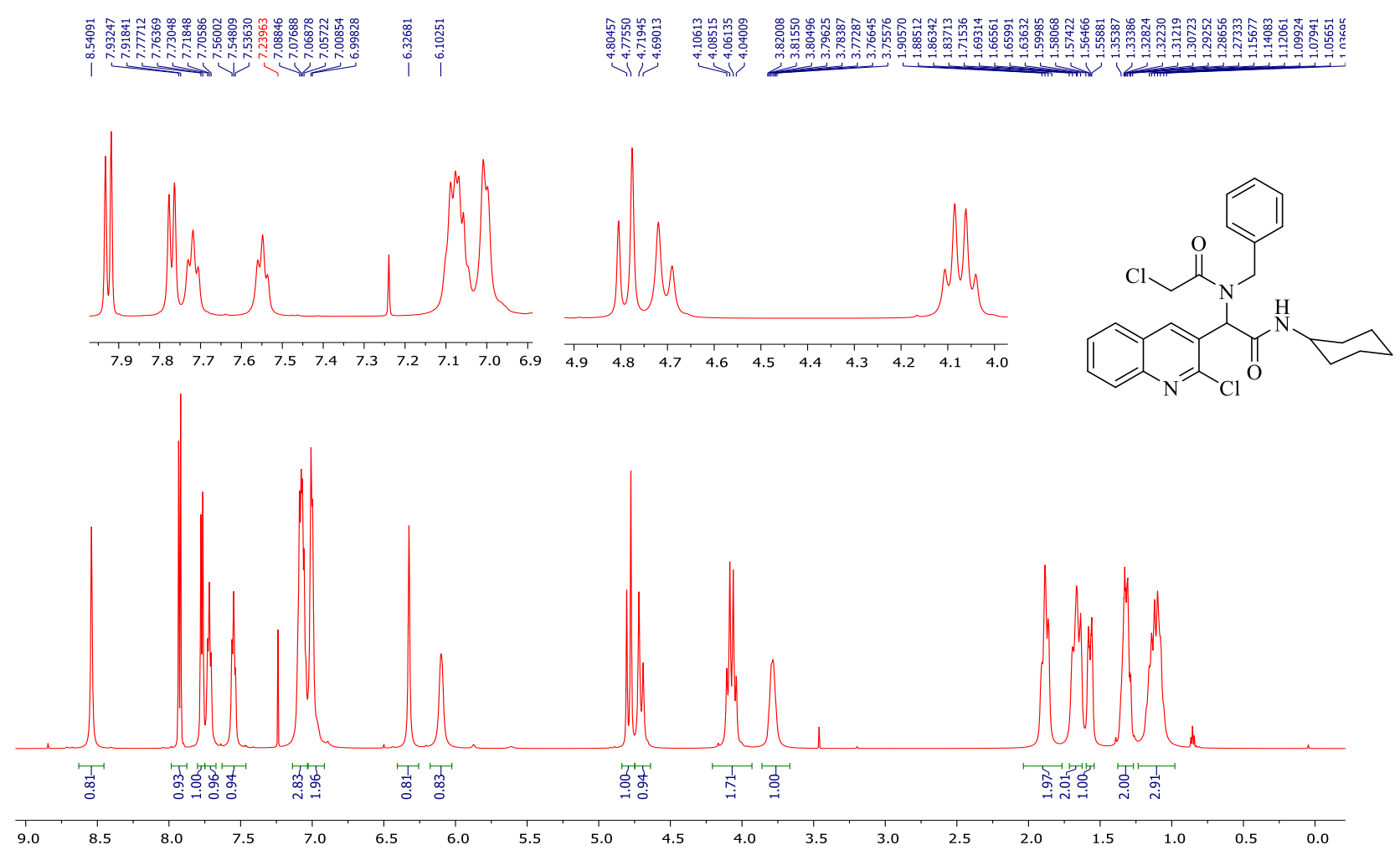

Figure S1: ${ }^{1} \mathrm{H}-\mathrm{NMR}$ of compound $\mathbf{5 a}\left(600 \mathrm{MHz}, \mathrm{CDCl}_{3}\right)$
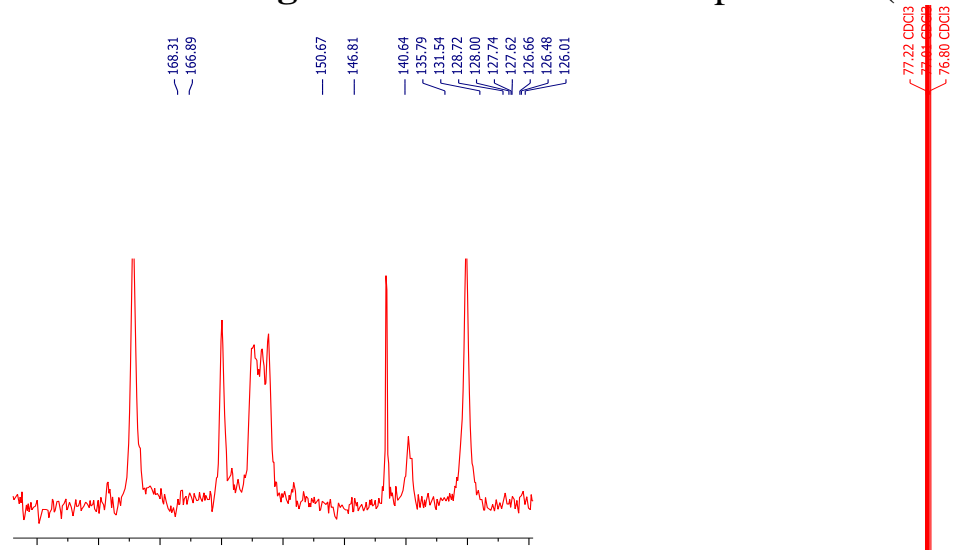

隹

\begin{tabular}{lllllllllll}
\hline 129.5 & 129.0 & 128.5 & 128.0 & 127.5 & 127.0 & 126.5 & 126.0 & 125.5
\end{tabular}
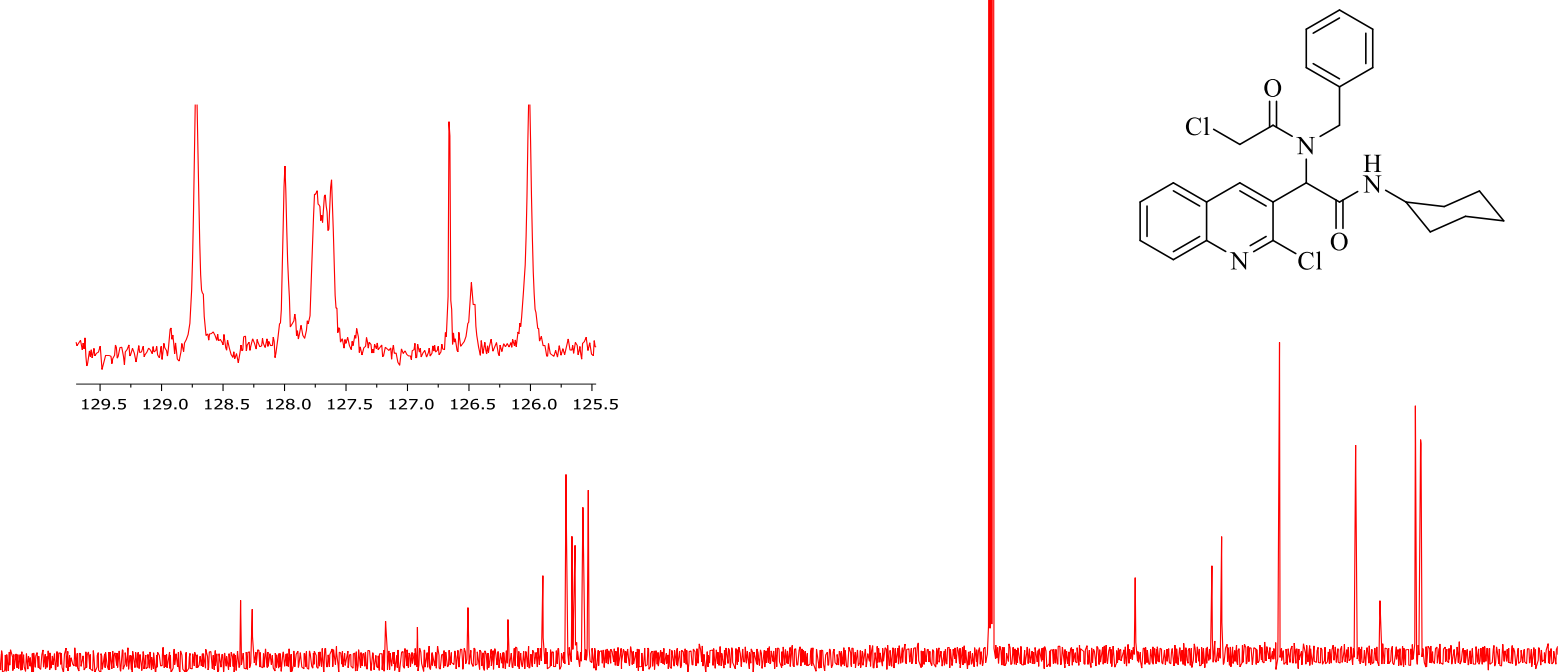

Figure S2: ${ }^{13} \mathrm{C}-\mathrm{NMR}$ of compound $\mathbf{5 a}\left(150 \mathrm{MHz}, \mathrm{CDCl}_{3}\right)$ 


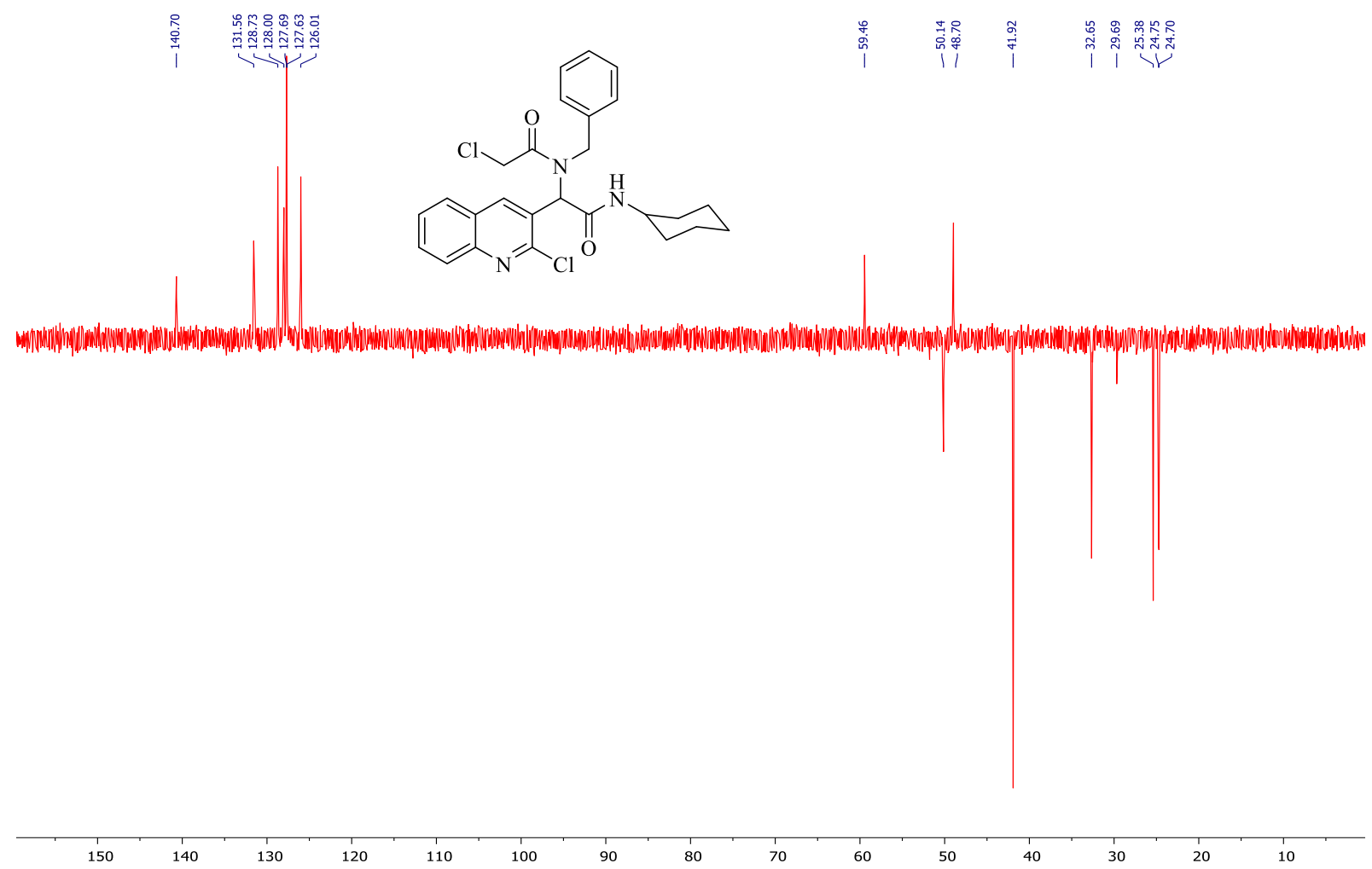

Figure S3: ${ }^{13} \mathrm{C}-\mathrm{NMR}$ (DEPT 135) of compound $\mathbf{5 a}\left(150 \mathrm{MHz}, \mathrm{CDCl}_{3}\right)$

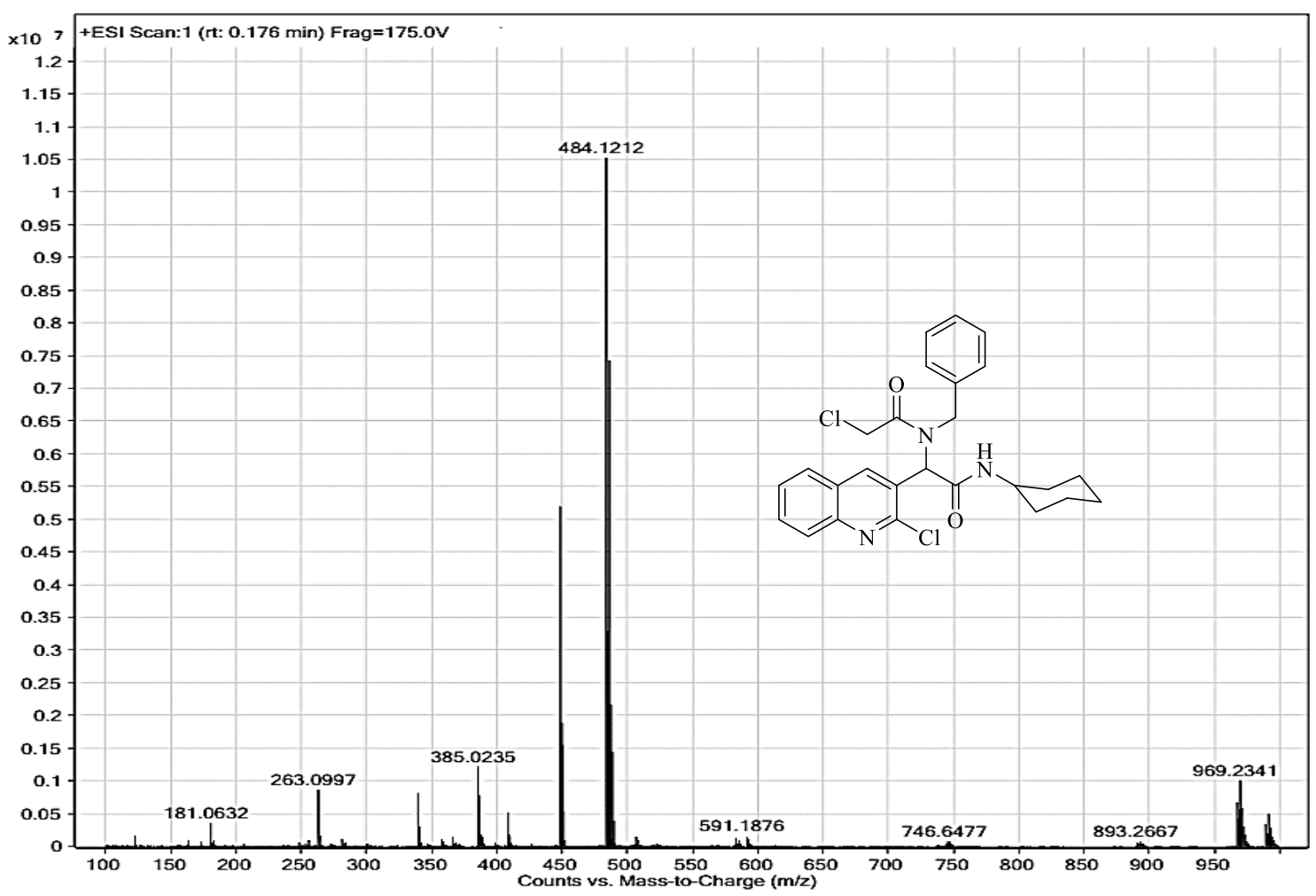

Figure S4: HRMS-ESI of 5a with formula $\mathrm{C}_{26} \mathrm{H}_{27} \mathrm{Cl}_{2} \mathrm{~N}_{3} \mathrm{O}_{2}$ and $[\mathrm{M}+\mathrm{H}]^{+} 484.1223$ 


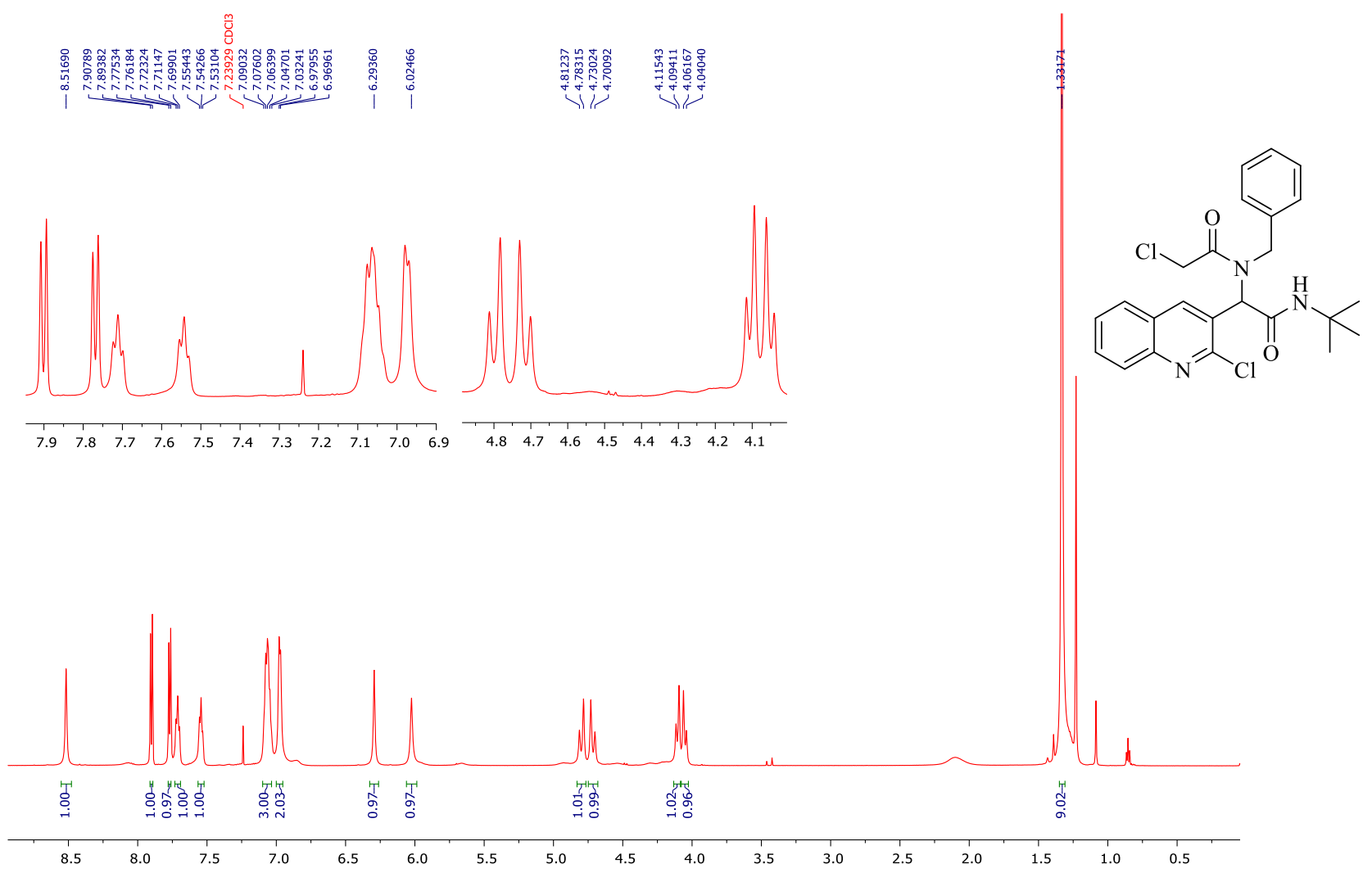

Figure S5: ${ }^{1} \mathrm{H}-\mathrm{NMR}$ of compound $\mathbf{5 b}\left(600 \mathrm{MHz}, \mathrm{CDCl}_{3}\right)$
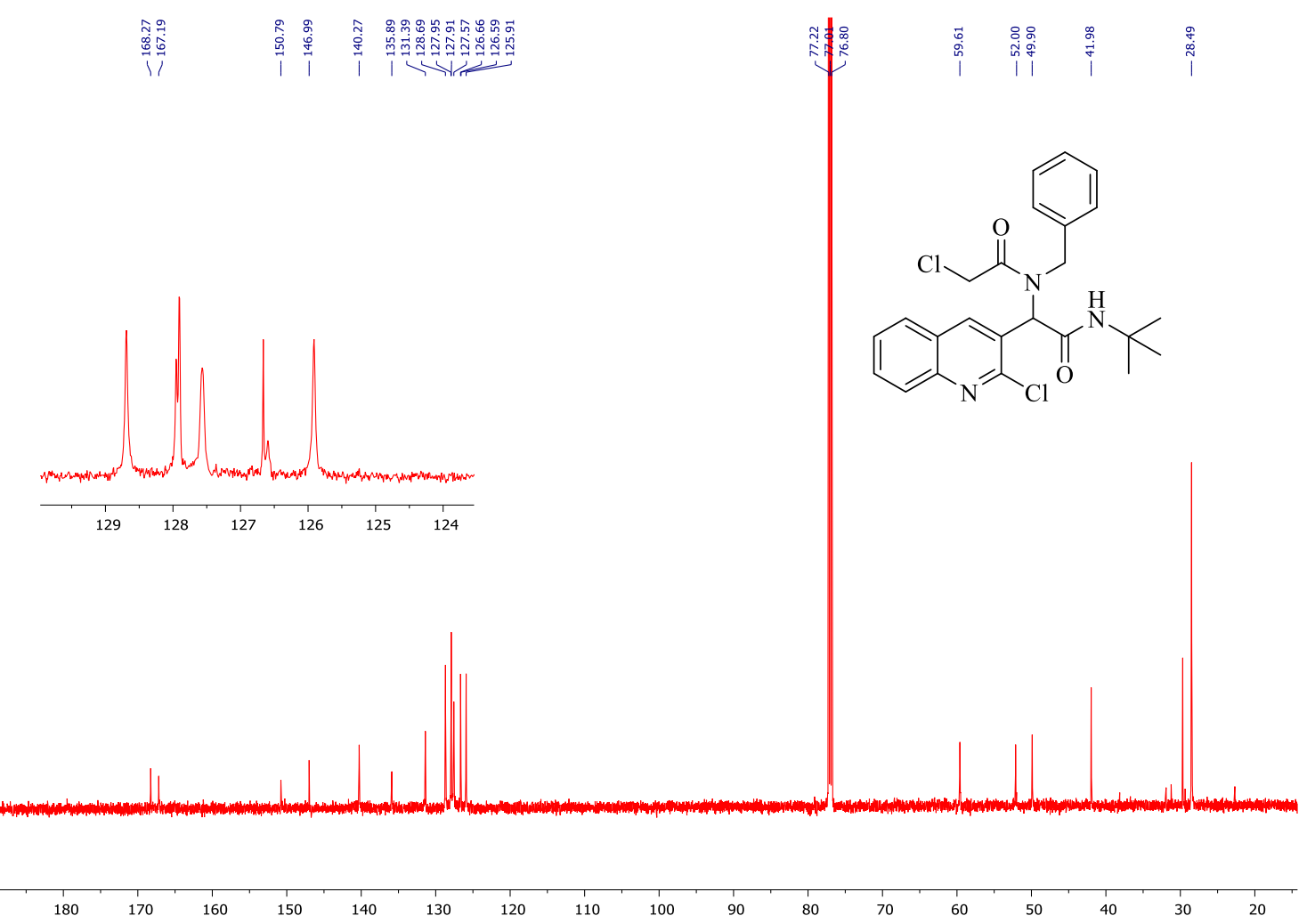

Figure S6: ${ }^{13} \mathrm{C}-\mathrm{NMR}$ of compound $\mathbf{5 b}\left(150 \mathrm{MHz}, \mathrm{CDCl}_{3}\right)$ 


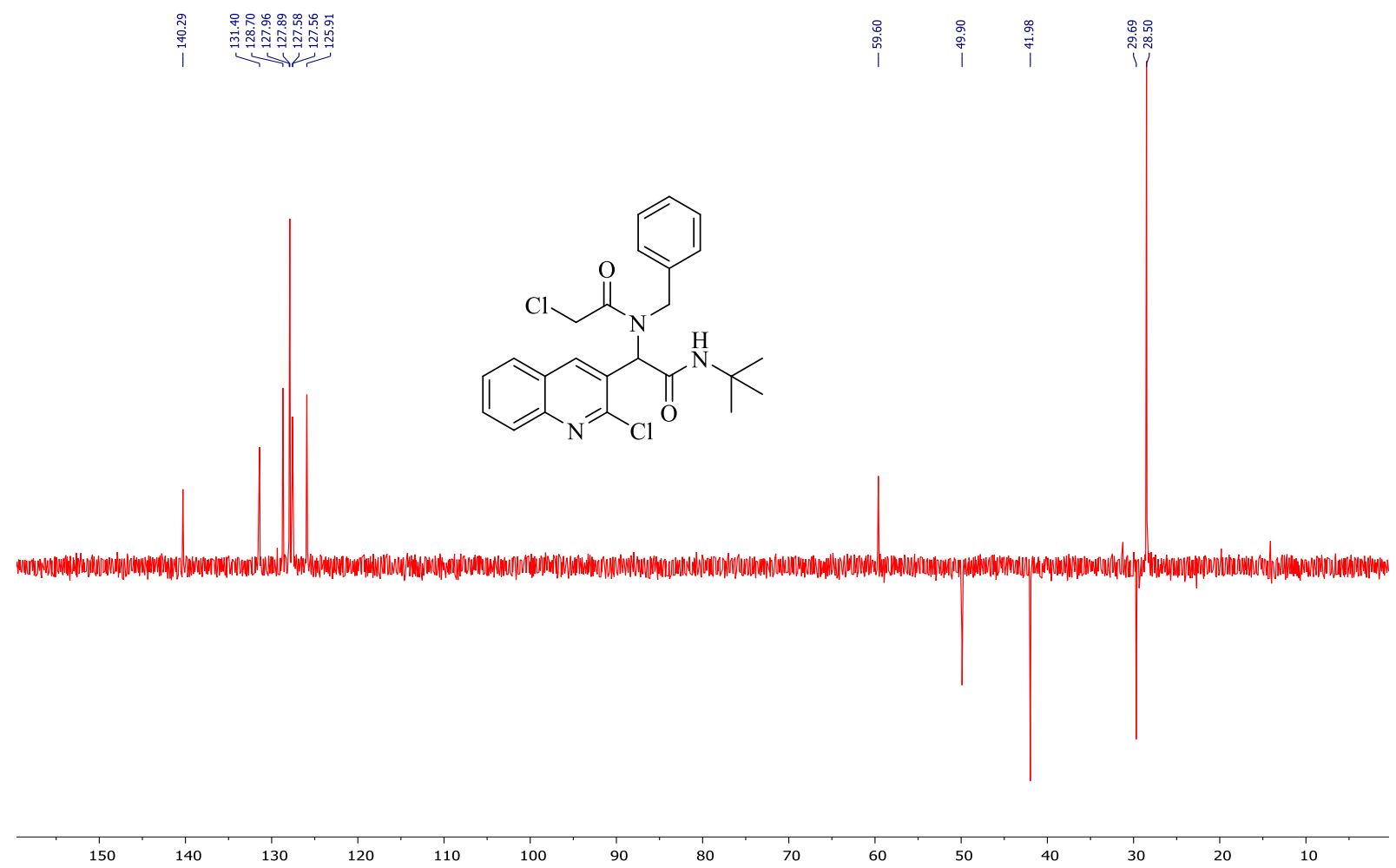

Figure S7: ${ }^{13} \mathrm{C}-\mathrm{NMR}$ (DEPT 135) of compound $\mathbf{5 b}\left(150 \mathrm{MHz}, \mathrm{CDCl}_{3}\right)$

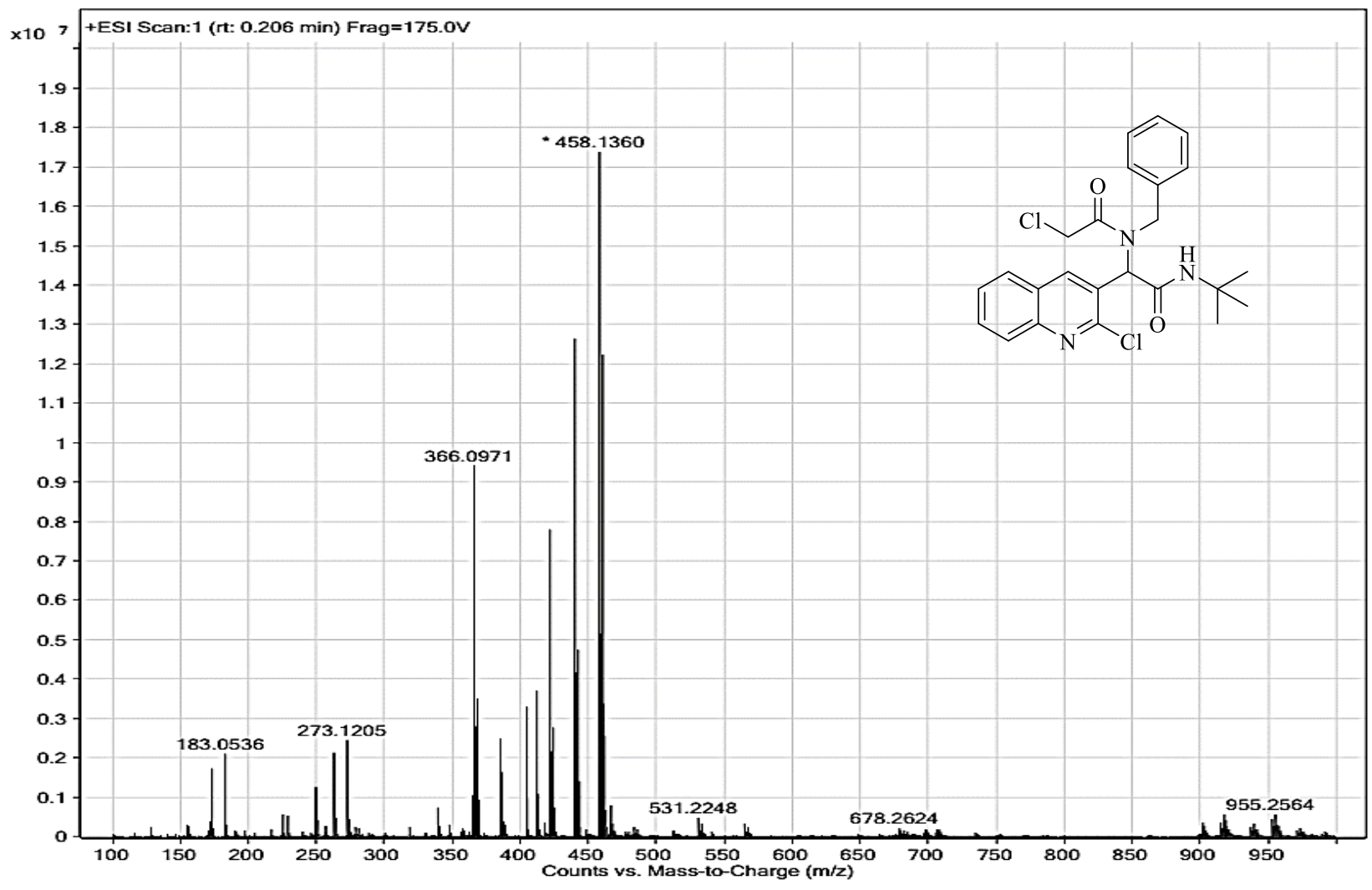

Figure S8: HRMS-ESI of $\mathbf{5 b}$ with formula $\mathrm{C}_{24} \mathrm{H}_{25} \mathrm{Cl}_{2} \mathrm{~N}_{3} \mathrm{O}_{2}$ and $[\mathrm{M}+\mathrm{H}]^{+} 458.1369$ 


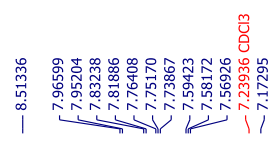

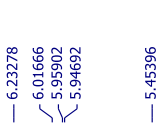
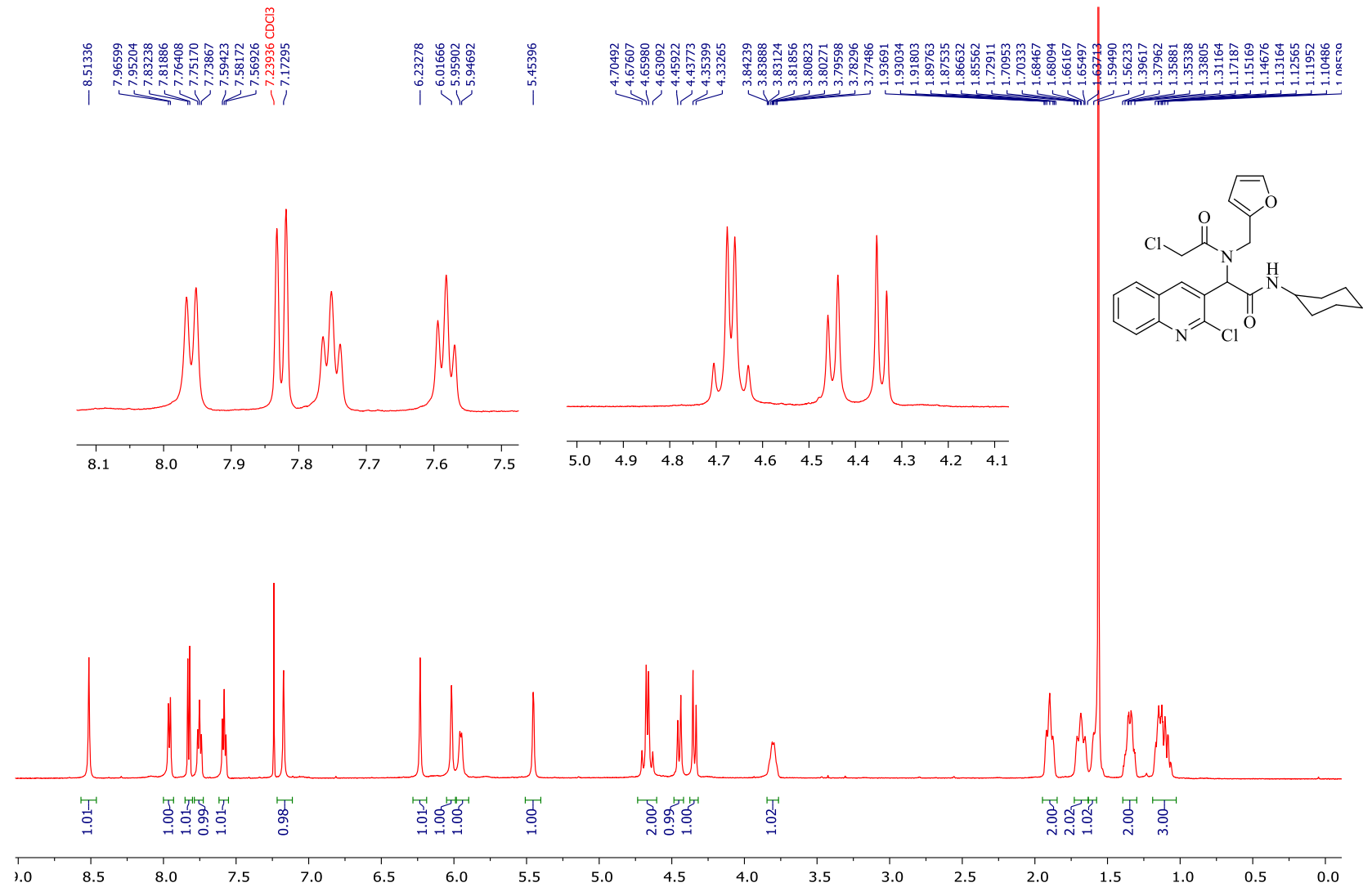

Figure S9: ${ }^{1} \mathrm{H}-\mathrm{NMR}$ of compound $\mathbf{5 c}\left(600 \mathrm{MHz}, \mathrm{CDCl}_{3}\right)$

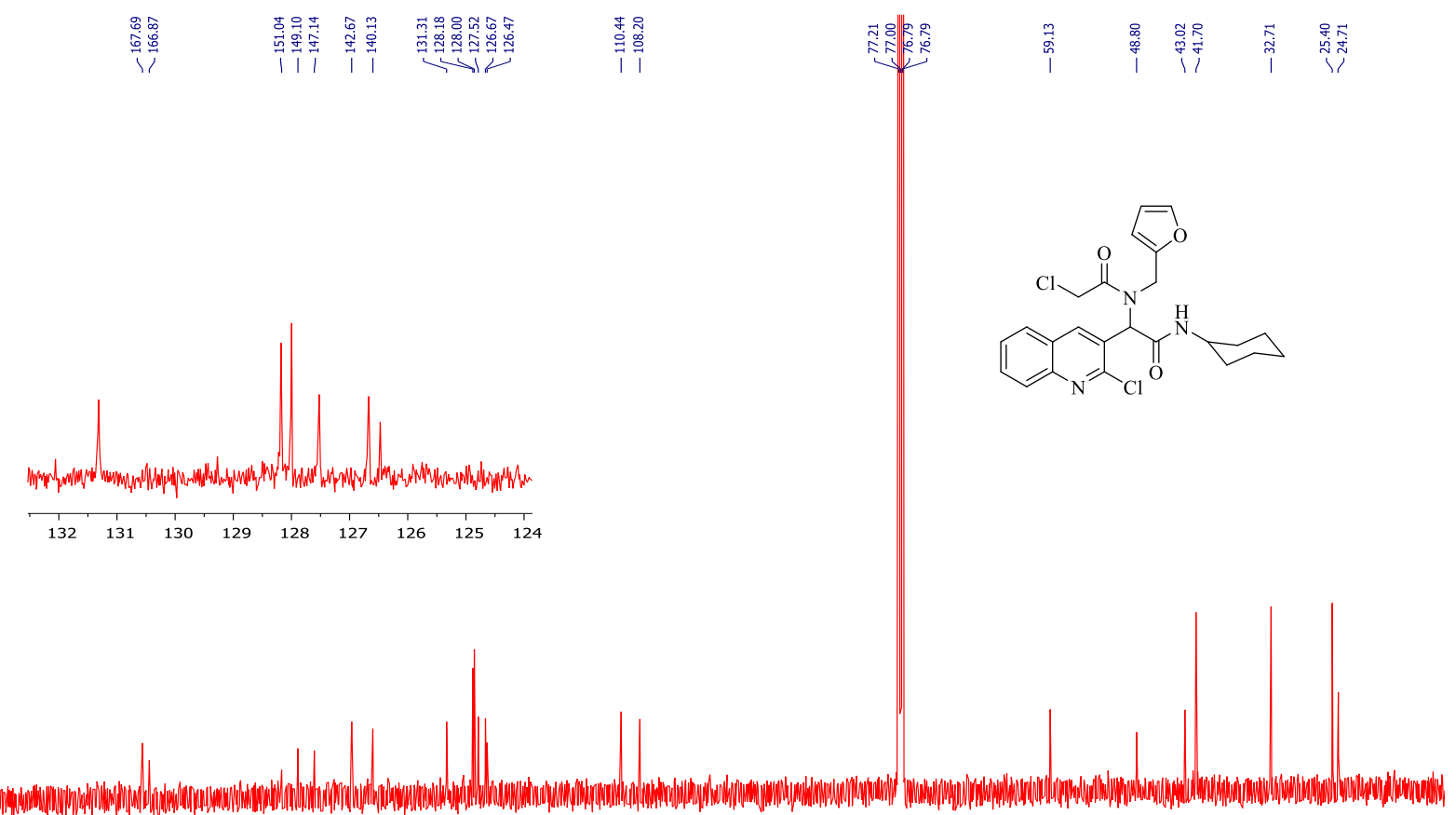

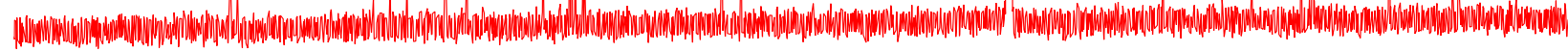

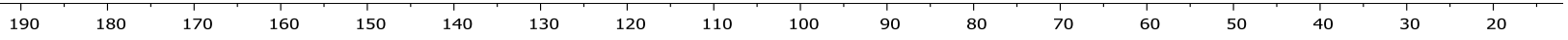

Figure S10: ${ }^{13} \mathrm{C}-\mathrm{NMR}$ of compound $\mathbf{5 c}\left(150 \mathrm{MHz}, \mathrm{CDCl}_{3}\right)$ 


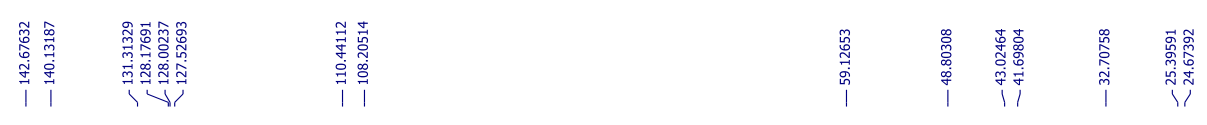

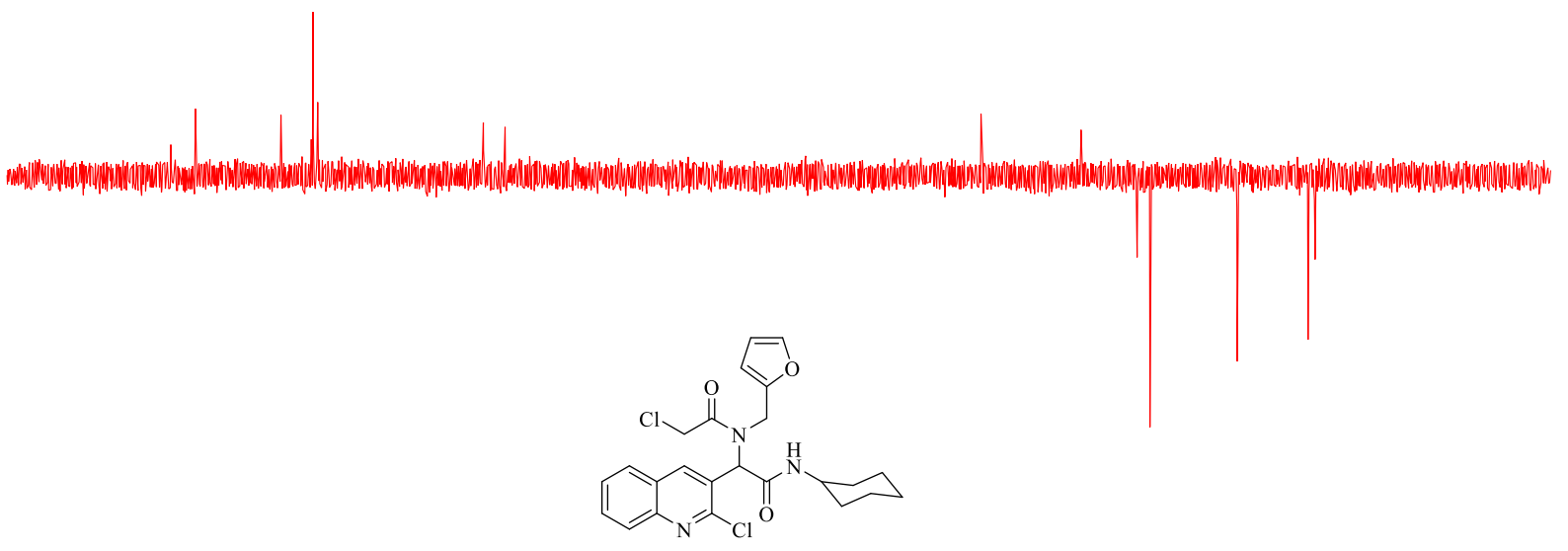

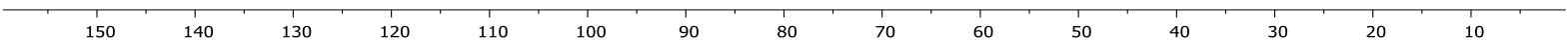

Figure S11: ${ }^{13} \mathrm{C}-\mathrm{NMR}$ (DEPT 135) of compound 5c $\left(150 \mathrm{MHz}, \mathrm{CDCl}_{3}\right)$

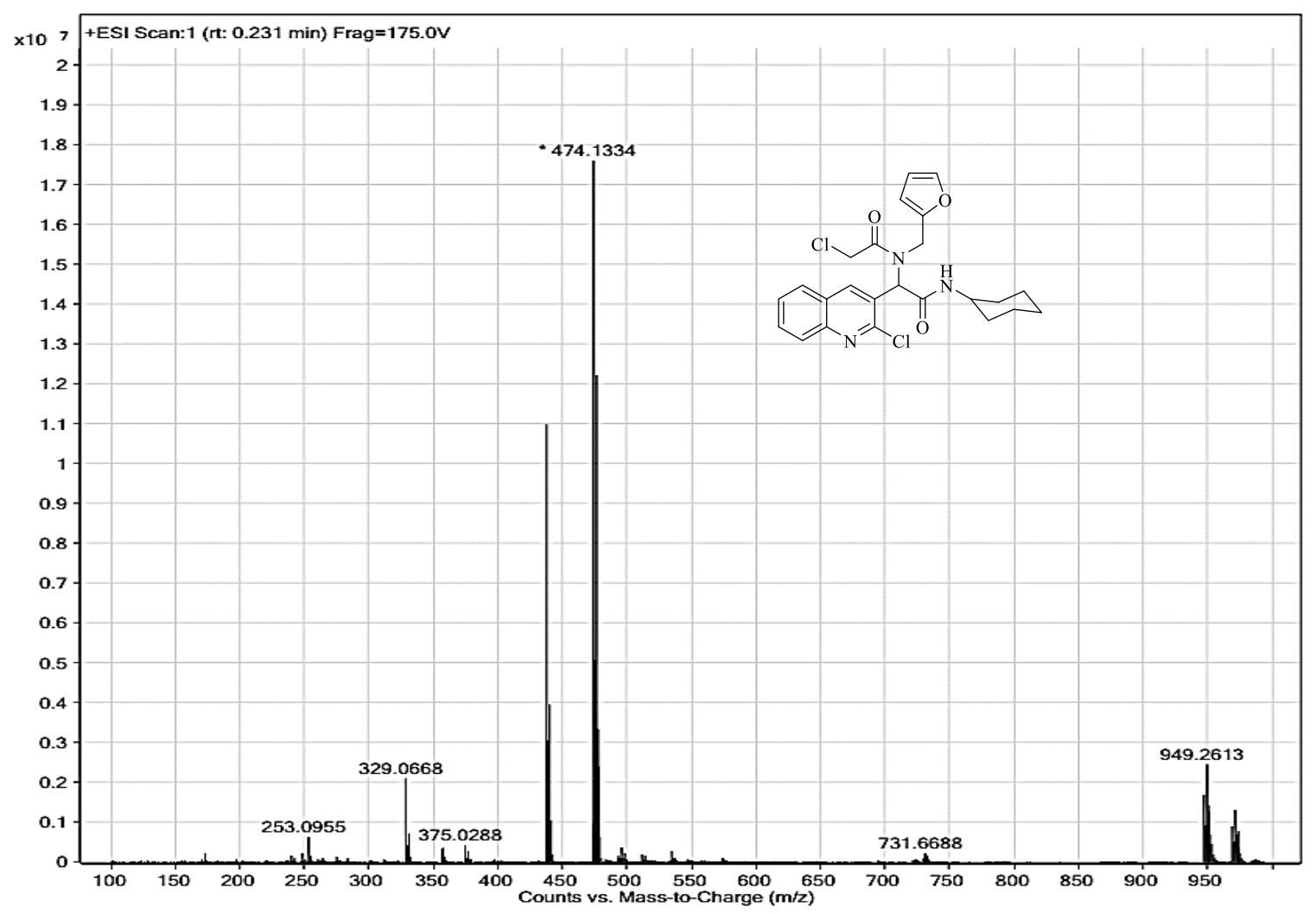

Figure S12: HRMS-ESI of 5c with formula $\mathrm{C}_{24} \mathrm{H}_{25} \mathrm{Cl}_{2} \mathrm{~N}_{3} \mathrm{O}_{3}$ and $[\mathrm{M}+\mathrm{H}]^{+} 474.1341$ 


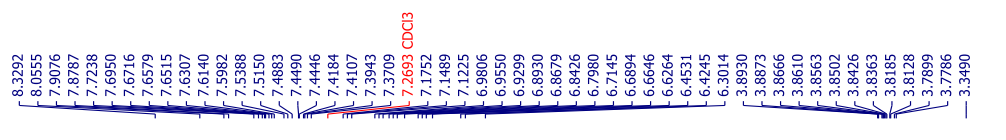

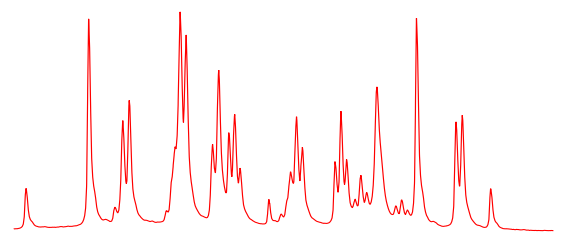

$\begin{array}{llllllllllllll}8.2 & 8.0 & 7.8 & 7.6 & 7.4 & 7.2 & 7.0 & 6.8 & 6.6 & 6.4 & 6.2\end{array}$

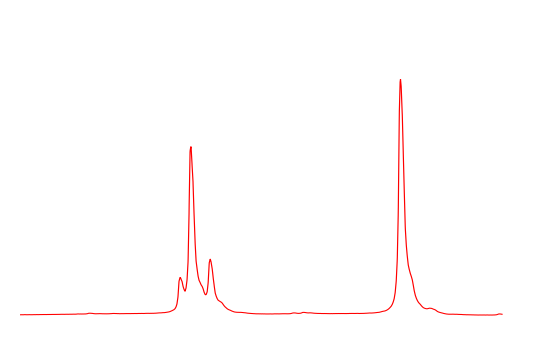

$\begin{array}{lllllllllllllllll}4.2 & 4.1 & 4.0 & 3.9 & 3.8 & 3.7 & 3.6 & 3.5 & 3.4 & 3.3 & 3.2 & 3.1\end{array}$

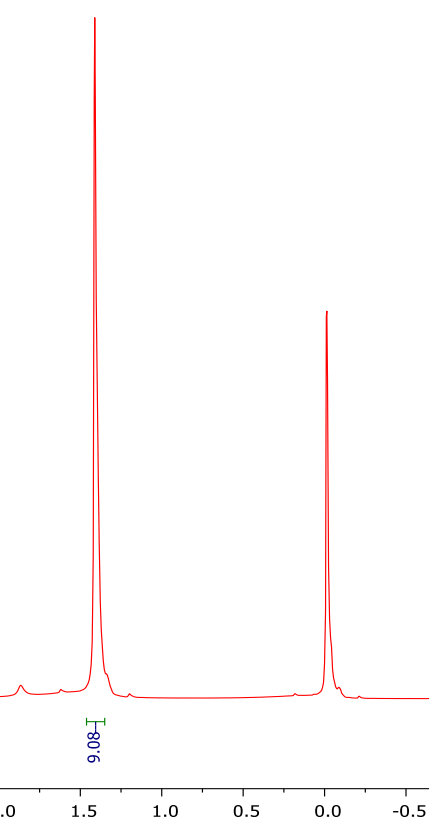

Figure S13: ${ }^{1} \mathrm{H}-\mathrm{NMR}$ of compound $\mathbf{5 d}\left(300 \mathrm{MHz}, \mathrm{CDCl}_{3}\right)$
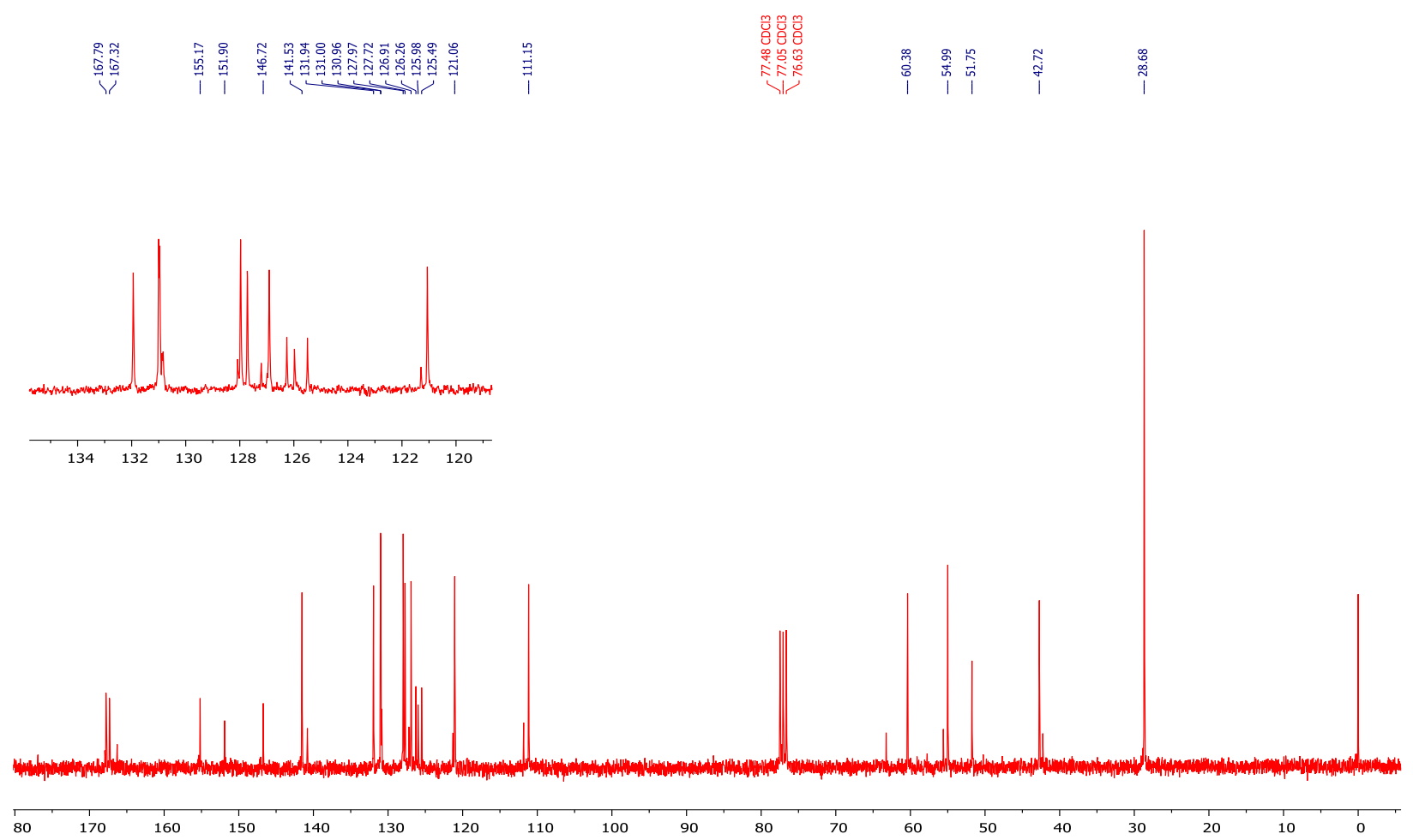

Figure S14: ${ }^{13} \mathrm{C}-\mathrm{NMR}$ of compound $\mathbf{5 d}\left(75 \mathrm{MHz}, \mathrm{CDCl}_{3}\right)$ 

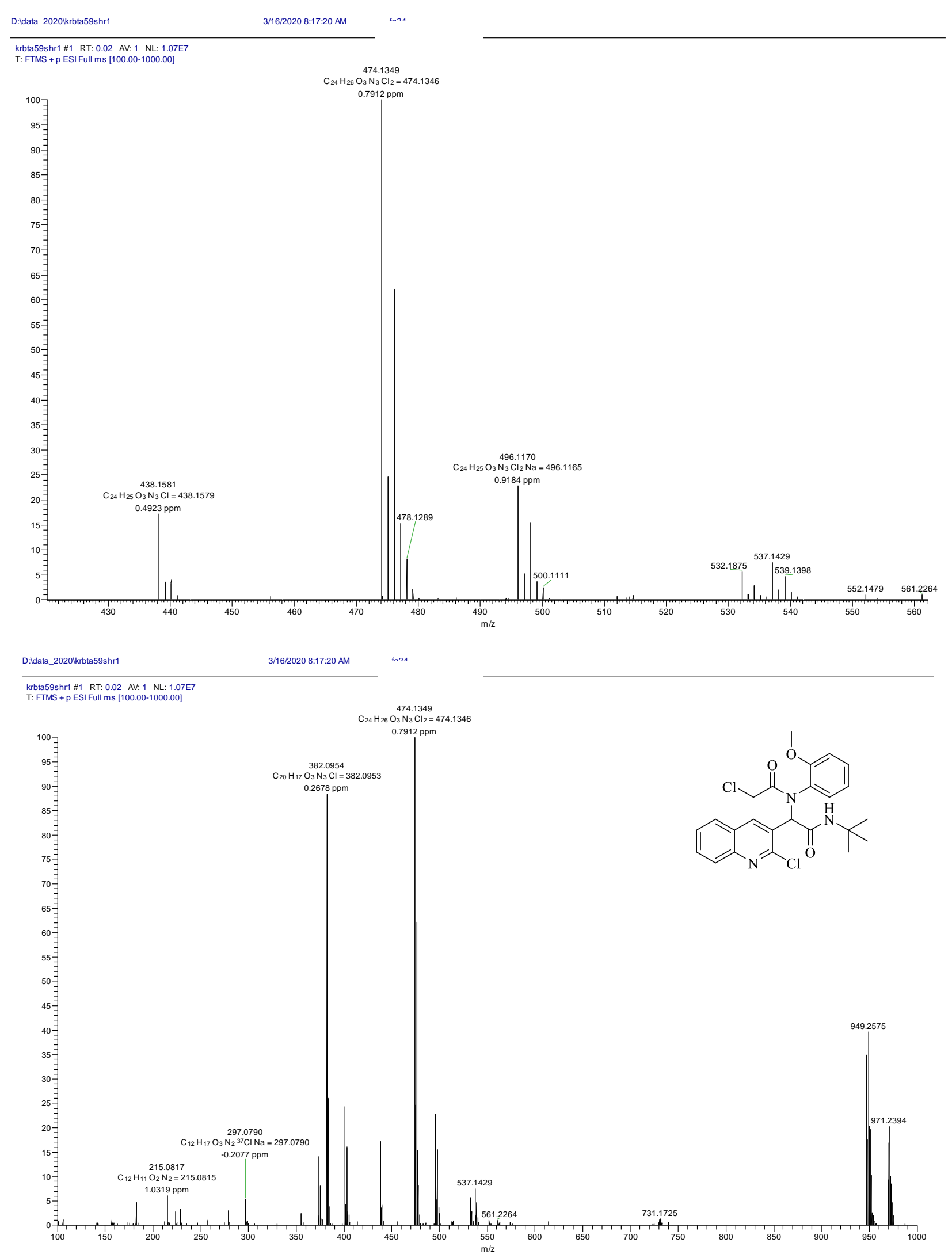

Figure S15: HRMS-ESI of $\mathbf{5 d}$ with formula $\mathrm{C}_{24} \mathrm{H}_{25} \mathrm{Cl}_{2} \mathrm{~N}_{3} \mathrm{O}_{3}$ and $[\mathrm{M}+\mathrm{H}]^{+} 474.1346$ 

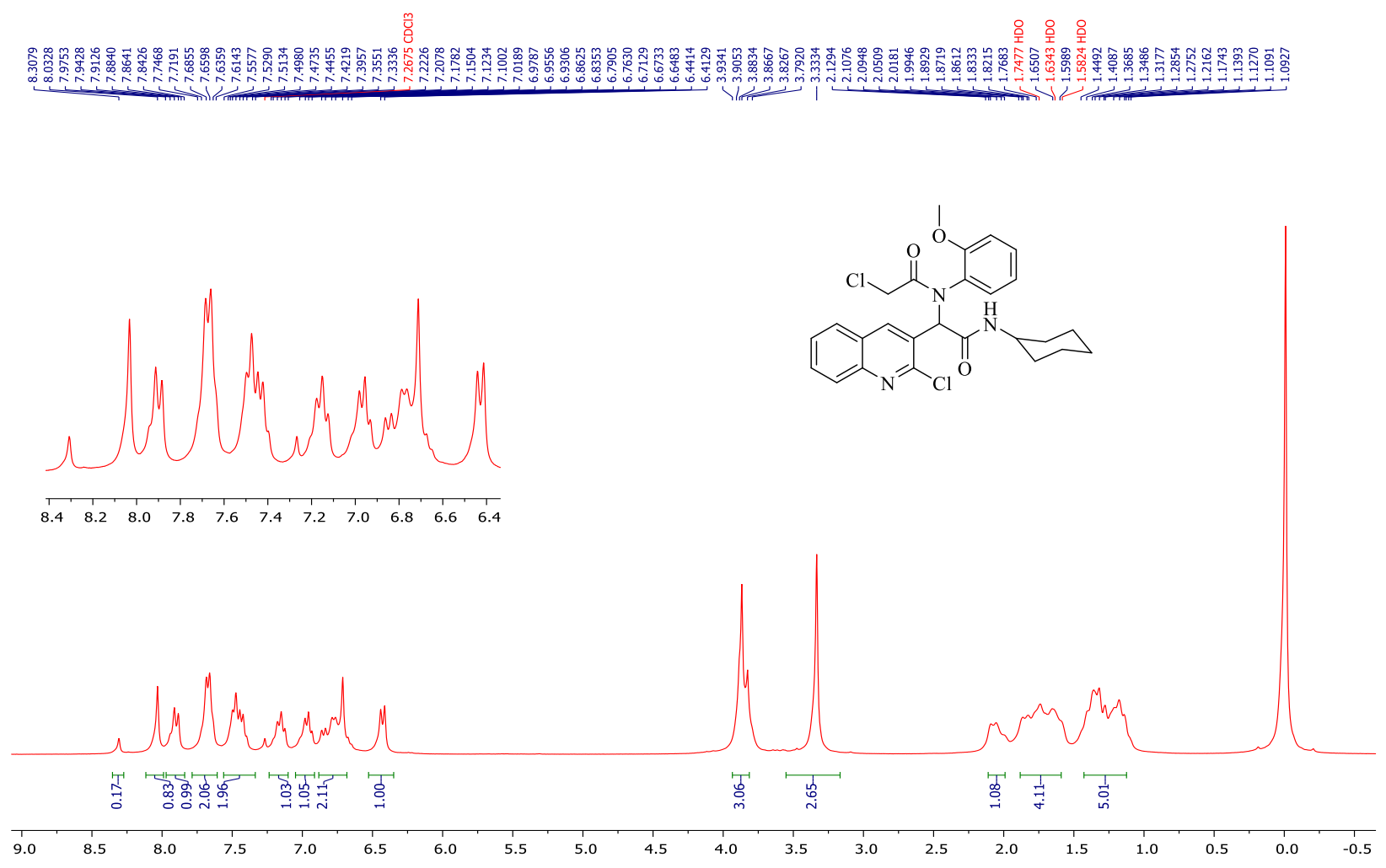

Figure S16: ${ }^{1} \mathrm{H}-\mathrm{NMR}$ of compound 5 e $\left(300 \mathrm{MHz}, \mathrm{CDCl}_{3}\right)$
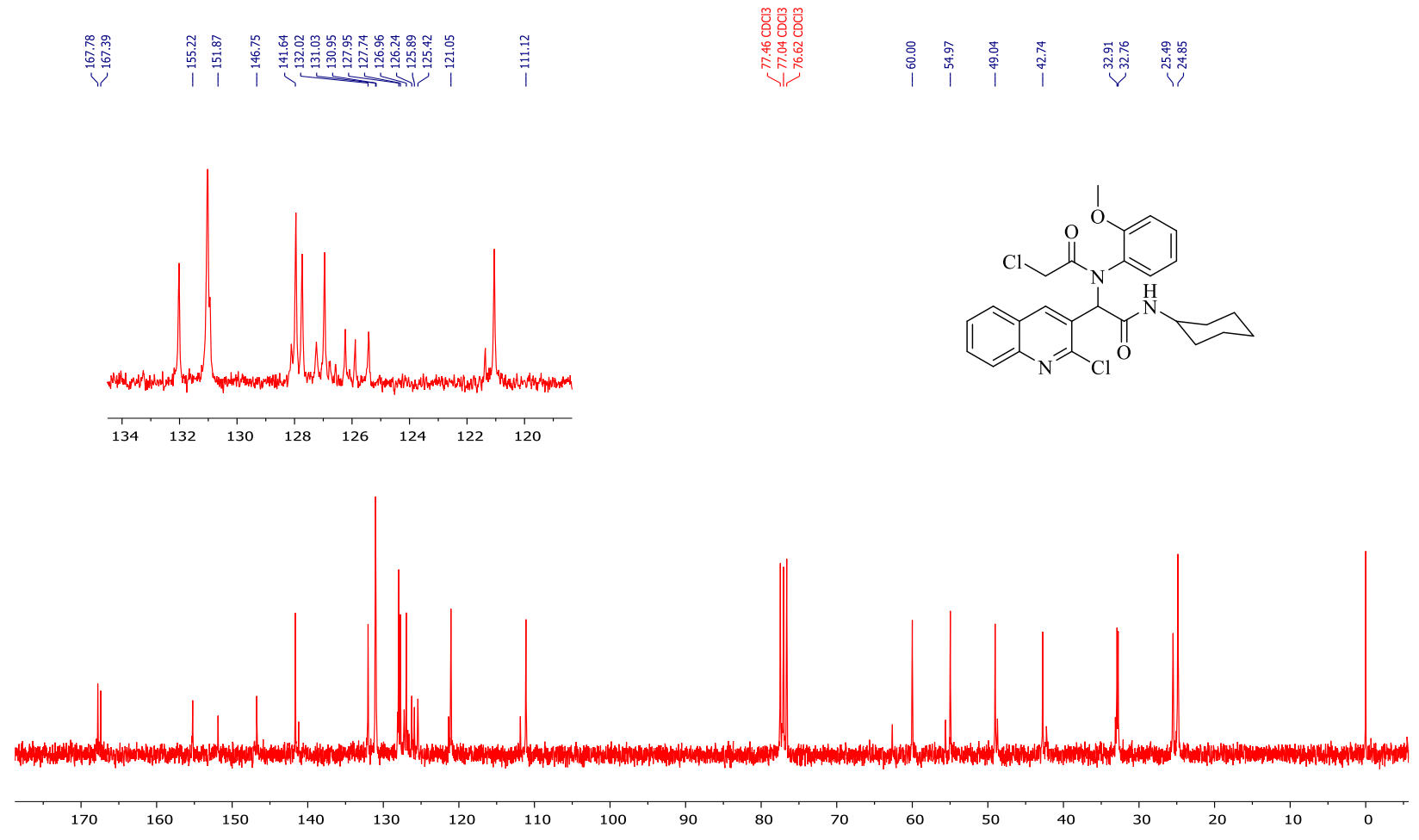

Figure S17: ${ }^{13} \mathrm{C}-\mathrm{NMR}$ of compound $5 \mathbf{e}\left(75 \mathrm{MHz}, \mathrm{CDCl}_{3}\right)$ 


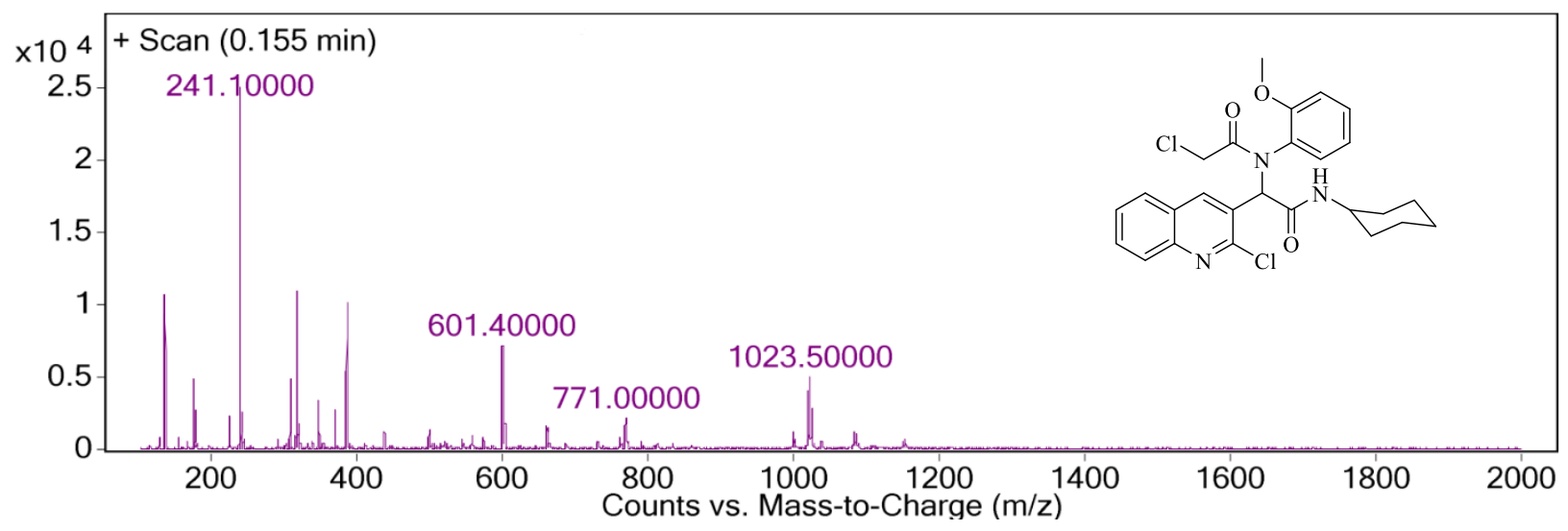

Figure S18: ESI-MS (+) of compound 5e with formula $\mathrm{C}_{52} \mathrm{H}_{54} \mathrm{Cl}_{4} \mathrm{~N}_{6} \mathrm{NaO}_{6}$ and $[2 \mathrm{M}+\mathrm{Na}]^{+} 1023.5$. 


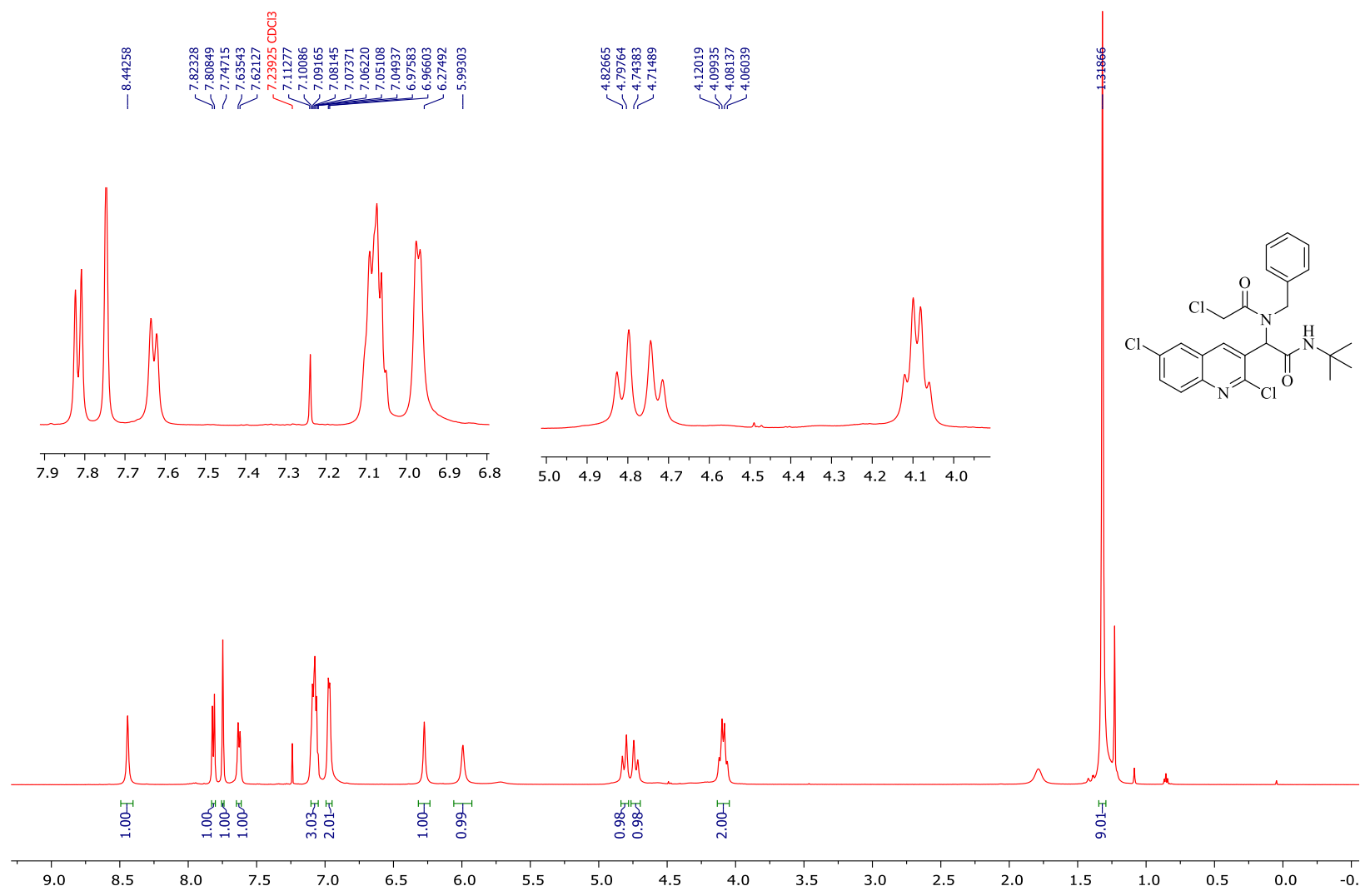

Figure S19: ${ }^{1} \mathrm{H}-\mathrm{NMR}$ of compound $\mathbf{5 f}\left(600 \mathrm{MHz}, \mathrm{CDCl}_{3}\right)$

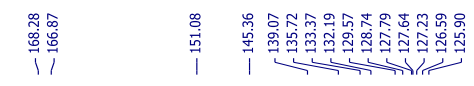

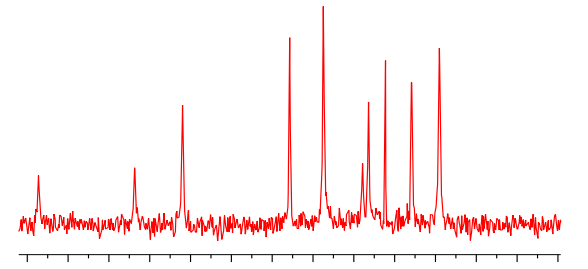

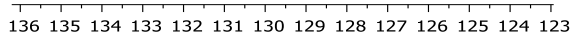
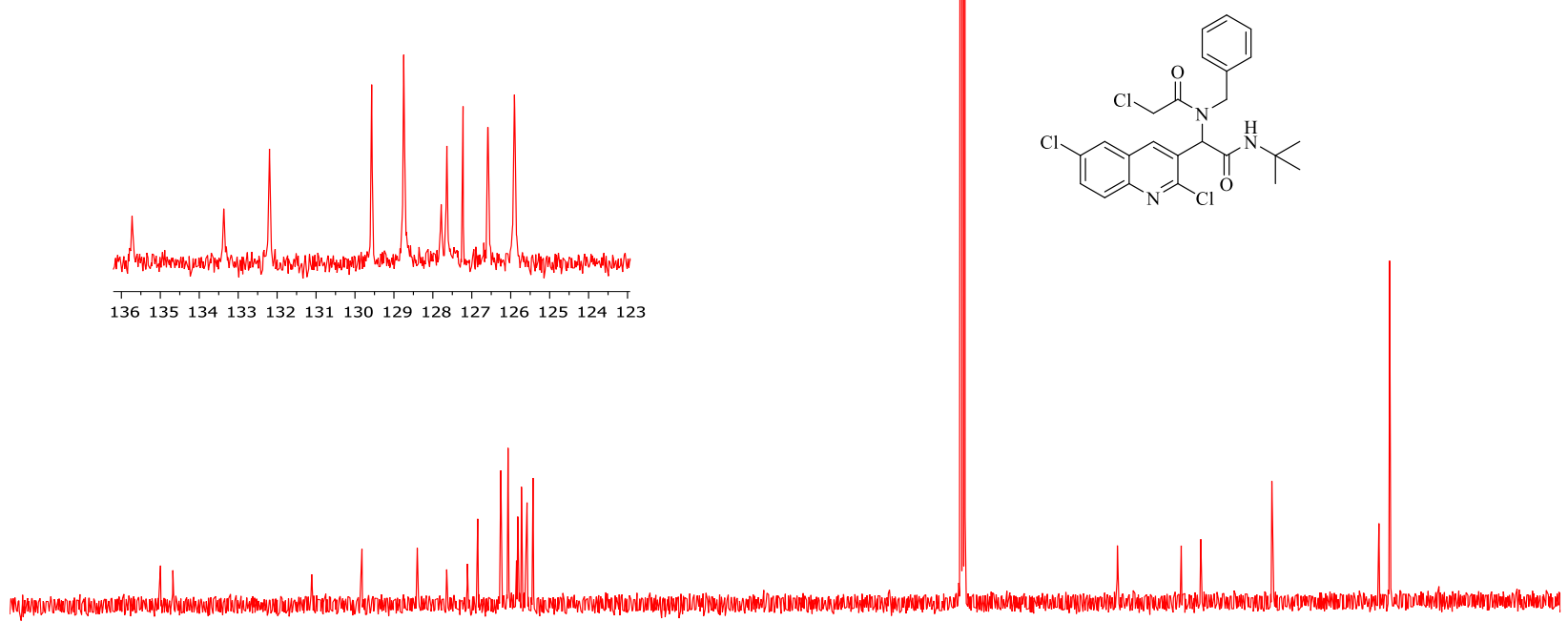

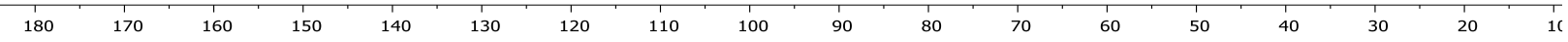

Figure S20: ${ }^{13} \mathrm{C}-\mathrm{NMR}$ of compound $\mathbf{5 f}\left(150 \mathrm{MHz}, \mathrm{CDCl}_{3}\right)$ 


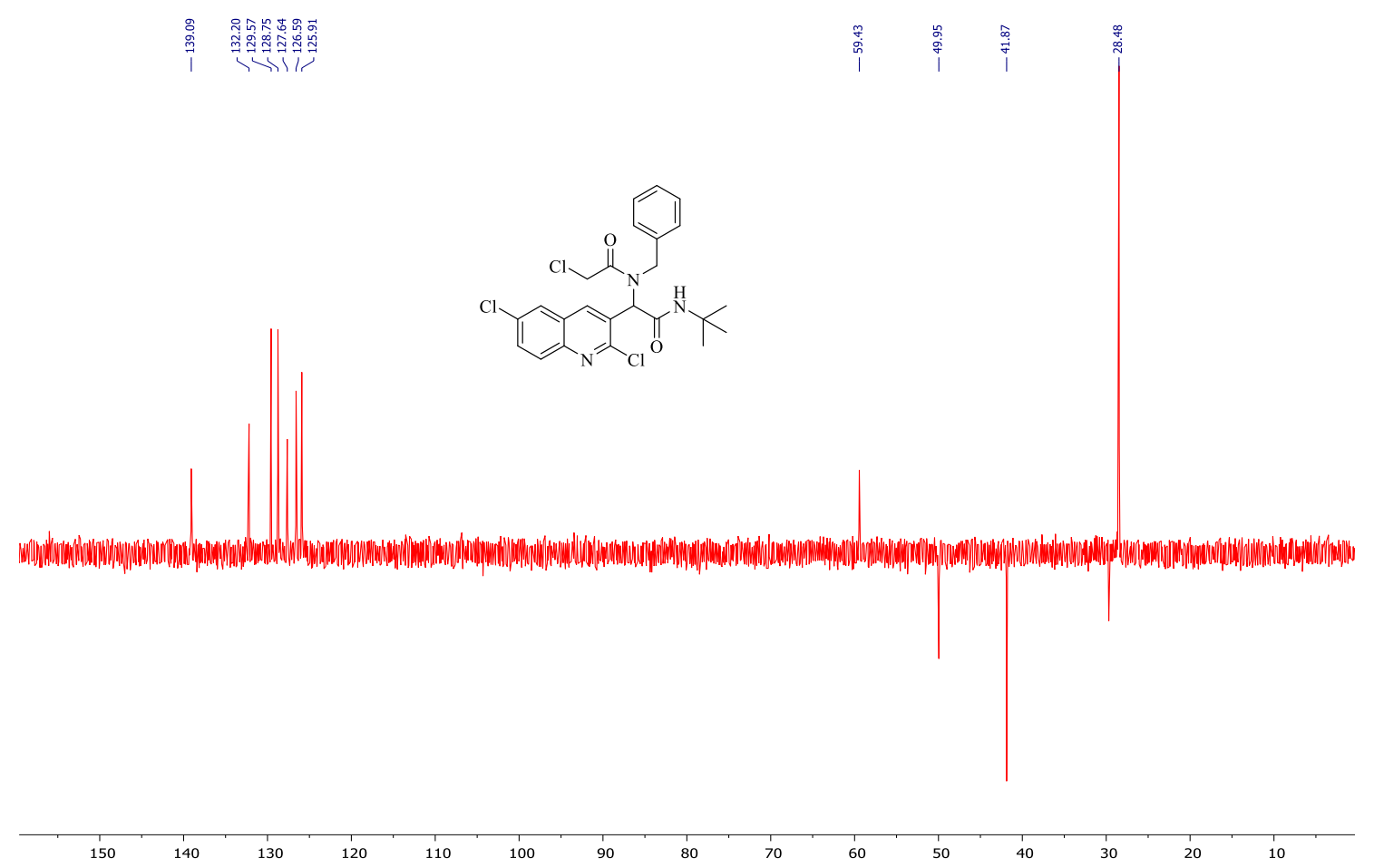

Figure S21: ${ }^{13} \mathrm{C}-\mathrm{NMR}$ (DEPT 135) of compound $\mathbf{5 f}\left(150 \mathrm{MHz}, \mathrm{CDCl}_{3}\right)$

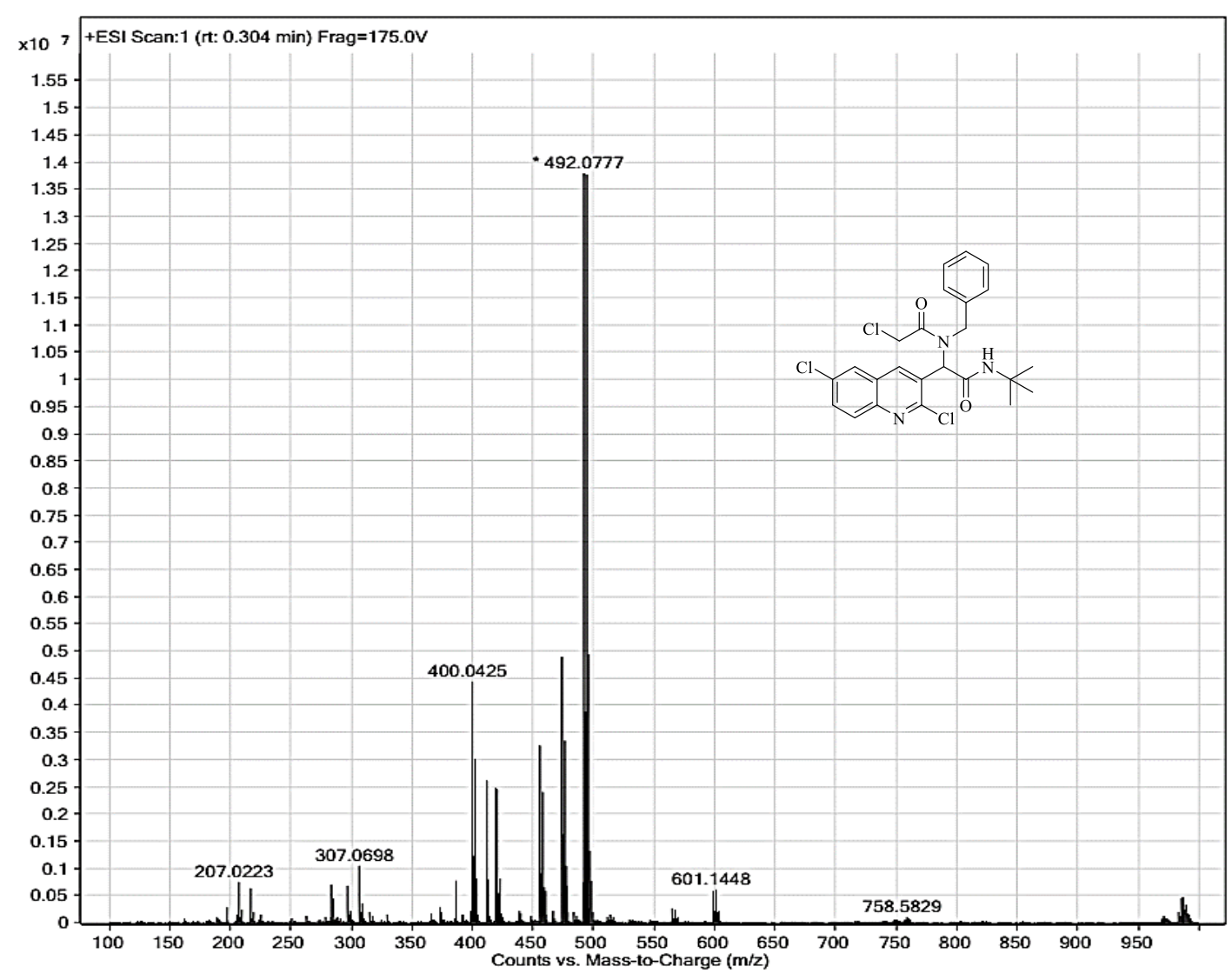

Figure S22: HRMS-ESI of $\mathbf{5 f}$ with formula $\mathrm{C}_{24} \mathrm{H}_{24} \mathrm{Cl}_{3} \mathrm{~N}_{3} \mathrm{O}_{2}$ and $[\mathrm{M}+\mathrm{H}]^{+} 492.0786$ 


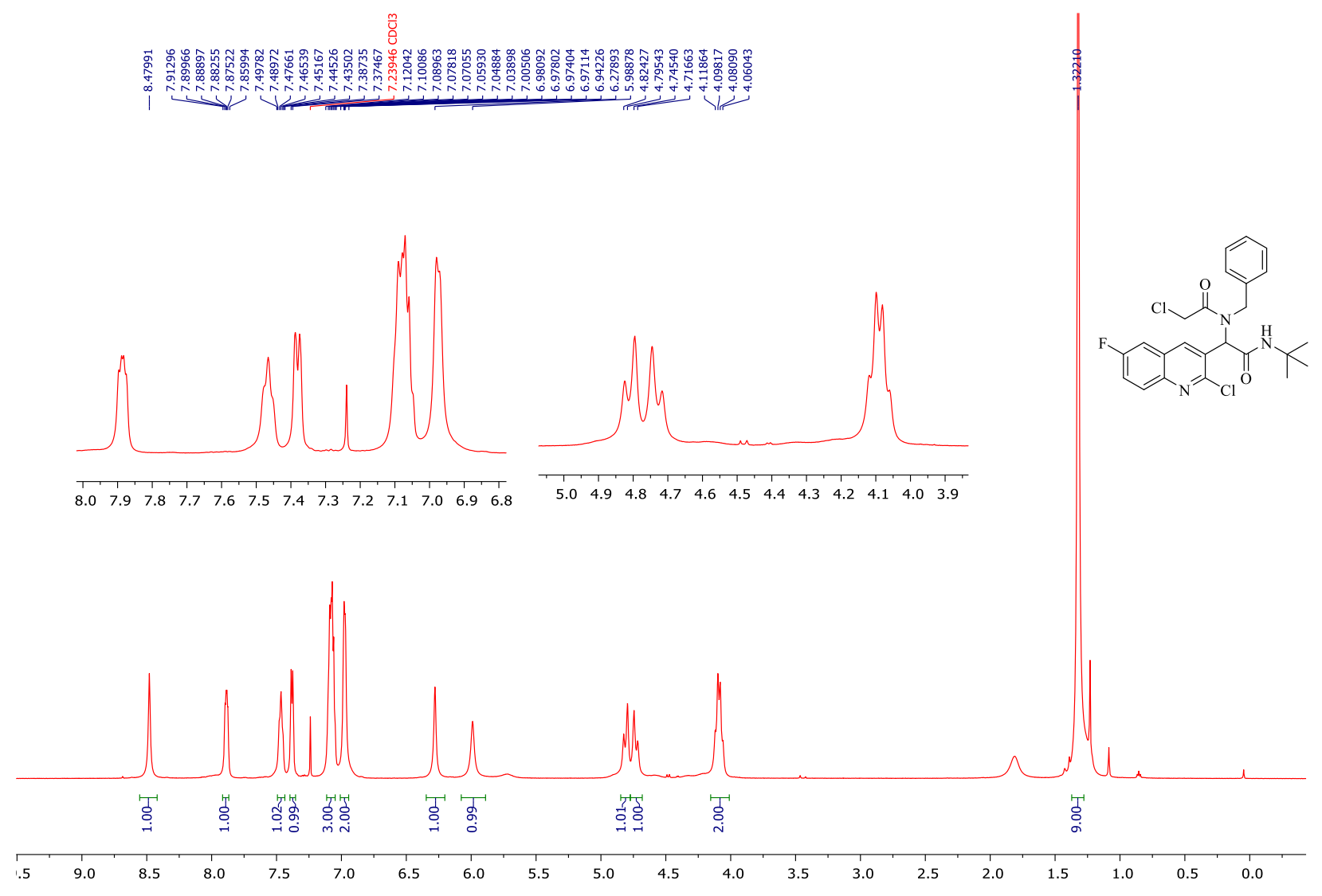

Figure S23: ${ }^{1} \mathrm{H}-\mathrm{NMR}$ of compound $\mathbf{5 g}\left(600 \mathrm{MHz}, \mathrm{CDCl}_{3}\right)$

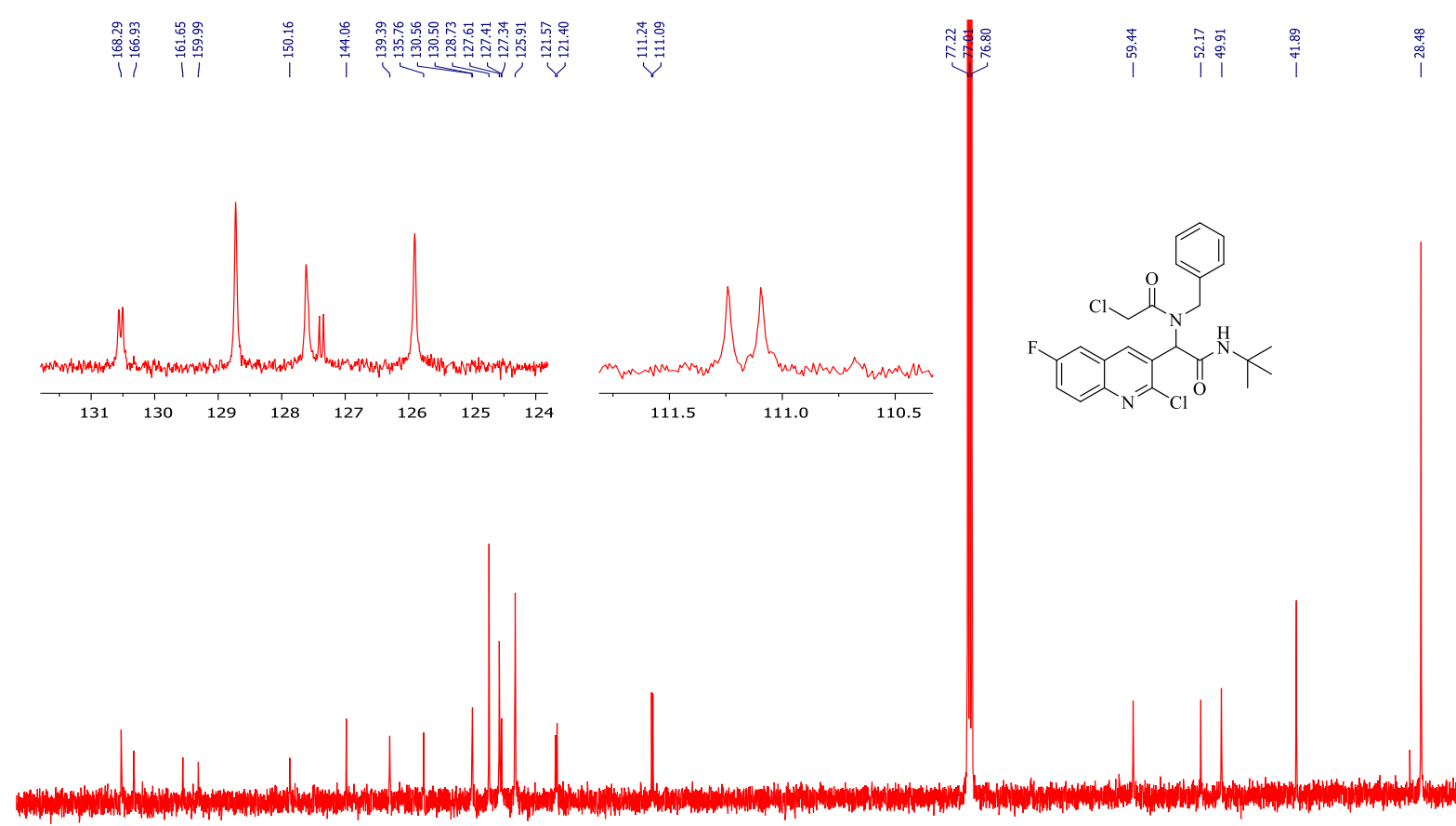



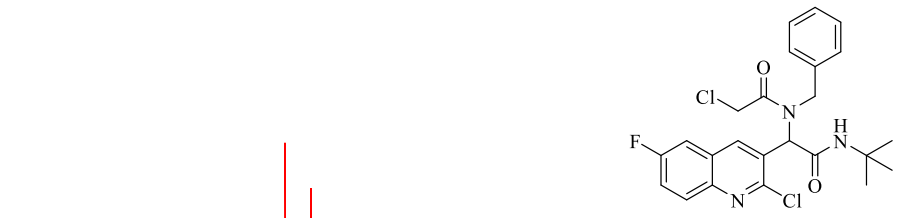

Figure S25: ${ }^{13} \mathrm{C}-\mathrm{NMR}$ (DEPT 135$)$ of compound $\mathbf{5 g}\left(150 \mathrm{MHz}, \mathrm{CDCl}_{3}\right)$

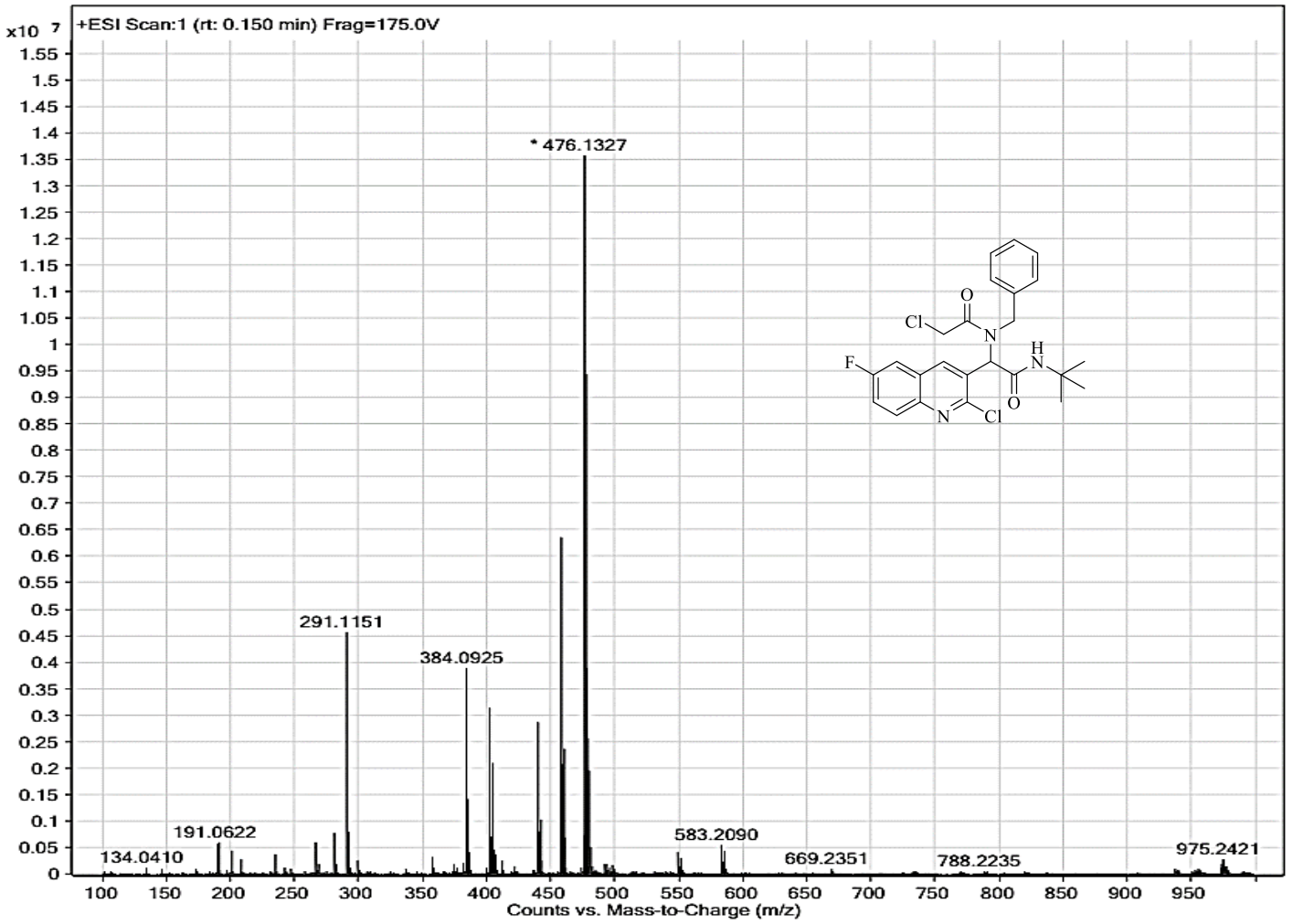

Figure S26: HRMS-ESI of $\mathbf{5 g}$ with formula $\mathrm{C}_{24} \mathrm{H}_{24} \mathrm{Cl}_{2} \mathrm{FN}_{3} \mathrm{O}_{2}$ and $[\mathrm{M}+\mathrm{H}]^{+} 476.1318$ 


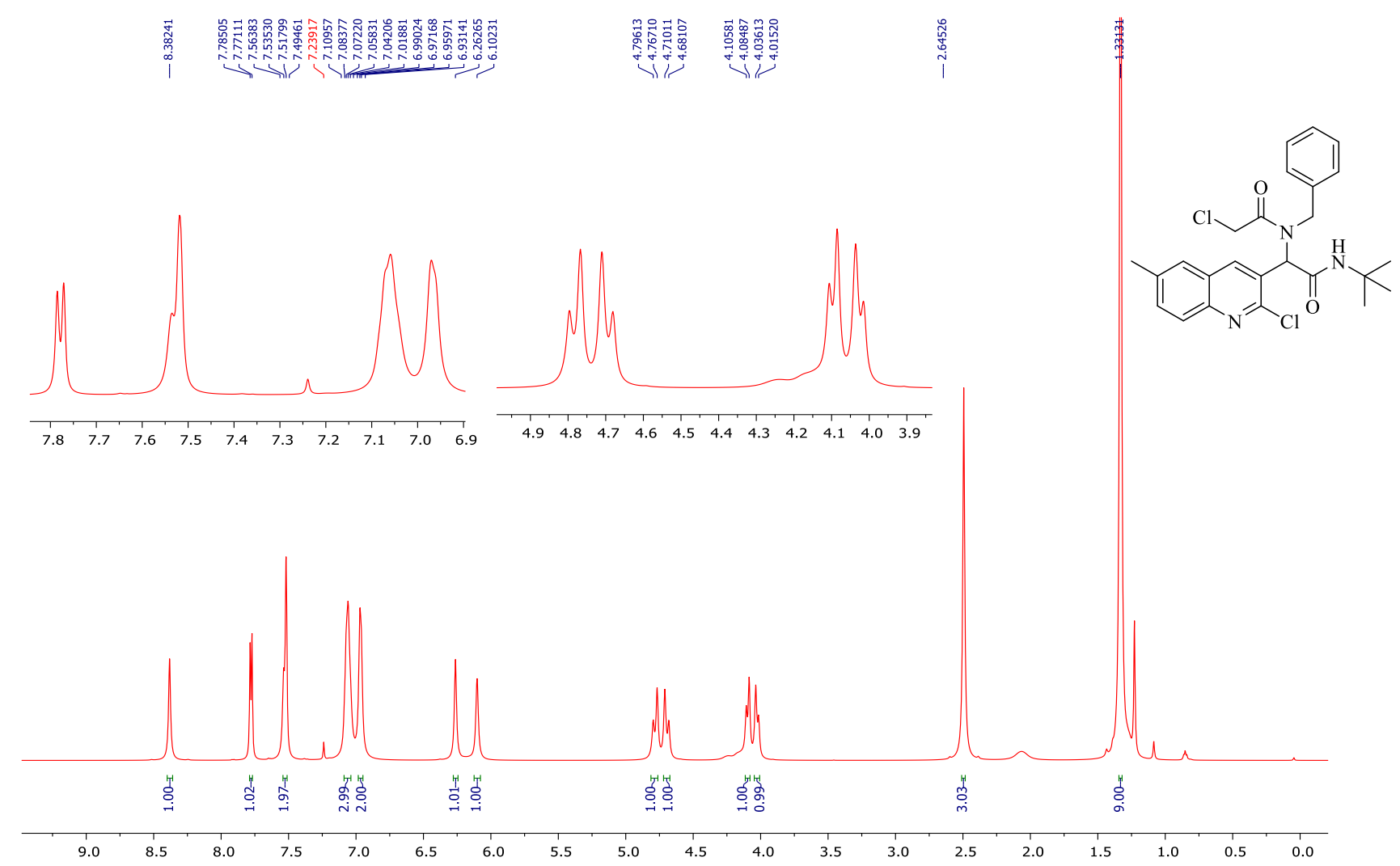

Figure S27: ${ }^{1} \mathrm{H}-\mathrm{NMR}$ of compound $\mathbf{5 h}\left(600 \mathrm{MHz}, \mathrm{CDCl}_{3}\right)$

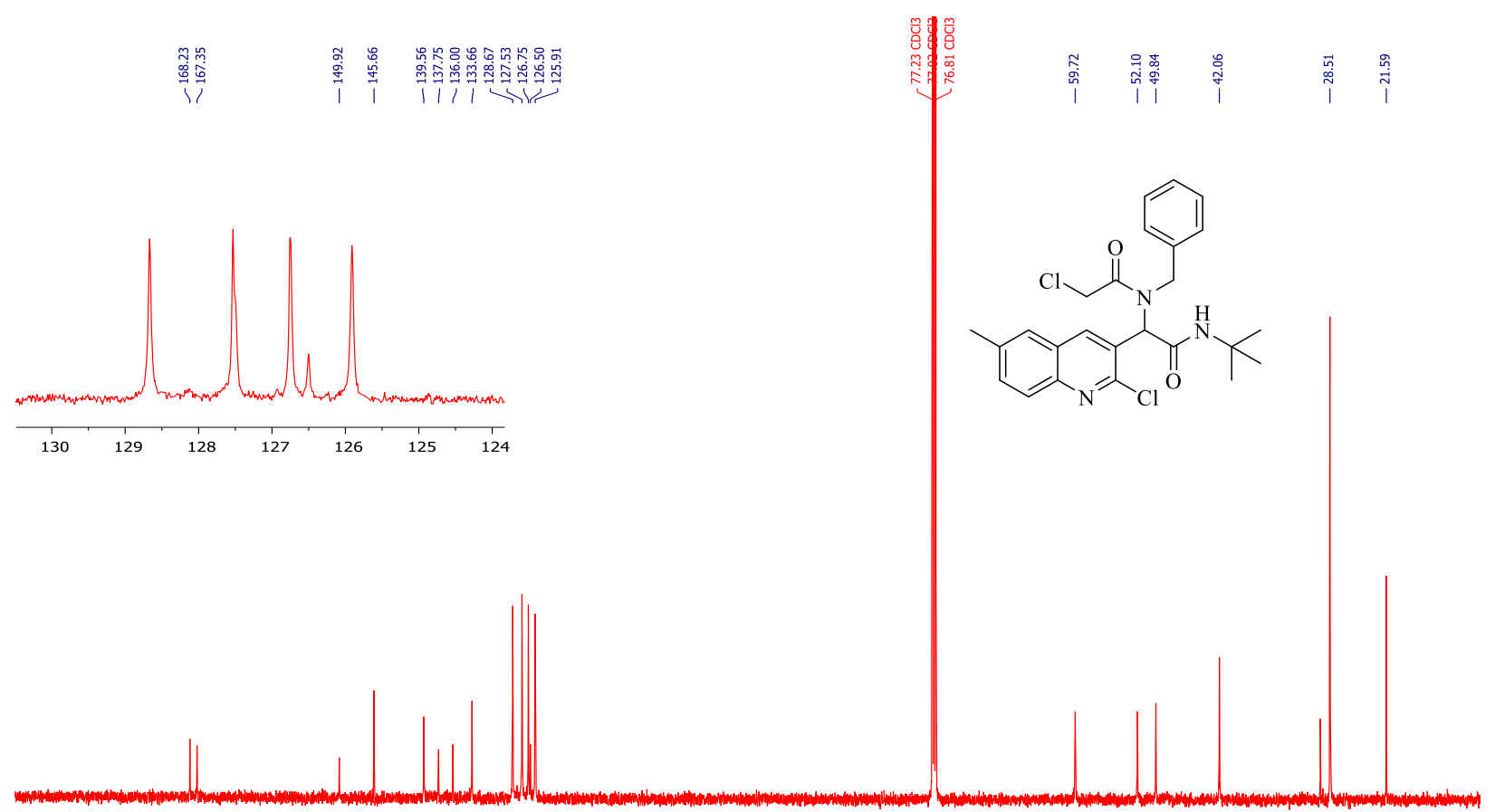

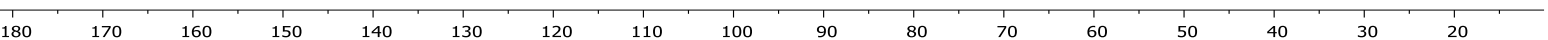

Figure S28: ${ }^{13} \mathrm{C}-\mathrm{NMR}$ of compound $\mathbf{5 h}\left(150 \mathrm{MHz}, \mathrm{CDCl}_{3}\right)$ 


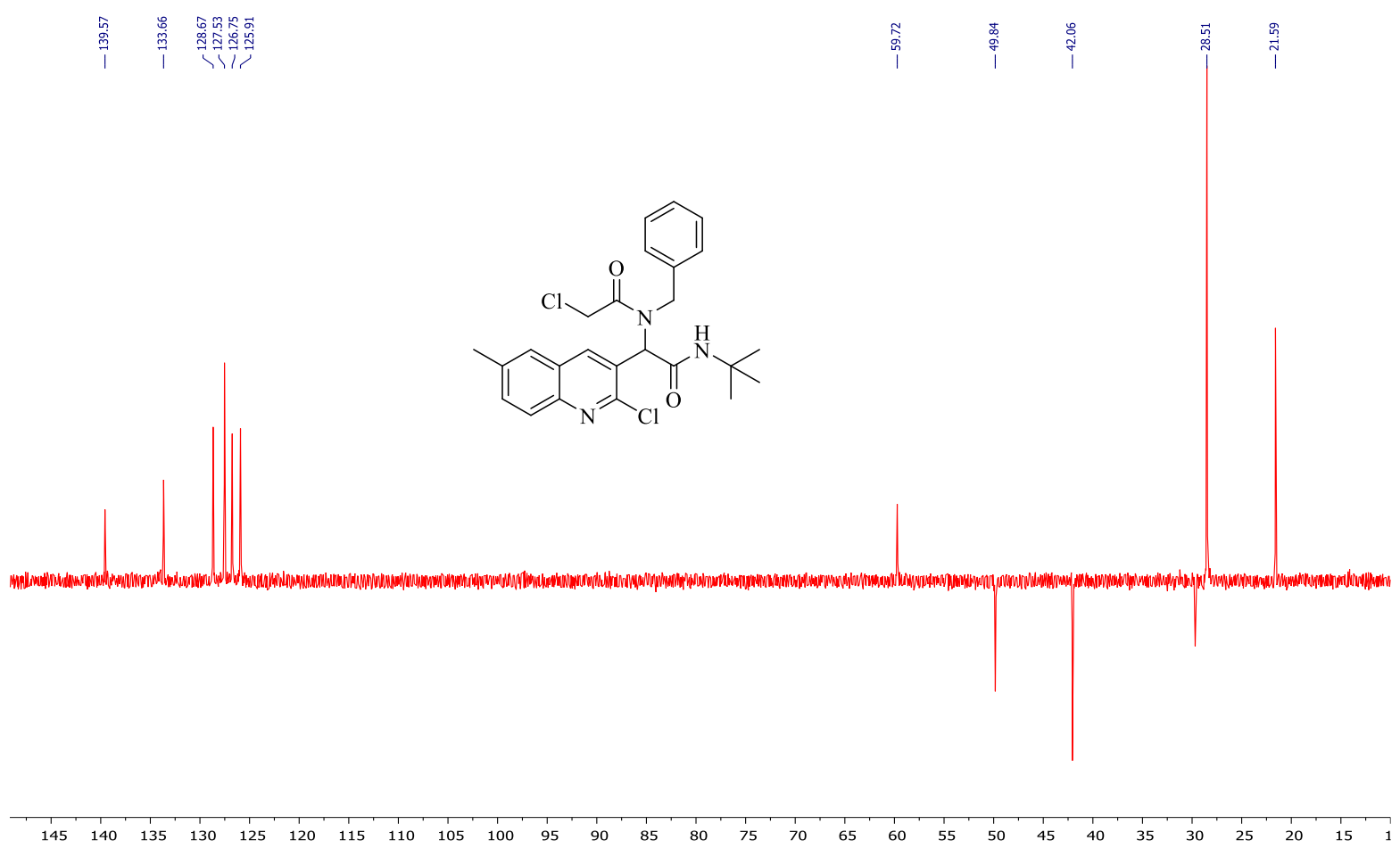

Figure S29: ${ }^{13} \mathrm{C}-\mathrm{NMR}$ (DEPT 135) of compound $\mathbf{5 h}\left(150 \mathrm{MHz}, \mathrm{CDCl}_{3}\right)$

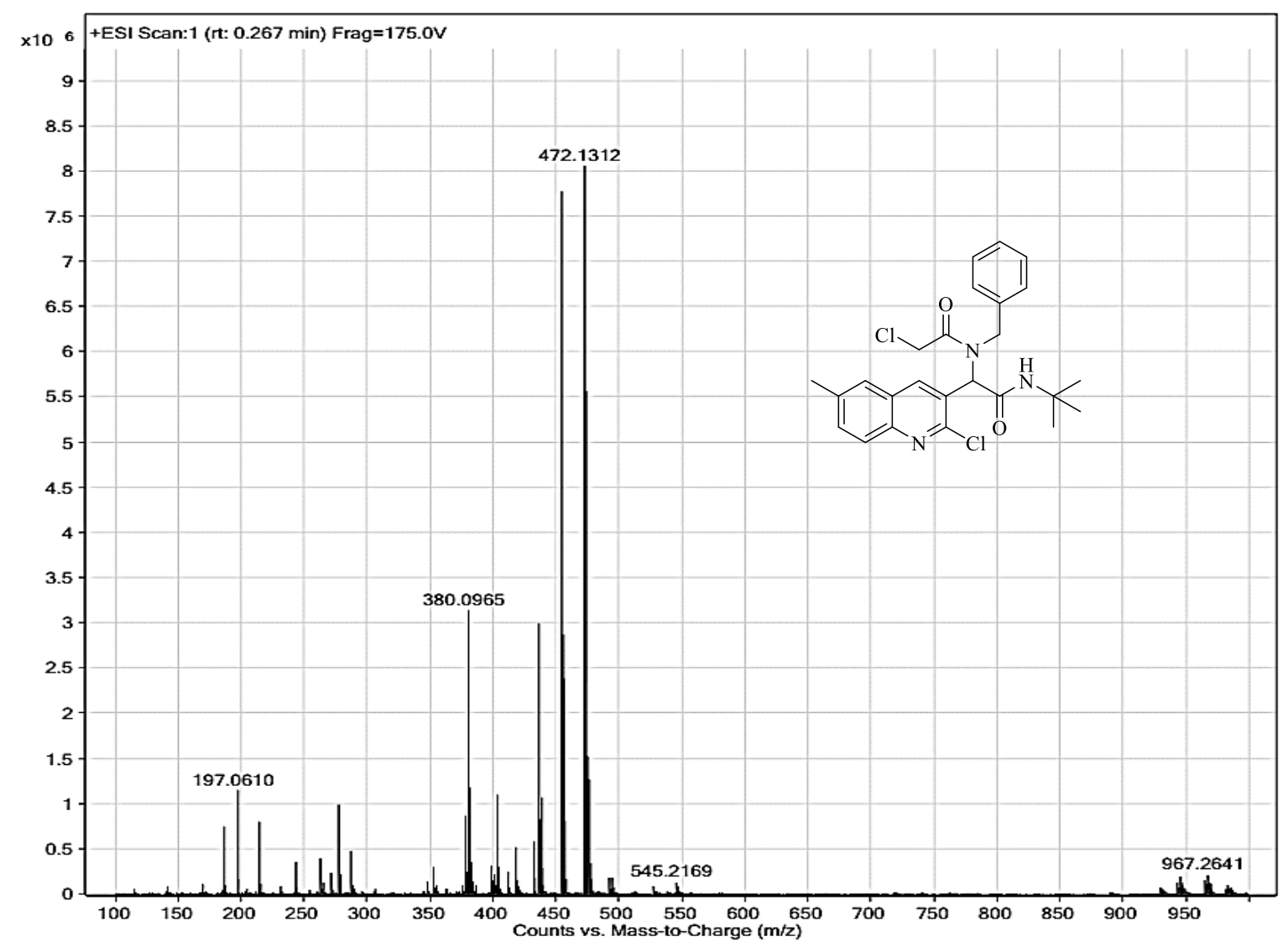

Figure S30: HRMS-ESI of $\mathbf{5 h}$ with formula $\mathrm{C}_{25} \mathrm{H}_{27} \mathrm{Cl}_{2} \mathrm{~N}_{3} \mathrm{O}_{2}$ and $[\mathrm{M}+\mathrm{H}]^{+} 472.1303$ 


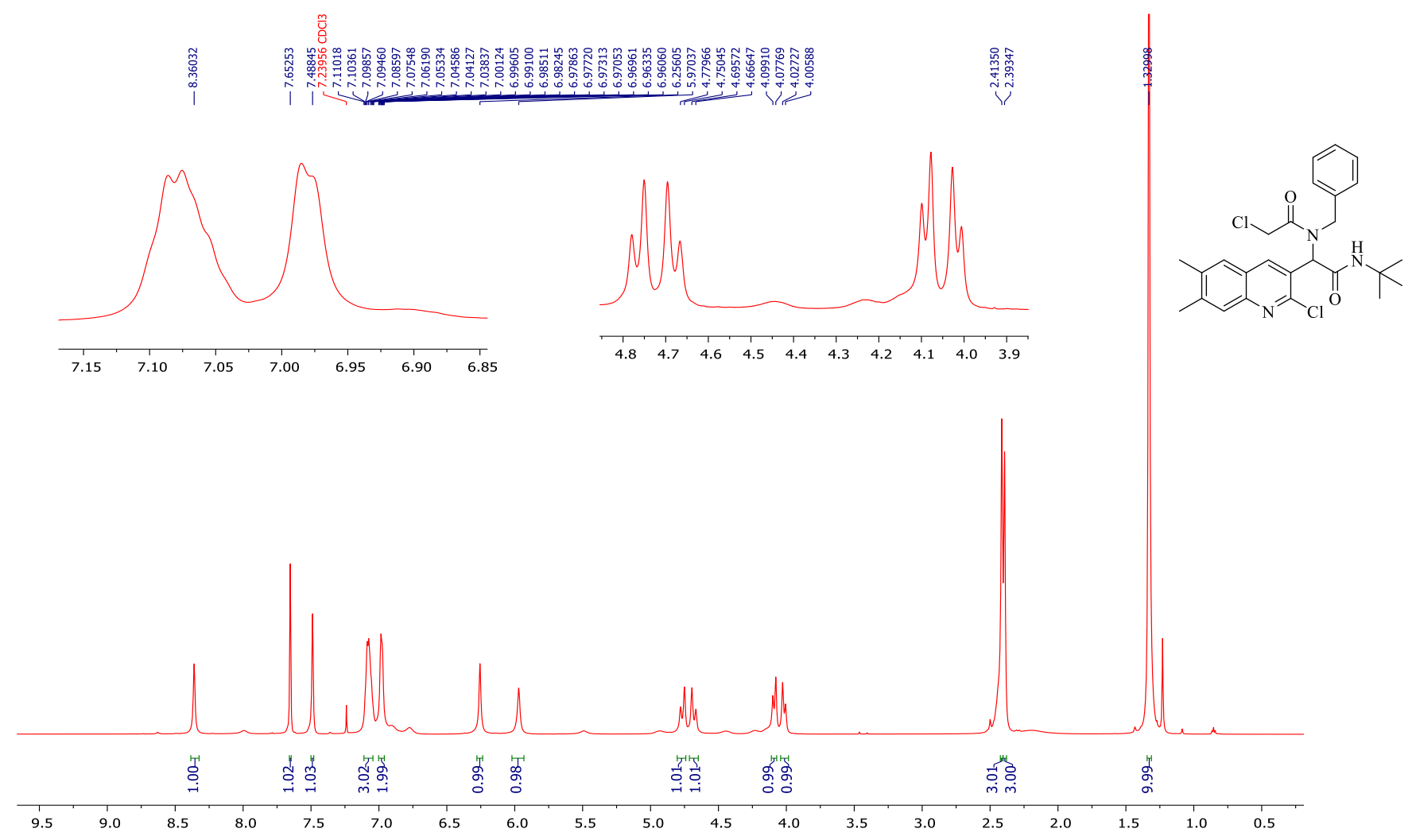

Figure S31: ${ }^{1} \mathrm{H}-\mathrm{NMR}$ of compound $\mathbf{5 i}\left(600 \mathrm{MHz}, \mathrm{CDCl}_{3}\right)$

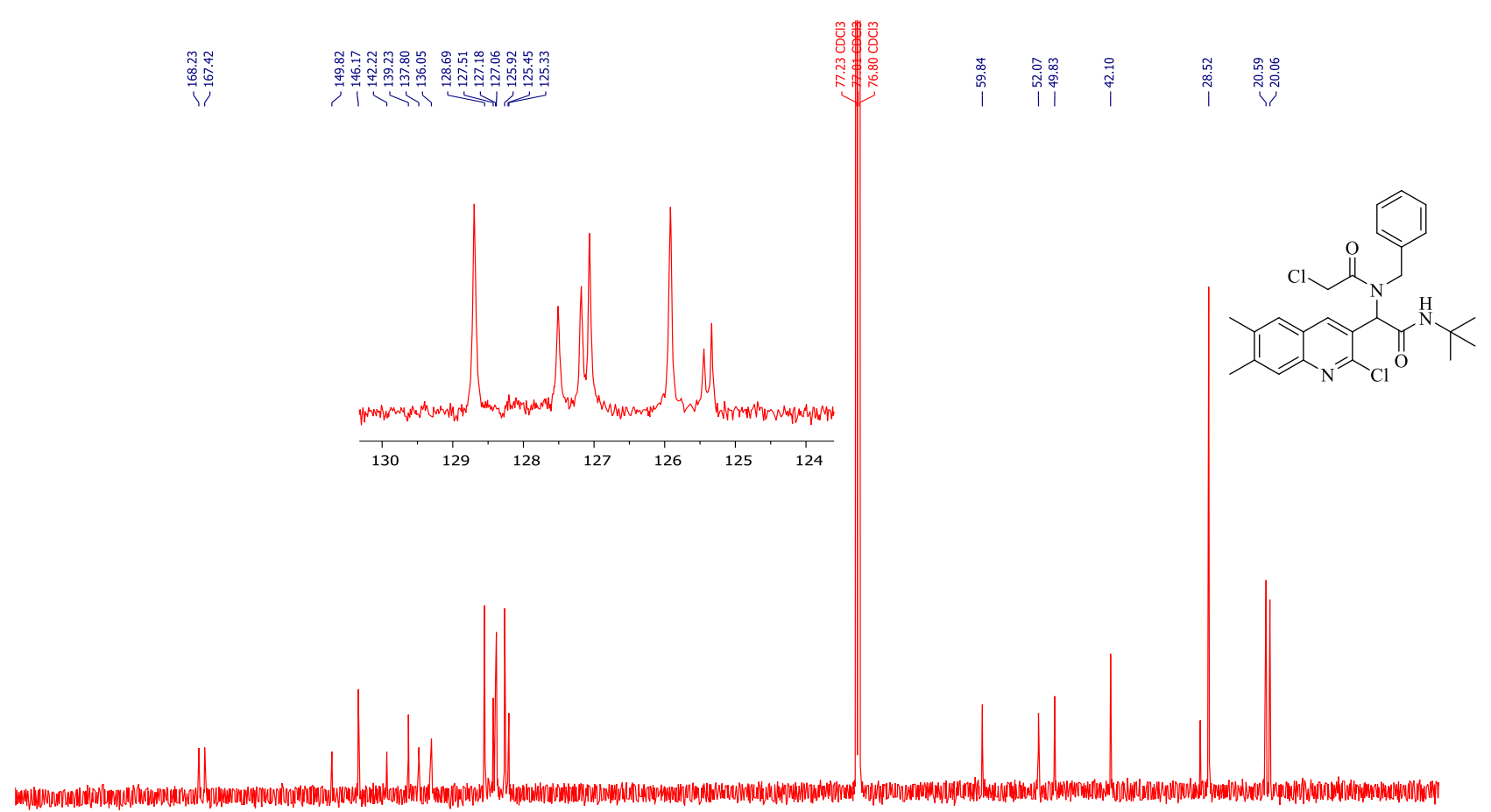

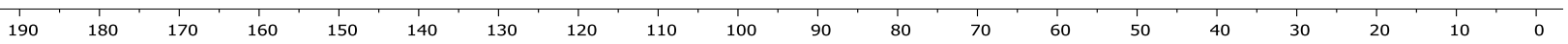

Figure S32: ${ }^{13} \mathrm{C}-\mathrm{NMR}$ of compound $5 \mathbf{i}\left(150 \mathrm{MHz}, \mathrm{CDCl}_{3}\right)$ 


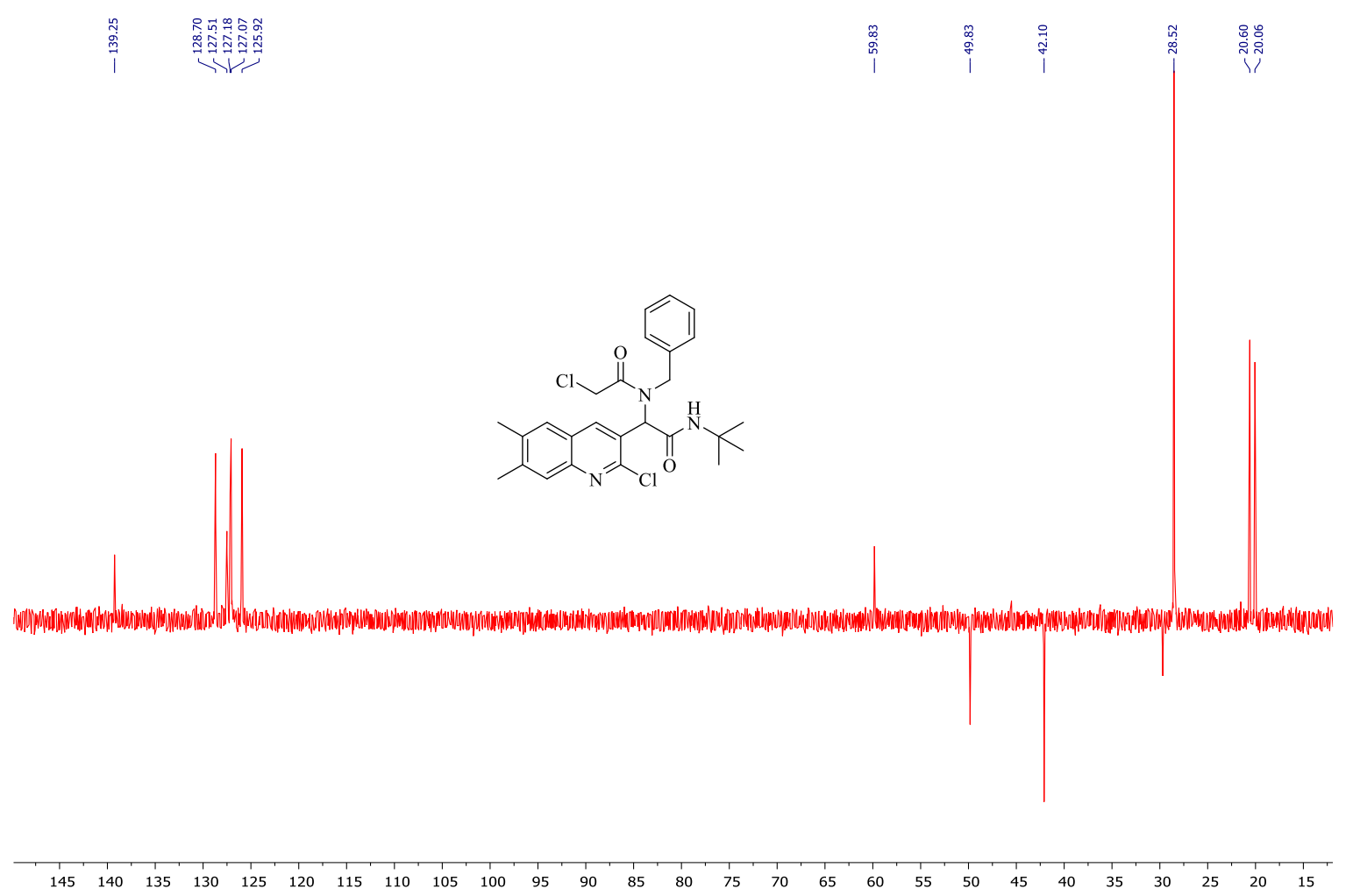

Figure S33: ${ }^{13} \mathrm{C}-\mathrm{NMR}$ (DEPT 135) of compound 5i (150 MHz, $\mathrm{CDCl}_{3}$ )

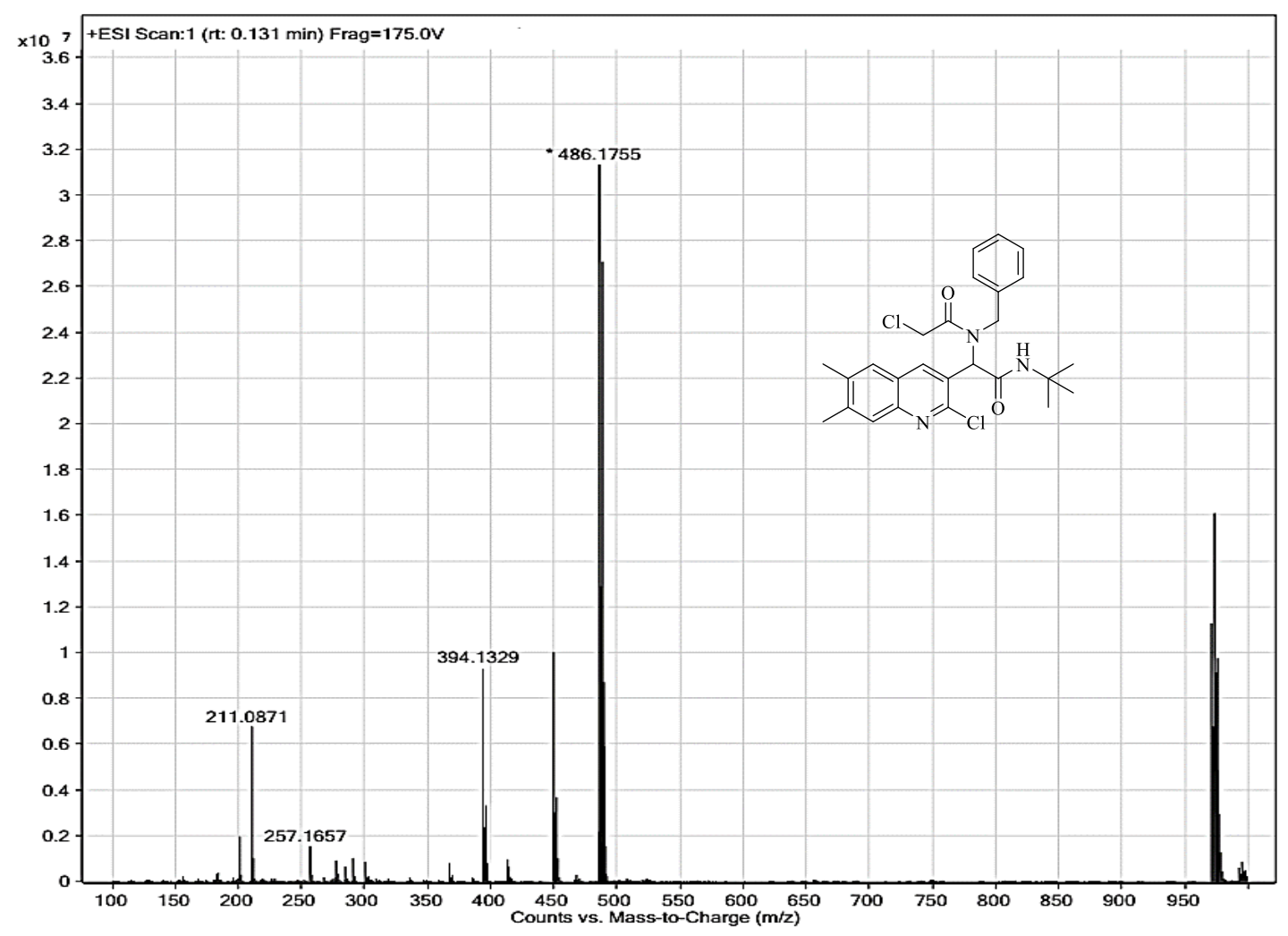

Figure S34: HRMS-ESI of 5i with formula $\mathrm{C}_{26} \mathrm{H}_{29} \mathrm{Cl}_{2} \mathrm{~N}_{3} \mathrm{O}_{2}$ and $[\mathrm{M}+\mathrm{H}]^{+} 486.1763$ 

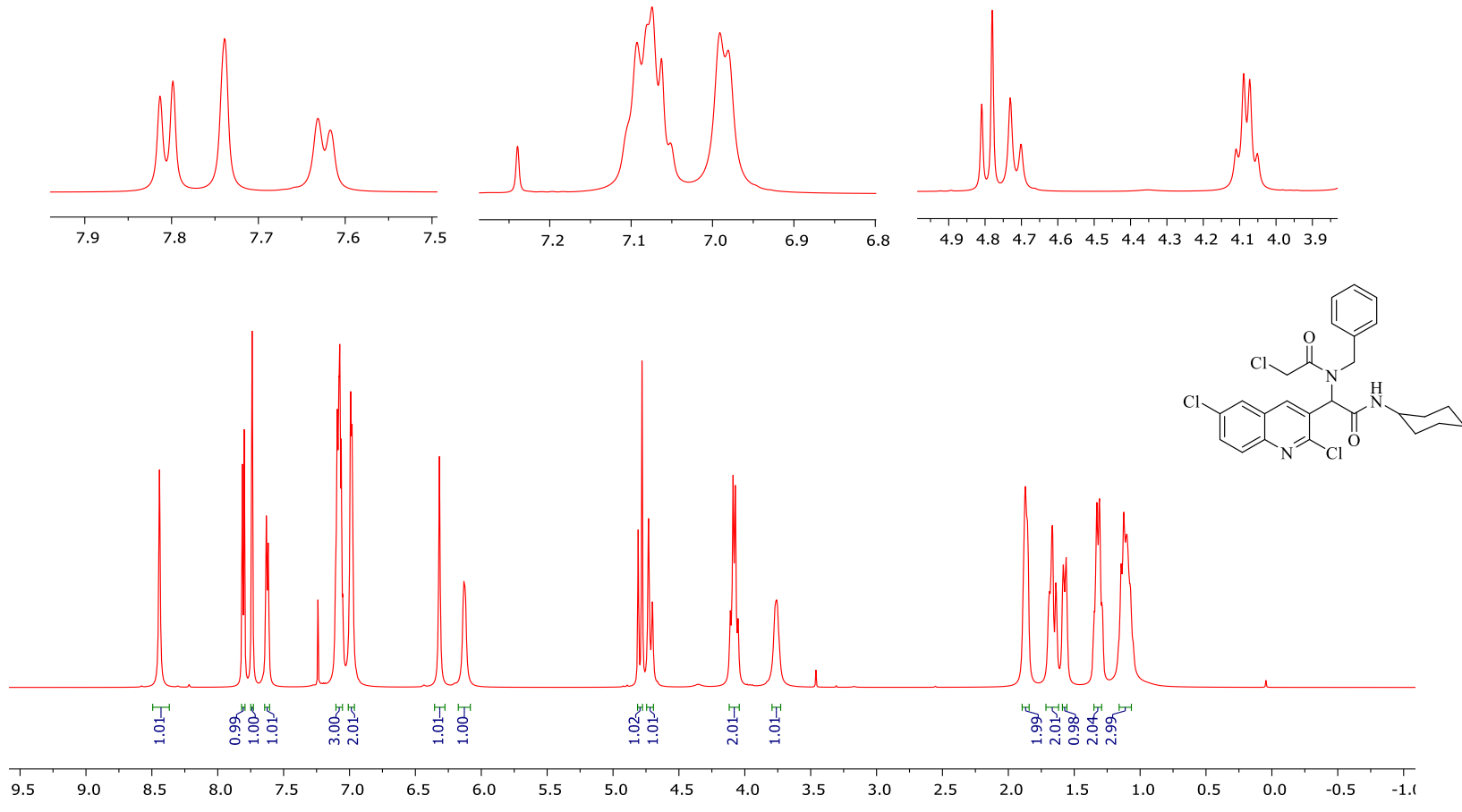

Figure S35: ${ }^{1} \mathrm{H}-\mathrm{NMR}$ of compound $\mathbf{5 j}\left(600 \mathrm{MHz}, \mathrm{CDCl}_{3}\right)$

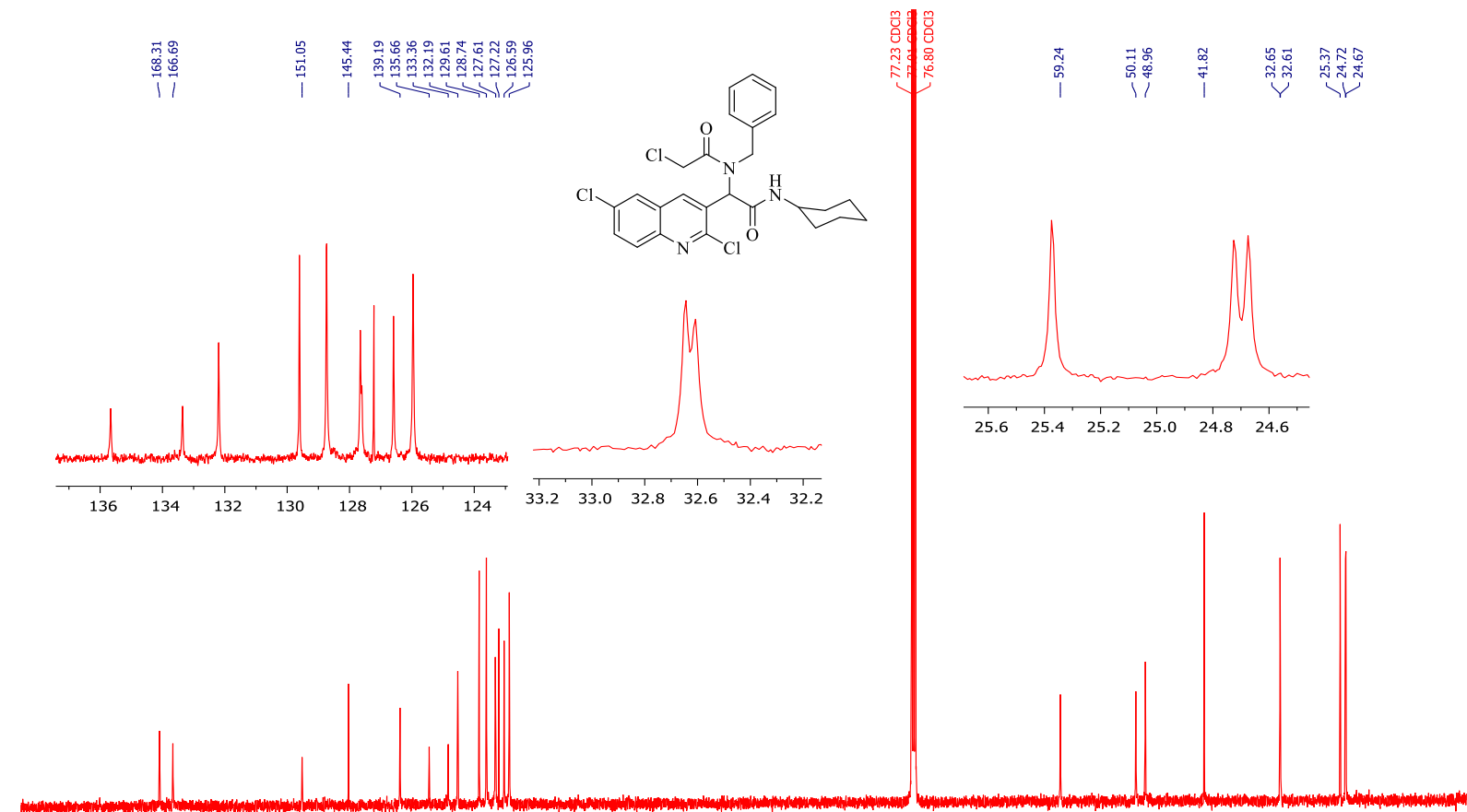

$180 \quad 170 \quad 160$

Figure S36: ${ }^{13} \mathrm{C}-\mathrm{NMR}$ of compound $\mathbf{5 j}\left(150 \mathrm{MHz}, \mathrm{CDCl}_{3}\right)$ 


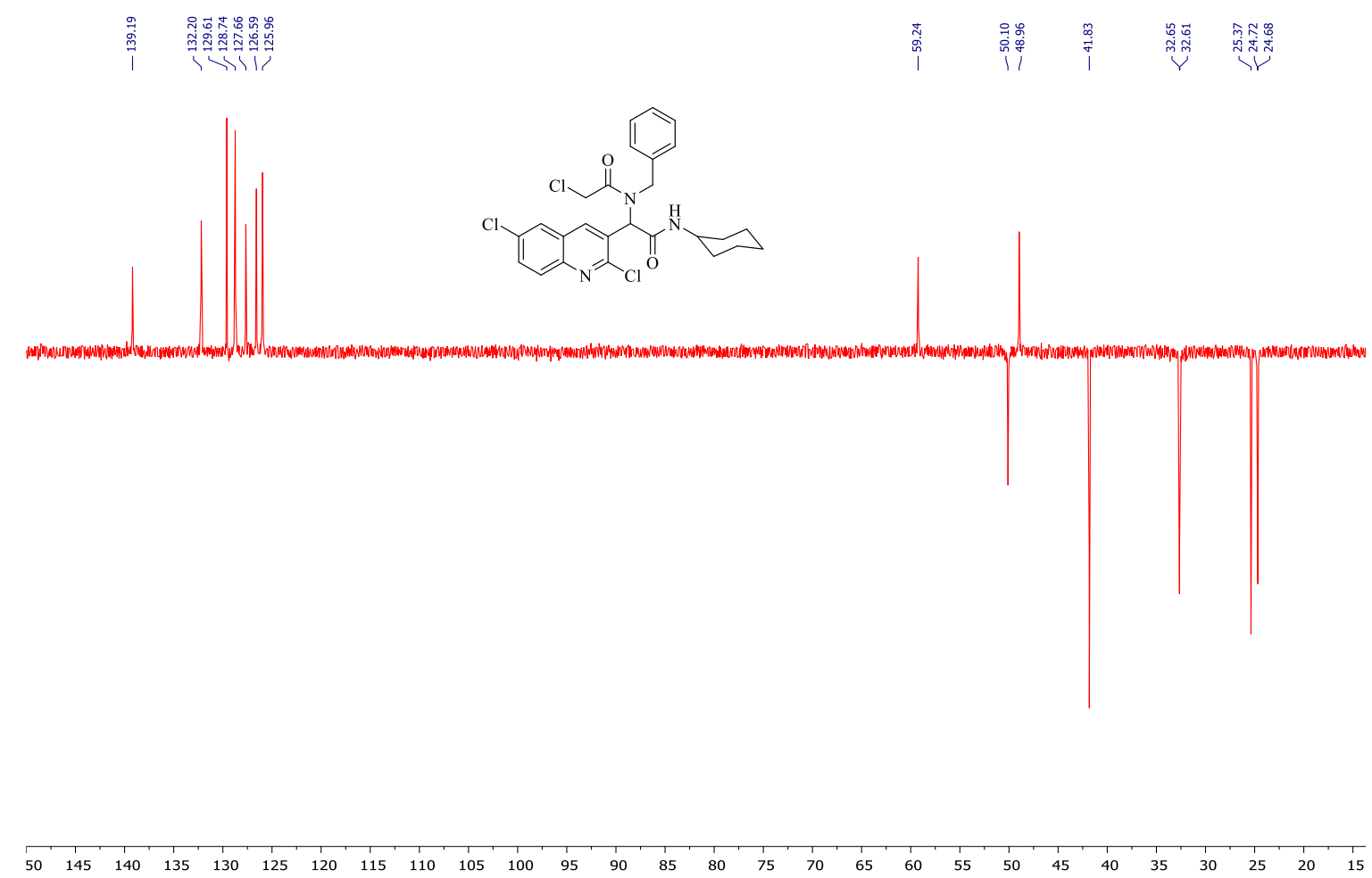

Figure S37: ${ }^{13} \mathrm{C}-\mathrm{NMR}$ (DEPT 135) of compound $\mathbf{5 j}\left(150 \mathrm{MHz}, \mathrm{CDCl}_{3}\right.$ )

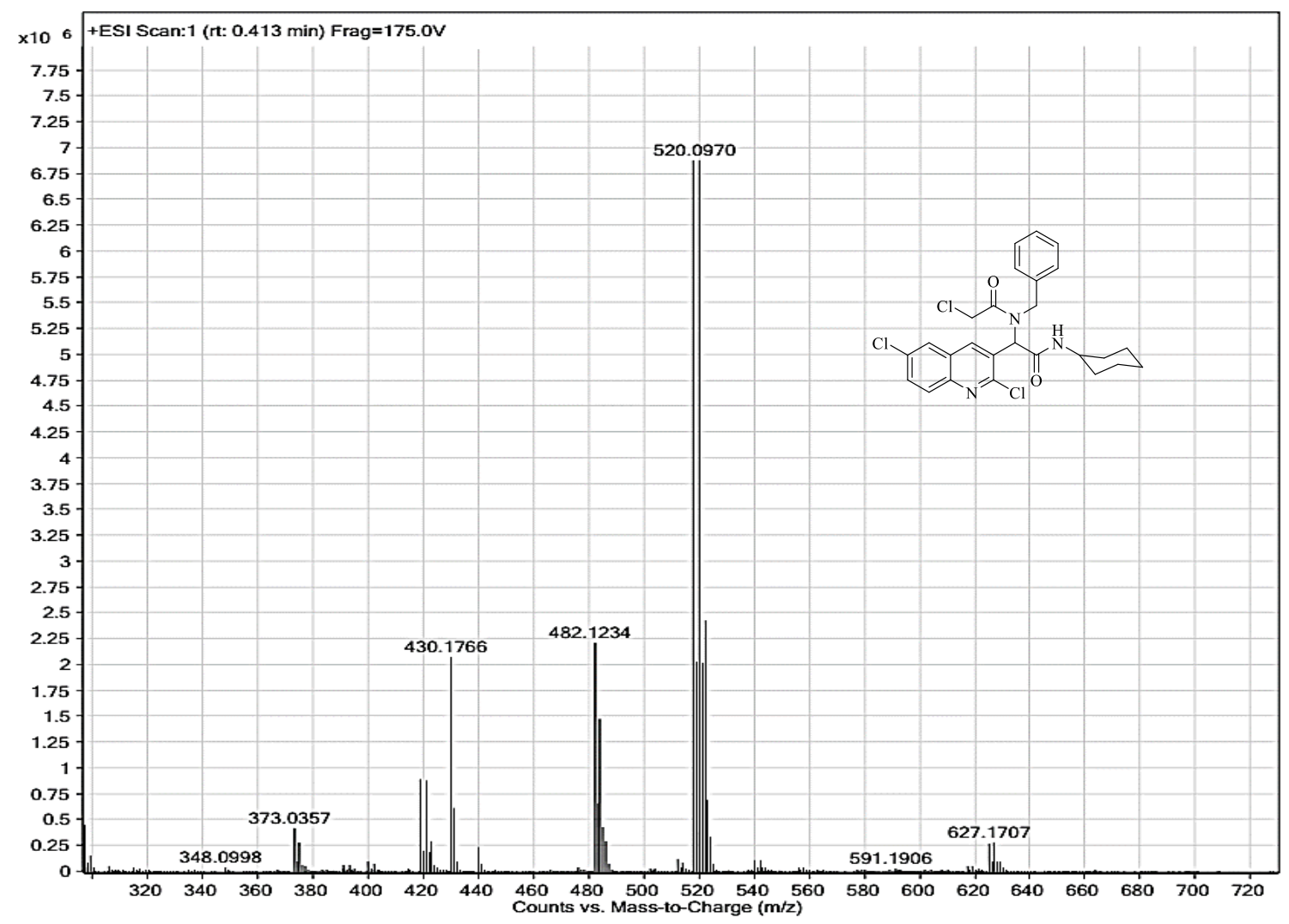

Figure S38: HRMS-ESI of $\mathbf{5 j}$ with formula $\mathrm{C}_{26} \mathrm{H}_{26} \mathrm{Cl}_{3} \mathrm{~N}_{3} \mathrm{O}_{2}$ and $[\mathrm{M}+\mathrm{H}]^{+}$518.1169. 

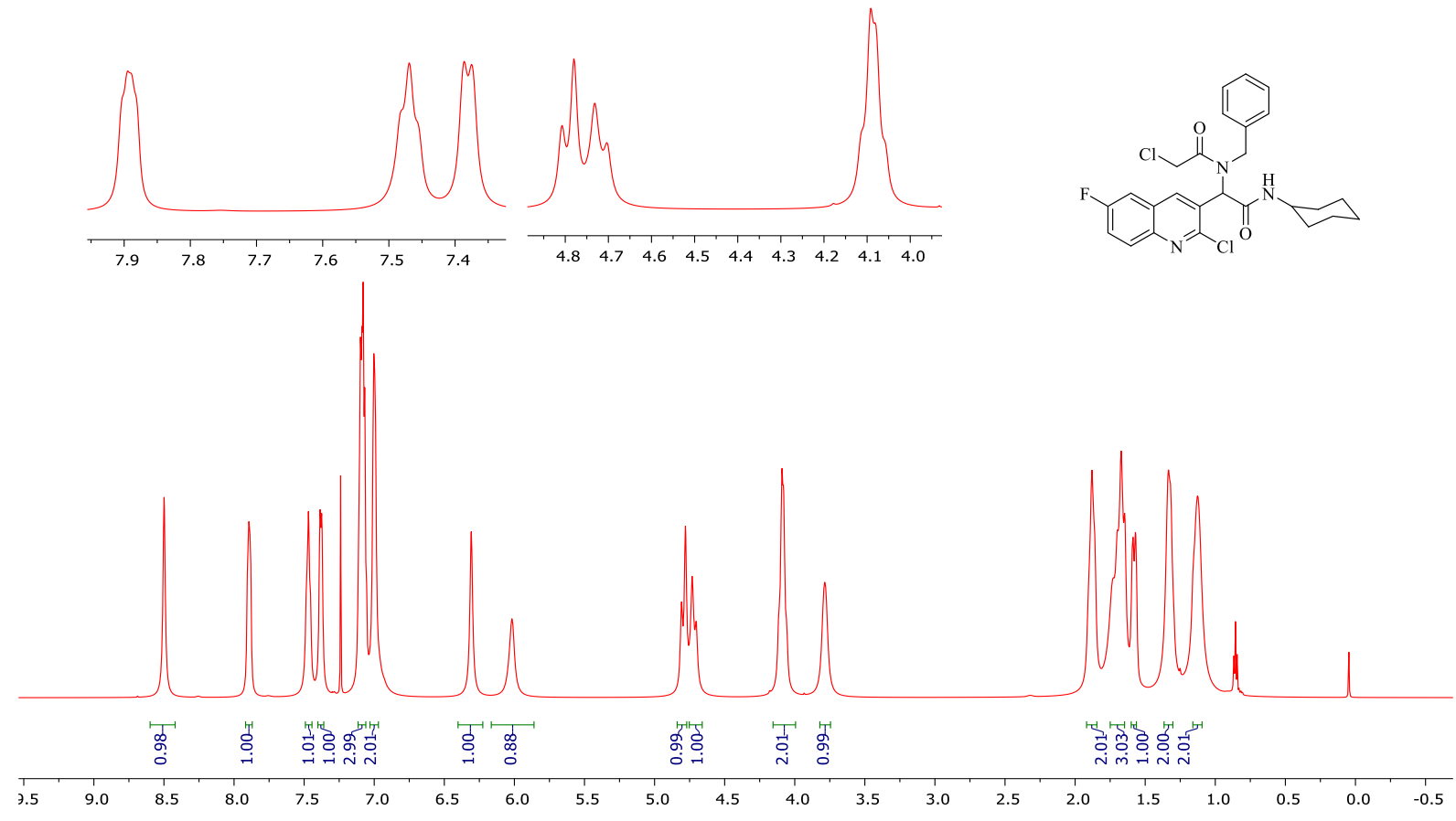

Figure S39: ${ }^{1} \mathrm{H}-\mathrm{NMR}$ of compound $\mathbf{5 k}\left(600 \mathrm{MHz}, \mathrm{CDCl}_{3}\right)$

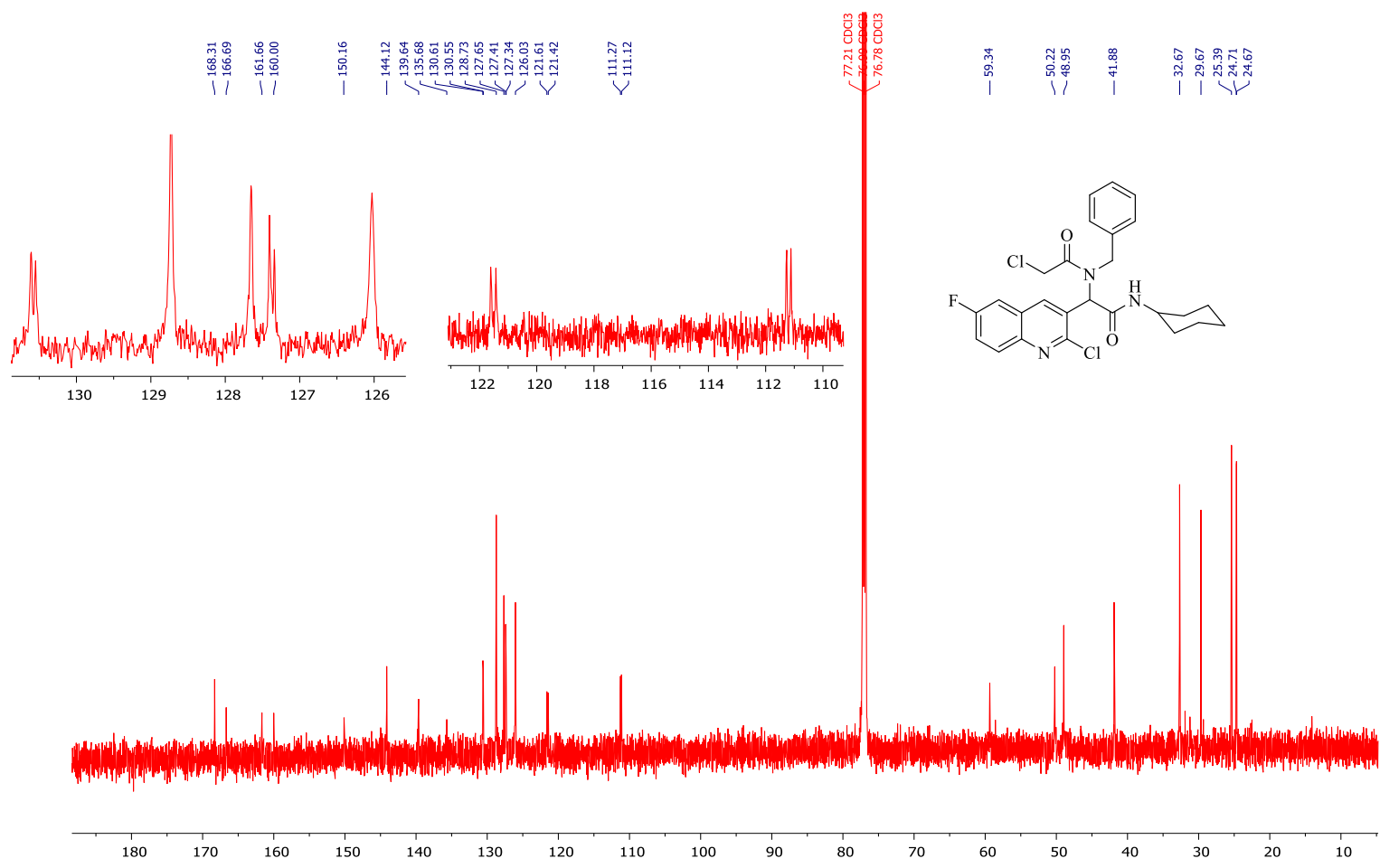

Figure S40: ${ }^{13} \mathrm{C}-\mathrm{NMR}$ of compound $\mathbf{5 k}\left(150 \mathrm{MHz}, \mathrm{CDCl}_{3}\right)$ 

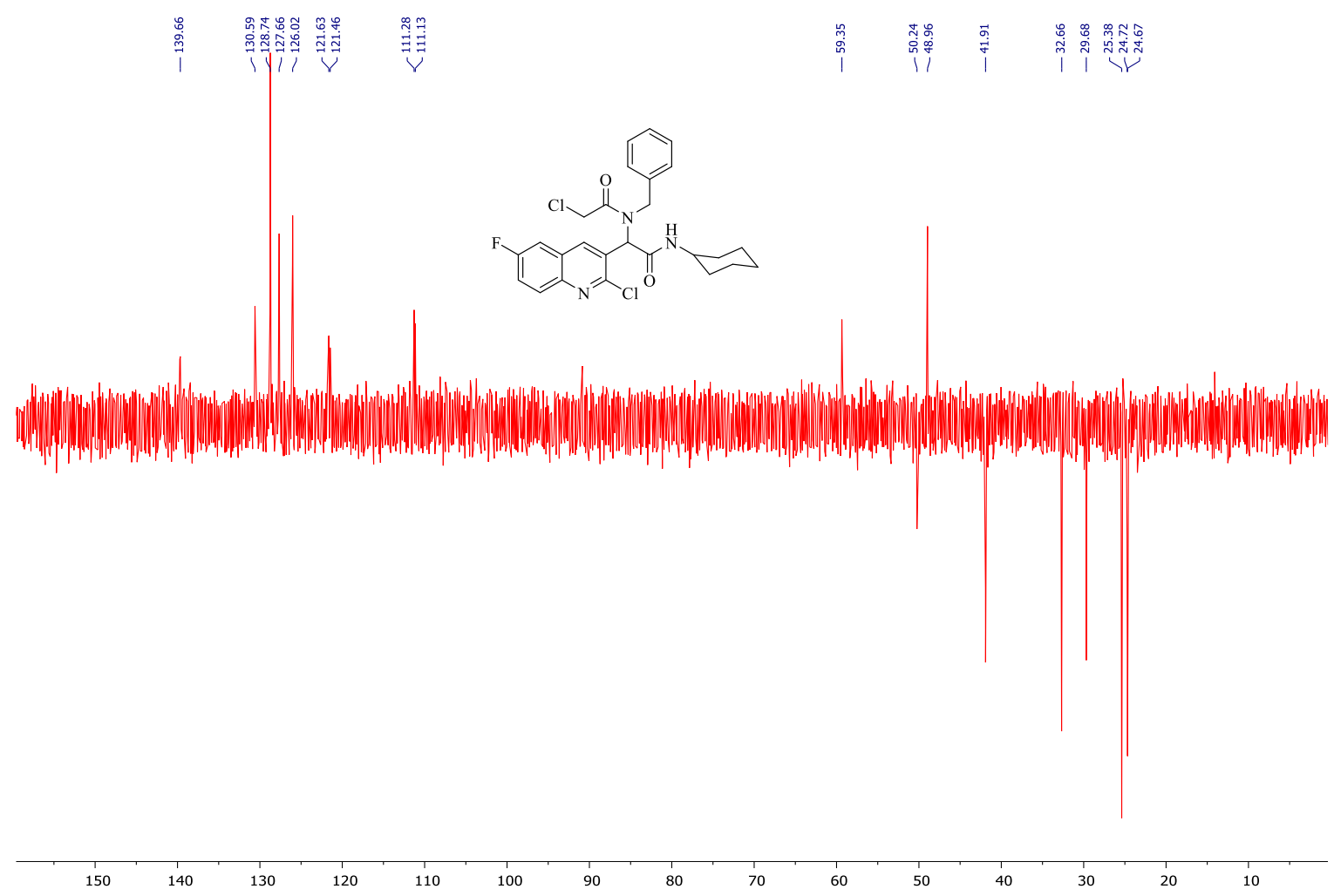

Figure S41: ${ }^{13} \mathrm{C}-\mathrm{NMR}$ (DEPT 135) of compound sk (150 MHz, $\mathrm{CDCl}_{3}$ )

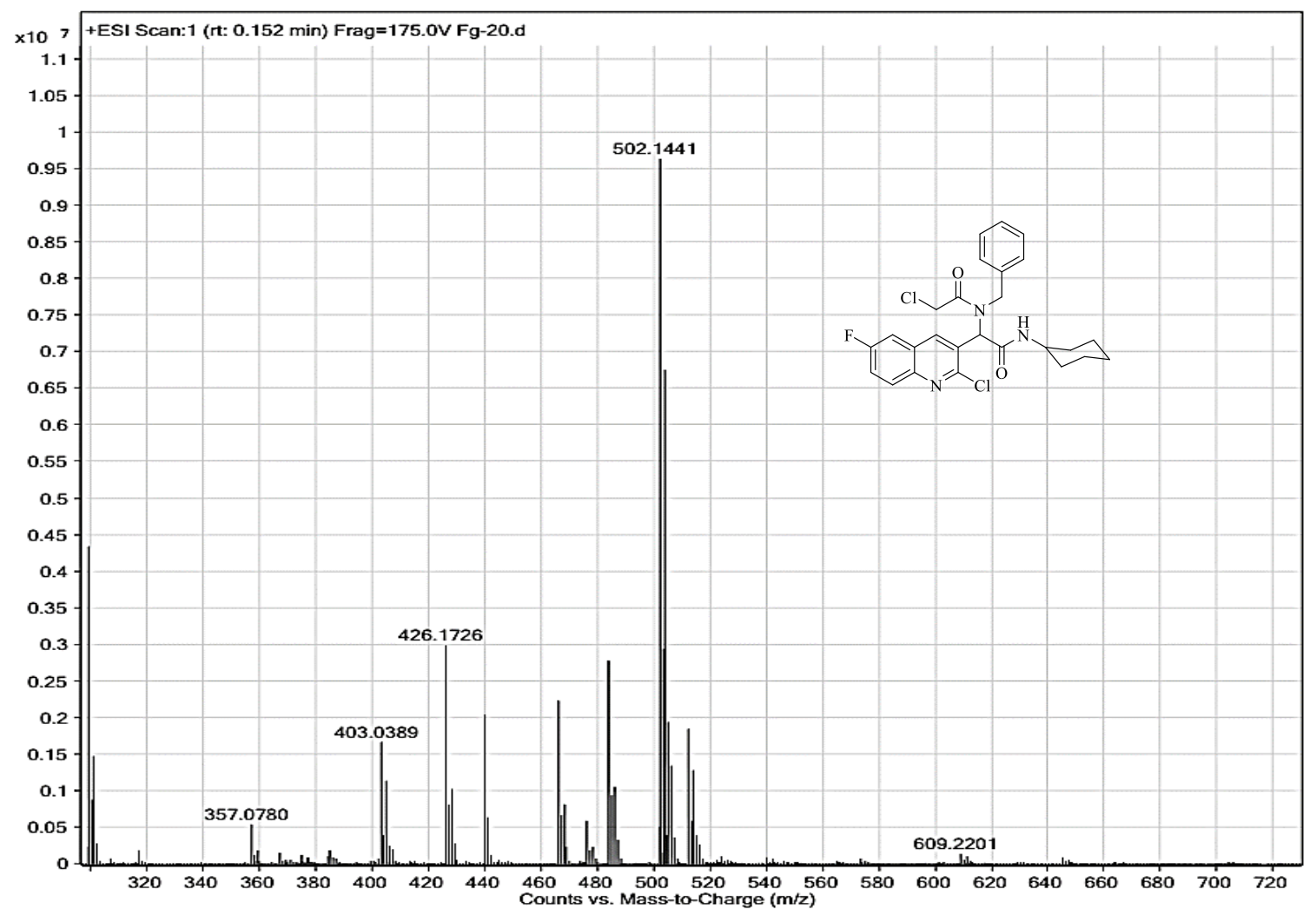

Figure S42: HRMS-ESI of sk with formula $\mathrm{C}_{26} \mathrm{H}_{26} \mathrm{Cl}_{2} \mathrm{FN}_{3} \mathrm{O}_{2}$ and $[\mathrm{M}+\mathrm{H}]^{+} 502.1448$

S23 


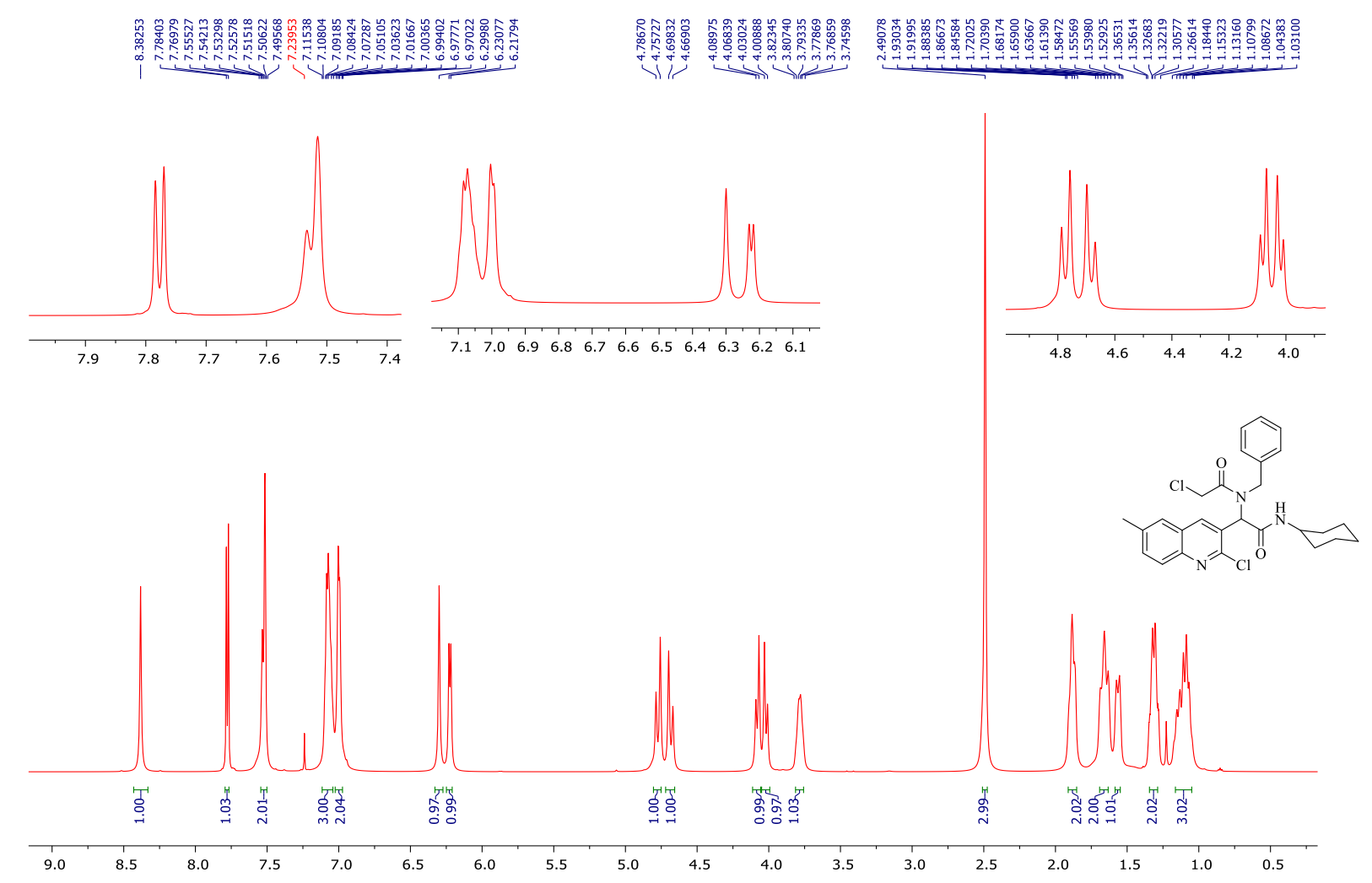

Figure S43: ${ }^{1} \mathrm{H}-\mathrm{NMR}$ of compound $\mathbf{5 l}\left(600 \mathrm{MHz}, \mathrm{CDCl}_{3}\right)$

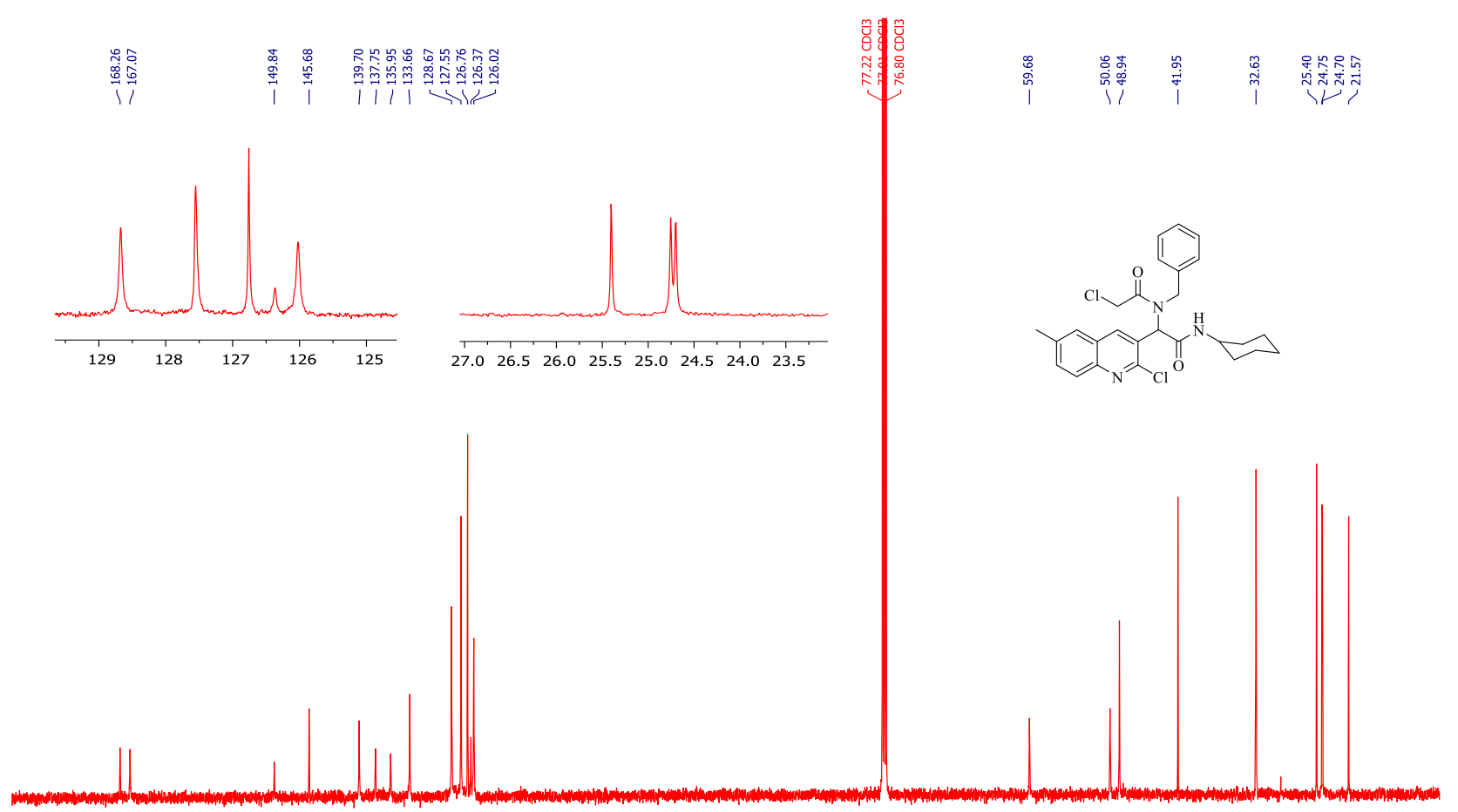

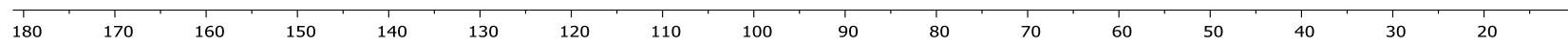

Figure S44: ${ }^{13} \mathrm{C}-\mathrm{NMR}$ of compound $\mathbf{5 l}\left(150 \mathrm{MHz}, \mathrm{CDCl}_{3}\right)$ 


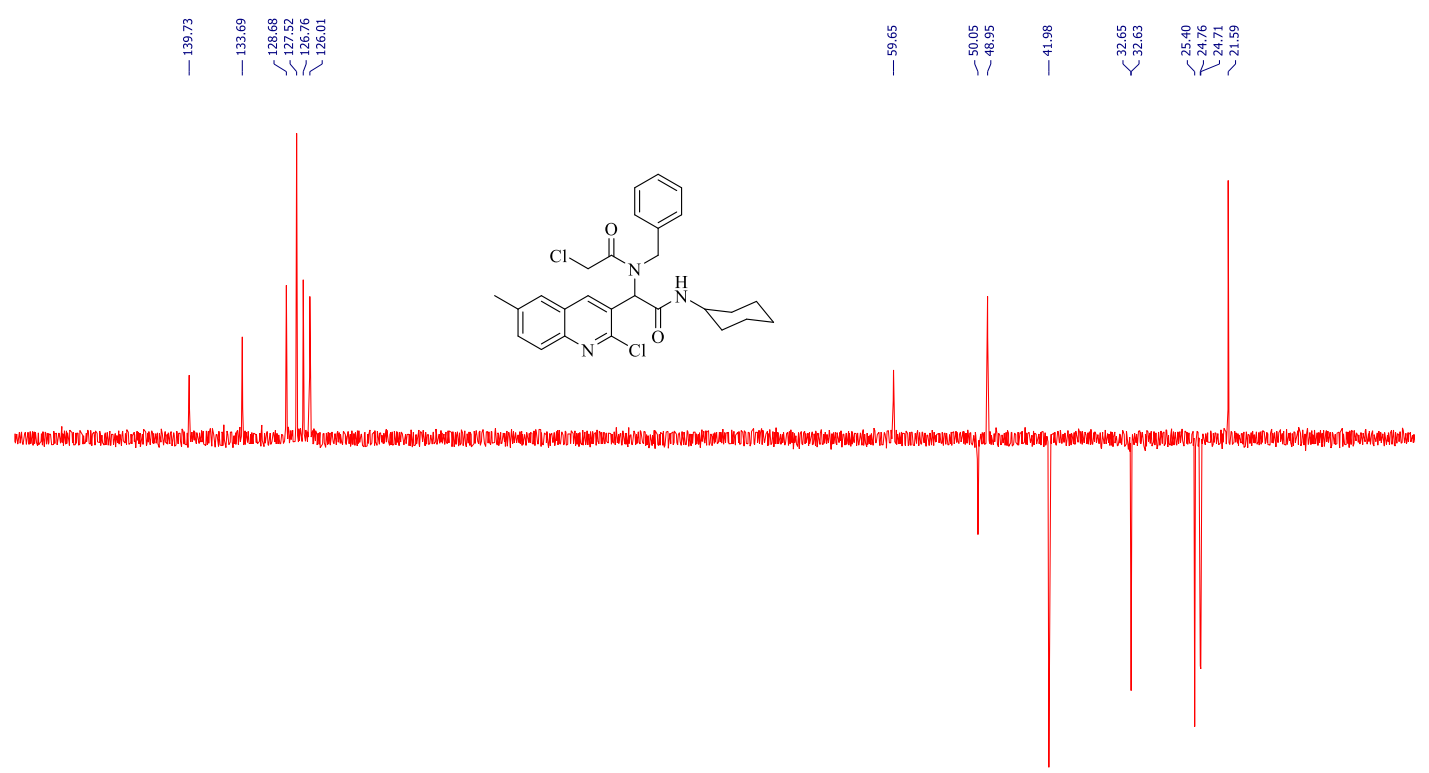

\begin{tabular}{lllllllllllllll}
\hline 1 & 1 \\
\hline 150 & 140 & 130 & 120 & 110 & 100 & 90 & 80 & 70 & 60 & 50 & 40 & 30 & 20 & 10
\end{tabular}

Figure S45: ${ }^{13} \mathrm{C}-\mathrm{NMR}$ (DEPT 135) of compound $\mathbf{5 l}\left(150 \mathrm{MHz}, \mathrm{CDCl}_{3}\right)$

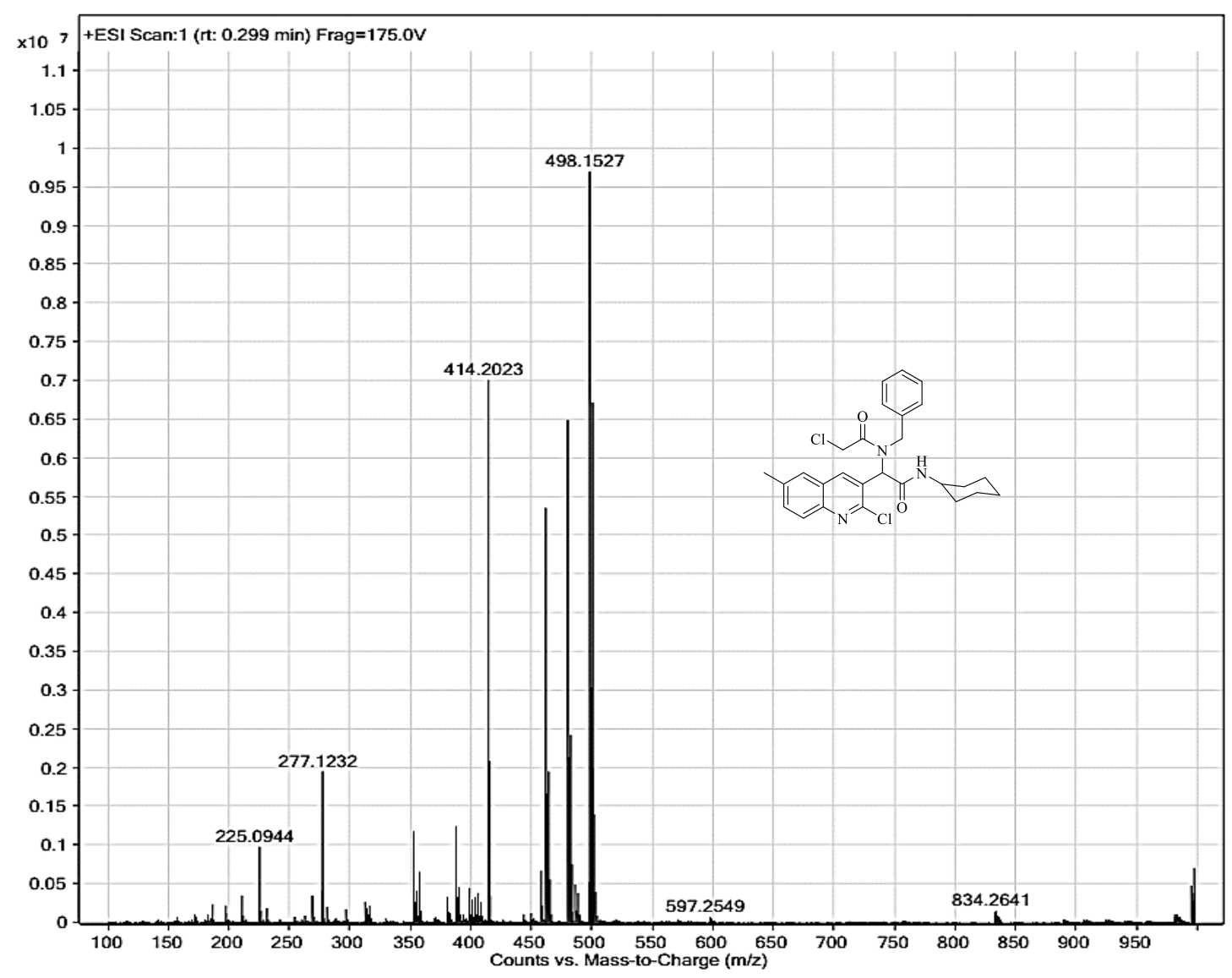

Figure S46: HRMS-ESI of $\mathbf{5 l}$ with formula $\mathrm{C}_{27} \mathrm{H}_{29} \mathrm{Cl}_{2} \mathrm{~N}_{3} \mathrm{O}_{2}$ and $[\mathrm{M}+\mathrm{H}]^{+} 498.1535$ 


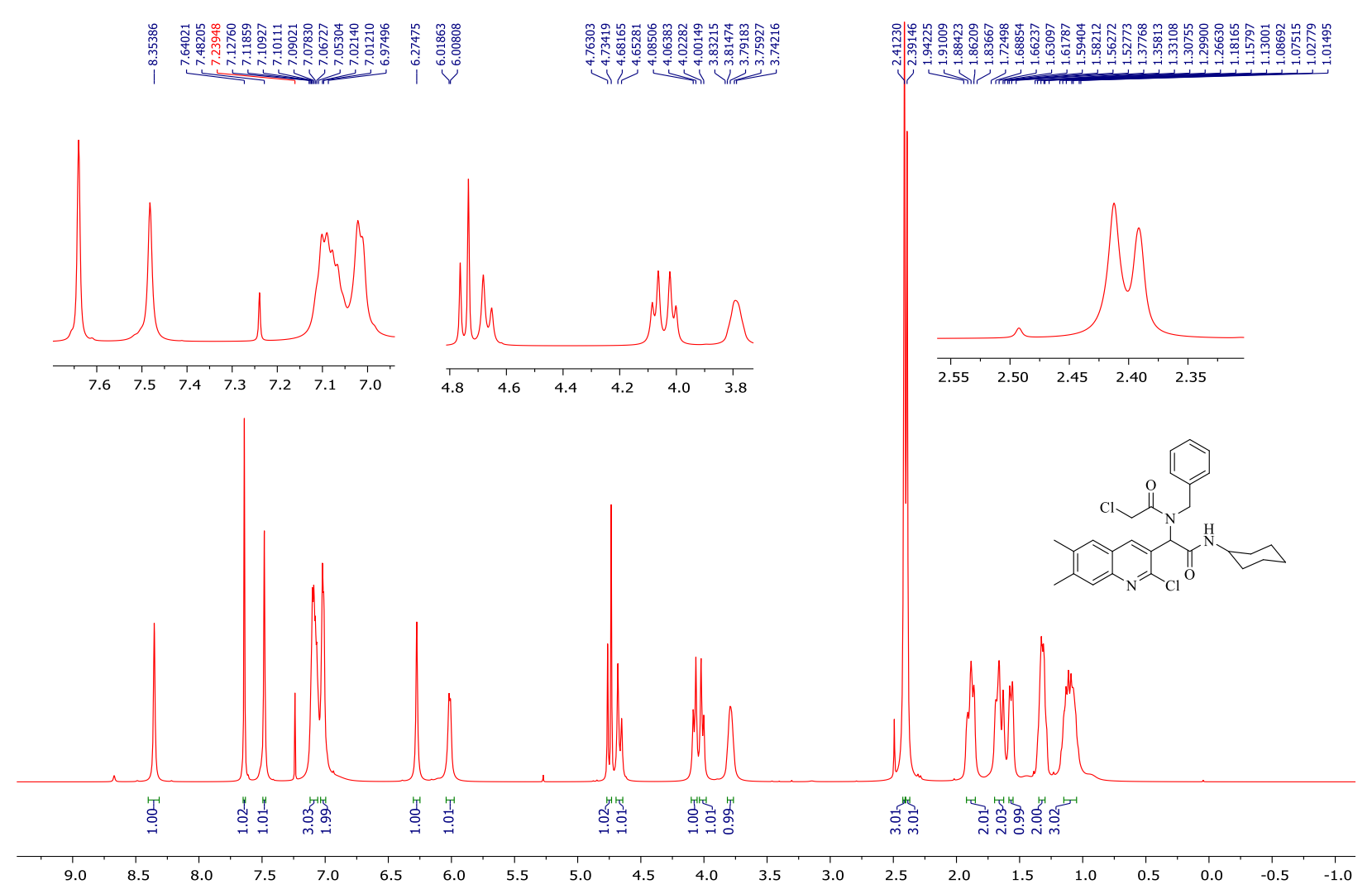

Figure S47: ${ }^{1} \mathrm{H}-\mathrm{NMR}$ of compound $\mathbf{5 m}\left(600 \mathrm{MHz}, \mathrm{CDCl}_{3}\right)$

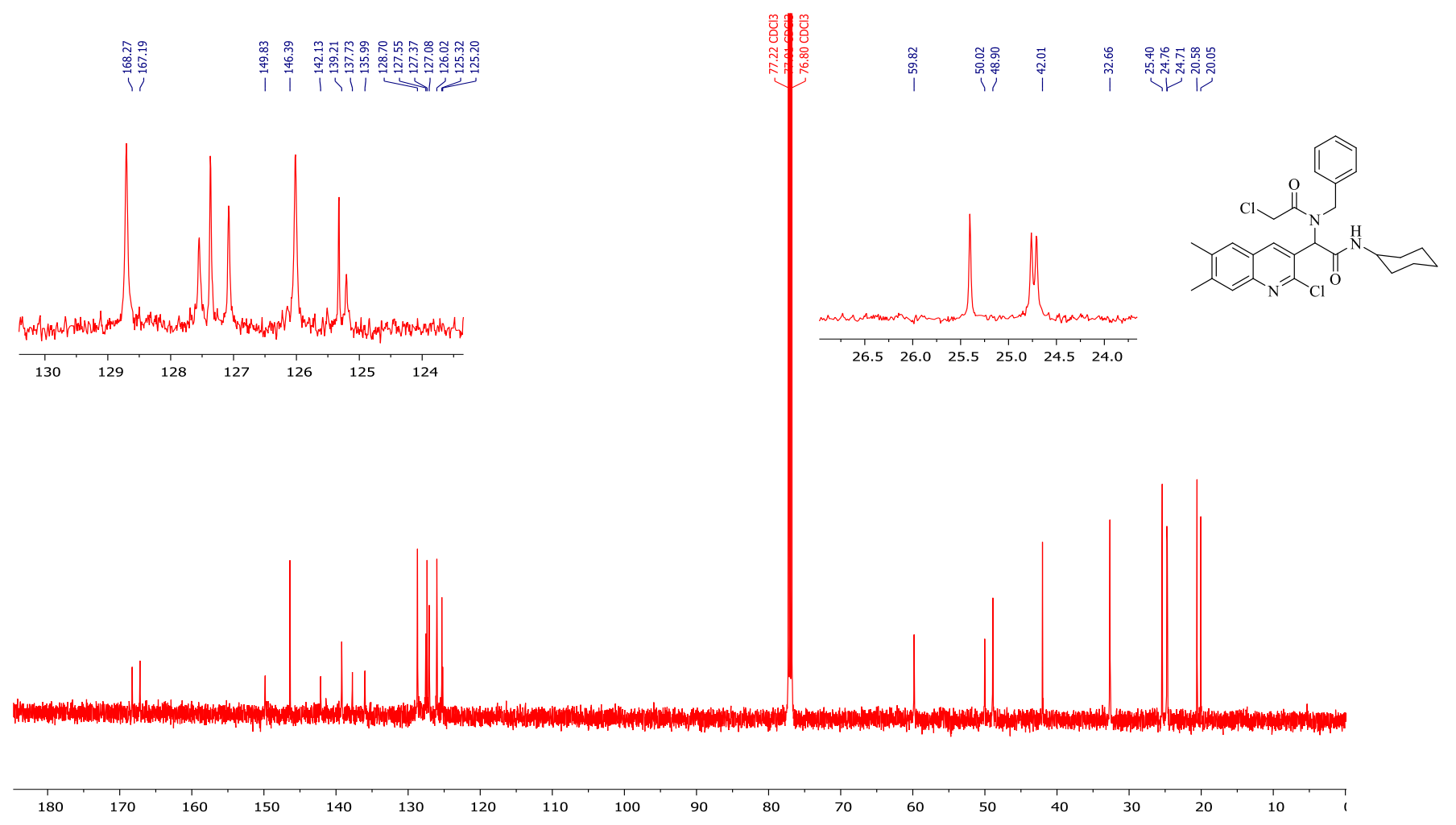

Figure S48: ${ }^{13} \mathrm{C}-\mathrm{NMR}$ of compound $\mathbf{5 m}\left(150 \mathrm{MHz}, \mathrm{CDCl}_{3}\right)$ 

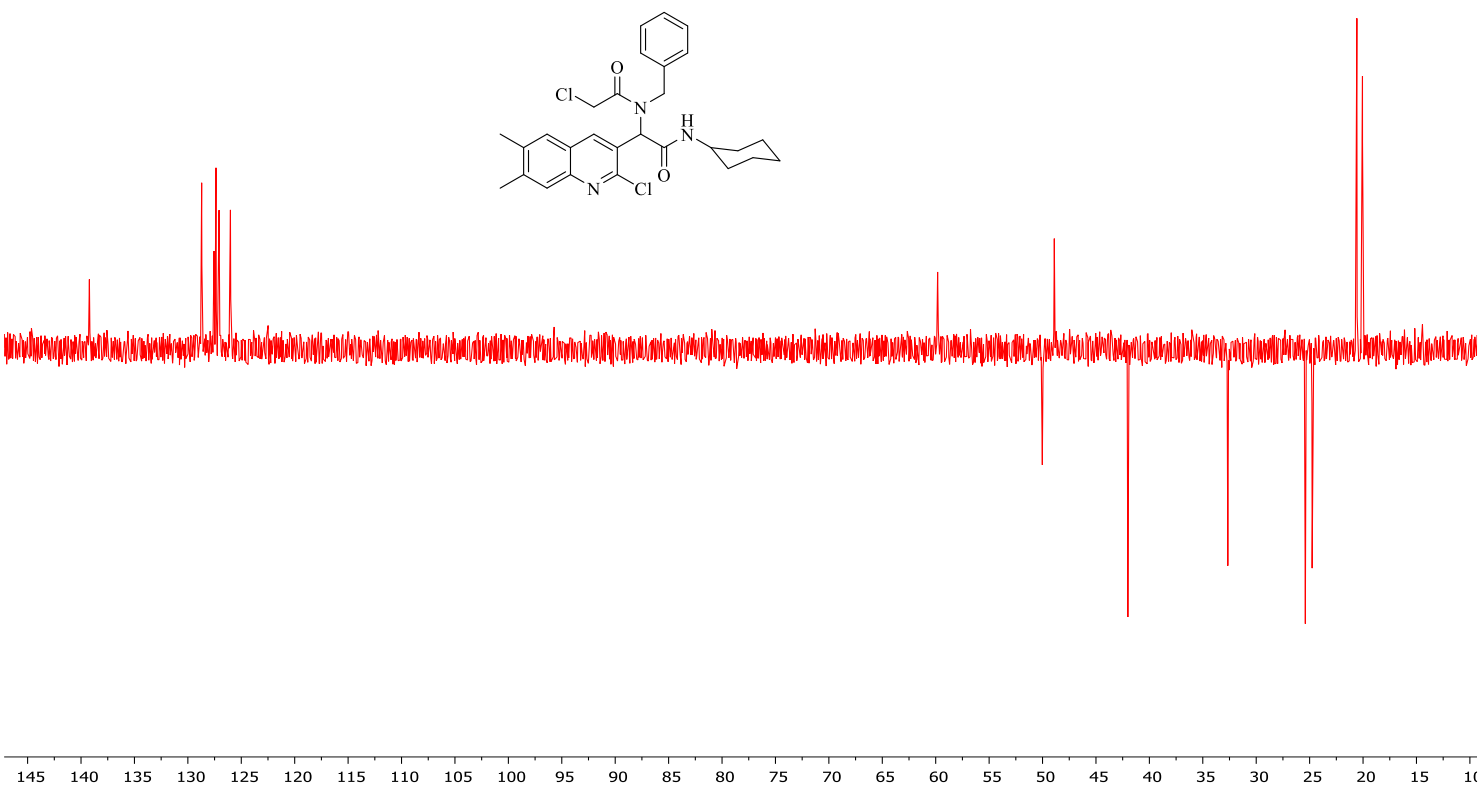

Figure S49: ${ }^{13} \mathrm{C}-\mathrm{NMR}$ (DEPT 135) of compound $5 \mathbf{m}\left(150 \mathrm{MHz}, \mathrm{CDCl}_{3}\right)$

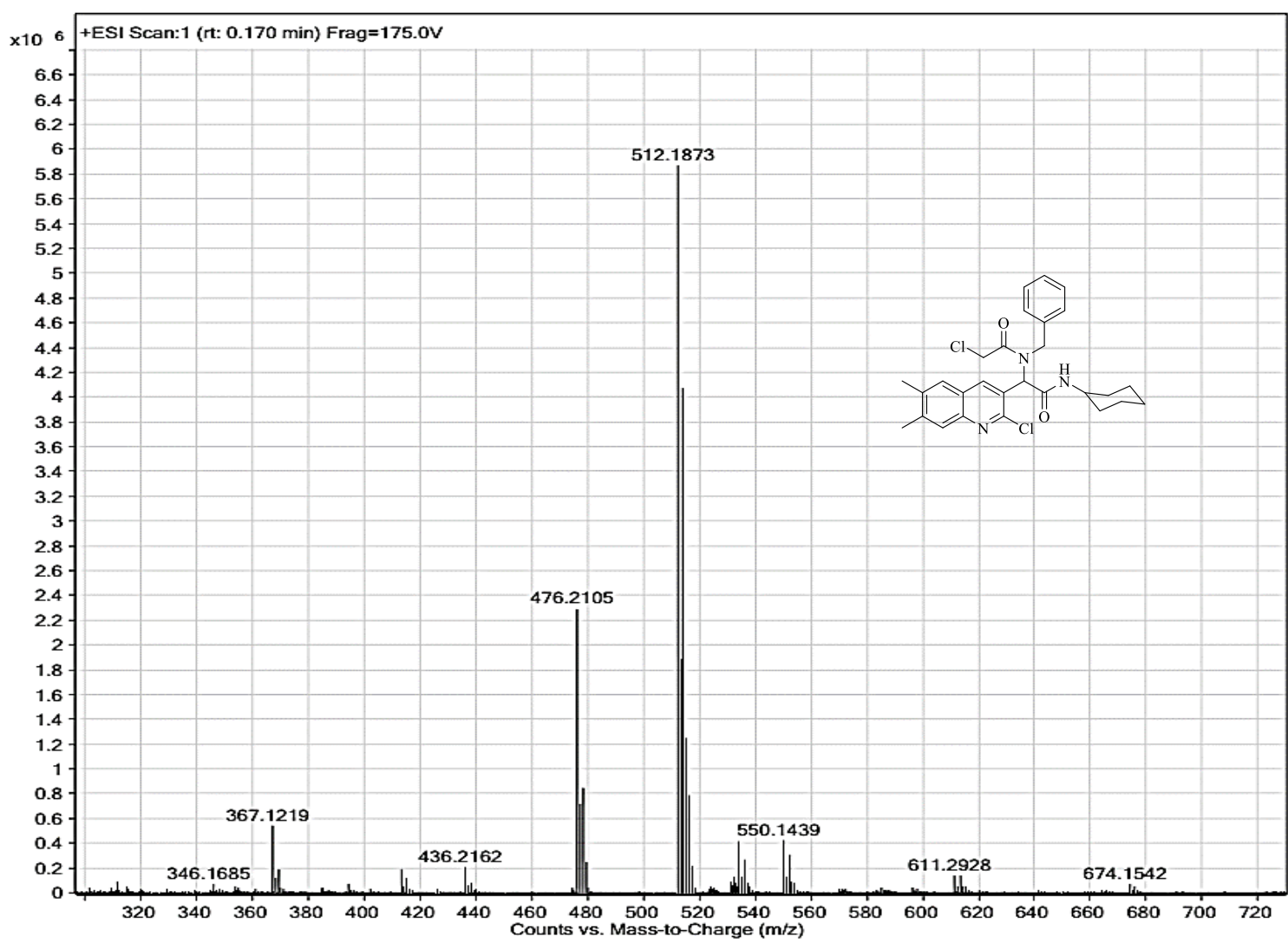

Figure S50: HRMS-ESI of $\mathbf{5 m}$ with formula $\mathrm{C}_{28} \mathrm{H}_{31} \mathrm{Cl}_{2} \mathrm{~N}_{3} \mathrm{O}_{2}$ and $[\mathrm{M}+\mathrm{H}]^{+}$512.1870; and $\mathrm{C}_{28} \mathrm{H}_{31} \mathrm{Cl}_{2} \mathrm{KN}_{3} \mathrm{O}_{2}[\mathrm{M}+\mathrm{K}]^{+} 550.1430$. 


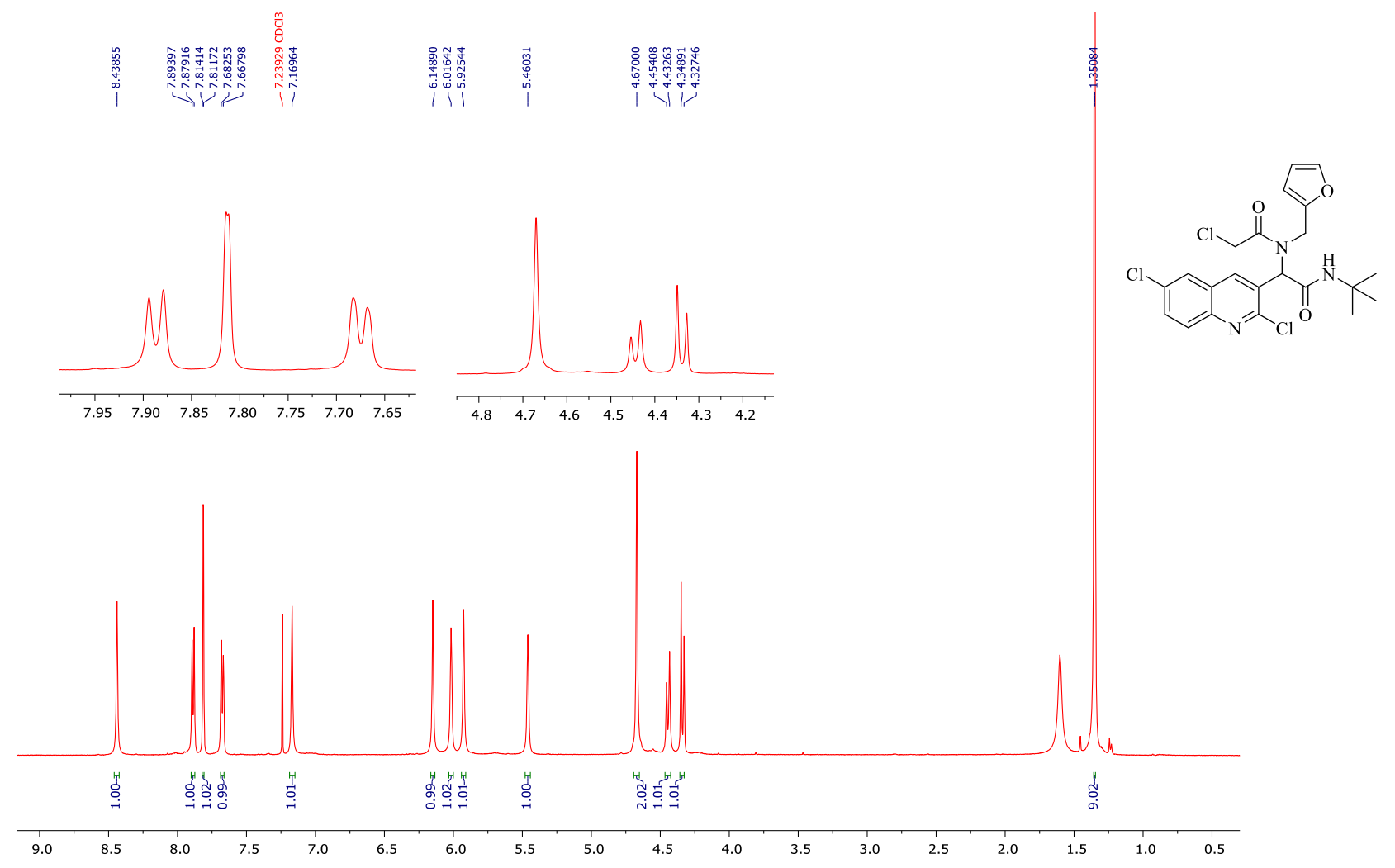

Figure S51: ${ }^{1} \mathrm{H}-\mathrm{NMR}$ of compound $\mathbf{5 n}\left(600 \mathrm{MHz}, \mathrm{CDCl}_{3}\right)$

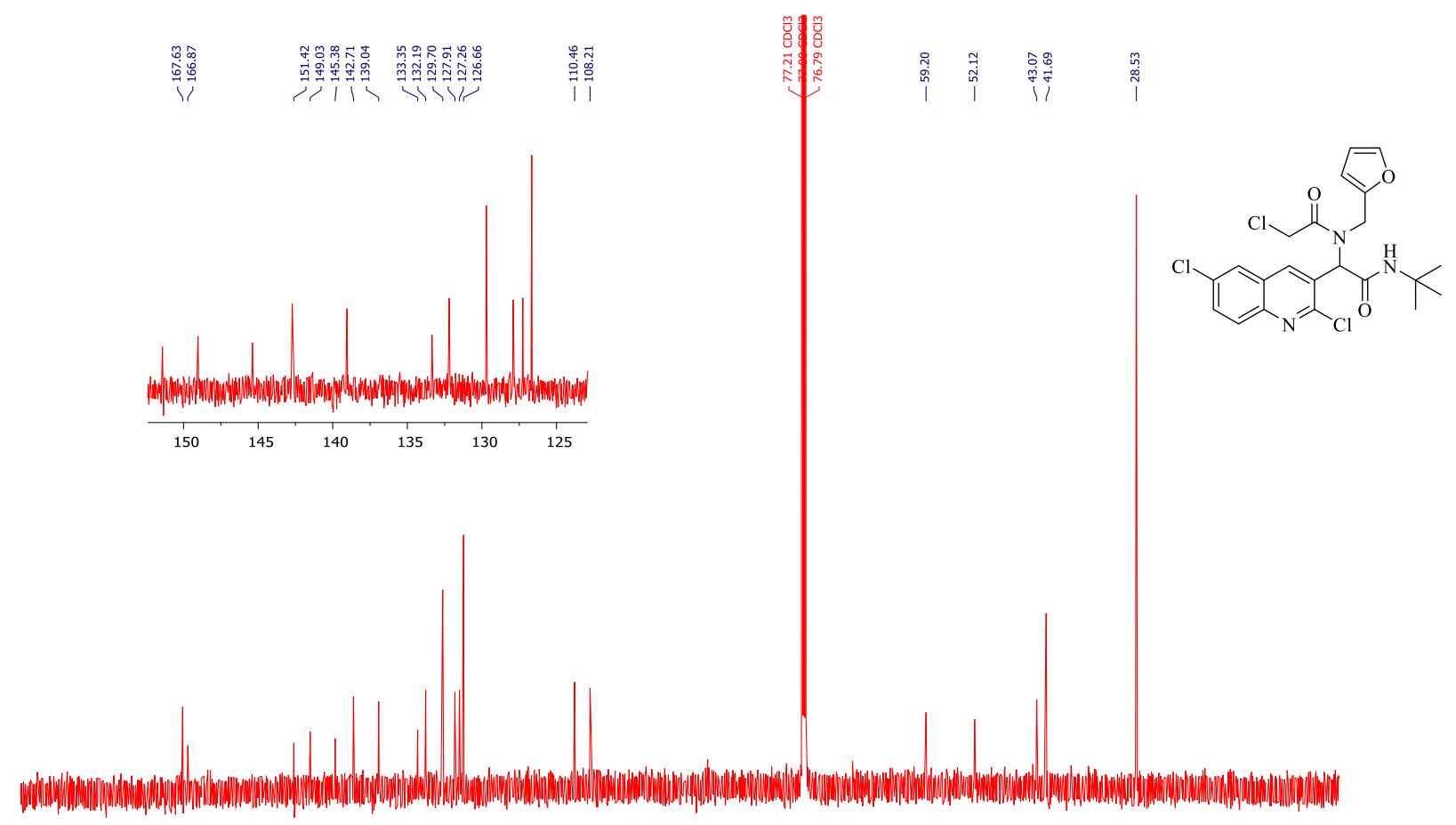

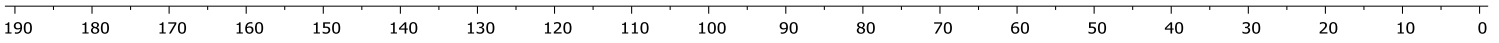

Figure S52: ${ }^{13} \mathrm{C}-\mathrm{NMR}$ of compound 5 n $\left(150 \mathrm{MHz}, \mathrm{CDCl}_{3}\right)$ 


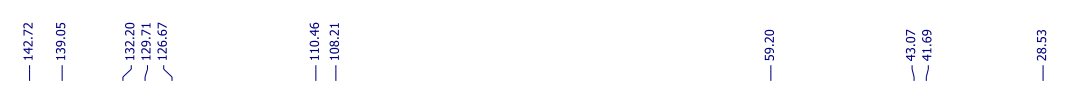<smiles>CC(C)(C)NC(=O)C(c1cc2cc(Cl)ccc2nc1Cl)C(Cc1ccco1)C(=O)CCl</smiles>

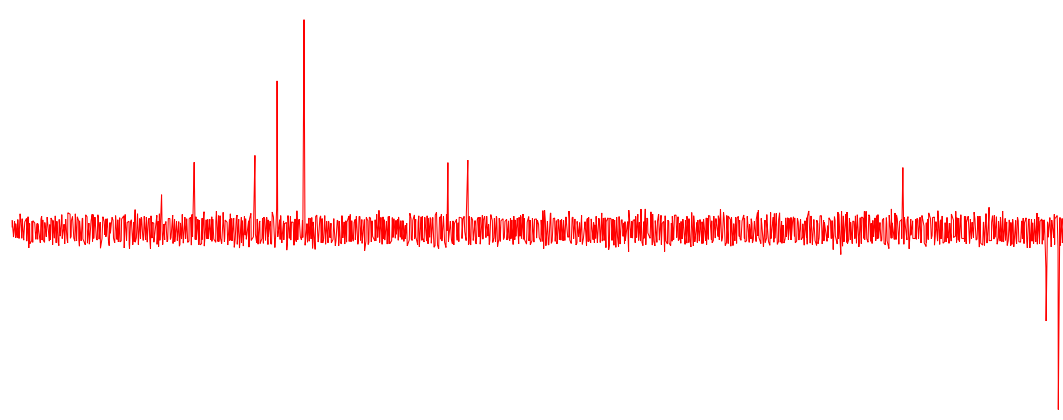

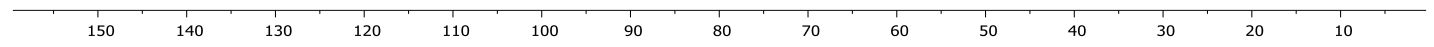

Figure S53: ${ }^{13} \mathrm{C}-\mathrm{NMR}$ (DEPT 135) of compound 5n $\left(150 \mathrm{MHz}, \mathrm{CDCl}_{3}\right)$

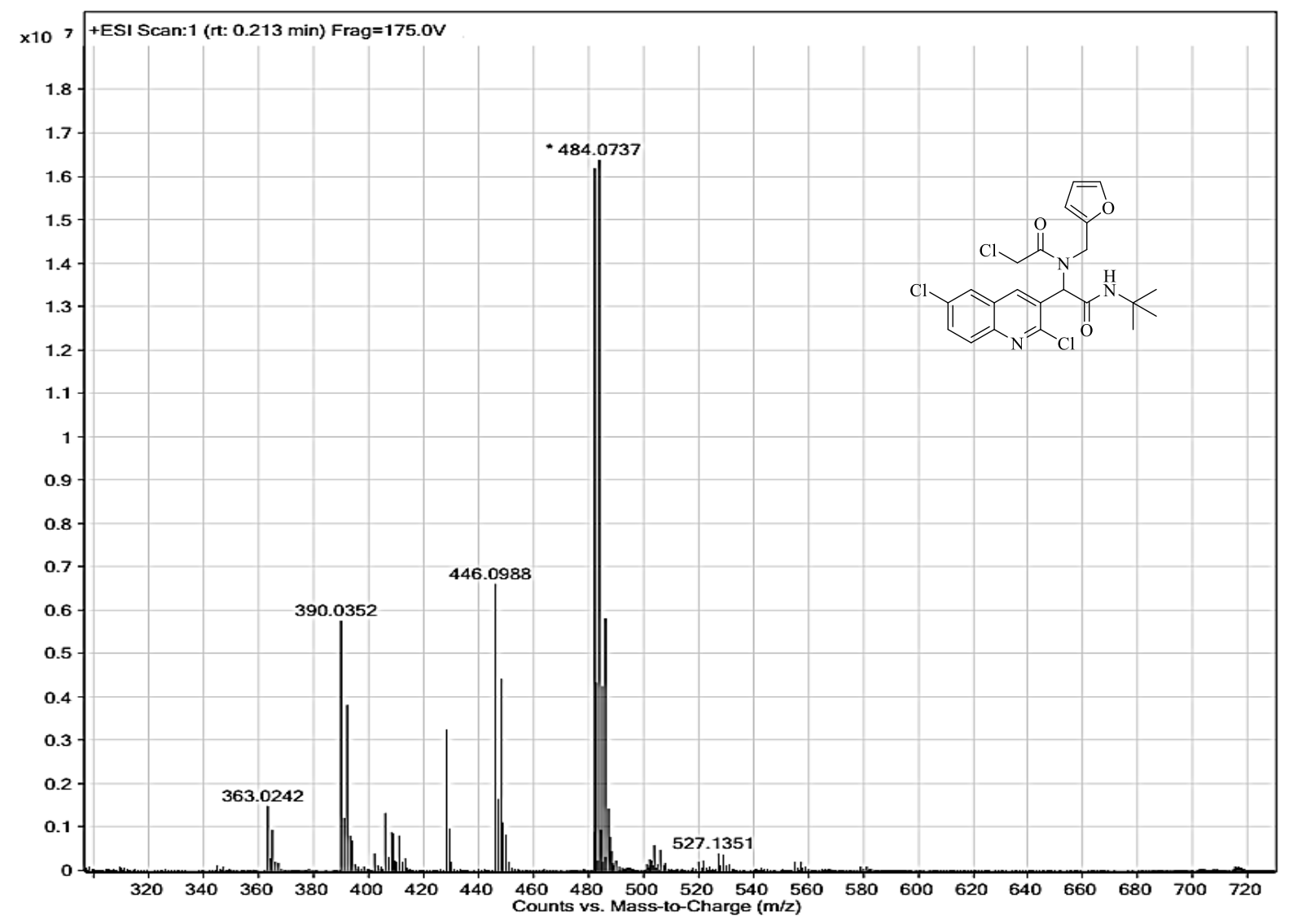

Figure S54: HRMS-ESI of $5 n$ with formula $\mathrm{C}_{22} \mathrm{H}_{22}{ }^{35} \mathrm{Cl}_{2}{ }^{37} \mathrm{ClN}_{3} \mathrm{O}_{3}$ and $[\mathrm{M}+\mathrm{H}]^{+} 484.0746$. 


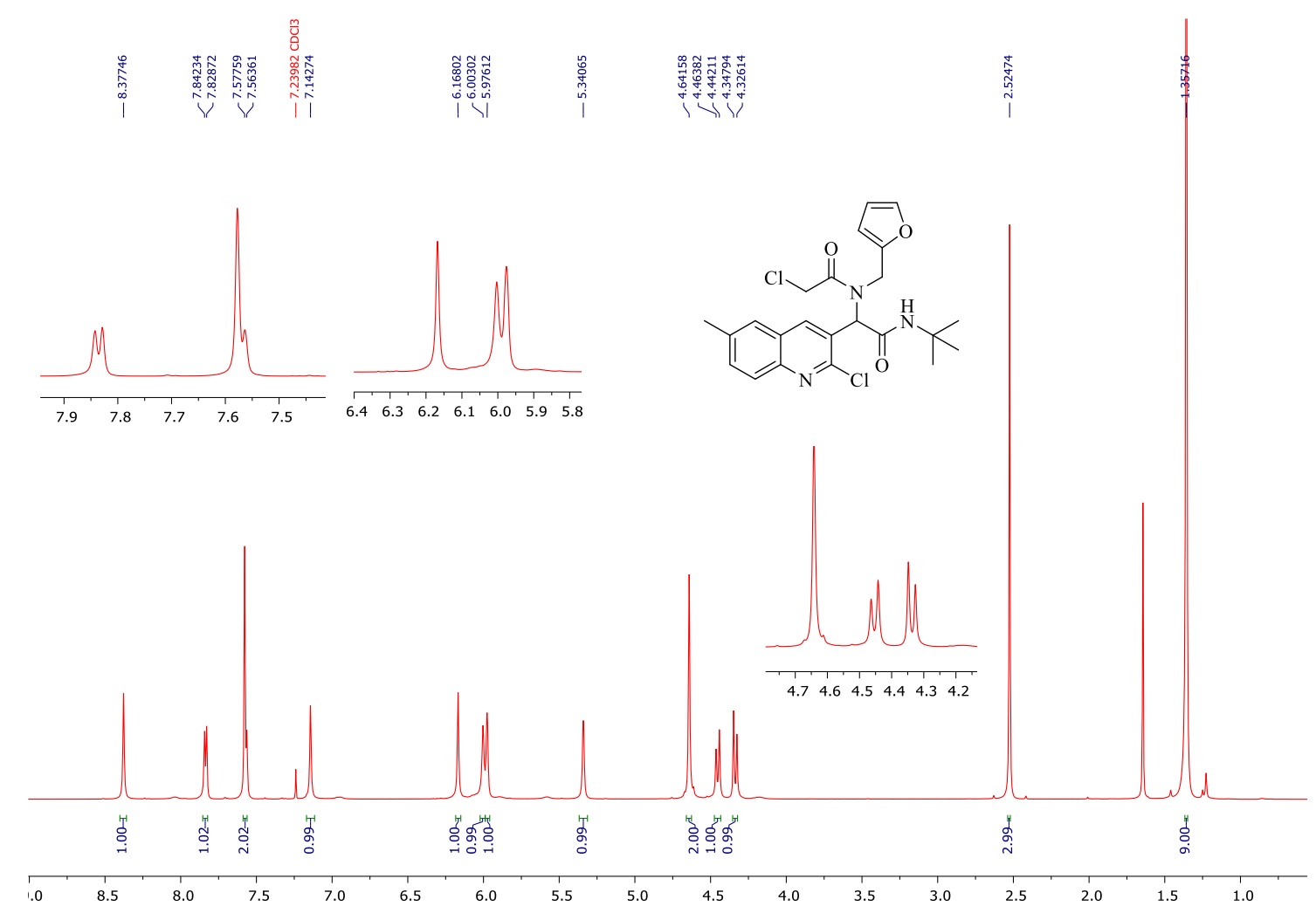

Figure S55: ${ }^{1} \mathrm{H}-\mathrm{NMR}$ of compound $\mathbf{5 0}\left(600 \mathrm{MHz}, \mathrm{CDCl}_{3}\right)$
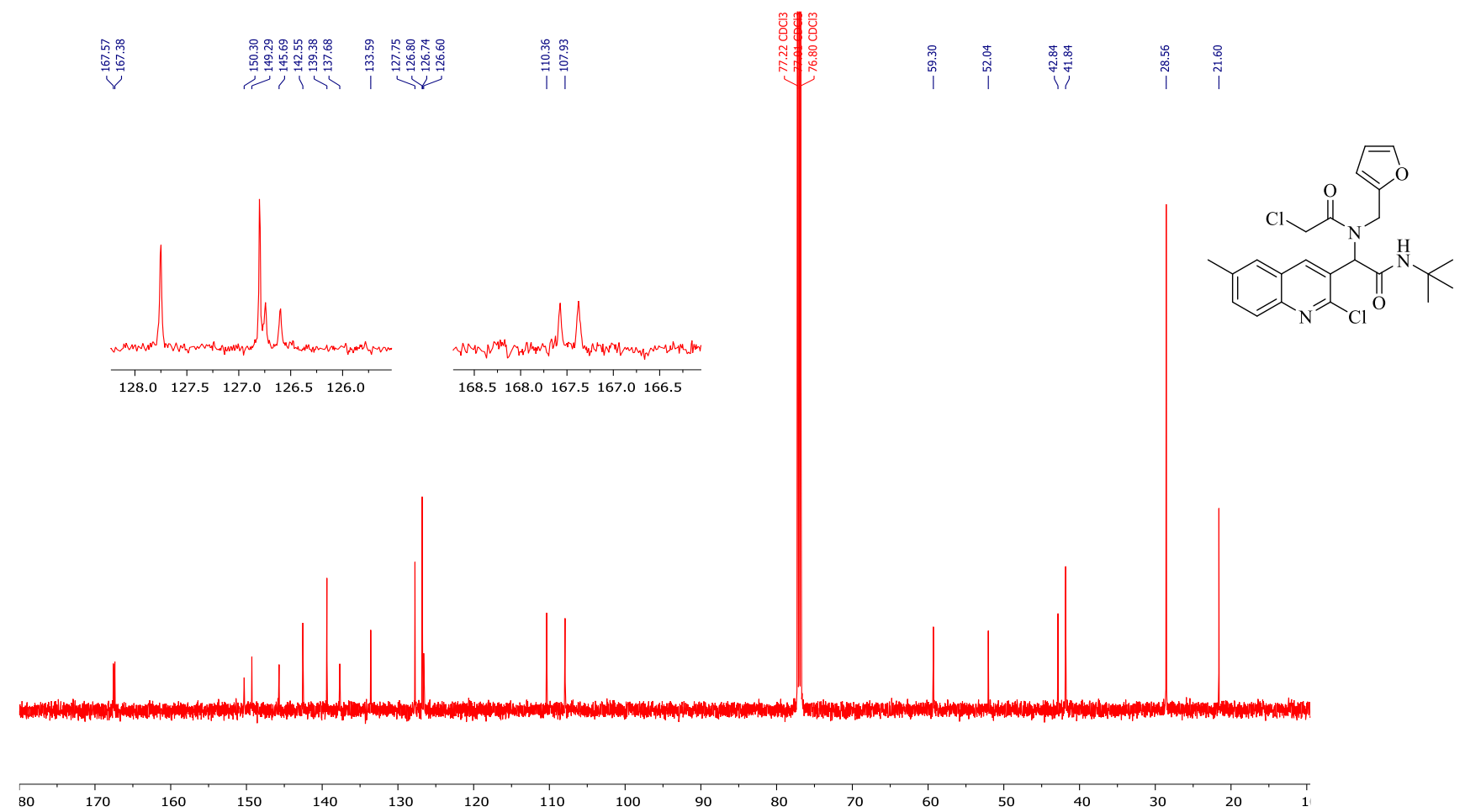

Figure S56: ${ }^{13} \mathrm{C}-\mathrm{NMR}$ of compound $\mathbf{5 o}\left(150 \mathrm{MHz}, \mathrm{CDCl}_{3}\right)$ 


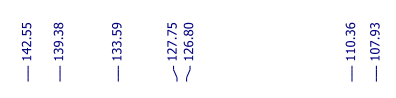

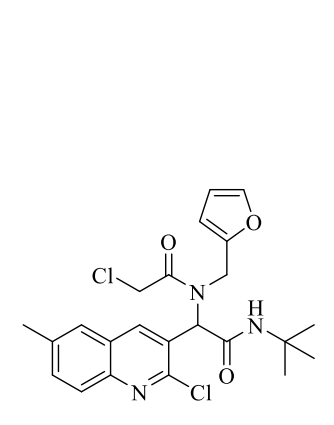

$\stackrel{\substack{0 \\ 0 \\ 0}}{\substack{0 \\ 1}}$

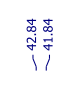
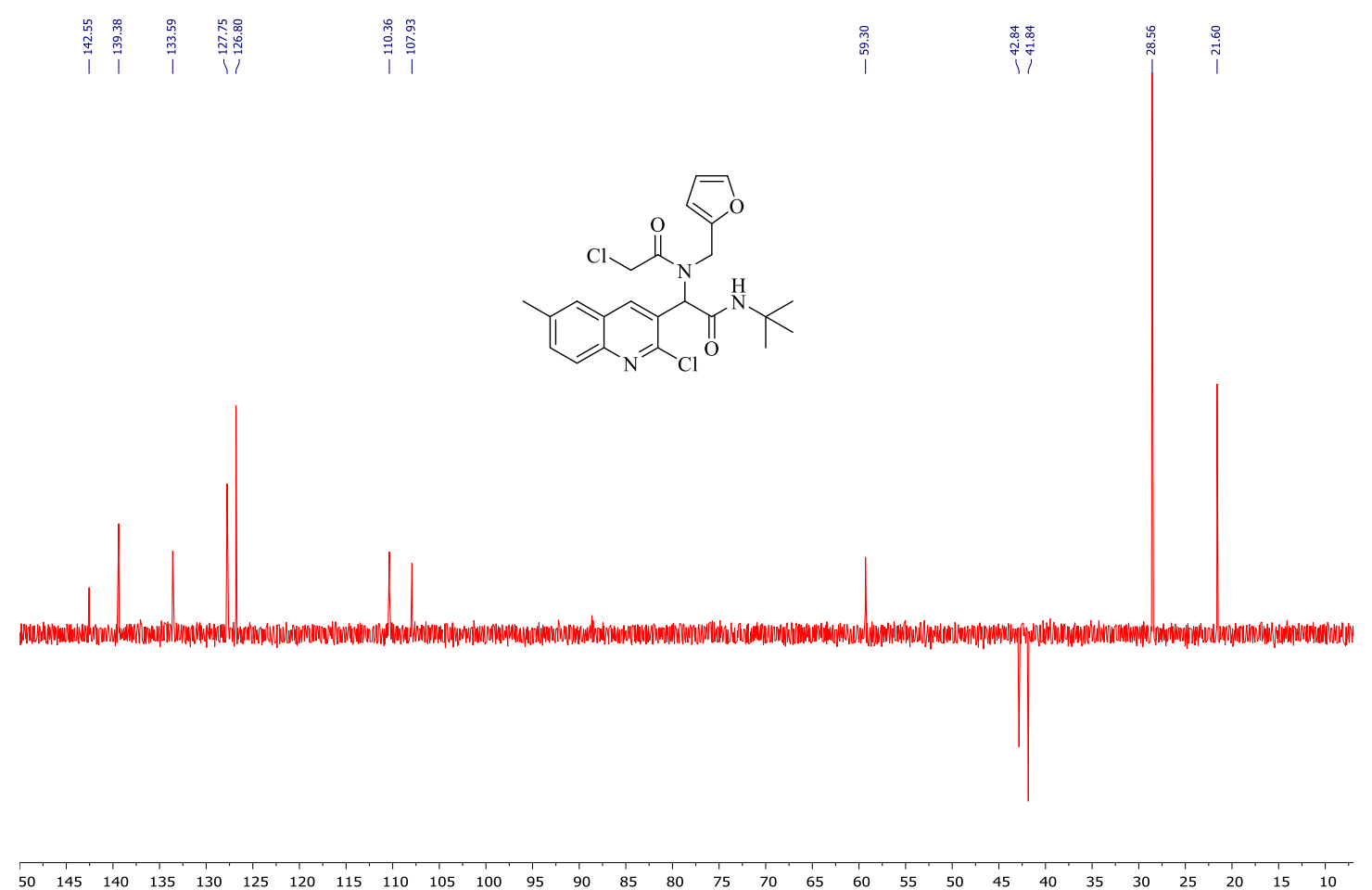

Figure S57: ${ }^{13} \mathrm{C}-\mathrm{NMR}$ (DEPT 135) of compound $\mathbf{5 o}\left(150 \mathrm{MHz}, \mathrm{CDCl}_{3}\right)$

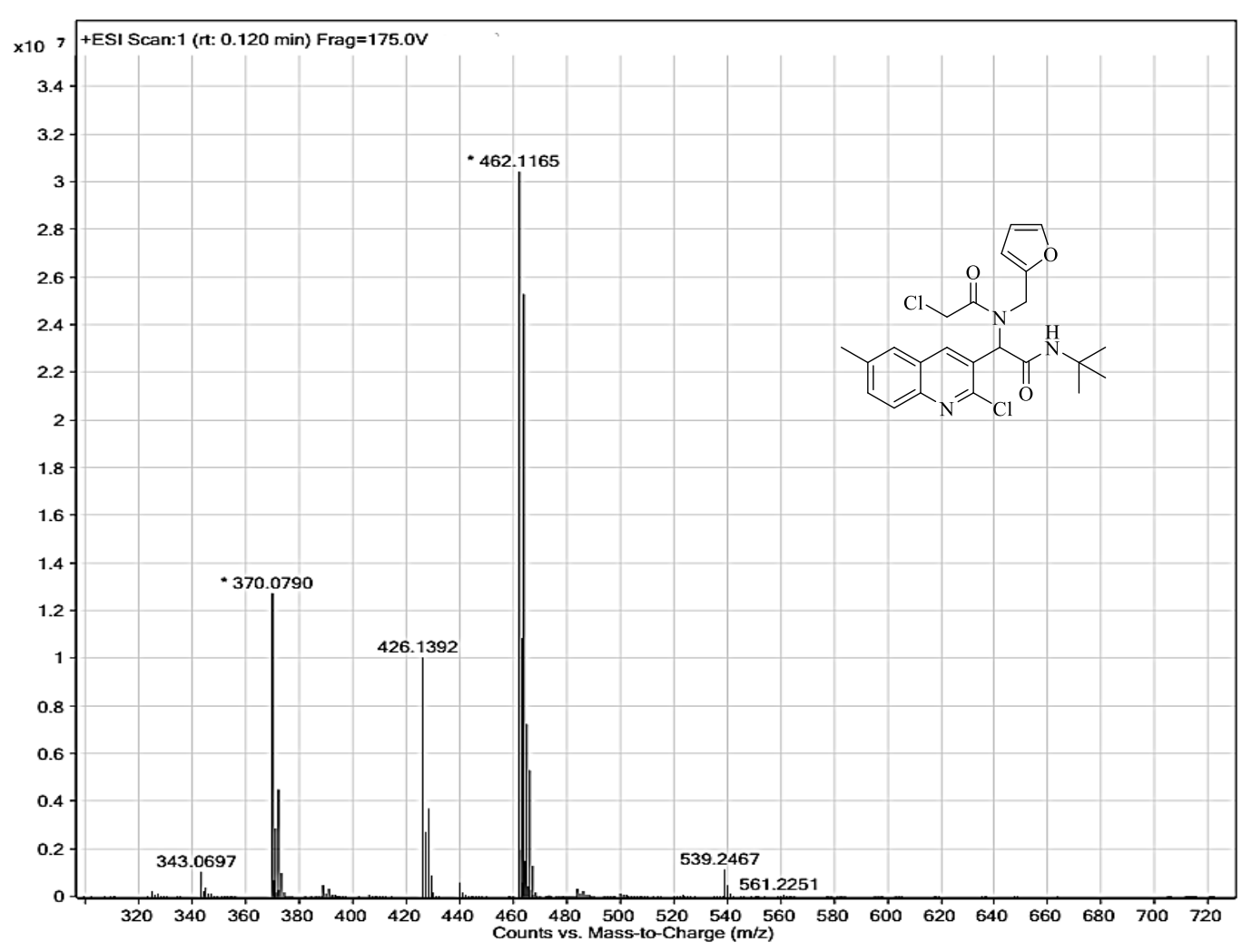

Figure S58: HRMS-ESI of $\mathbf{5 o}$ with formula $\mathrm{C}_{23} \mathrm{H}_{25} \mathrm{Cl}_{2} \mathrm{~N}_{3} \mathrm{O}_{3}$ and $[\mathrm{M}+\mathrm{H}]^{+} 462.1173$. 


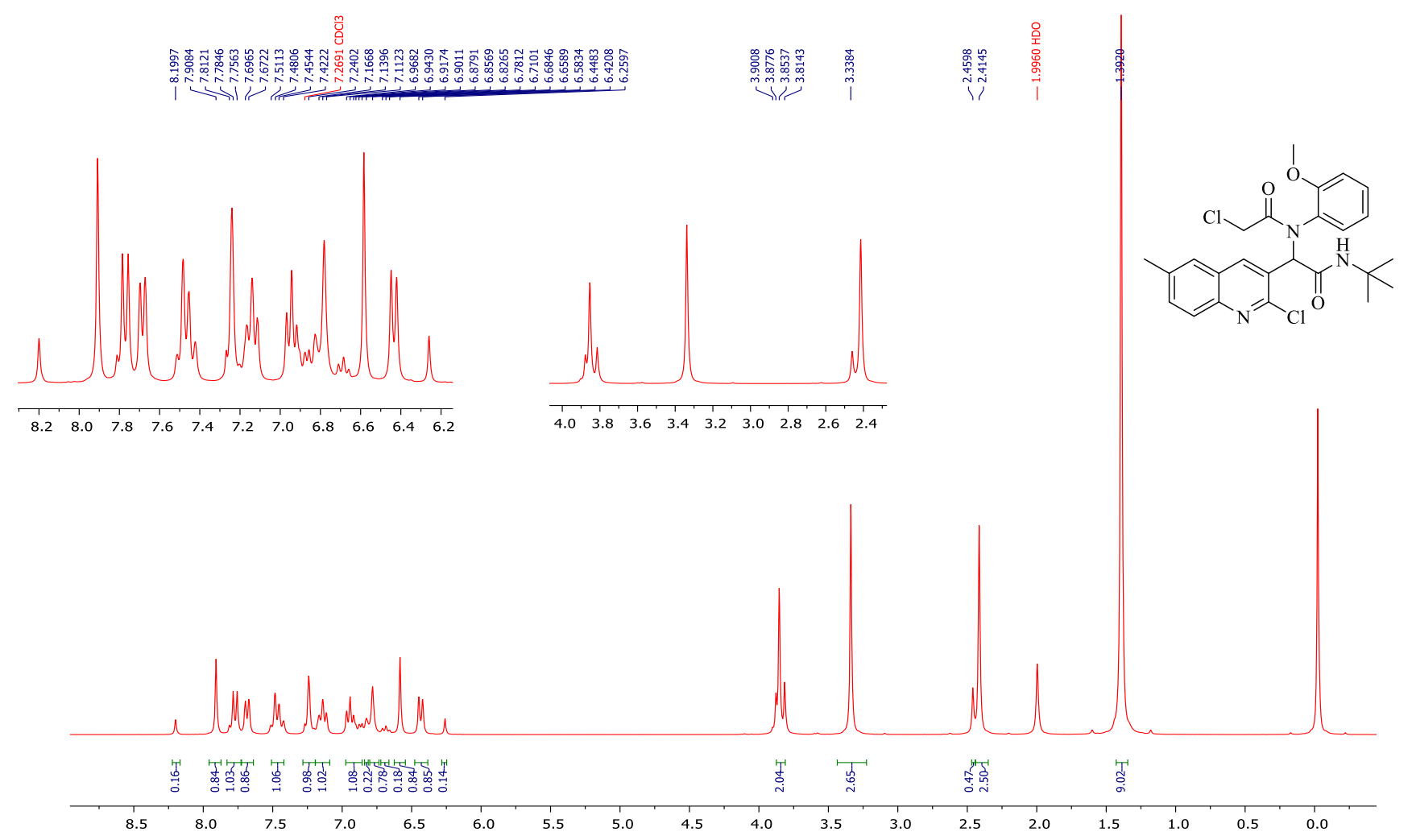

Figure S59: ${ }^{1} \mathrm{H}-\mathrm{NMR}$ of compound $\mathbf{5 p}\left(300 \mathrm{MHz}, \mathrm{CDCl}_{3}\right)$
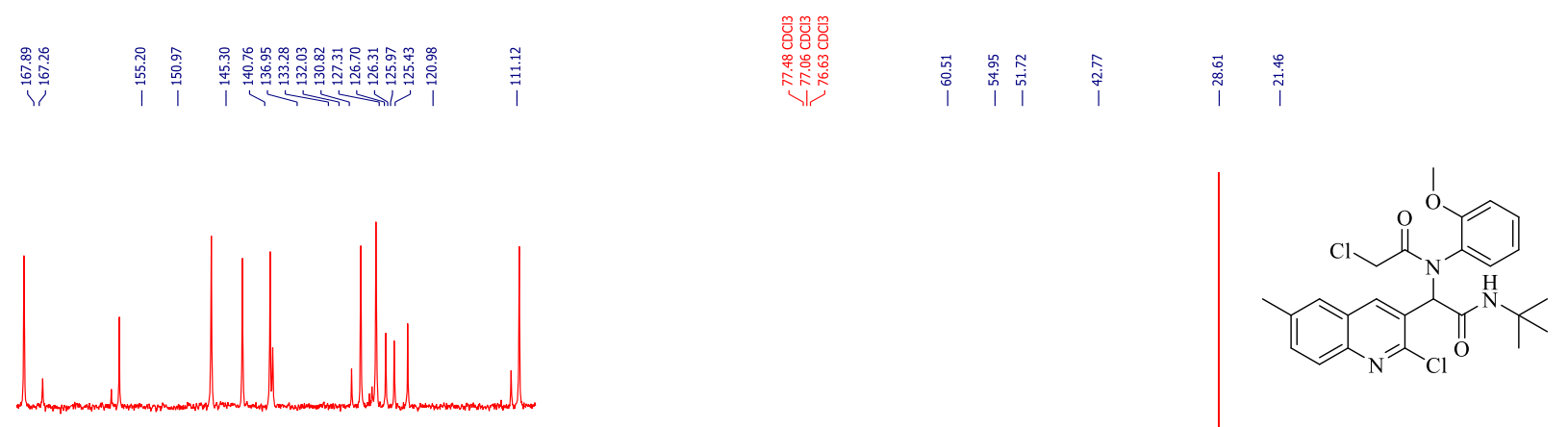

$\begin{array}{llllllllll}140 & 138 & 136 & 134 & 132 & 130 & 128 & 126 & 124 & 122\end{array}$
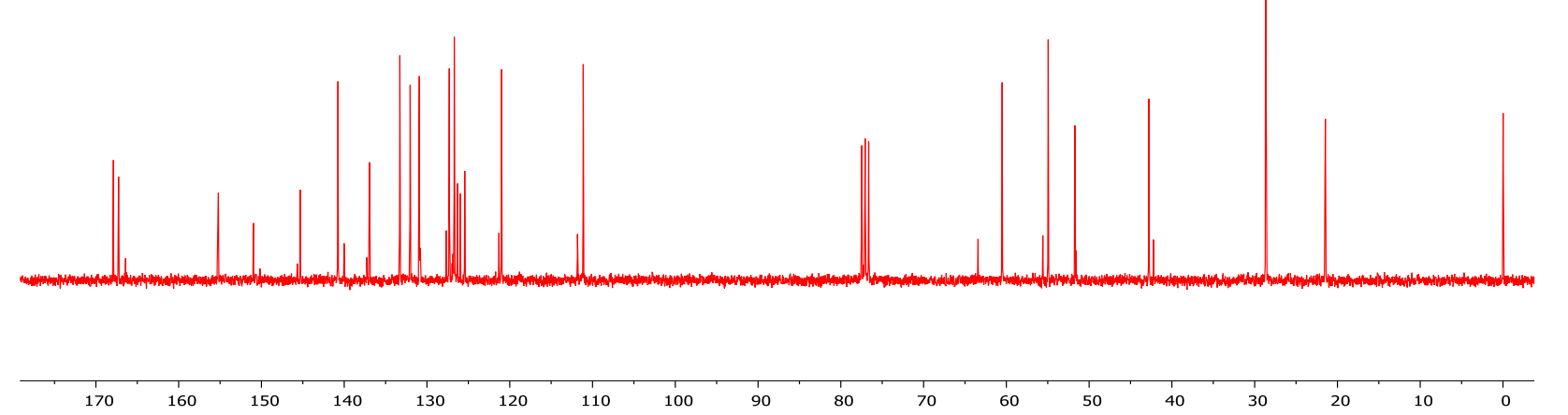

Figure S60: ${ }^{13} \mathrm{C}-\mathrm{NMR}$ of compound $\mathbf{5 p}\left(75 \mathrm{MHz}, \mathrm{CDCl}_{3}\right)$ 


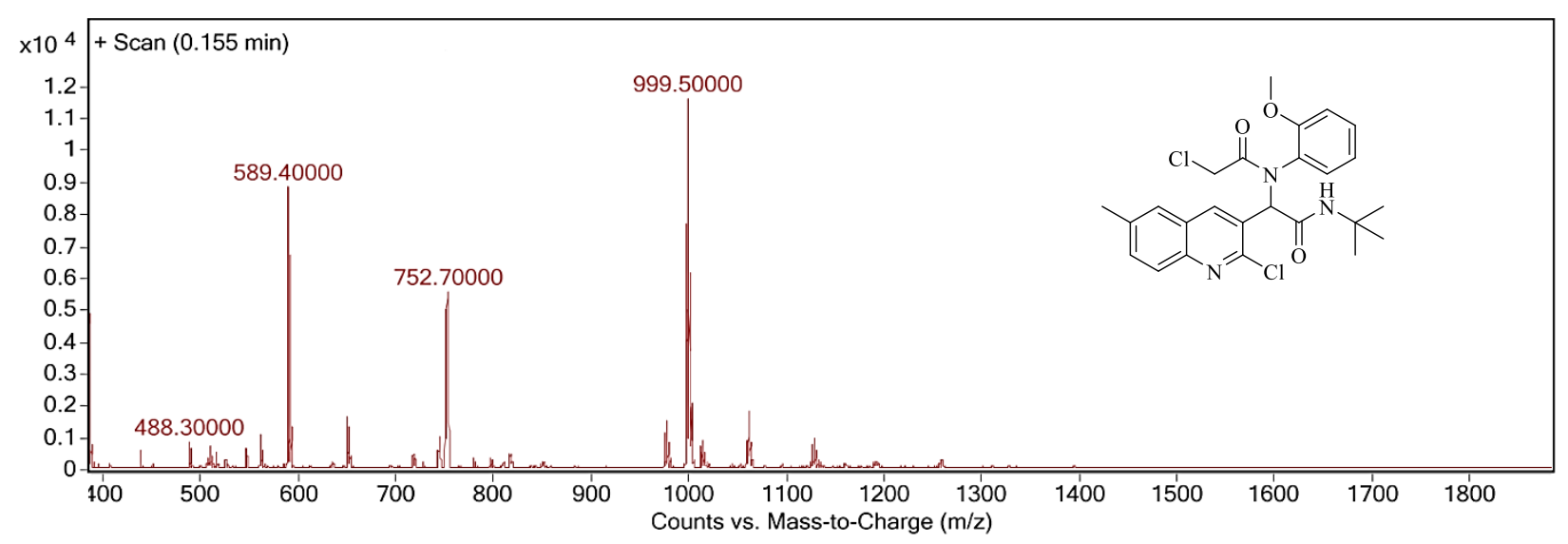

Figure S61: ESI-MS (+) of compound 5p with formula $\mathrm{C}_{25} \mathrm{H}_{27} \mathrm{Cl}_{2} \mathrm{~N}_{3} \mathrm{O}_{3}$ and $[\mathrm{M}+\mathrm{H}]^{+} 488.3$. 


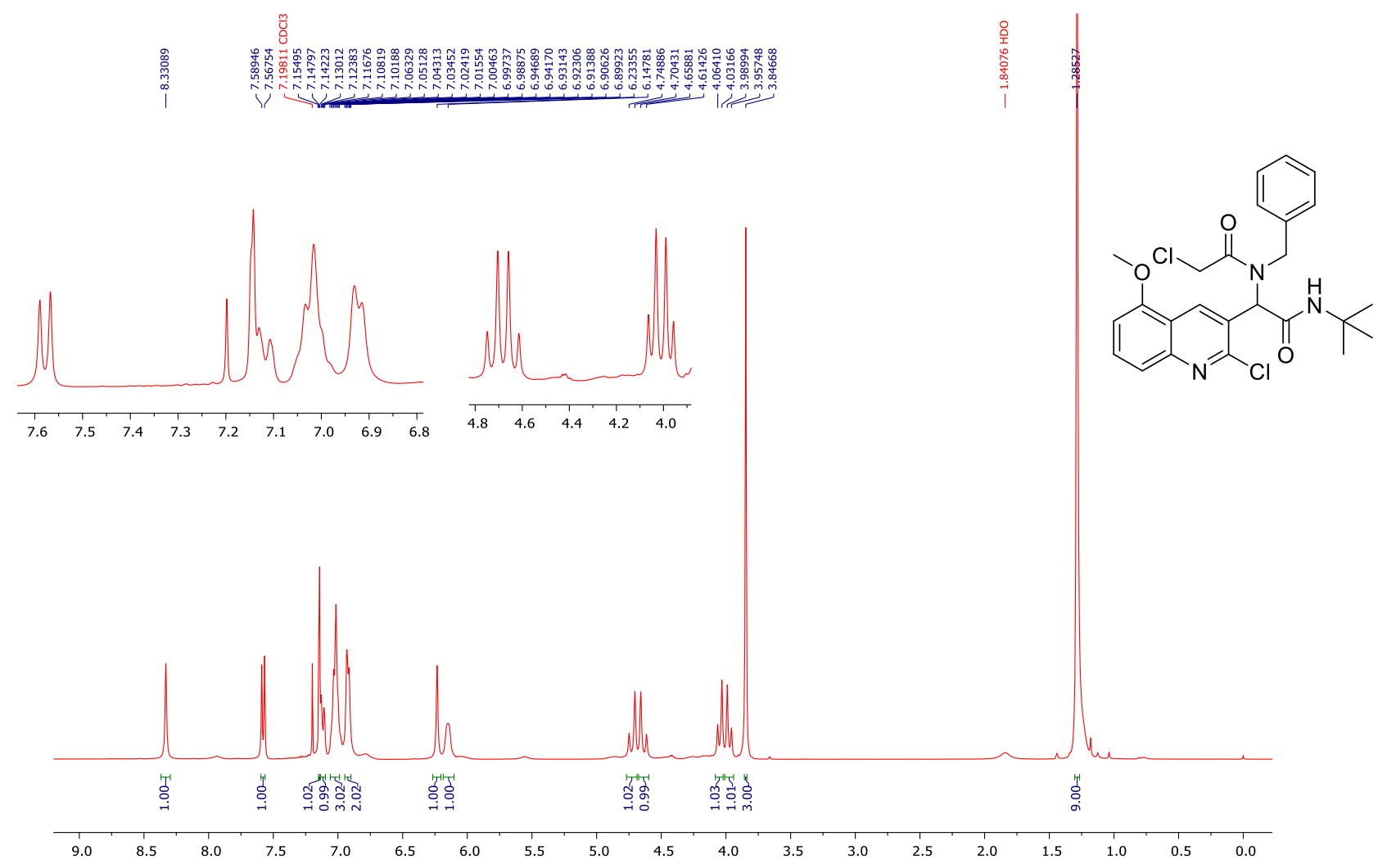

Figure S62: ${ }^{1} \mathrm{H}-\mathrm{NMR}$ of compound $\mathbf{5 q}\left(400 \mathrm{MHz}, \mathrm{CDCl}_{3}\right)$

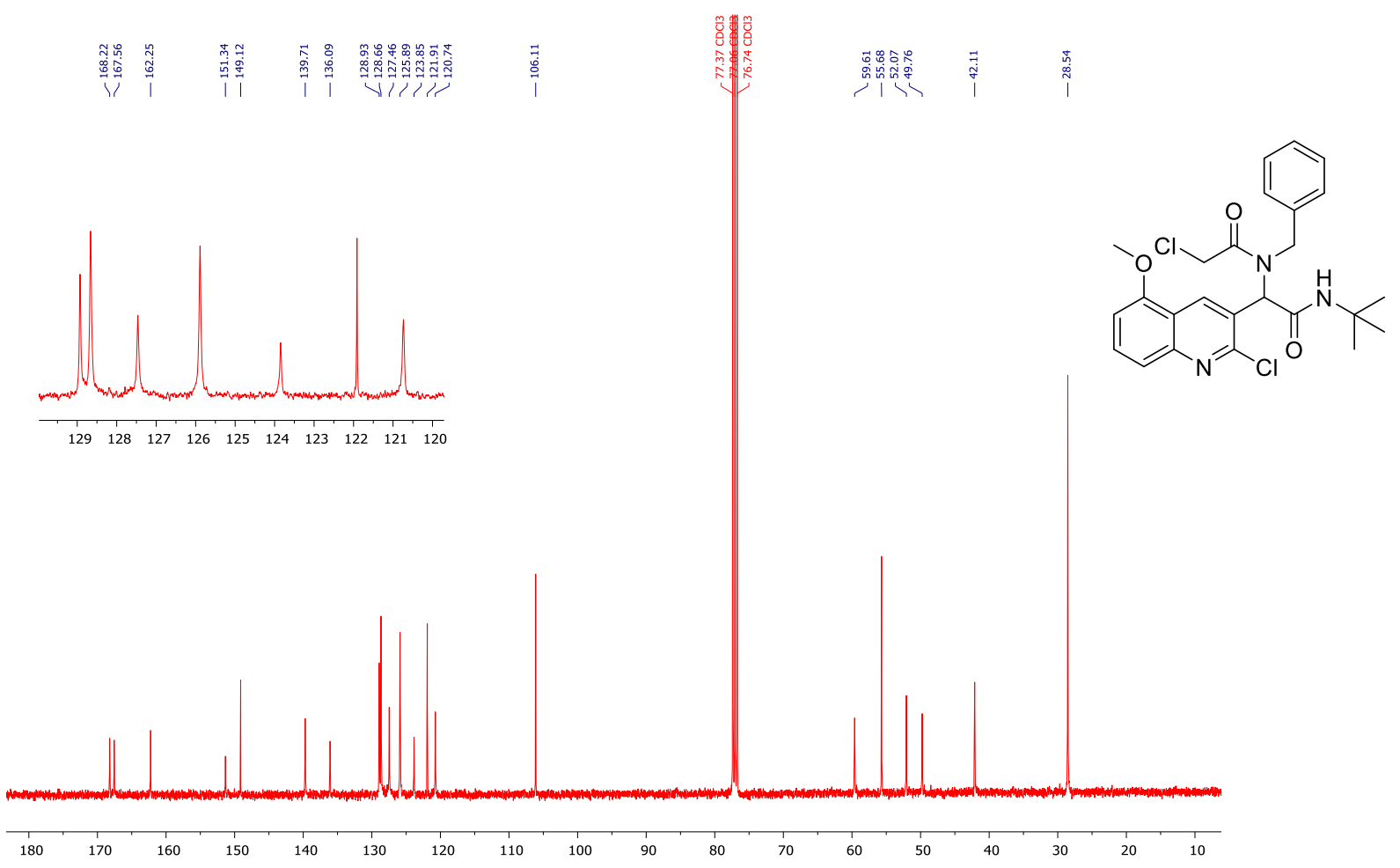

Figure S63: ${ }^{13} \mathrm{C}-\mathrm{NMR}$ of compound $\mathbf{5 q}\left(100 \mathrm{MHz}, \mathrm{CDCl}_{3}\right)$ 


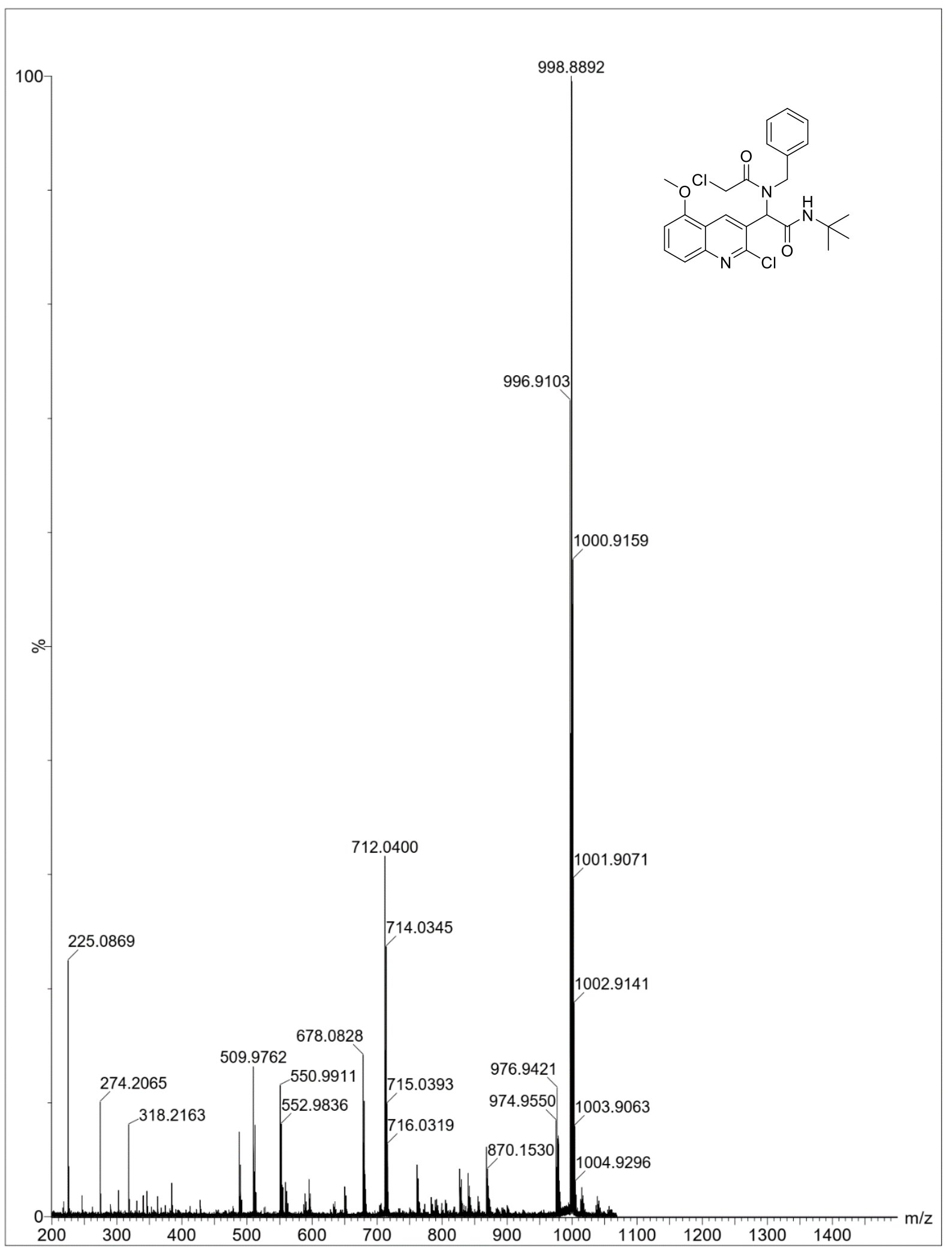

Figure S64: ESI-MS (+) of compound $5 q$ with formula $\mathrm{C}_{25} \mathrm{H}_{27} \mathrm{Cl}_{2} \mathrm{~N}_{3} \mathrm{NaO}_{3}[\mathrm{M}+\mathrm{Na}]^{+} 509.9862$ 


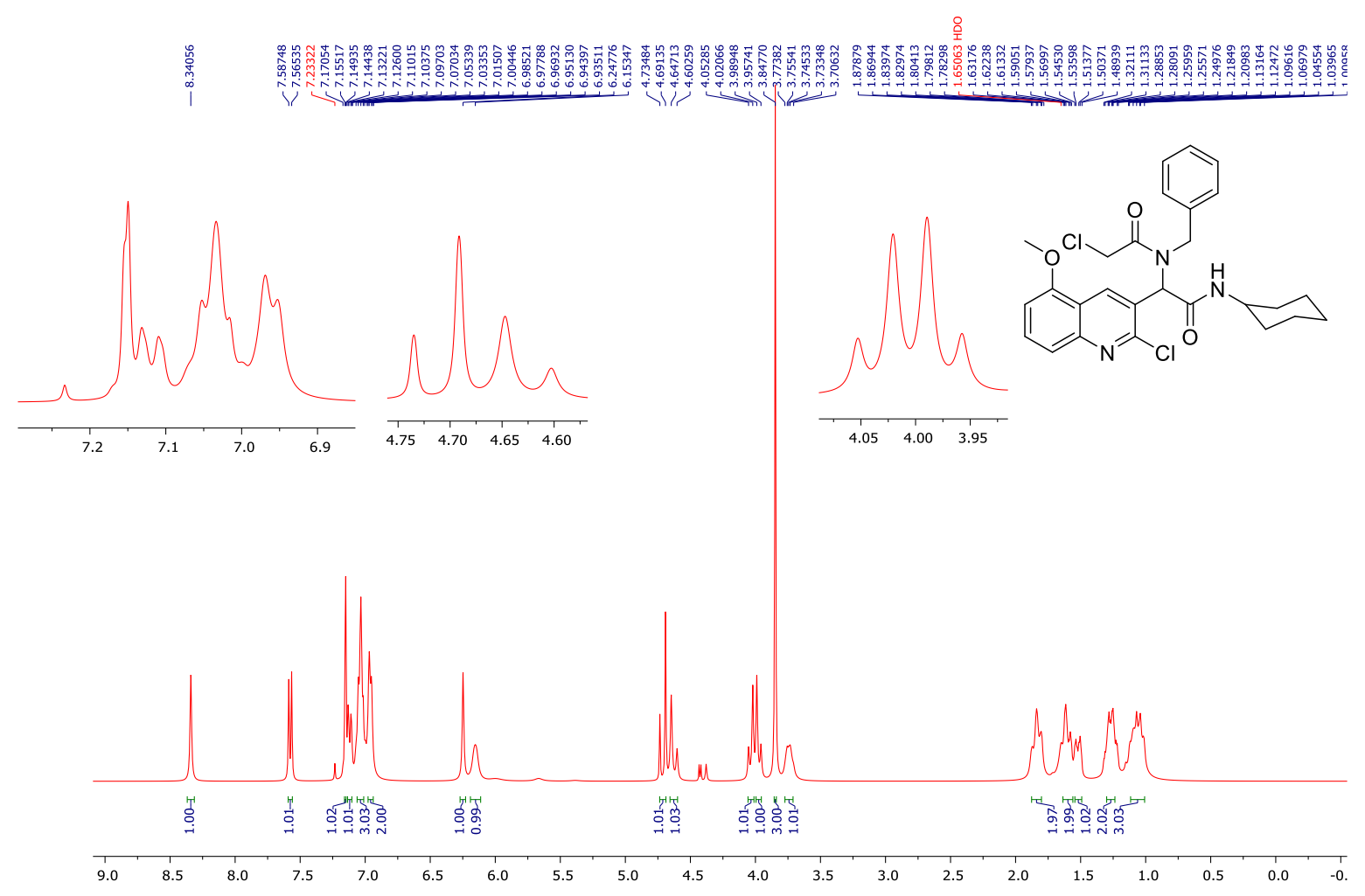

Figure S65: ${ }^{1} \mathrm{H}-\mathrm{NMR}$ of compound $\mathbf{5 r}\left(400 \mathrm{MHz}, \mathrm{CDCl}_{3}\right)$

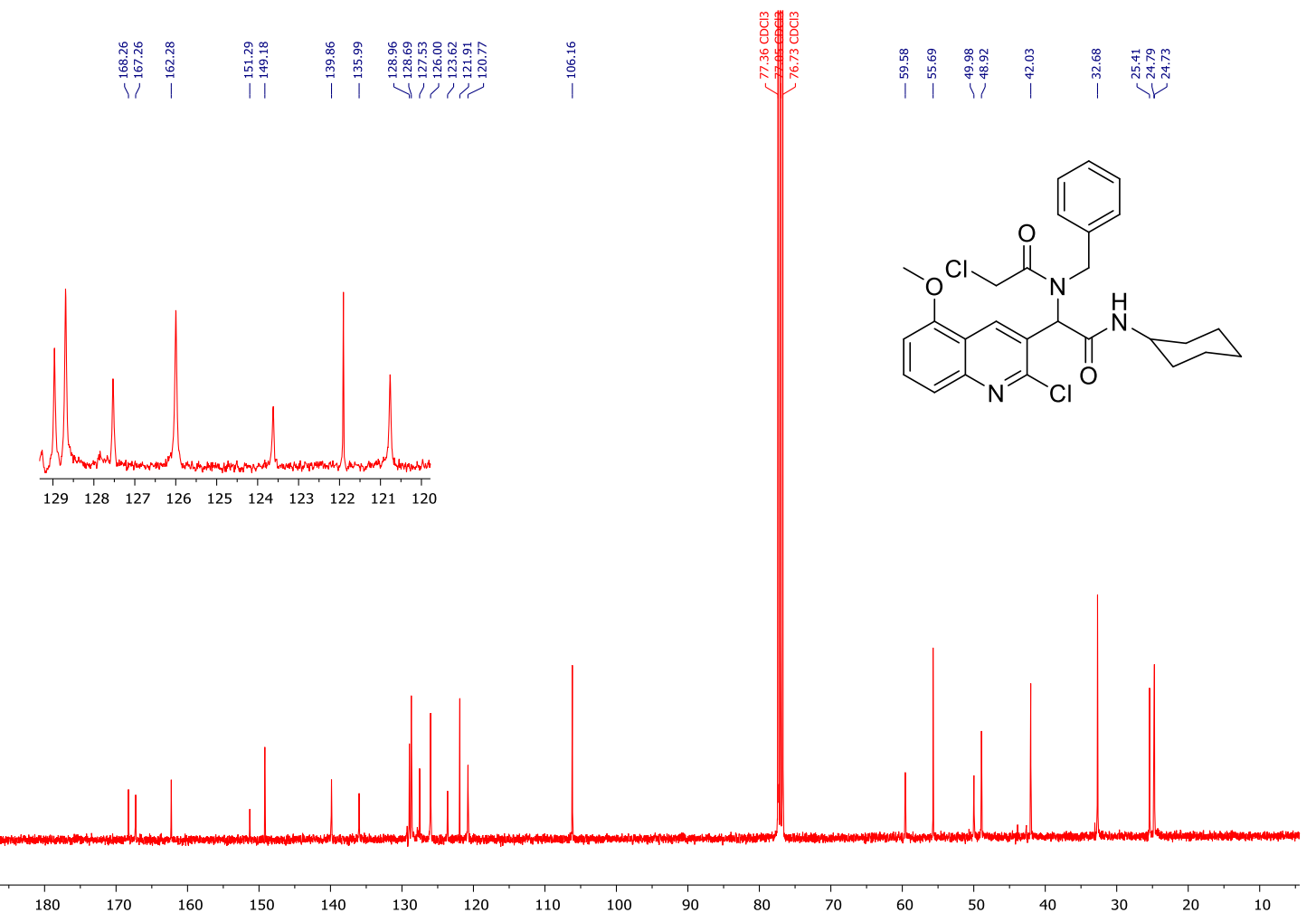

Figure S66: ${ }^{13} \mathrm{C}-\mathrm{NMR}$ of compound $\mathbf{5 r}\left(100 \mathrm{MHz}, \mathrm{CDCl}_{3}\right)$ 


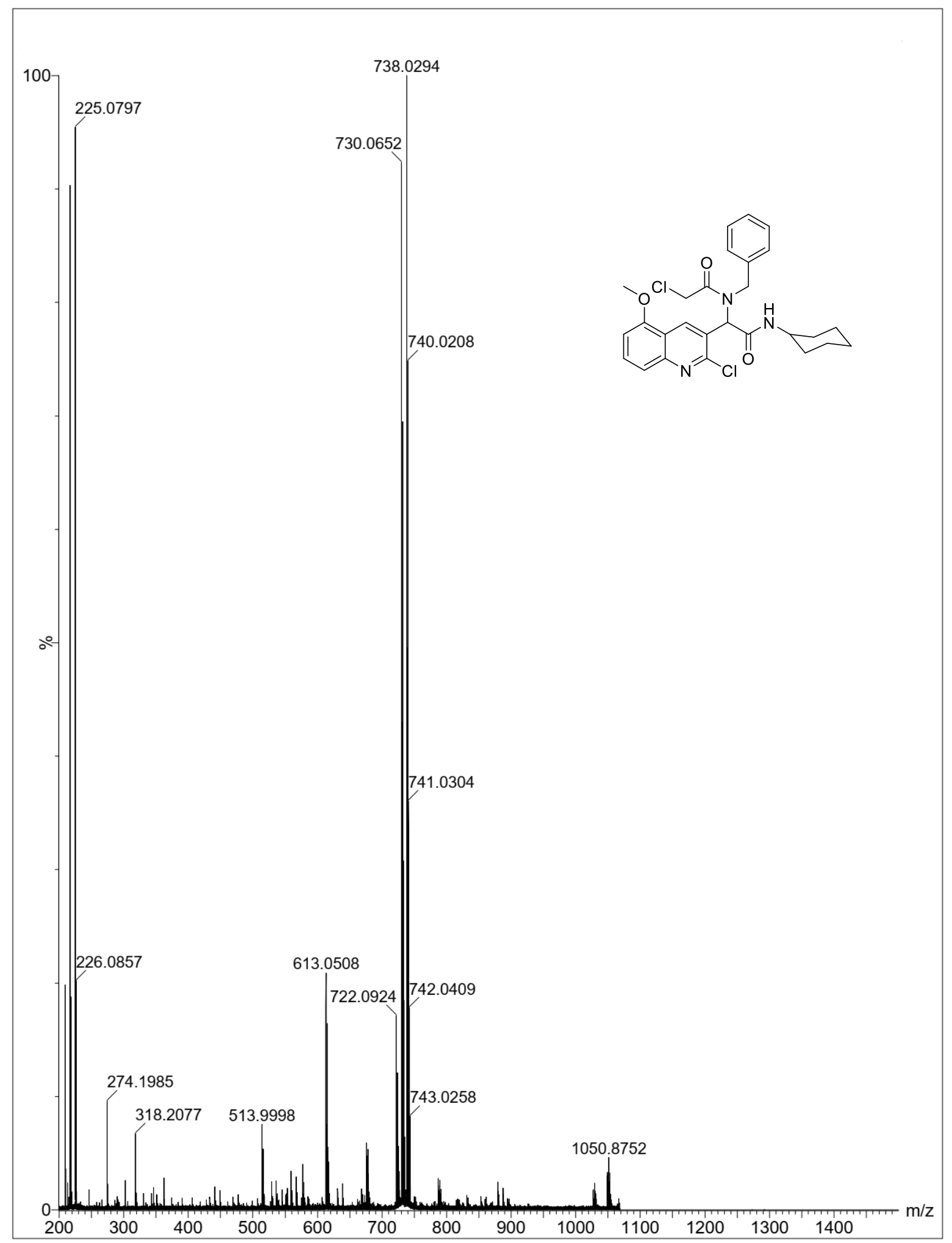

Figure S67: ESI-MS (+) of compound $\mathbf{5 r}$ with formula $\mathrm{C}_{27} \mathrm{H}_{29} \mathrm{Cl}_{2} \mathrm{~N}_{3} \mathrm{O}_{3}$ and $[\mathrm{M}+\mathrm{H}]^{+} 513.9998$. 

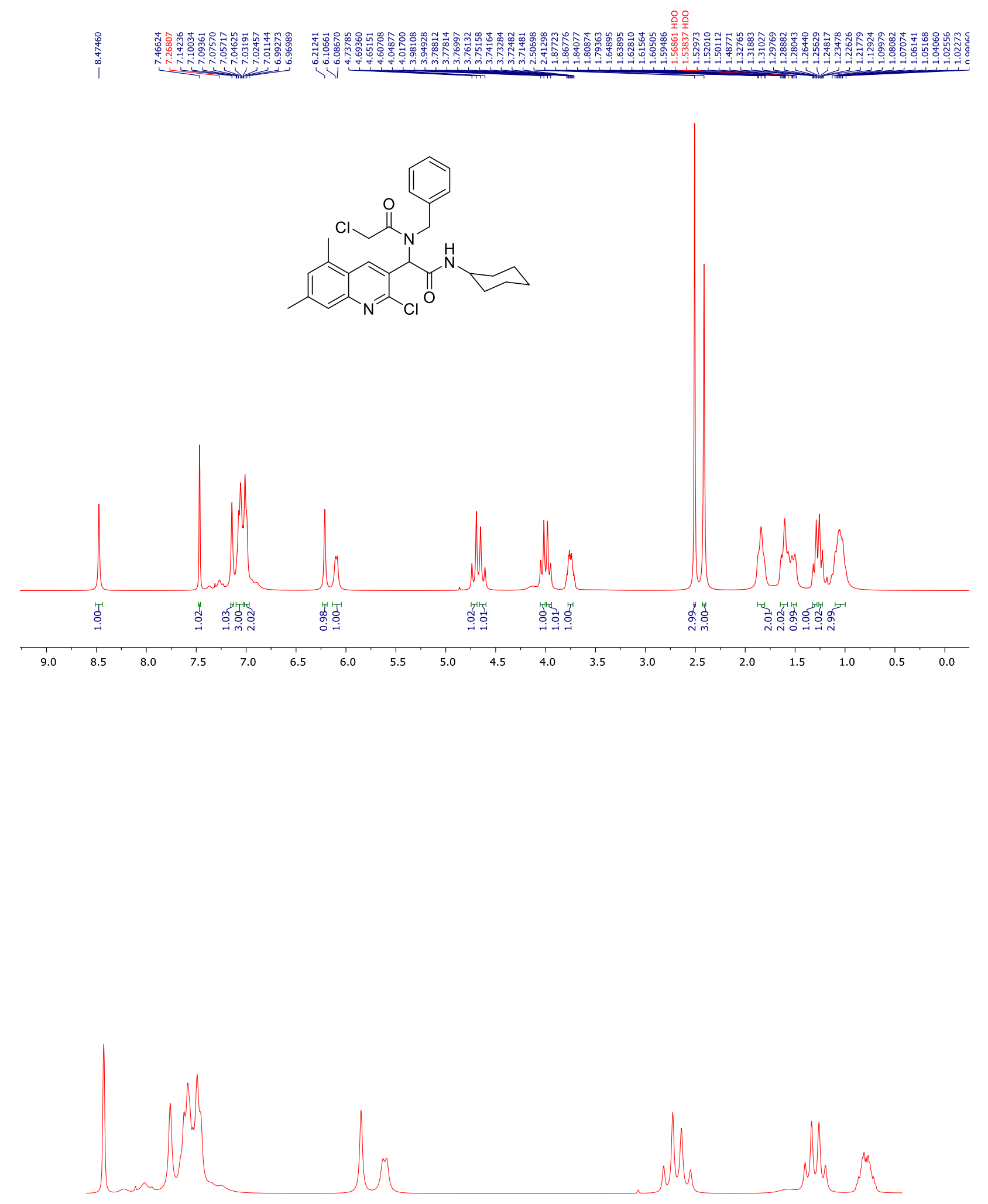

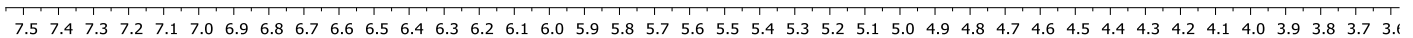

Figure S68: ${ }^{1} \mathrm{H}-\mathrm{NMR}$ of compound $5 \mathrm{~s}\left(400 \mathrm{MHz}, \mathrm{CDCl}_{3}\right)$ 


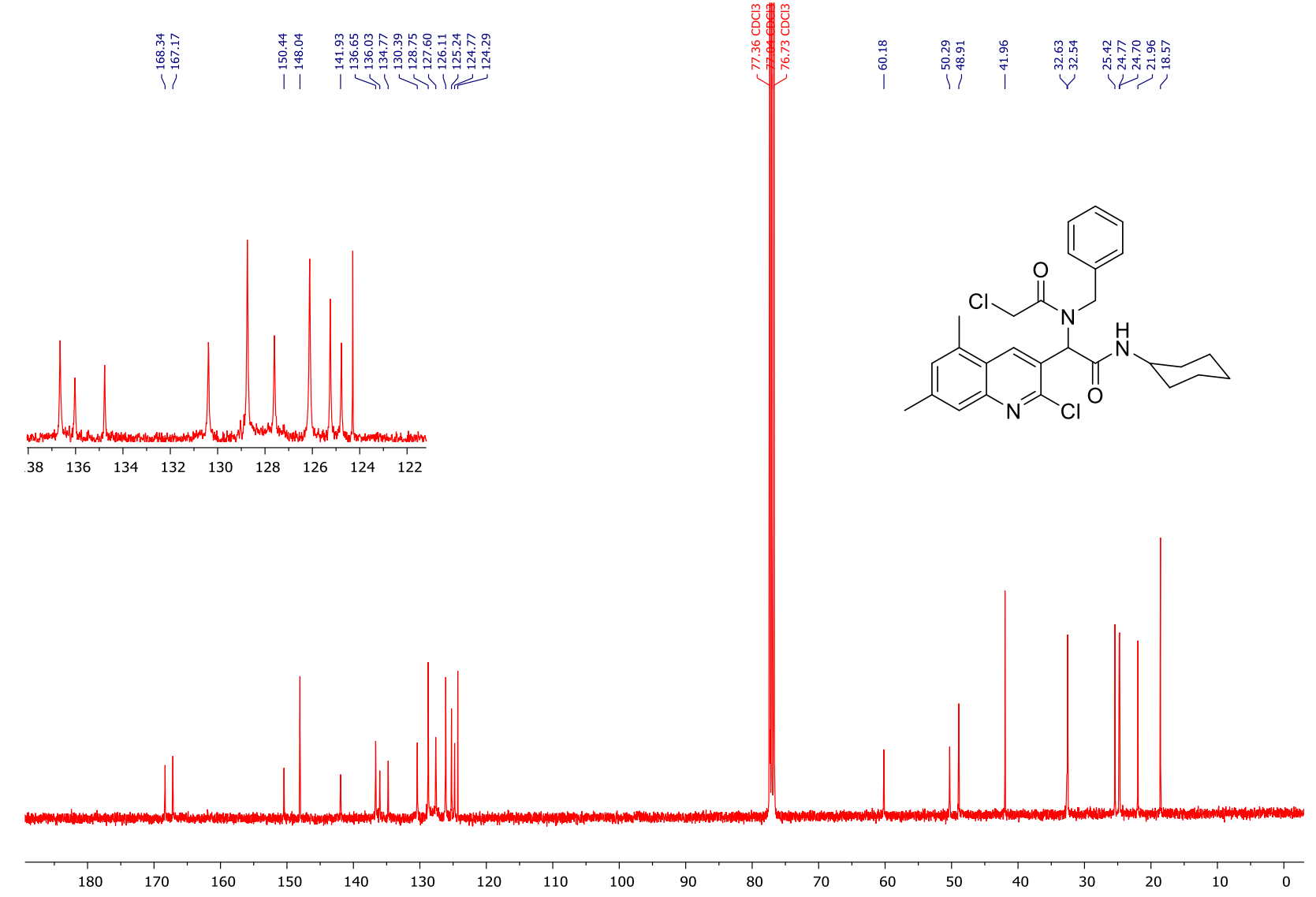

Figure S69: ${ }^{13} \mathrm{C}-\mathrm{NMR}$ of compound $\mathbf{5 s}\left(100 \mathrm{MHz}, \mathrm{CDCl}_{3}\right)$ 


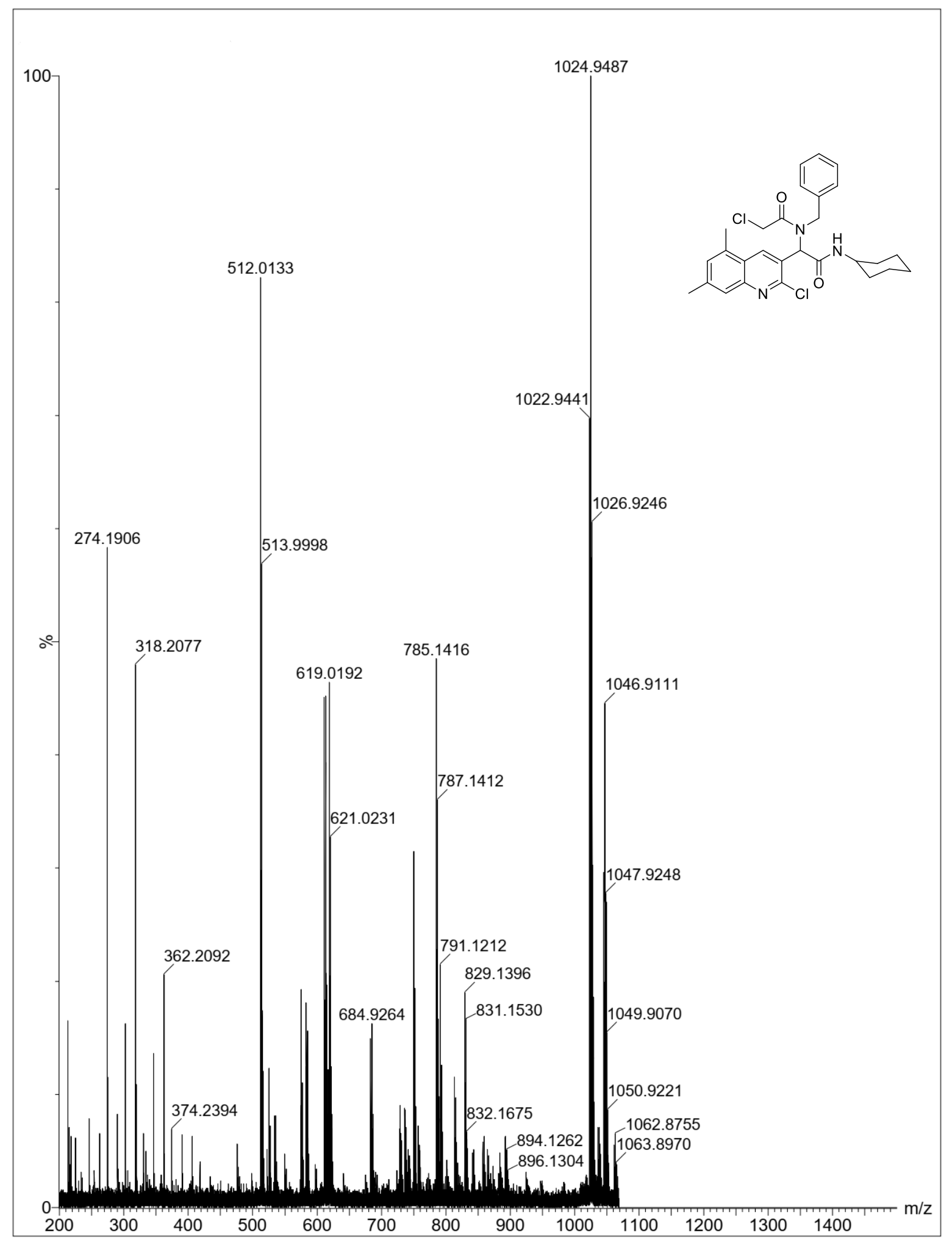

Figure S70: ESI-MS (+) of compound 5 s with formula $\mathrm{C}_{28} \mathrm{H}_{31} \mathrm{Cl}_{2} \mathrm{~N}_{3} \mathrm{O}_{2}$ and $[\mathrm{M}+\mathrm{H}]^{+} 512.0133$. 


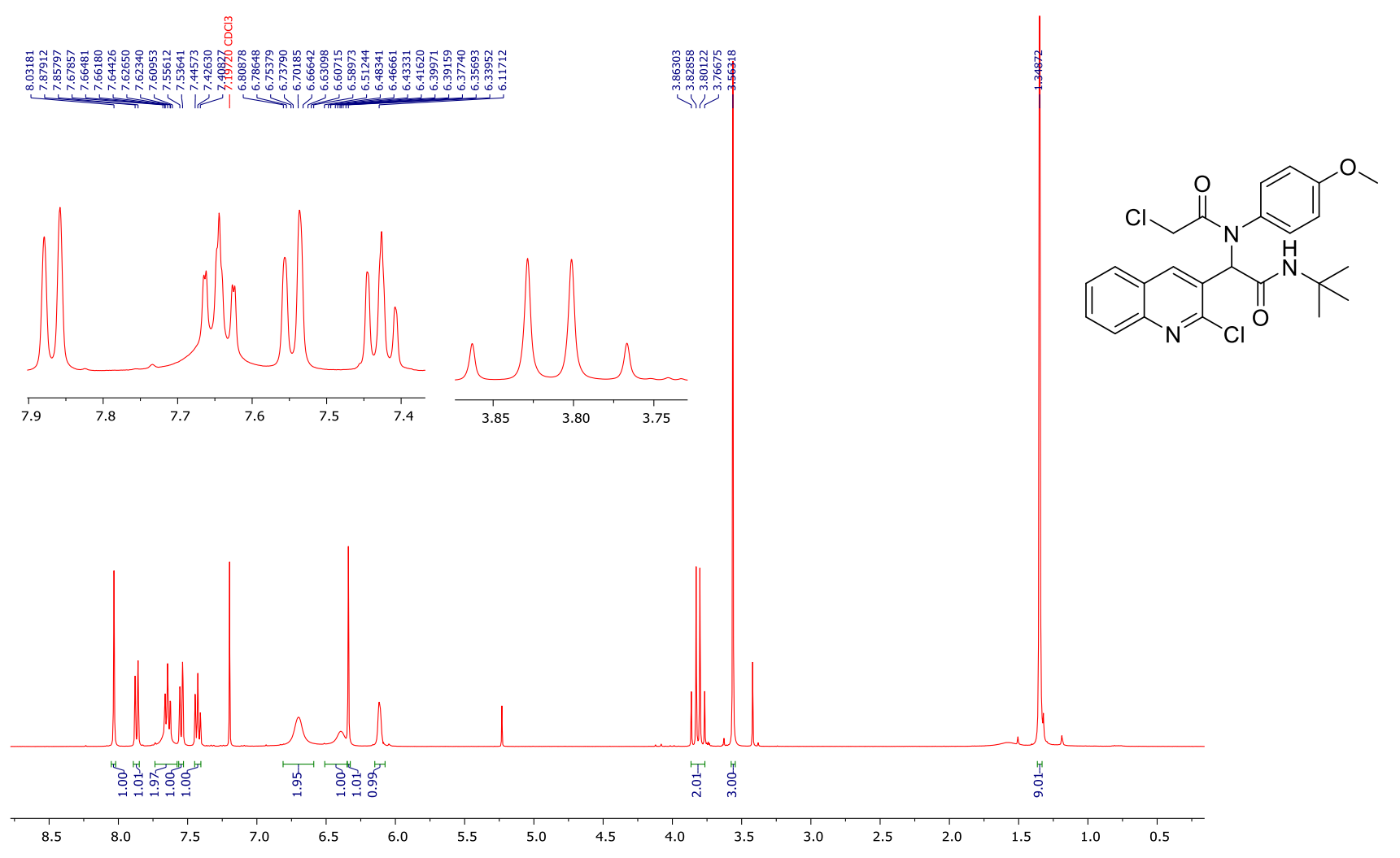

Figure S71: ${ }^{1} \mathrm{H}-\mathrm{NMR}$ of compound $\mathbf{5 t}\left(400 \mathrm{MHz}, \mathrm{CDCl}_{3}\right)$

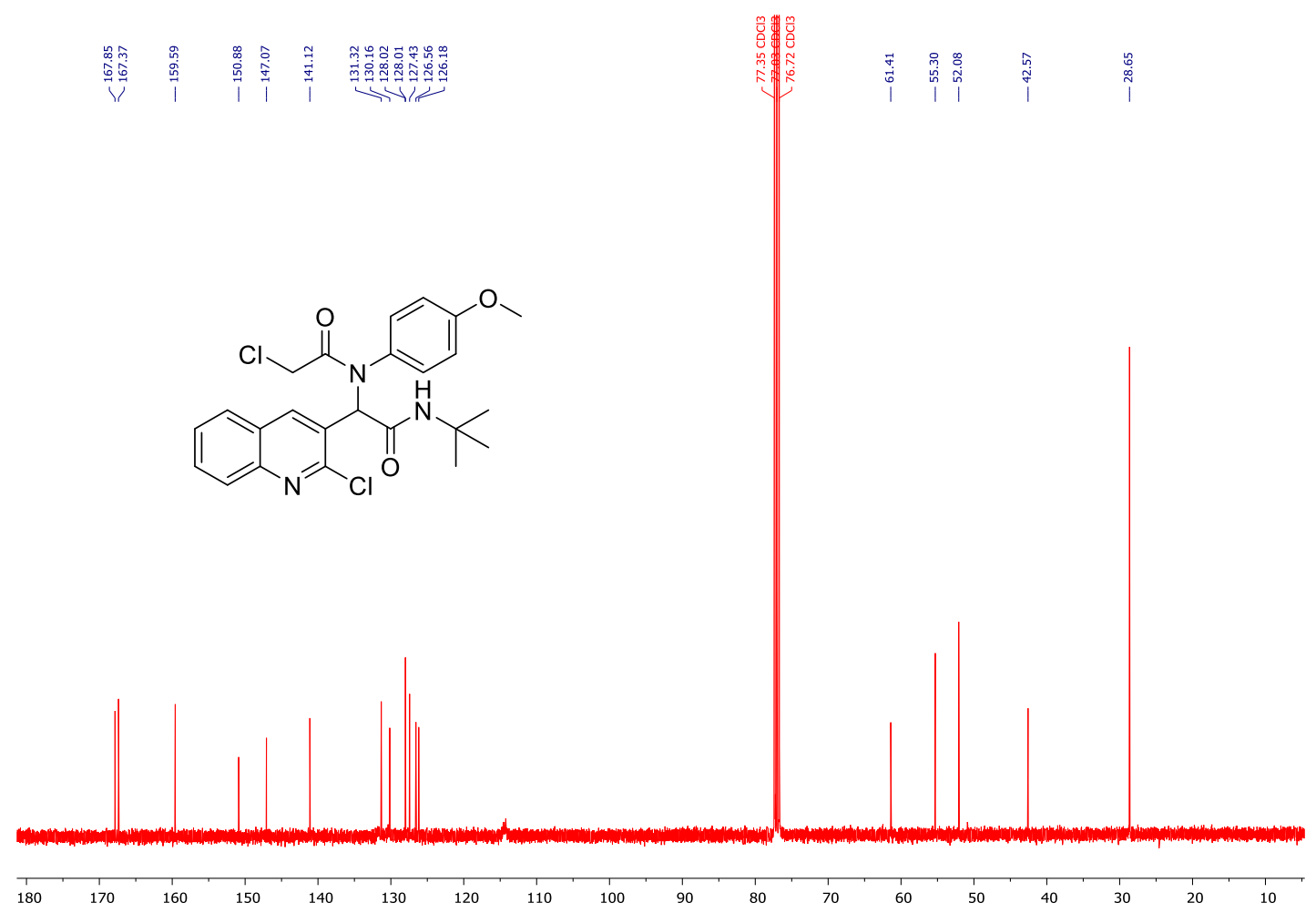

Figure S72: ${ }^{13} \mathrm{C}-\mathrm{NMR}$ of compound $\mathbf{5 t}\left(100 \mathrm{MHz}, \mathrm{CDCl}_{3}\right)$ 


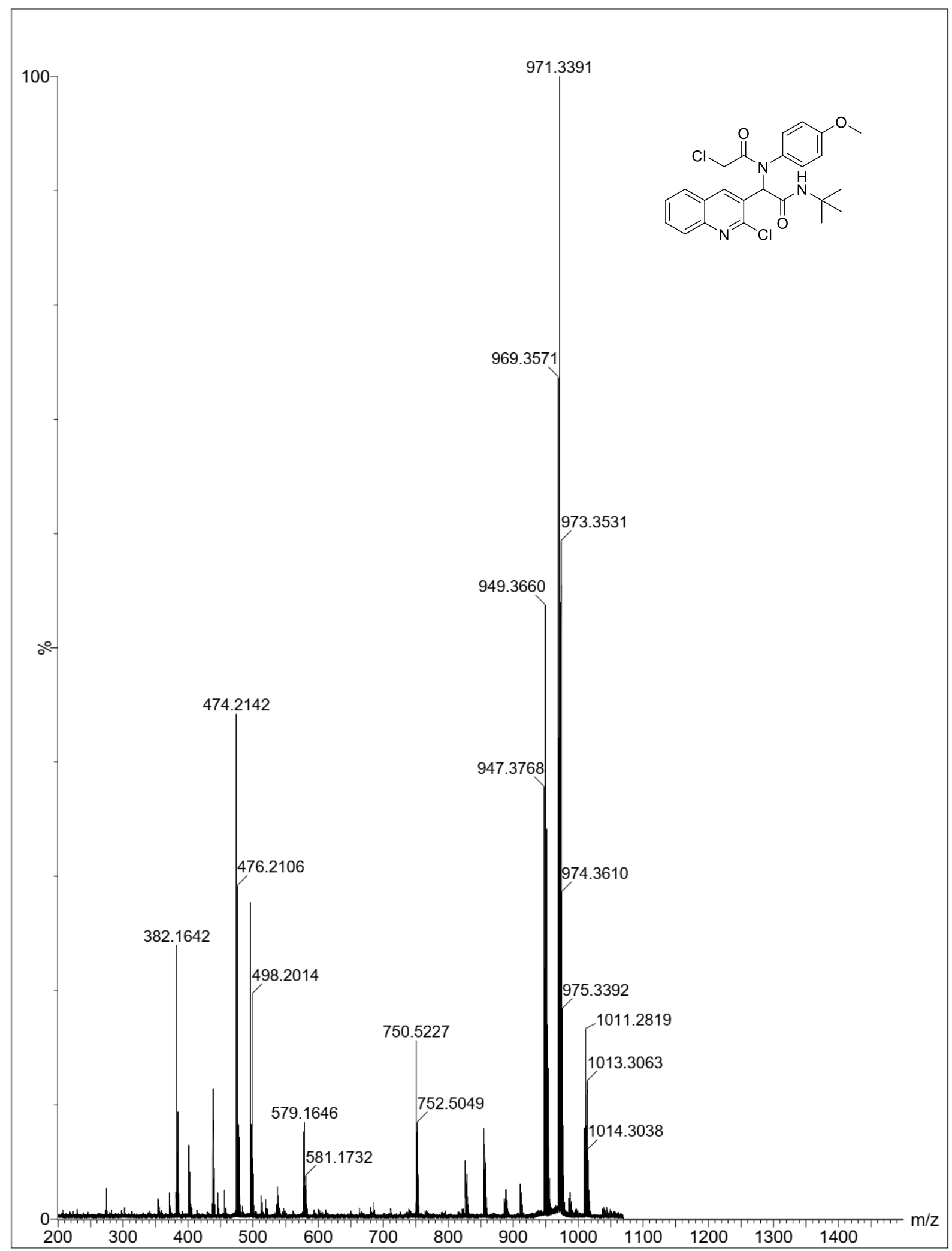

Figure S73: ESI-MS (+) of compound $\mathbf{5 t}$ with formula $\mathrm{C}_{24} \mathrm{H}_{25} \mathrm{Cl}_{2} \mathrm{~N}_{3} \mathrm{O}_{3}$ and $[\mathrm{M}+\mathrm{H}]^{+} 474.2142$ 


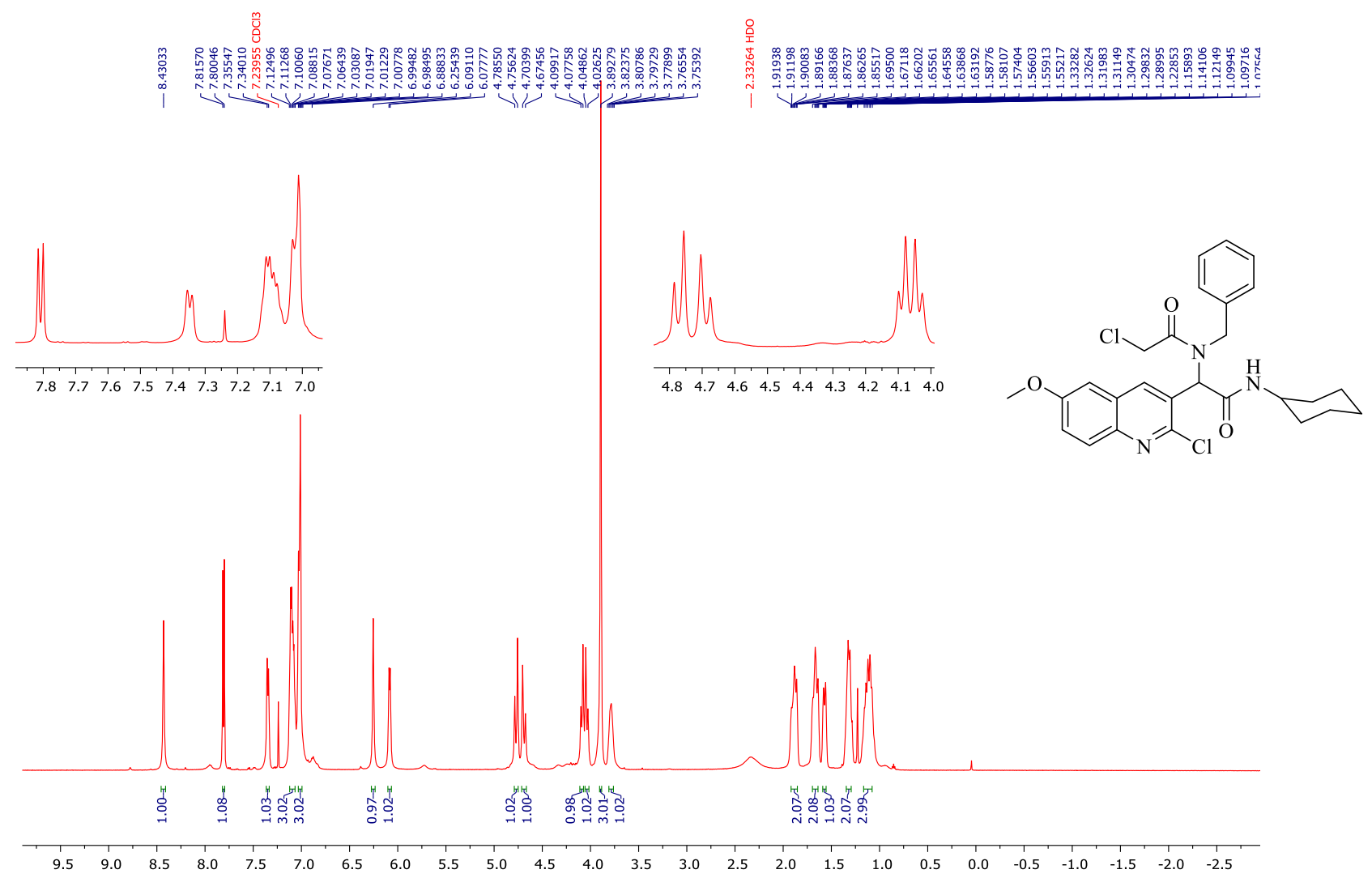

Figure S74: ${ }^{1} \mathrm{H}-\mathrm{NMR}$ of compound $\mathbf{5 u}\left(600 \mathrm{MHz}, \mathrm{CDCl}_{3}\right)$

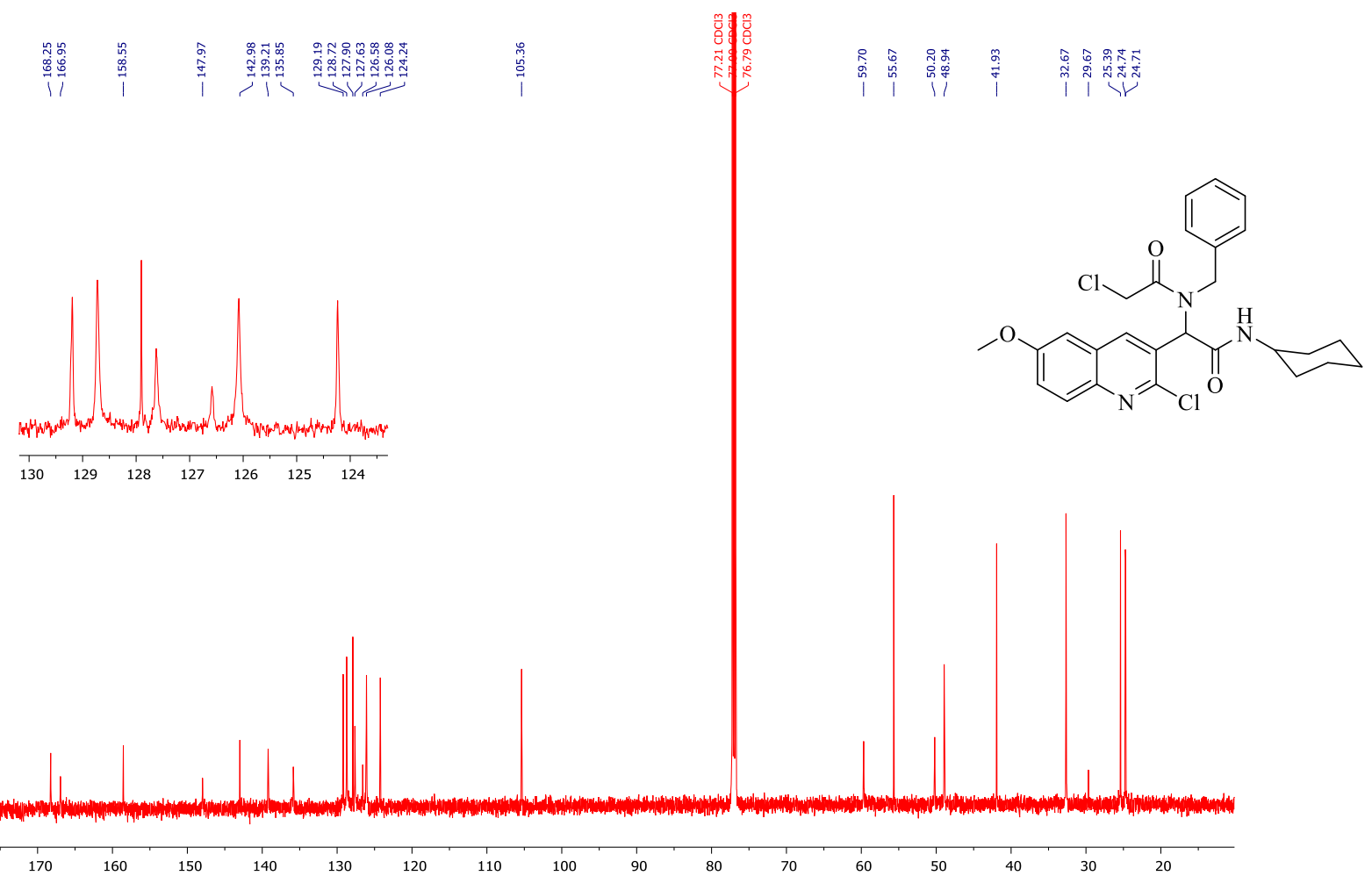

Figure S75: ${ }^{13} \mathrm{C}-\mathrm{NMR}$ of compound $\mathbf{5 u}\left(150 \mathrm{MHz}, \mathrm{CDCl}_{3}\right)$ 


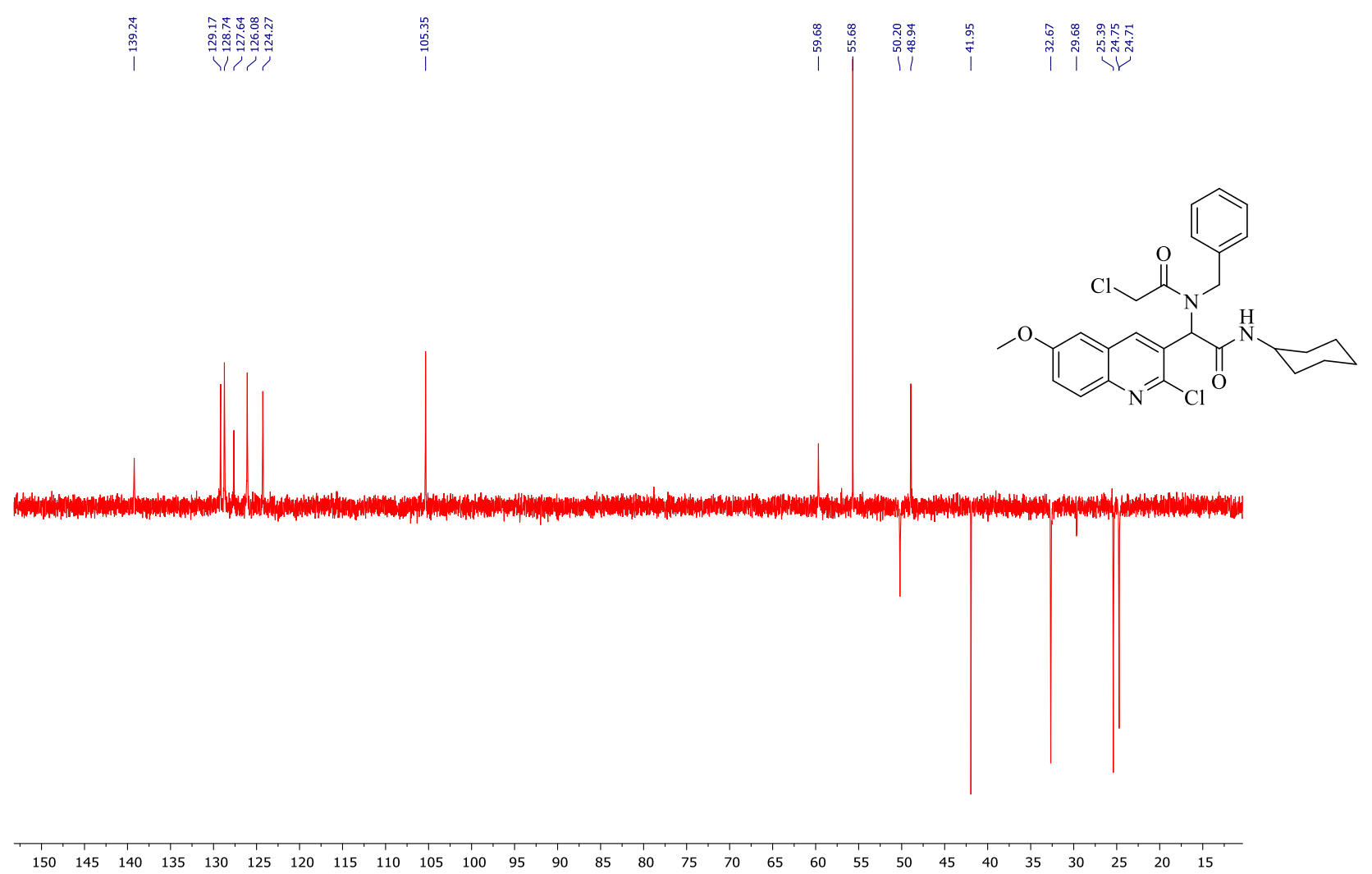

Figure S76: ${ }^{13} \mathrm{C}-\mathrm{NMR}$ (DEPT 135) of compound $5 \mathbf{u}\left(150 \mathrm{MHz}, \mathrm{CDCl}_{3}\right)$

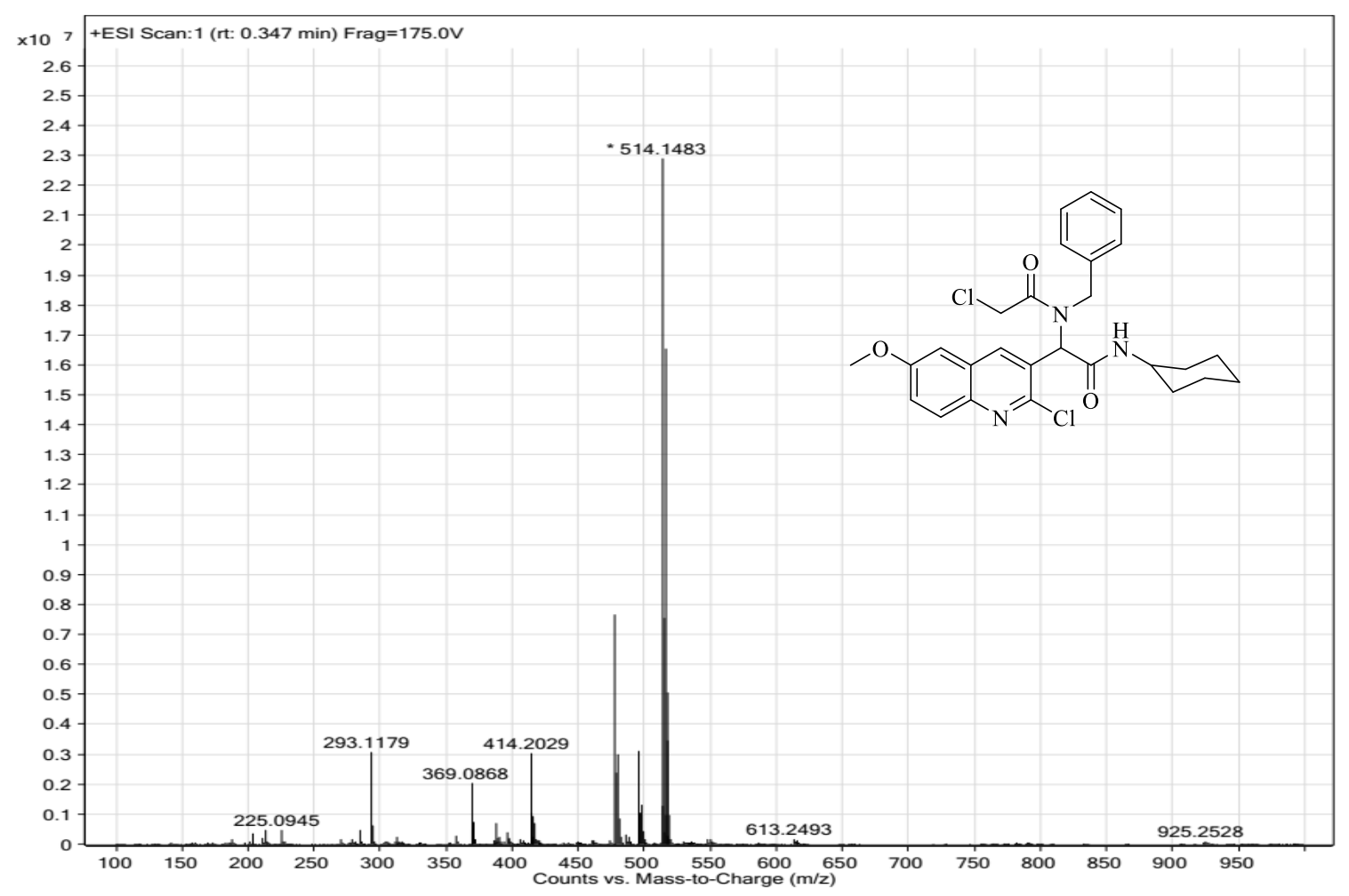

Figure S77: HRMS-ESI of $\mathbf{5 u}$ with formula $\mathrm{C}_{27} \mathrm{H}_{29} \mathrm{Cl}_{2} \mathrm{~N}_{3} \mathrm{O}_{3}$ and $[\mathrm{M}+\mathrm{H}]^{+}$514.1492. 


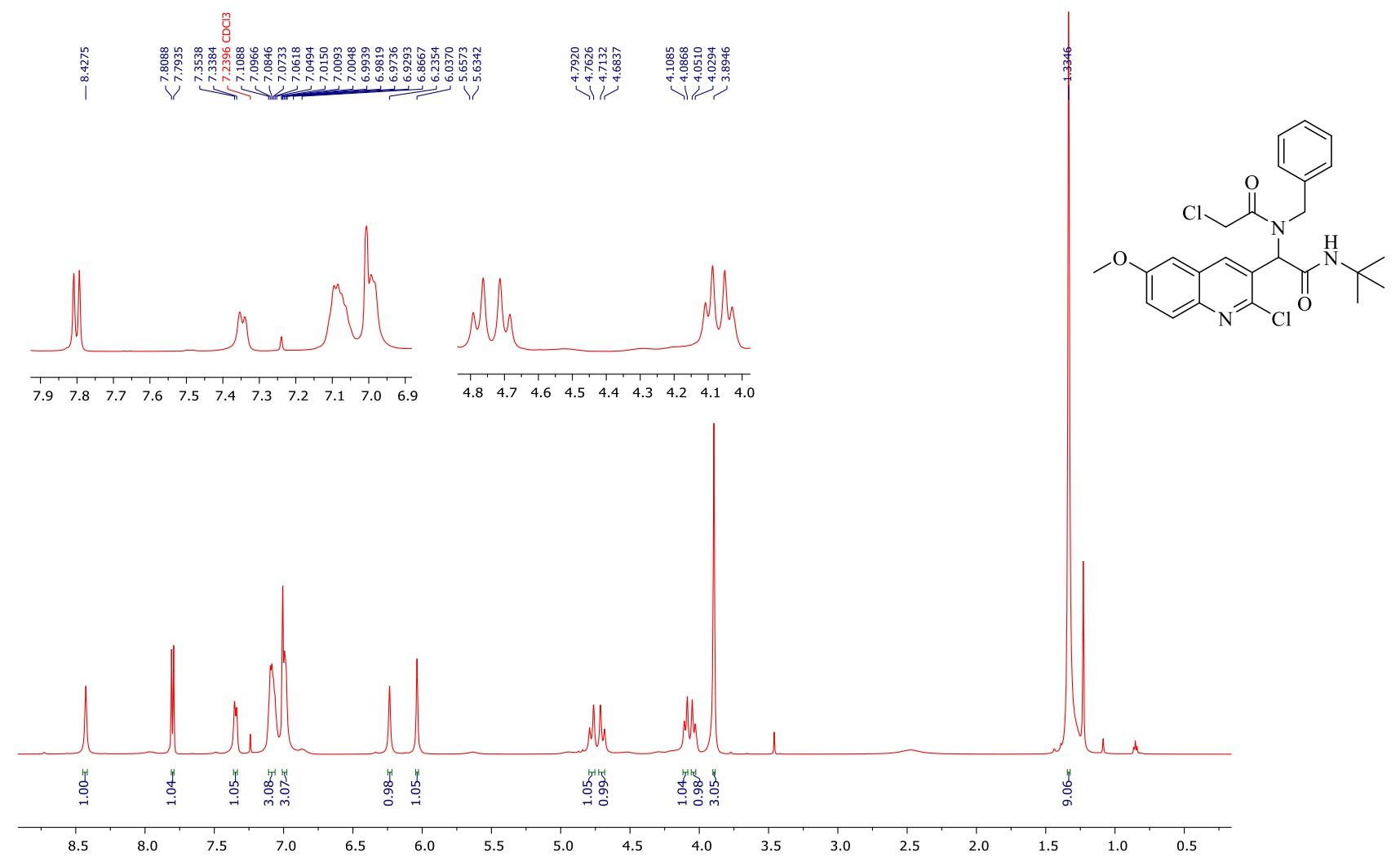

Figure S78: ${ }^{1} \mathrm{H}-\mathrm{NMR}$ of compound $\mathbf{5 v}\left(600 \mathrm{MHz}, \mathrm{CDCl}_{3}\right)$

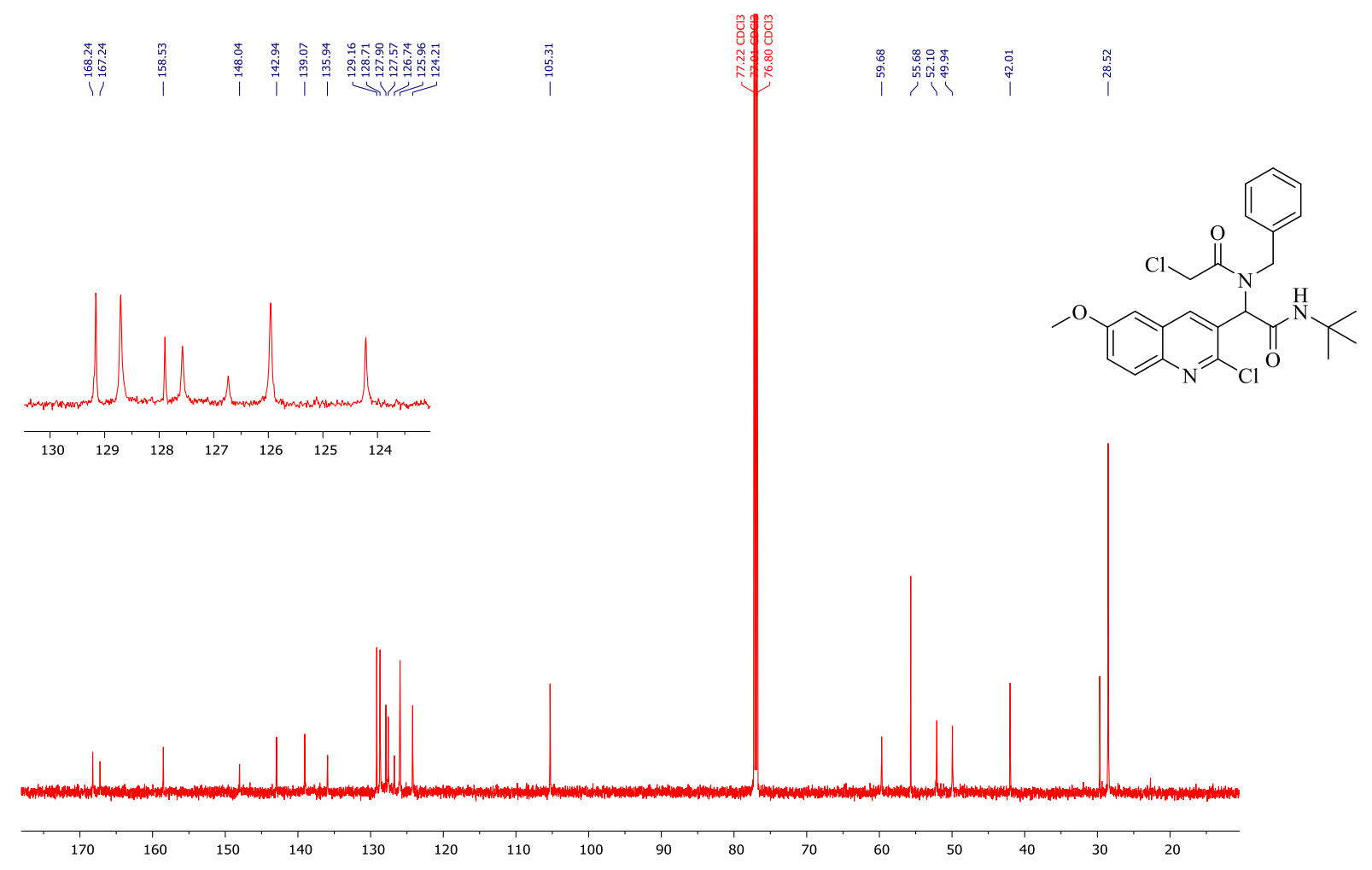

Figure S79: ${ }^{13} \mathrm{C}-\mathrm{NMR}$ of compound $\mathbf{5 v}\left(150 \mathrm{MHz}, \mathrm{CDCl}_{3}\right)$ 


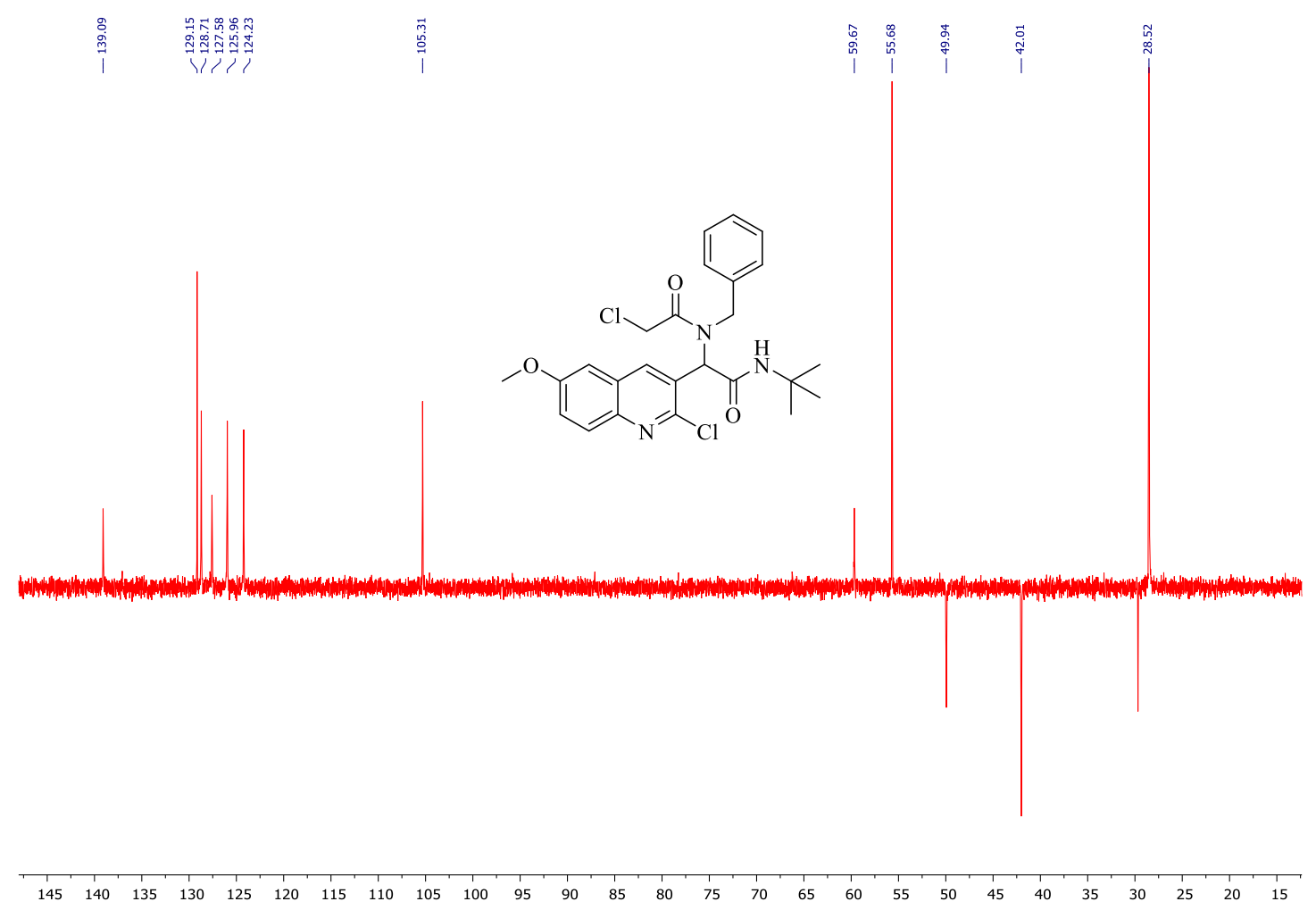

Figure S80: ${ }^{13} \mathrm{C}-\mathrm{NMR}$ (DEPT 135) of compound $\mathbf{5 v}\left(150 \mathrm{MHz}, \mathrm{CDCl}_{3}\right)$

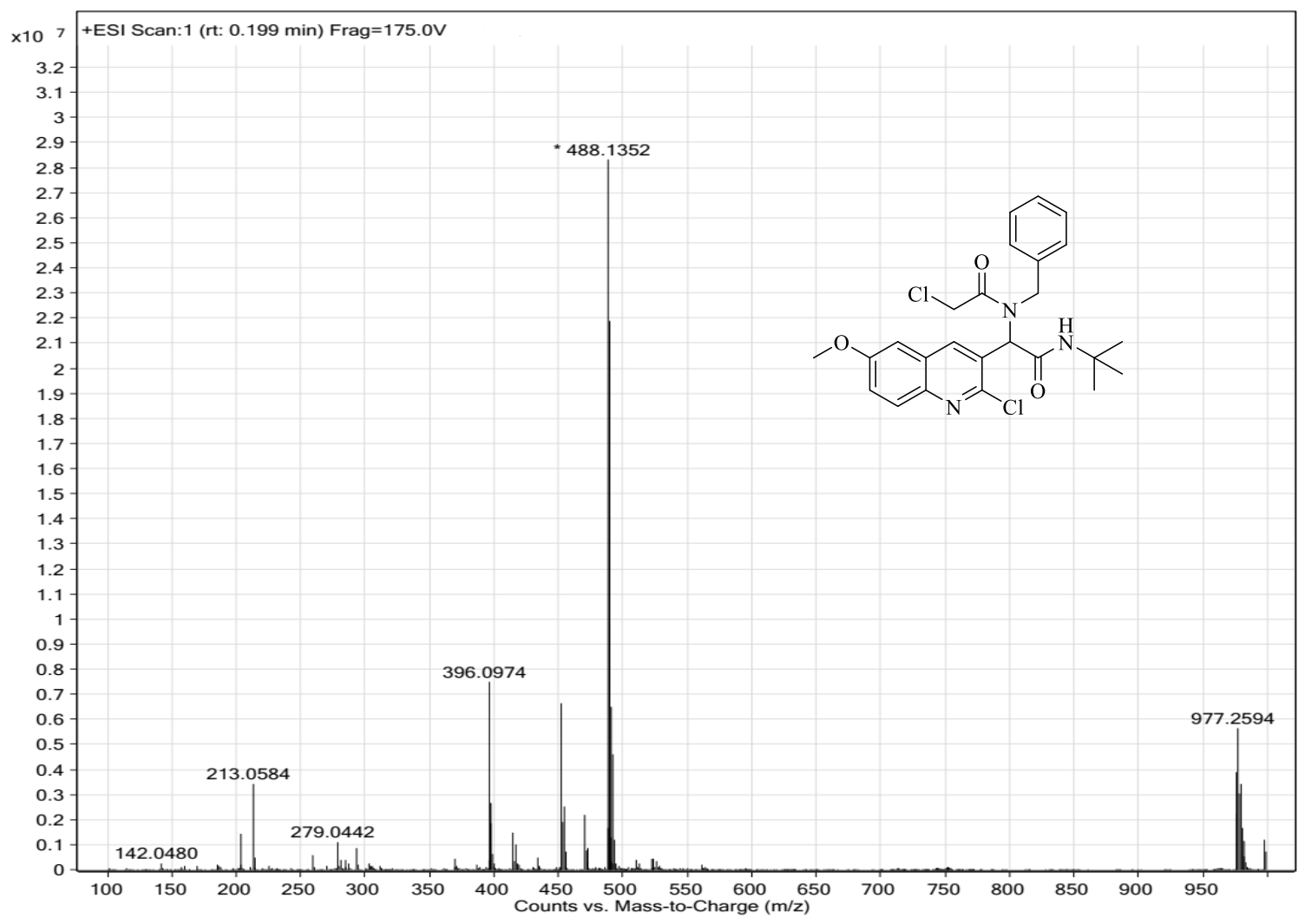

Figure S81: HRMS-ESI of $\mathbf{5 v}$ with formula $\mathrm{C}_{25} \mathrm{H}_{27} \mathrm{Cl}_{2} \mathrm{~N}_{3} \mathrm{O}_{3}$ and $[\mathrm{M}+\mathrm{H}]^{+} 488.1344$. 


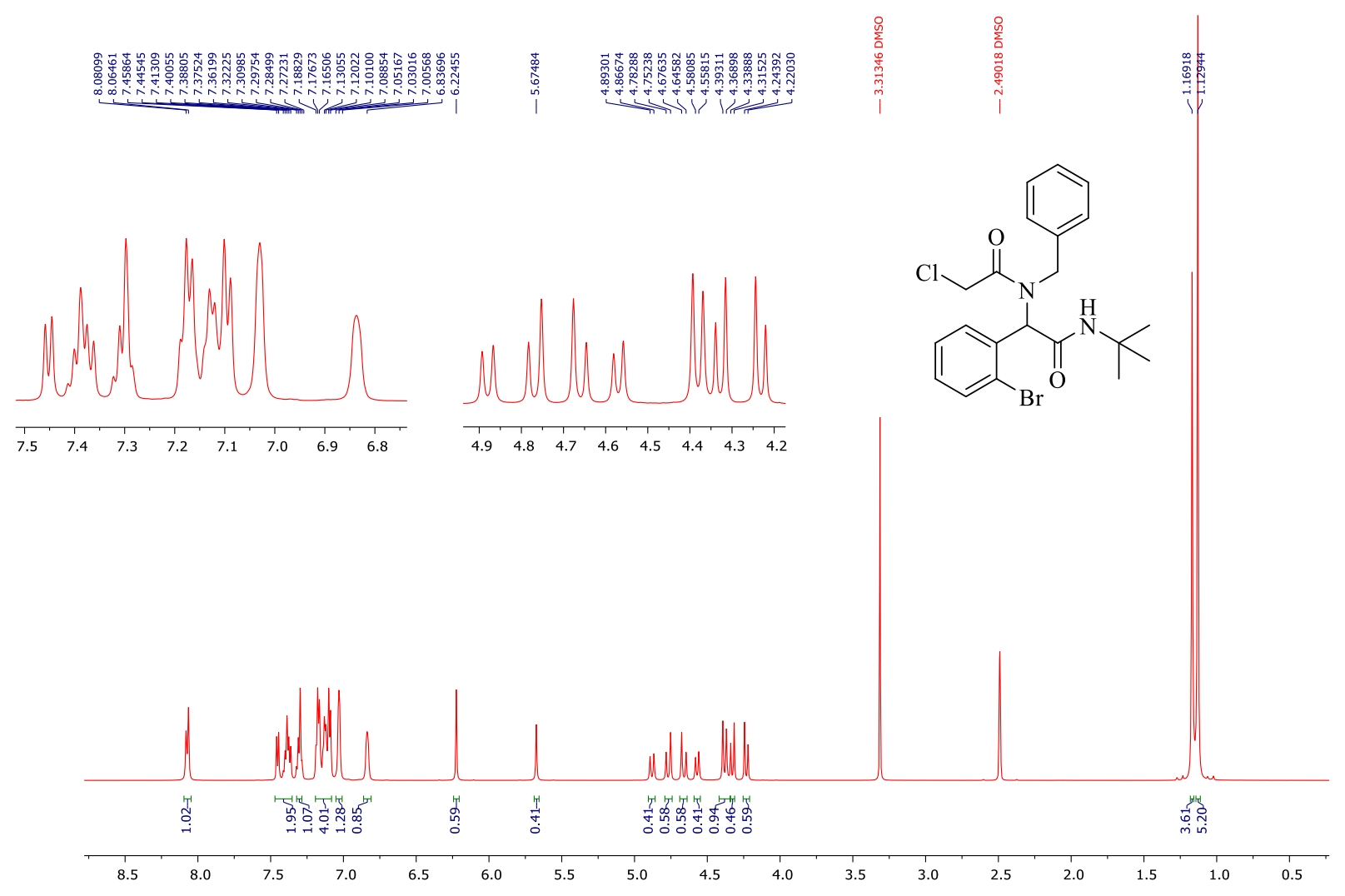

Figure S82: ${ }^{1} \mathrm{H}-\mathrm{NMR}$ of compound $11\left(600 \mathrm{MHz}, \mathrm{DMSO}-d_{6}\right)$
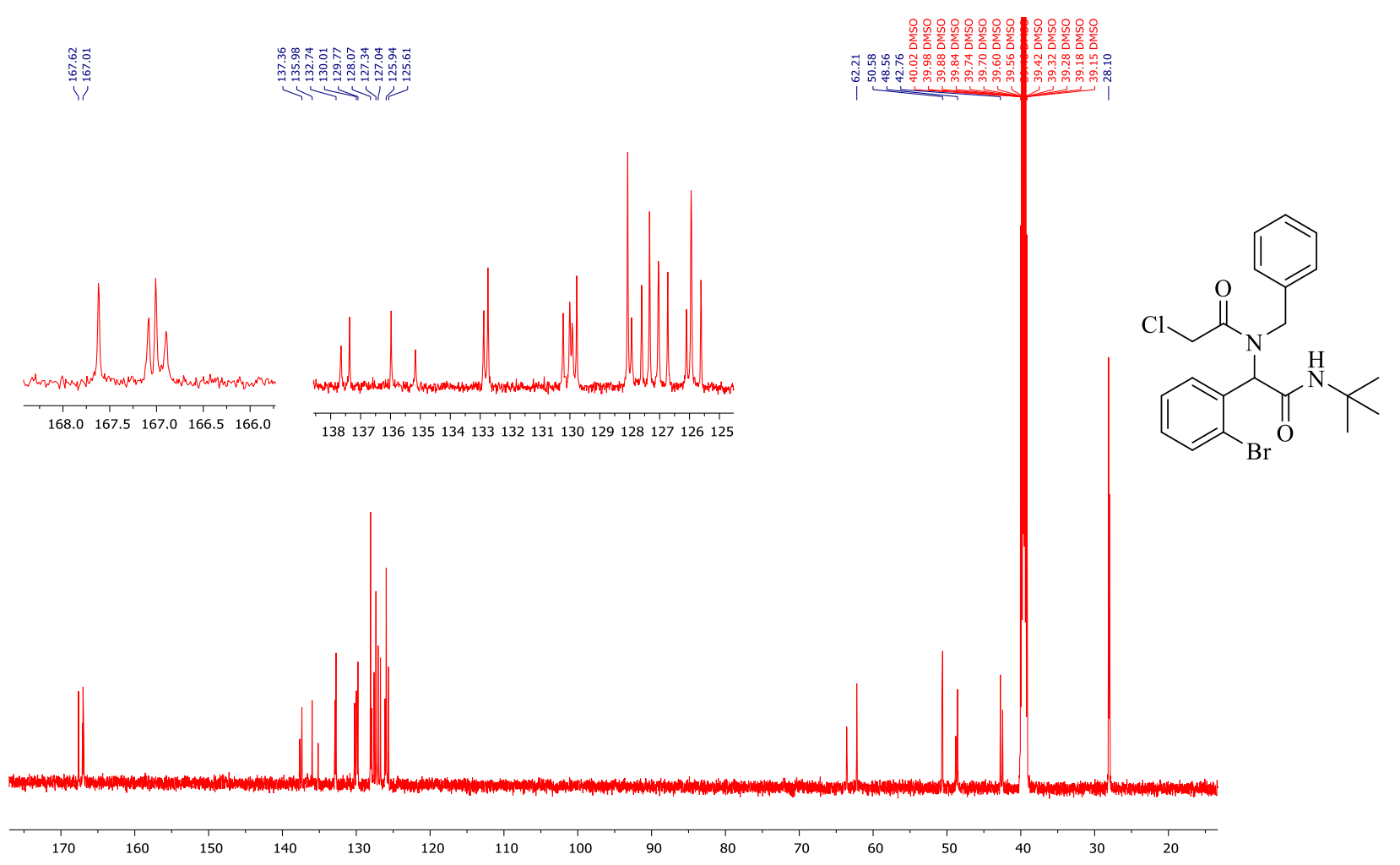

Figure S83: ${ }^{13} \mathrm{C}-\mathrm{NMR}$ of compound 11 (150 MHz, DMSO-d $\left.d_{6}\right)$ 


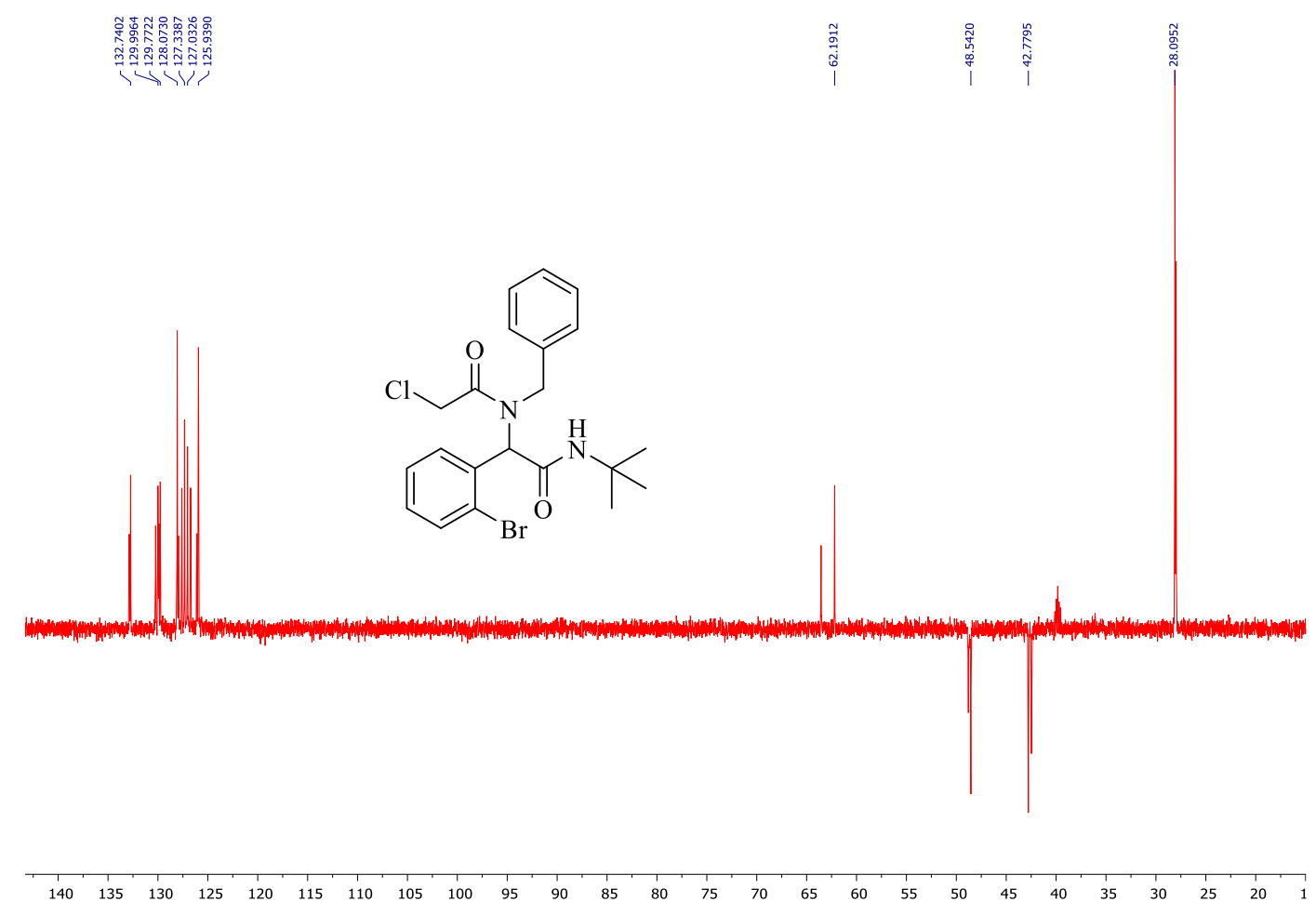

Figure S84: ${ }^{13} \mathrm{C}-\mathrm{NMR}$ (DEPT 135) of compound 11 (150 MHz, DMSO- $\left.d_{6}\right)$

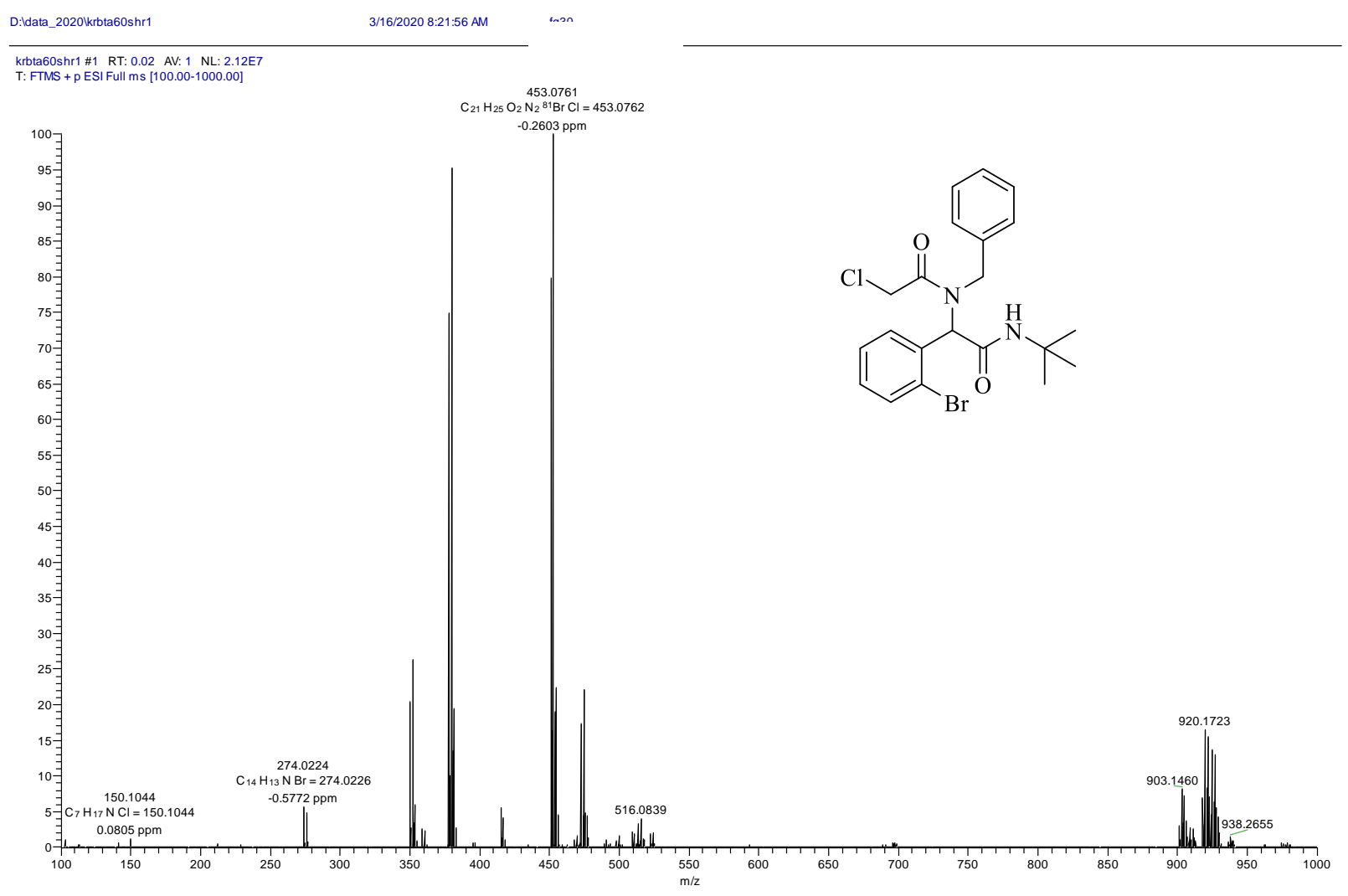

Figure S85: HRMS-ESI of $\mathbf{1 1}$ with formula $\mathrm{C}_{21} \mathrm{H}_{24}{ }^{81} \mathrm{BrClN}_{2} \mathrm{O}_{2}$ and $[\mathrm{M}+\mathrm{H}]^{+} 453.0762$. 


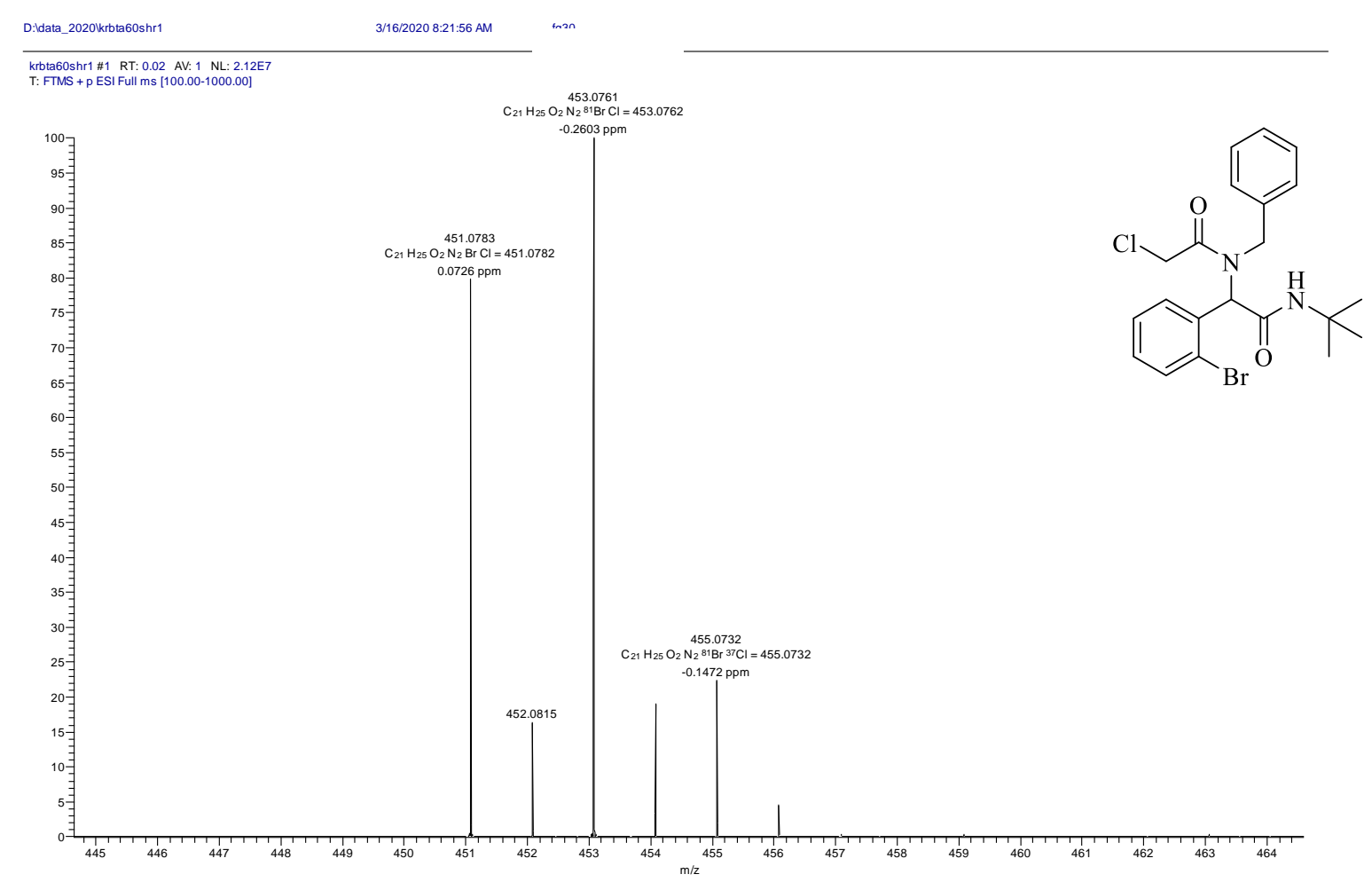

Figure S86: HRMS-ESI of 11 with formula $\mathrm{C}_{21} \mathrm{H}_{24} \mathrm{BrClN}_{2} \mathrm{O}_{2}$ and $[\mathrm{M}+\mathrm{H}]^{+} 451.0782$.

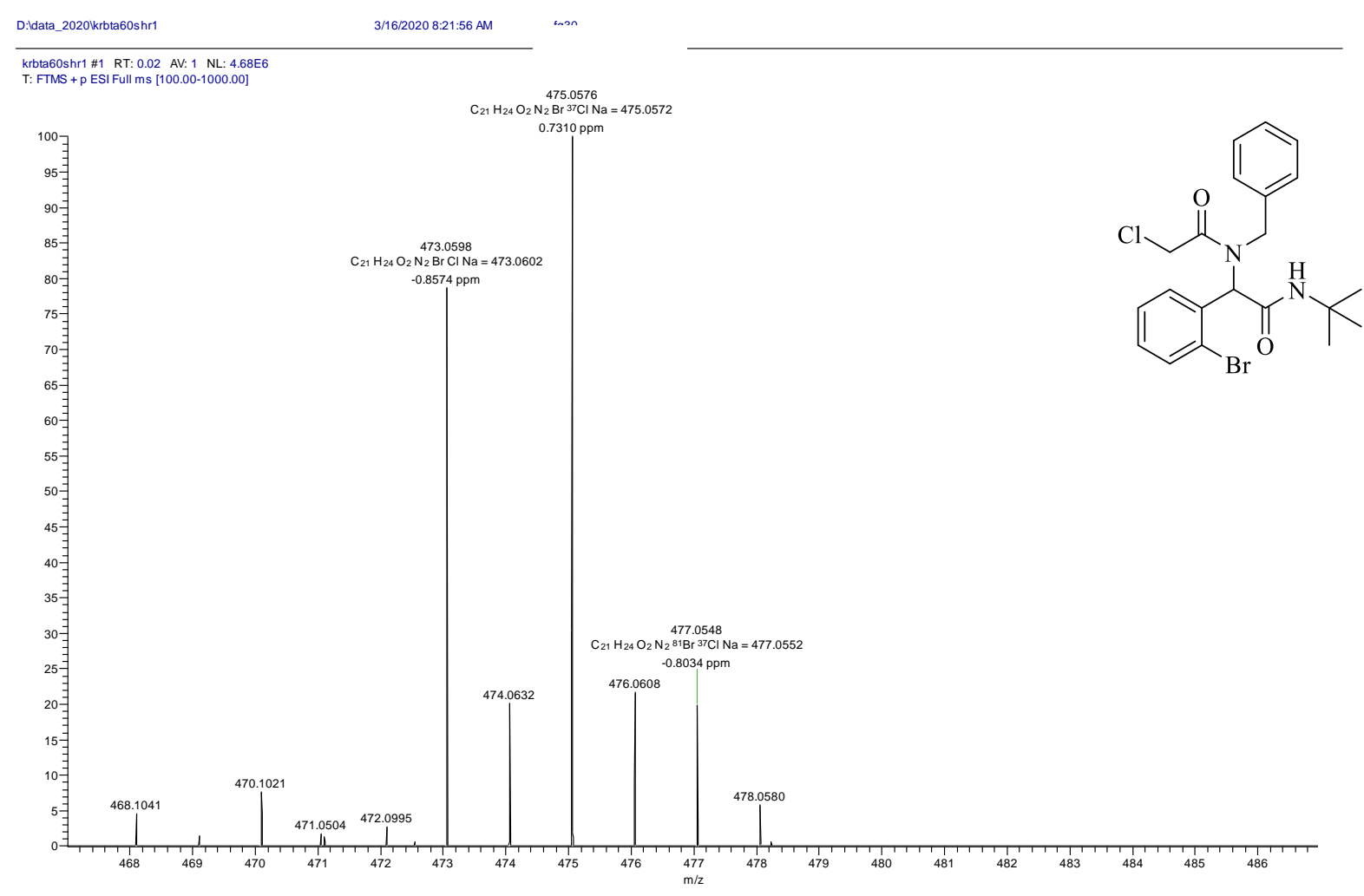

Figure S87: HRMS-ESI of 11 with formula $\mathrm{C}_{21} \mathrm{H}_{24} \mathrm{BrClN}_{2} \mathrm{O}_{2} \mathrm{Na}$ and $[\mathrm{M}+\mathrm{Na}]^{+} 473.0602$. 


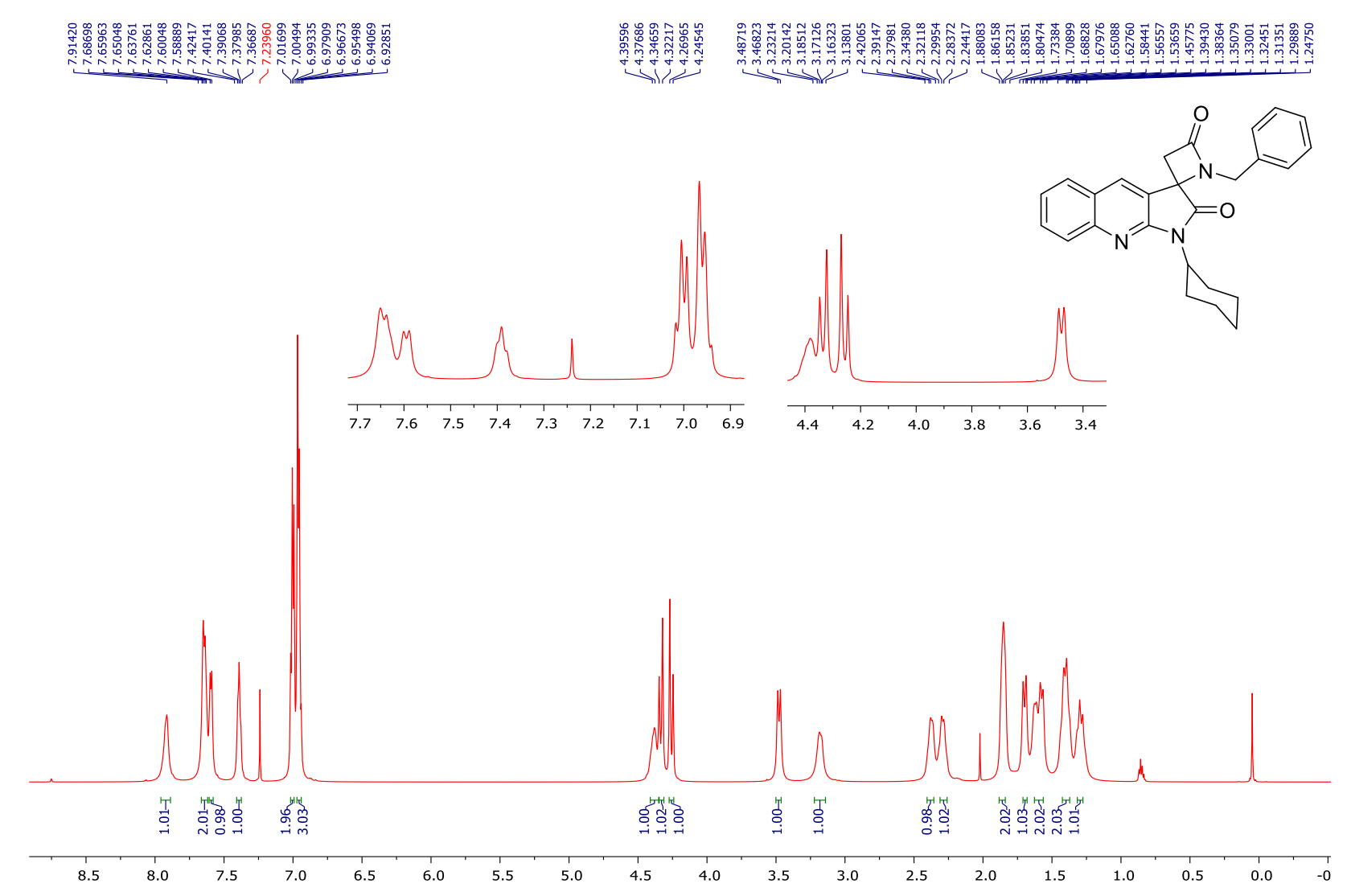

Figure S88: ${ }^{1} \mathrm{H}-\mathrm{NMR}$ of compound $\mathbf{6 a}\left(600 \mathrm{MHz}, \mathrm{CDCl}_{3}\right)$

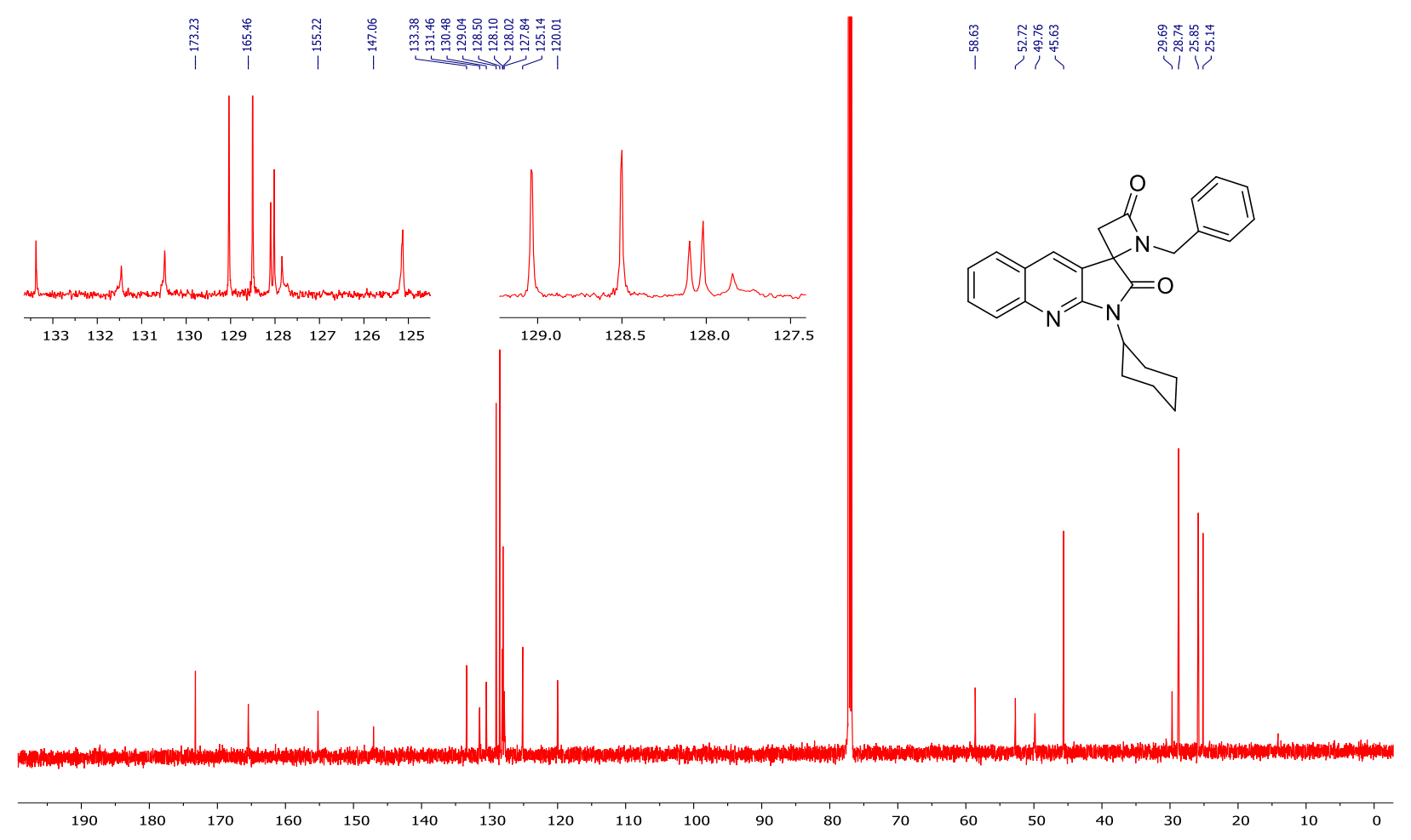

Figure S89: ${ }^{13} \mathrm{C}-\mathrm{NMR}$ of compound $\mathbf{6 a}\left(150 \mathrm{MHz}, \mathrm{CDCl}_{3}\right)$ 


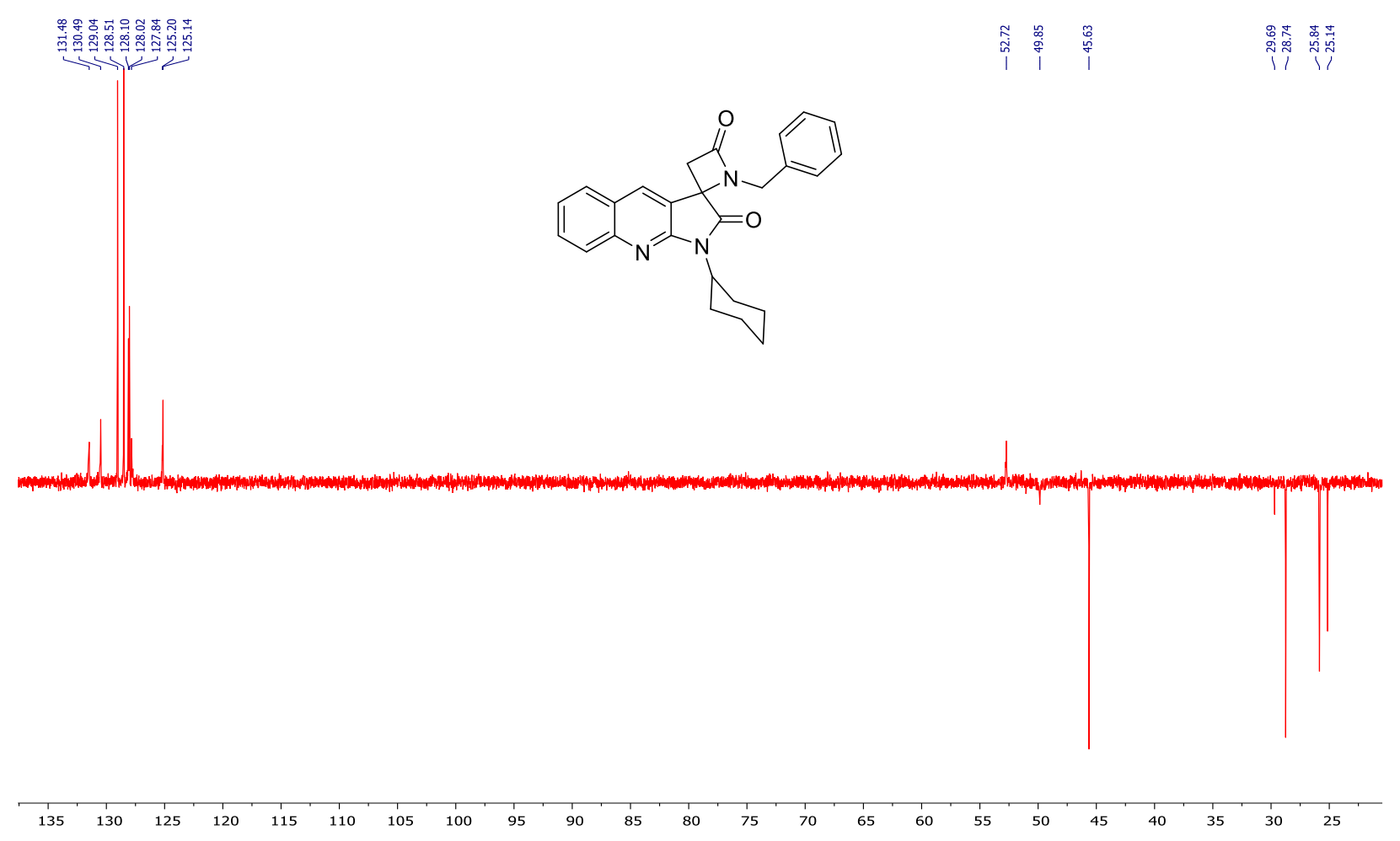

Figure S90: ${ }^{13} \mathrm{C}-\mathrm{NMR}$ (DEPT 135) of compound 6a (150 MHz, $\left.\mathrm{CDCl}_{3}\right)$

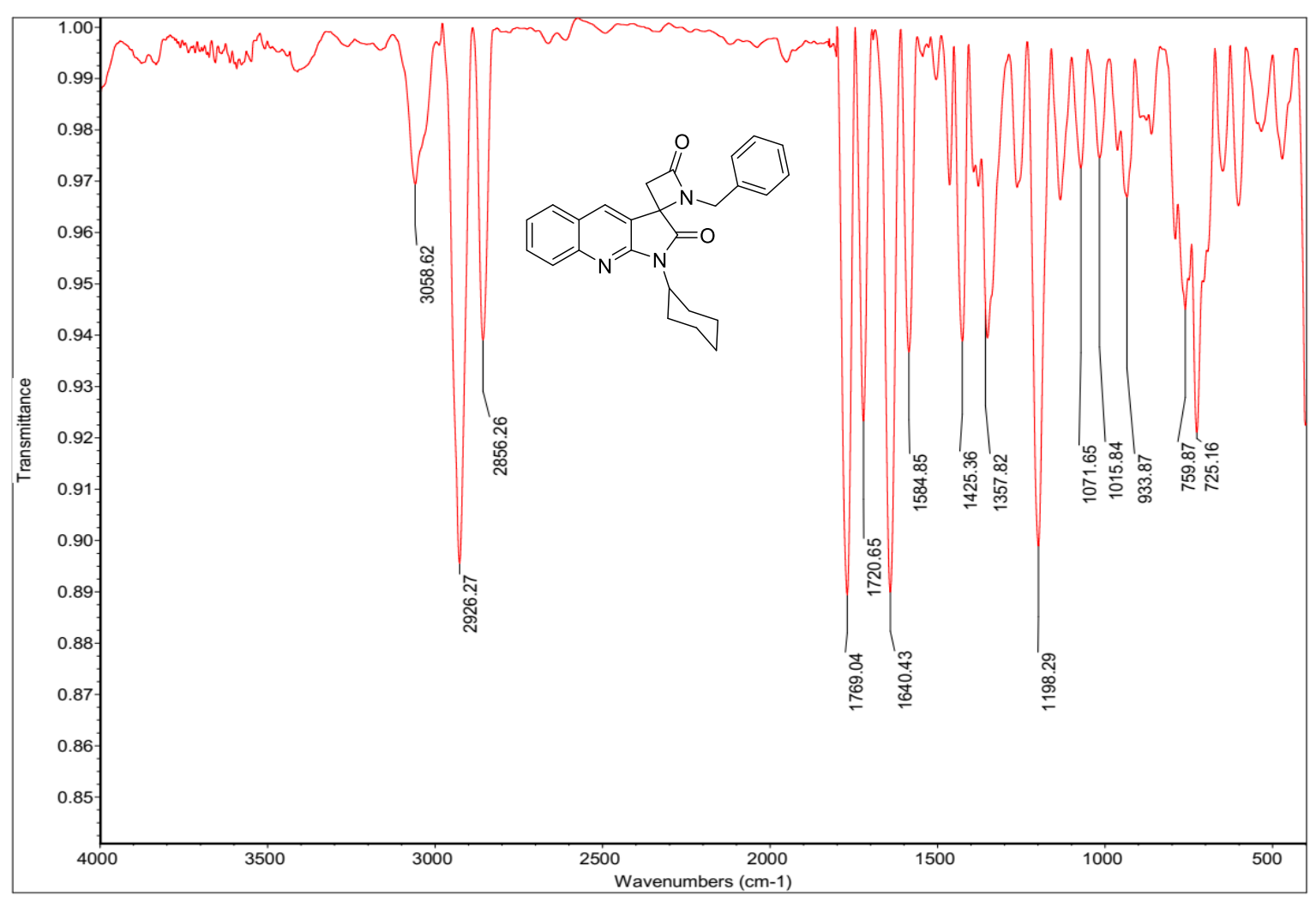

Figure S91: IR of compound $6 \mathrm{a}\left(\mathrm{KBr}, \mathrm{cm}^{-1}\right)$ 


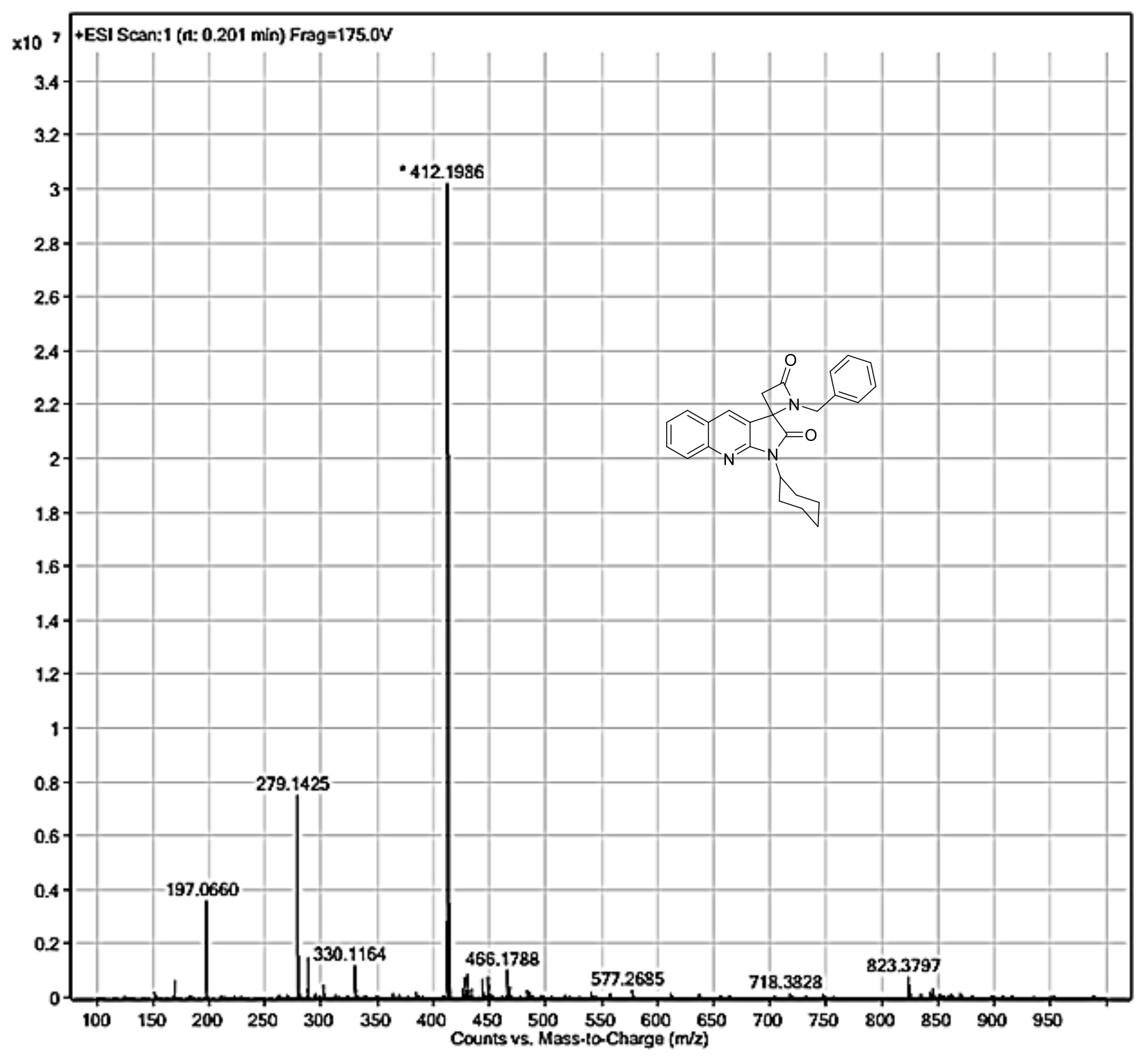

Figure S92: HRMS-ESI of $6 \mathbf{a}$ with formula $\mathrm{C}_{26} \mathrm{H}_{25} \mathrm{~N}_{3} \mathrm{O}_{2}$ and $[\mathrm{M}+\mathrm{H}]^{+} 412.1977$ 

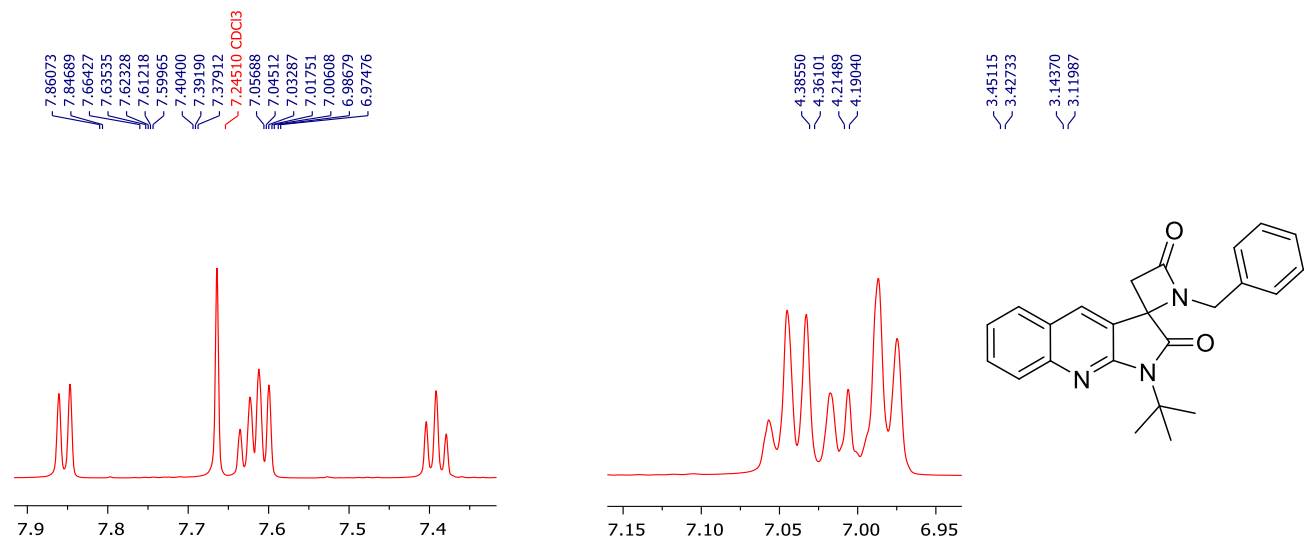

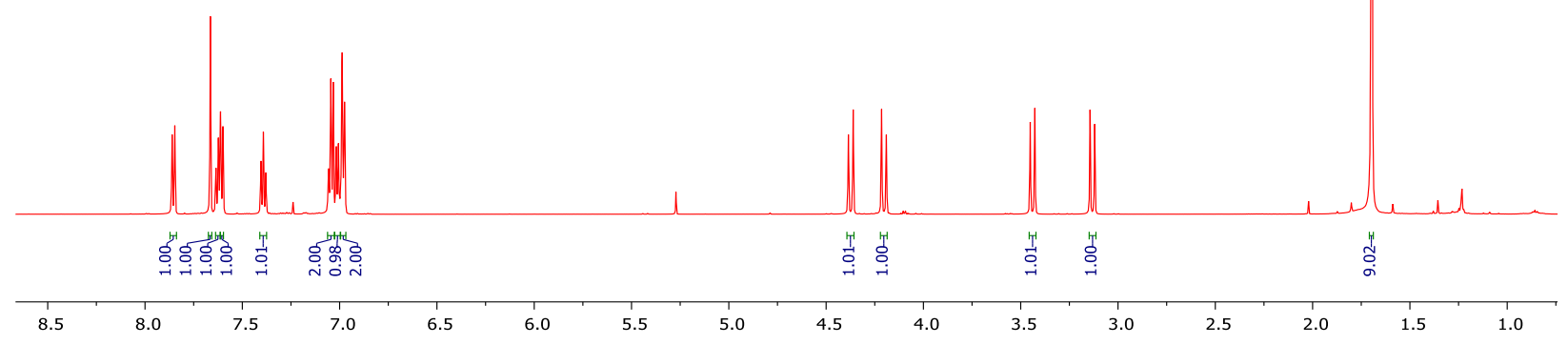

Figure S93: ${ }^{1} \mathrm{H}-\mathrm{NMR}$ of compound $\mathbf{6 b}\left(600 \mathrm{MHz}, \mathrm{CDCl}_{3}\right)$

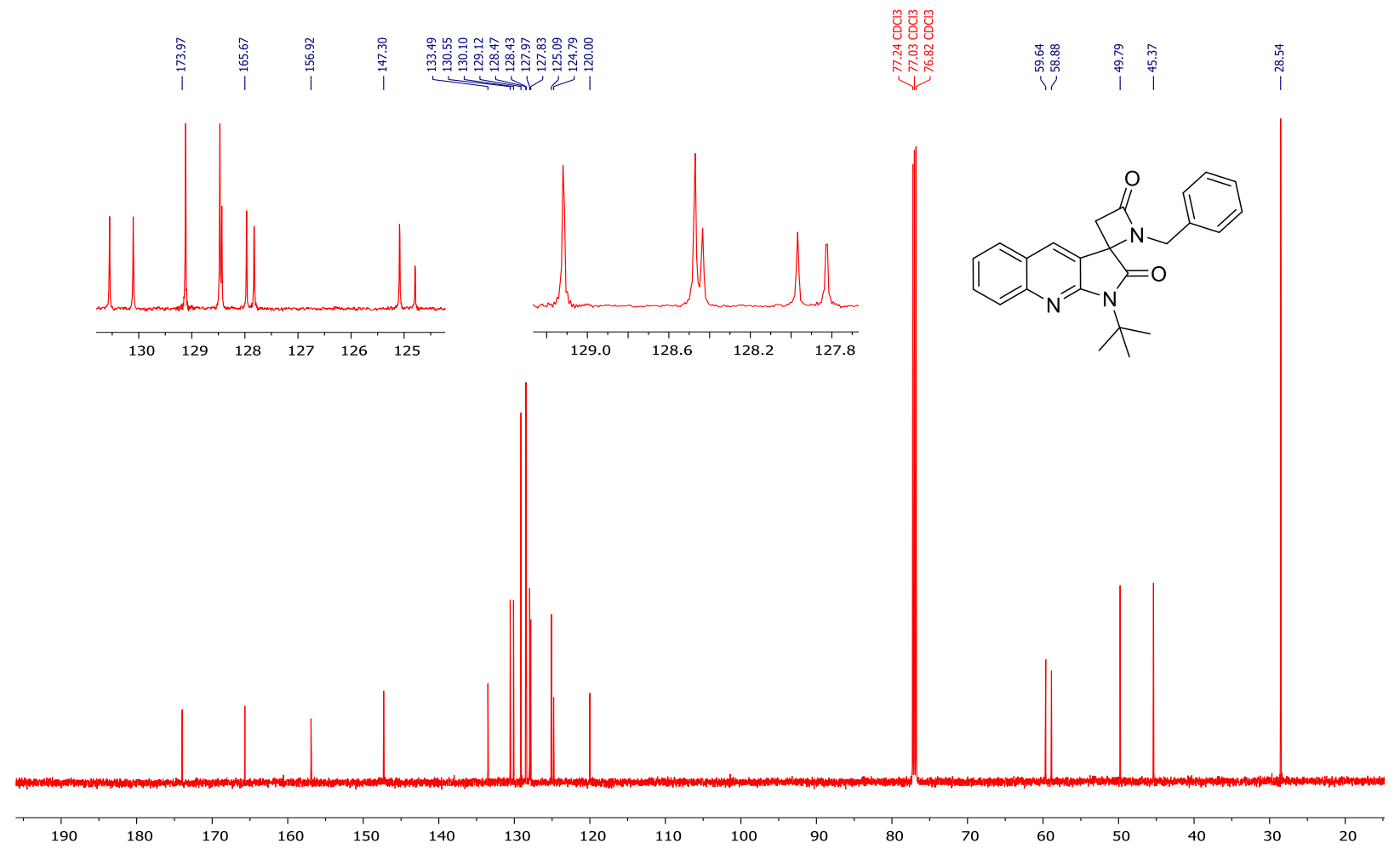

Figure S94: ${ }^{13} \mathrm{C}-\mathrm{NMR}$ of compound $\mathbf{6 b}\left(150 \mathrm{MHz}, \mathrm{CDCl}_{3}\right)$ 


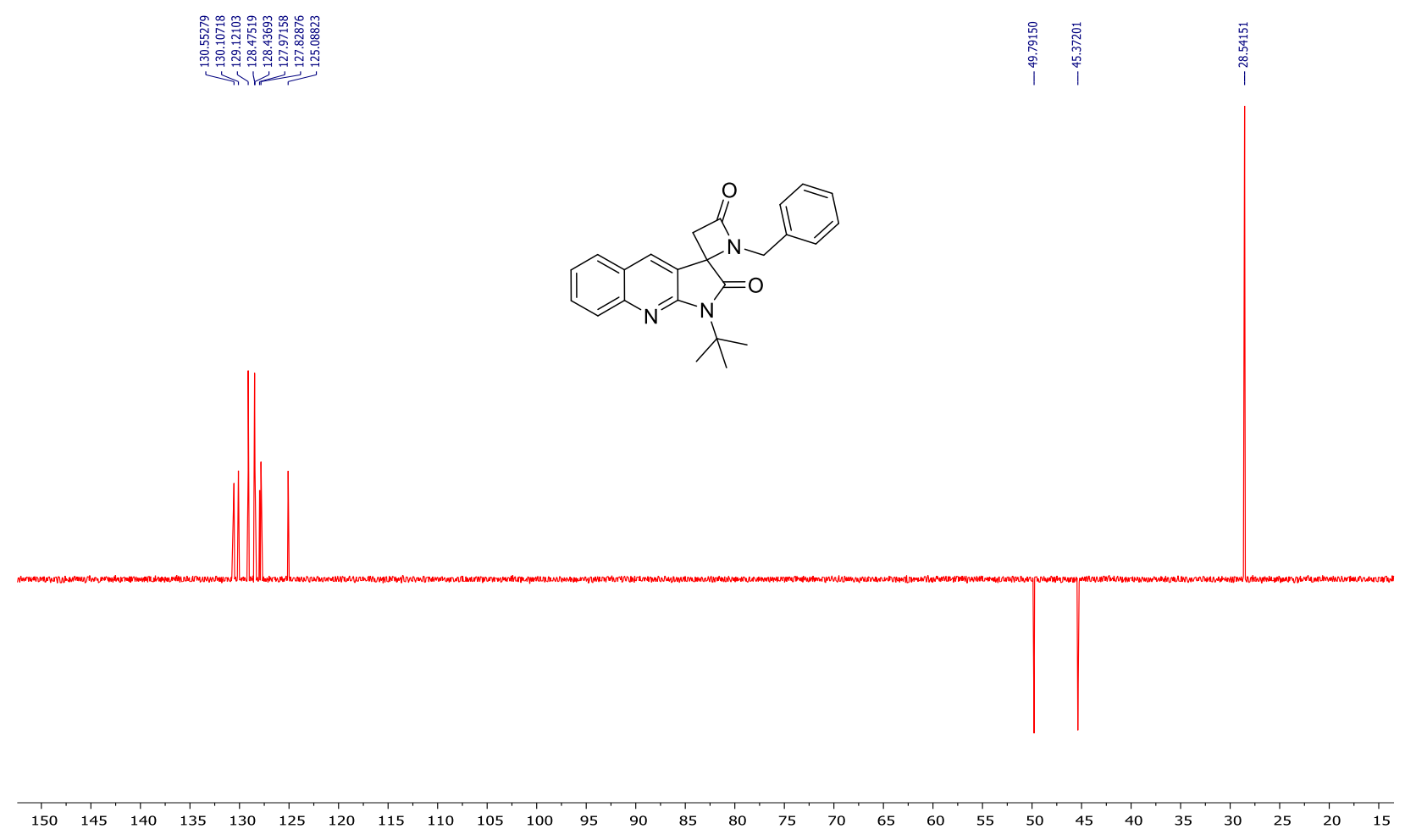

Figure S95: ${ }^{13} \mathrm{C}-\mathrm{NMR}$ (DEPT 135) of compound $\mathbf{6 b}\left(150 \mathrm{MHz}, \mathrm{CDCl}_{3}\right)$

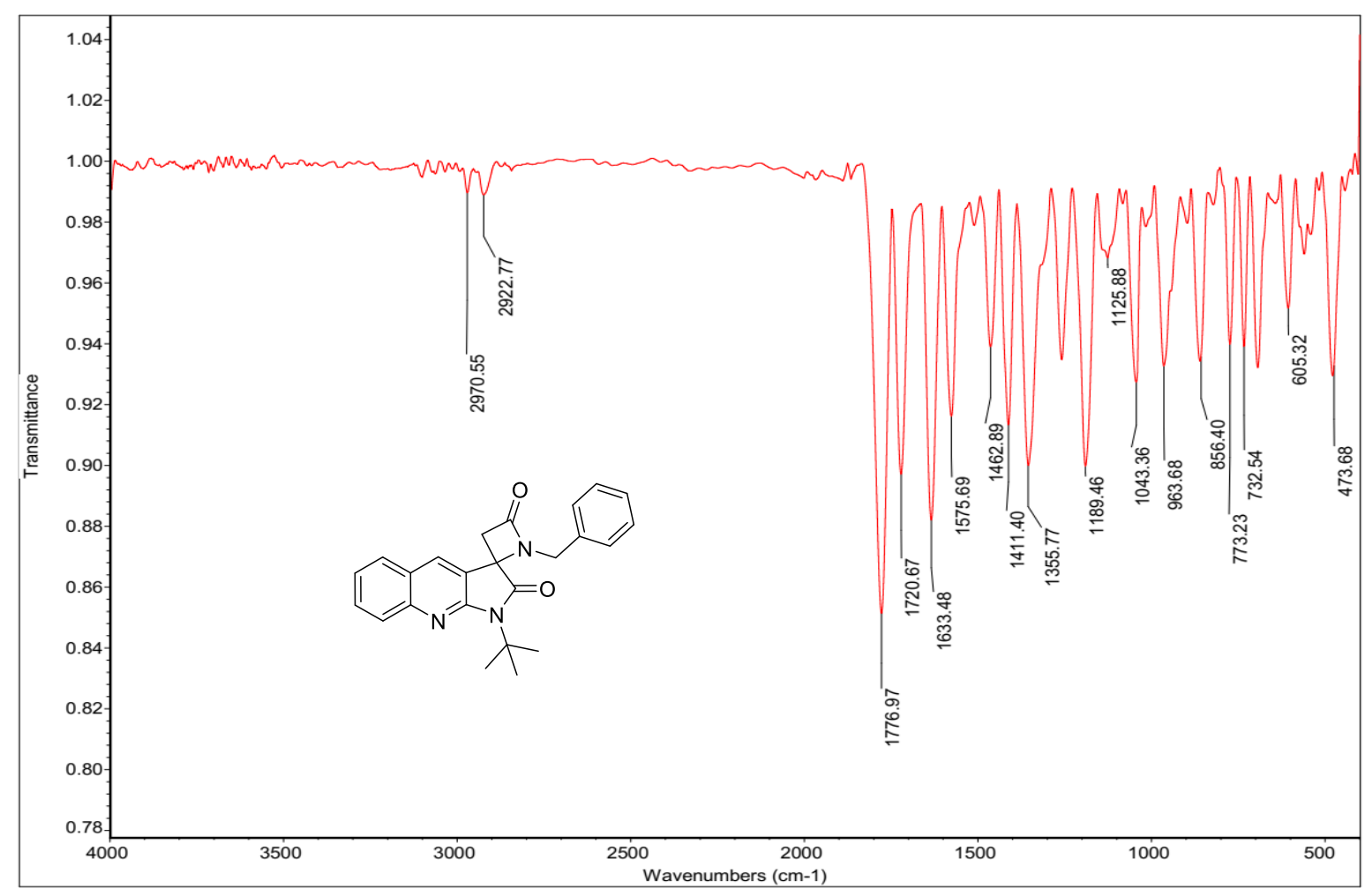

Figure S96: IR of compound $6 \mathrm{~b}\left(\mathrm{KBr}, \mathrm{cm}^{-1}\right)$ 


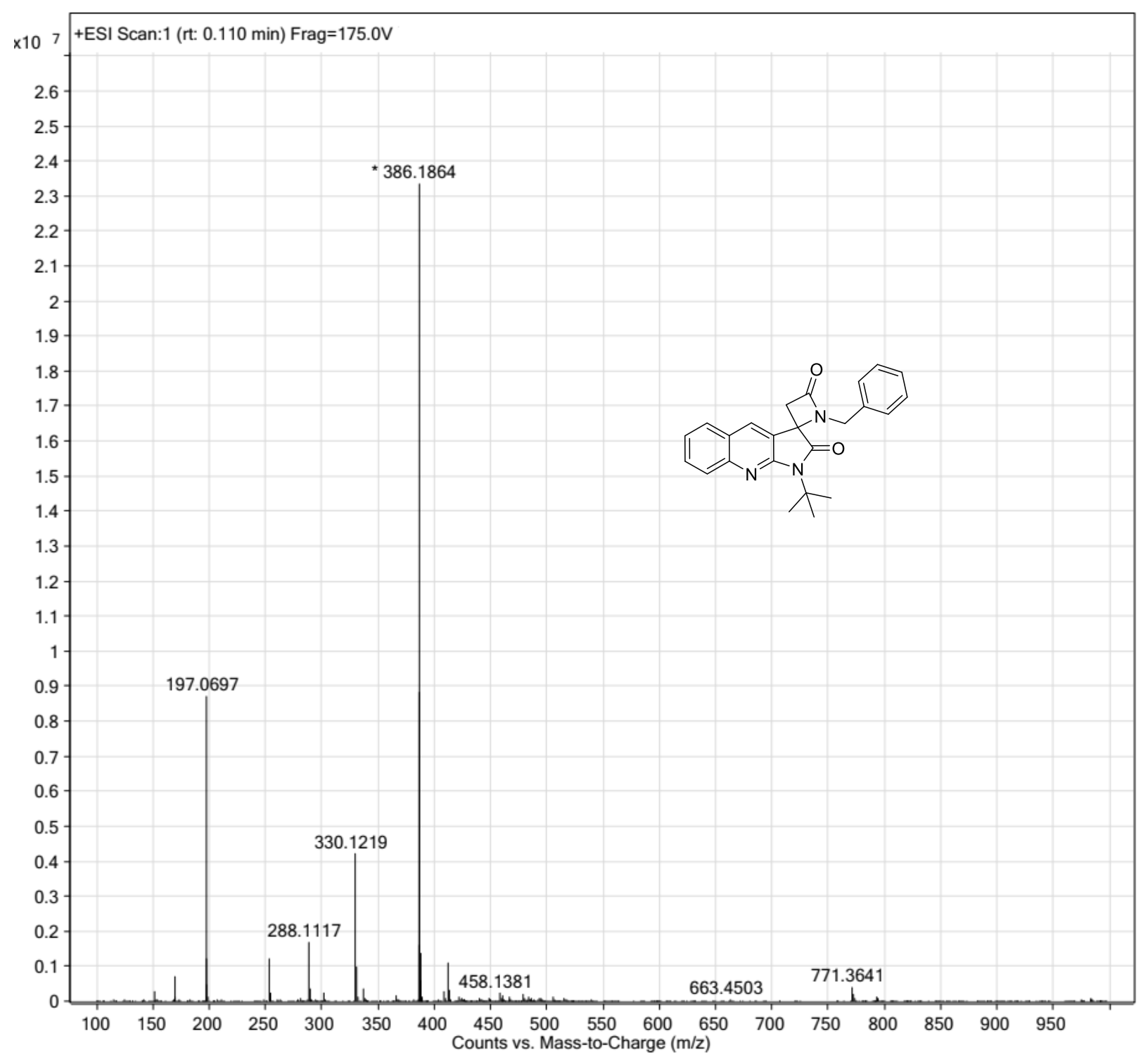

Figure S97: HRMS-ESI of $\mathbf{6 b}$ with formula $\mathrm{C}_{24} \mathrm{H}_{23} \mathrm{~N}_{3} \mathrm{O}_{2}$ and $[\mathrm{M}+\mathrm{H}]^{+} 386.1869$ 

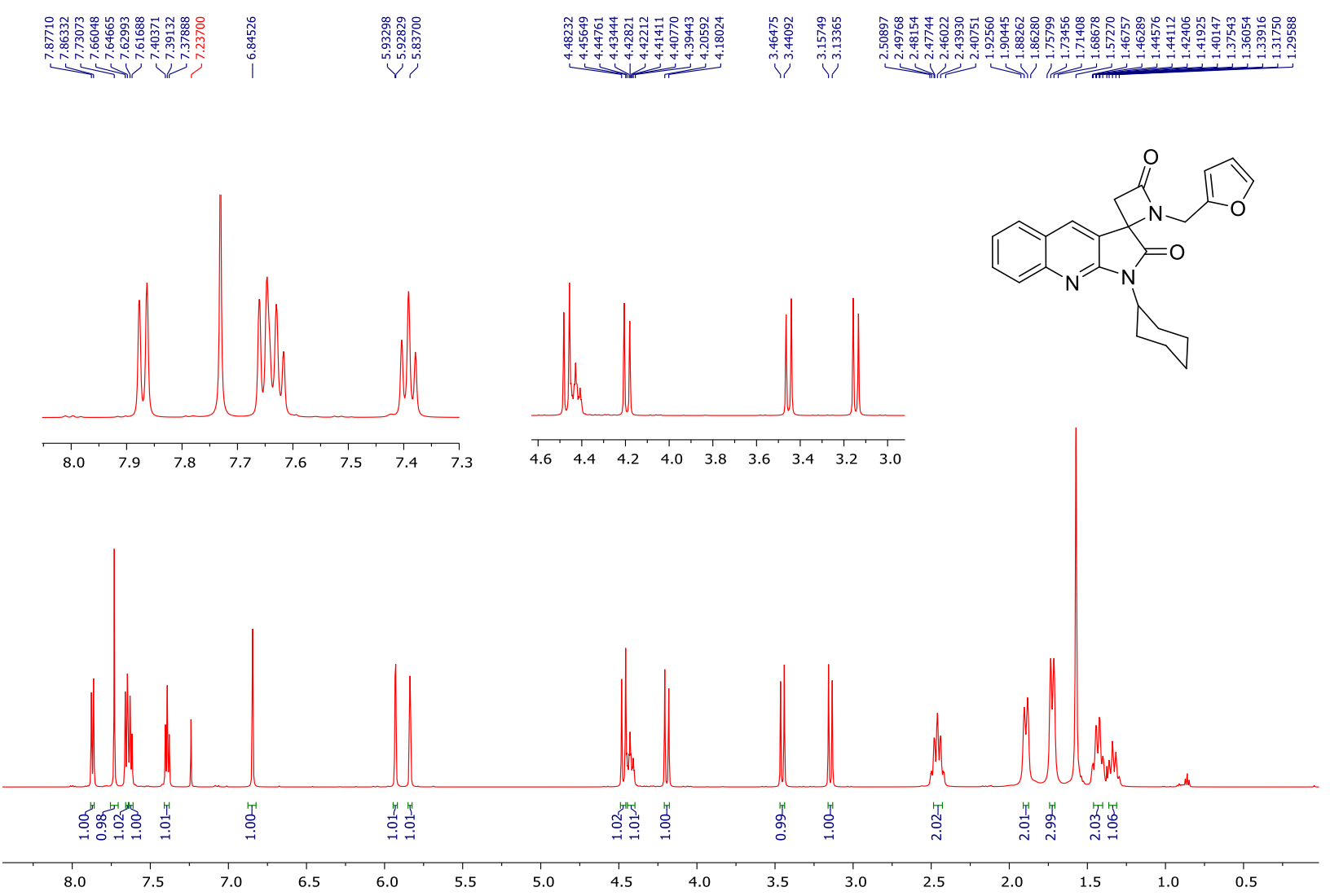

Figure S98: ${ }^{1} \mathrm{H}-\mathrm{NMR}$ of compound $\mathbf{6 c}\left(600 \mathrm{MHz}, \mathrm{CDCl}_{3}\right)$
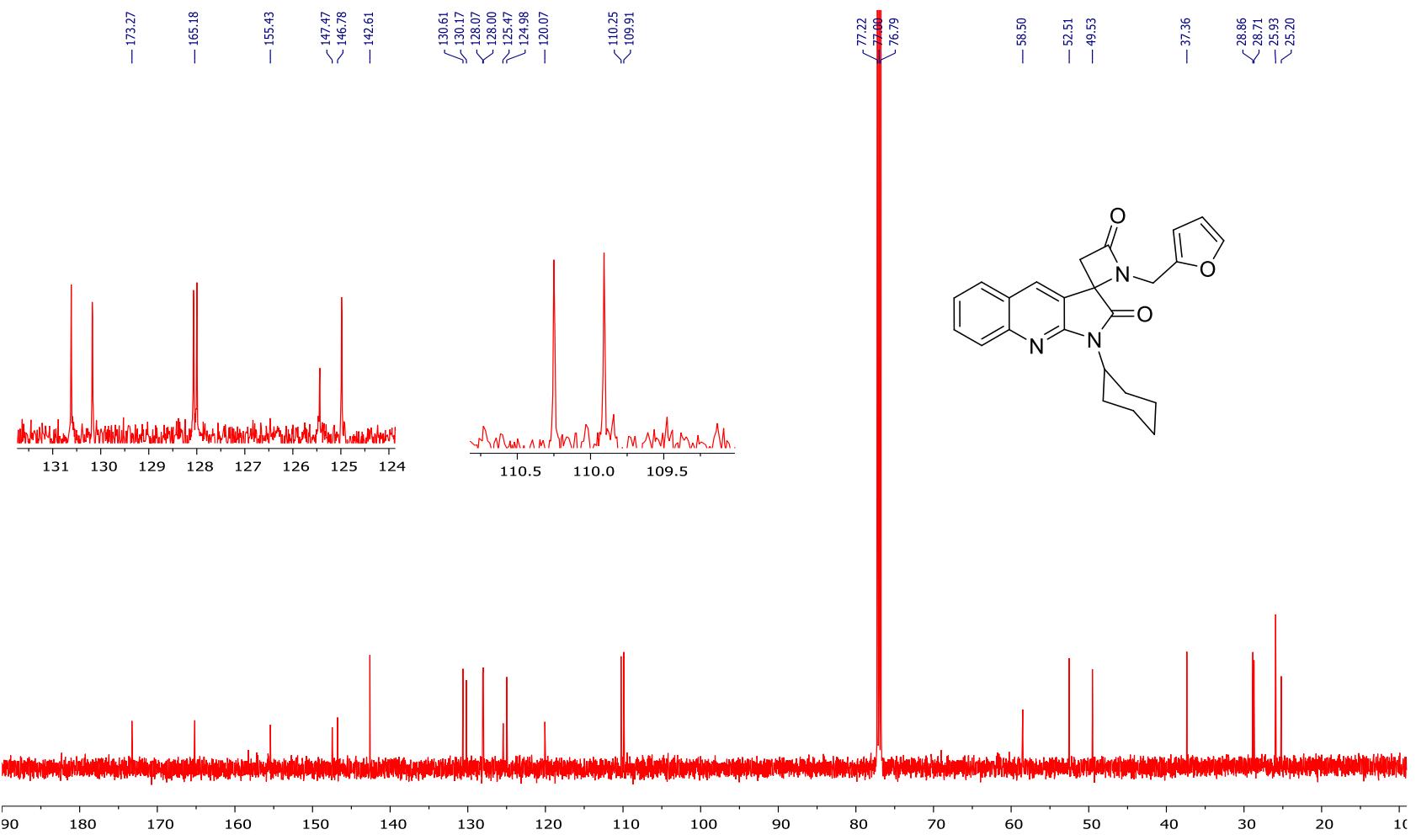

Figure S99: ${ }^{13} \mathrm{C}-\mathrm{NMR}$ of compound $\mathbf{6 c}\left(150 \mathrm{MHz}, \mathrm{CDCl}_{3}\right)$ 

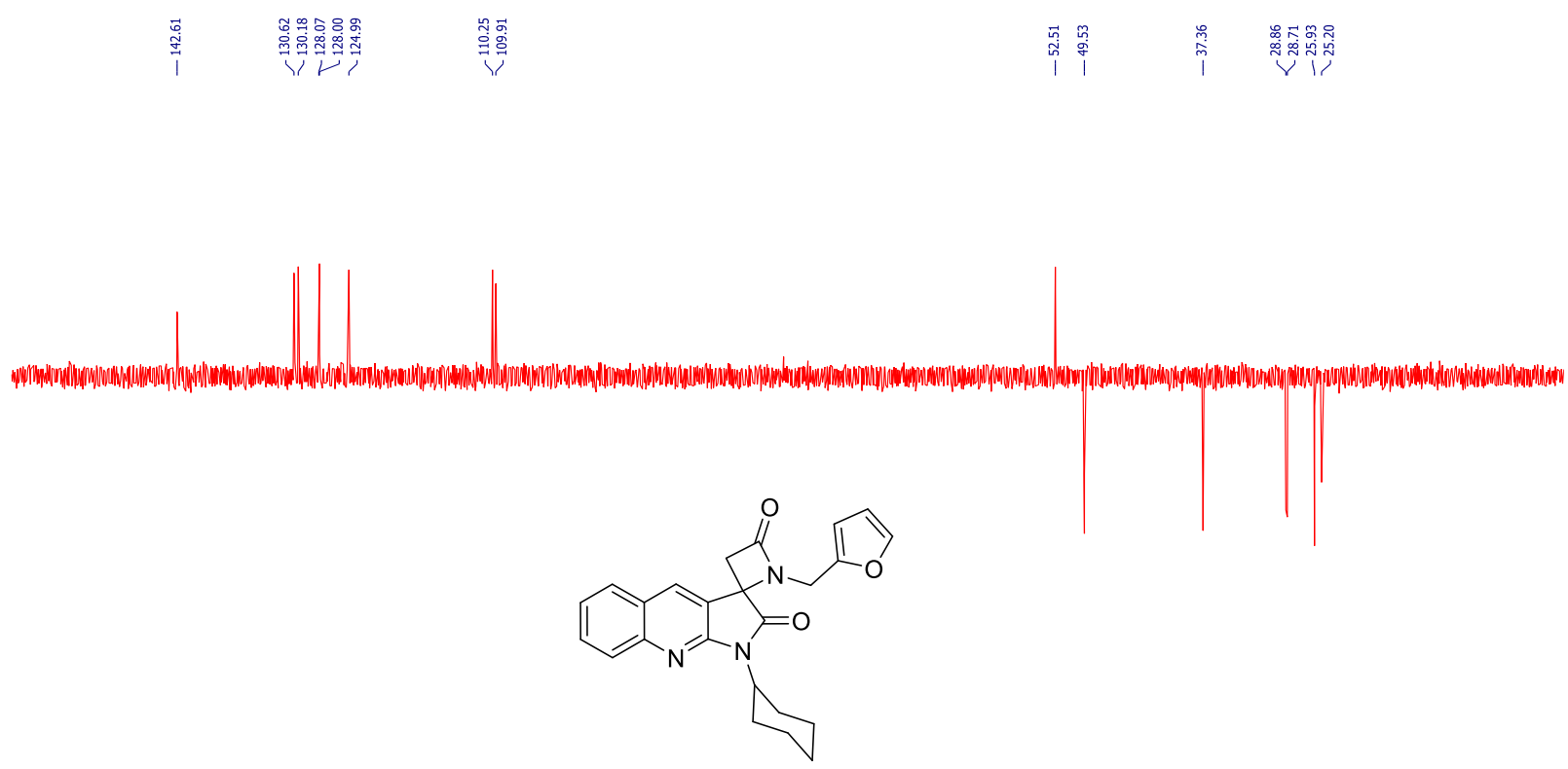

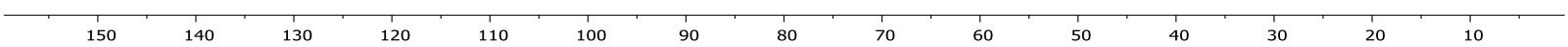

Figure S100: ${ }^{13} \mathrm{C}-\mathrm{NMR}$ (DEPT 135) of compound $\mathbf{6 c}\left(150 \mathrm{MHz}, \mathrm{CDCl}_{3}\right)$

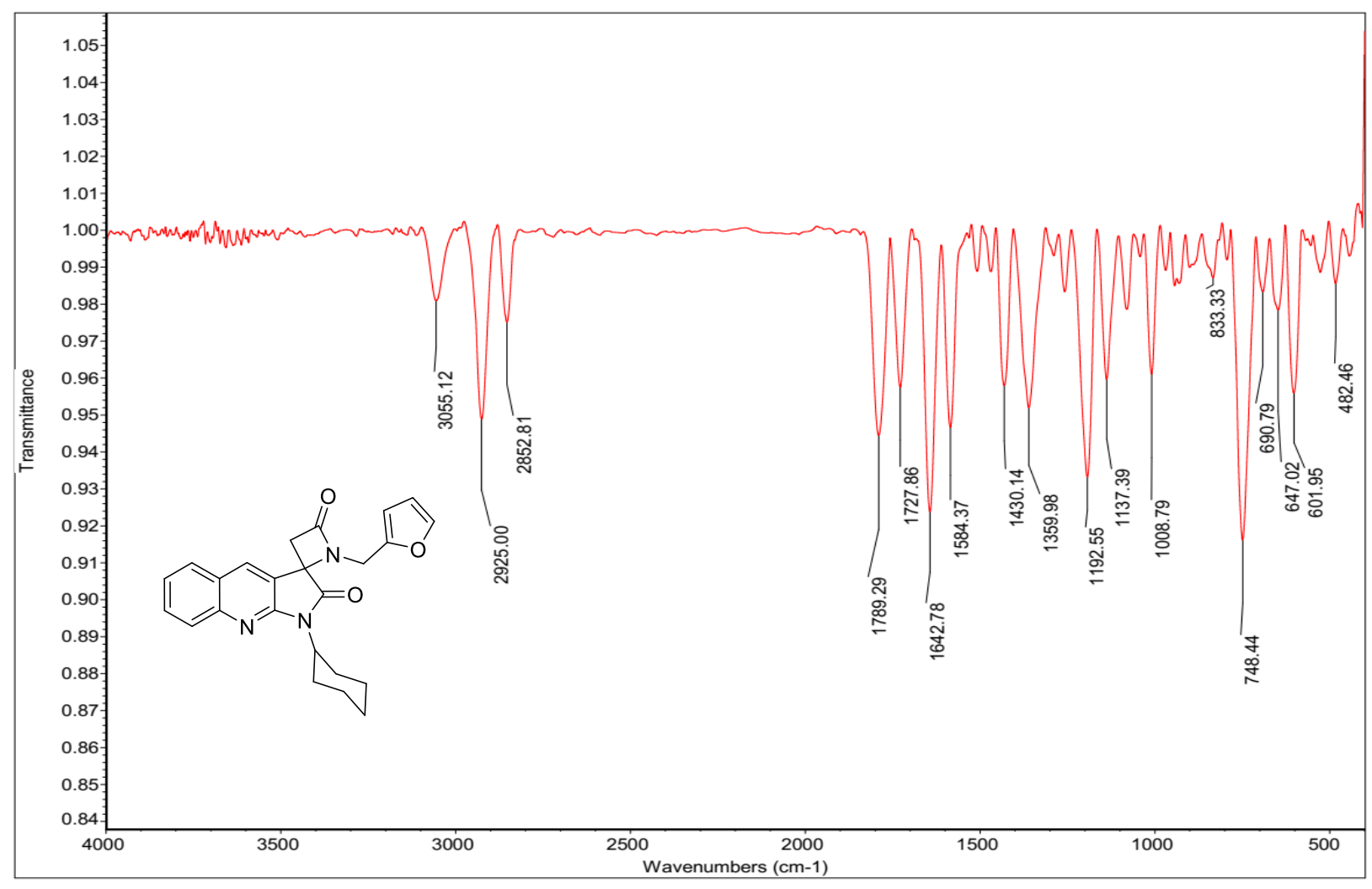

Figure S101: IR of compound $\mathbf{6 c}\left(\mathrm{KBr}, \mathrm{cm}^{-1}\right)$ 


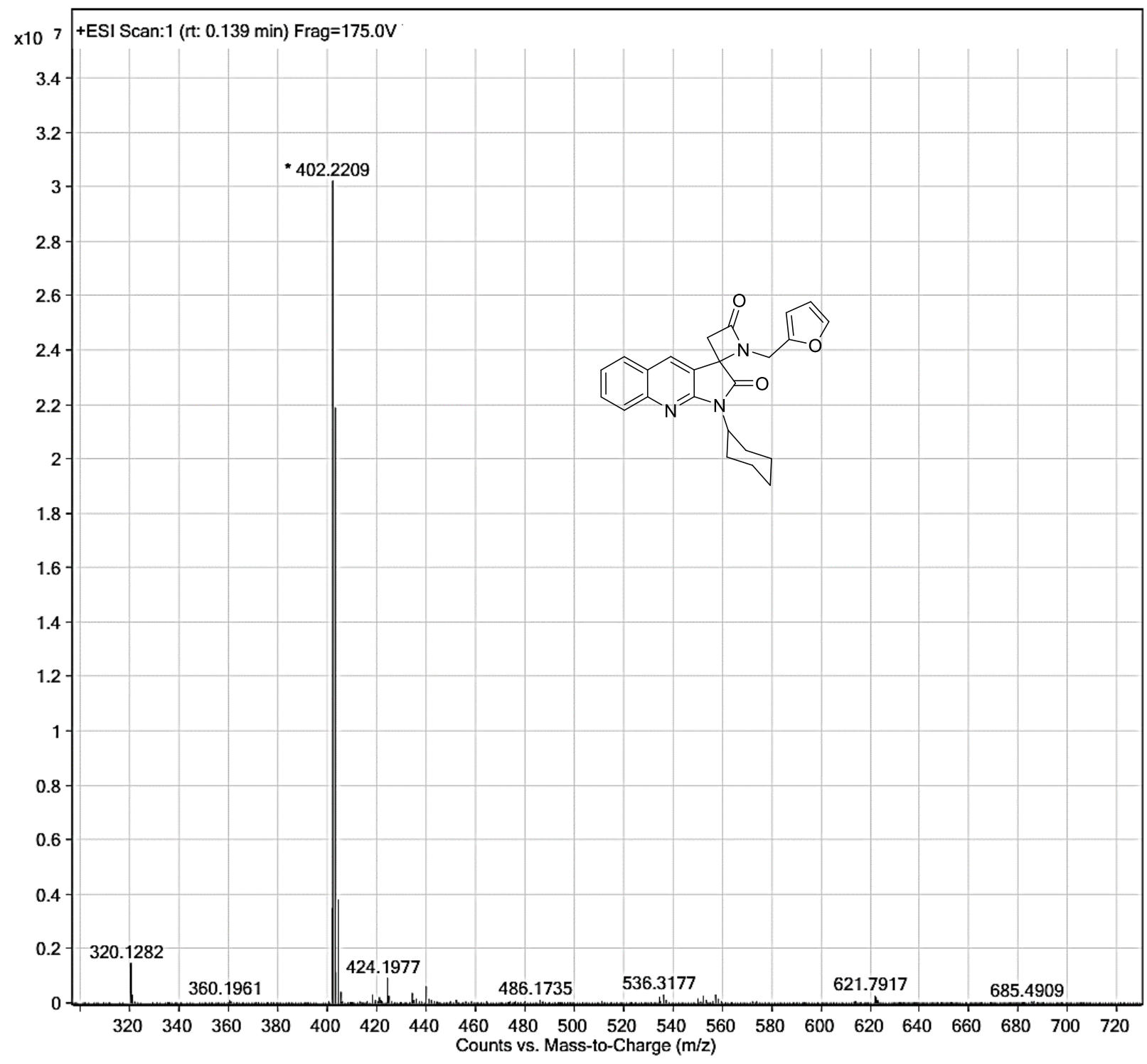

Figure S102: HRMS-ESI of $6 \mathbf{c}$ with formula $\mathrm{C}_{24} \mathrm{H}_{23} \mathrm{~N}_{3} \mathrm{O}_{3}$ and $[\mathrm{M}+\mathrm{H}]^{+} 402.2220$ 

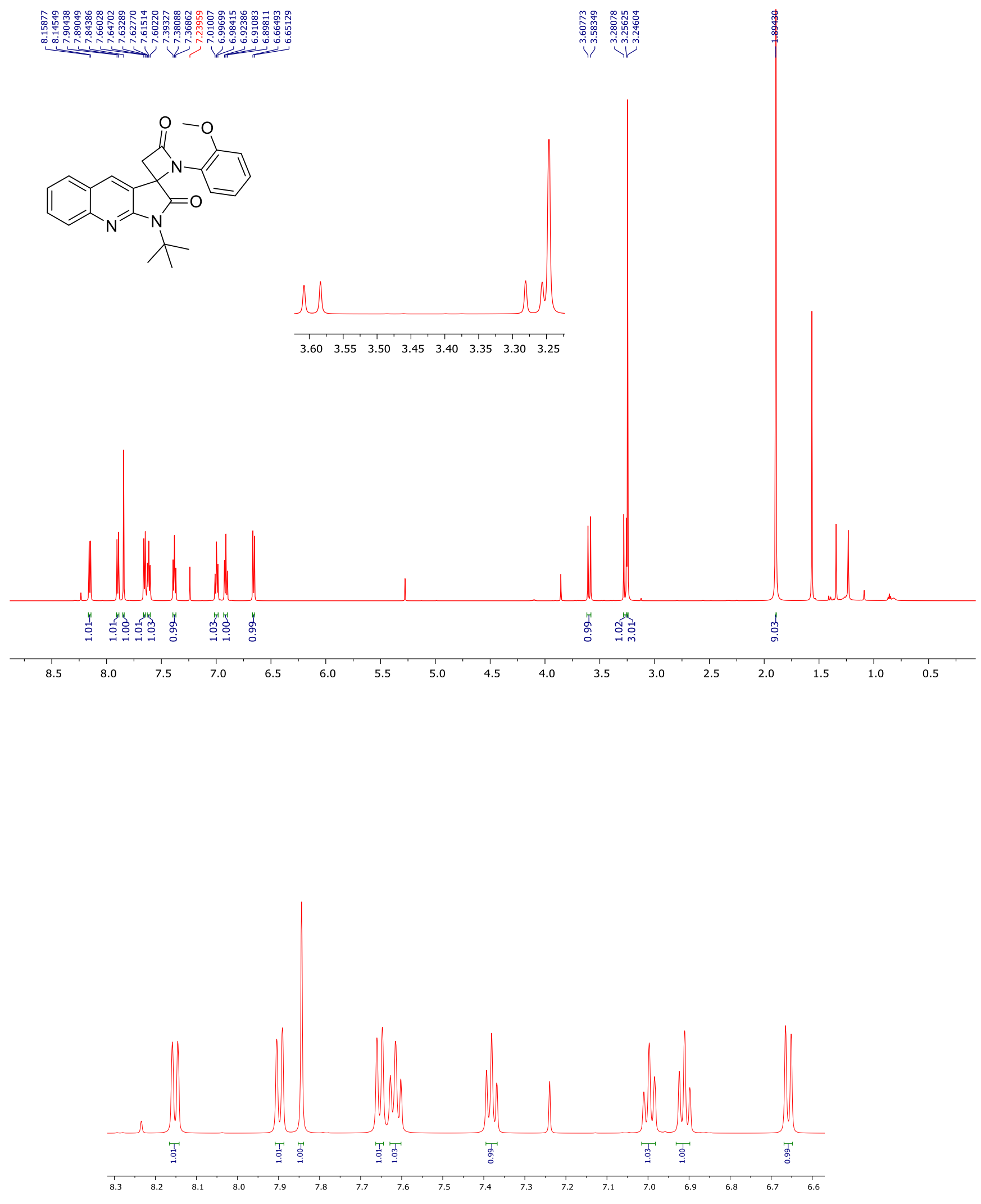

Figure S103: ${ }^{1} \mathrm{H}-\mathrm{NMR}$ of compound $\mathbf{6 d}\left(600 \mathrm{MHz}, \mathrm{CDCl}_{3}\right)$ 


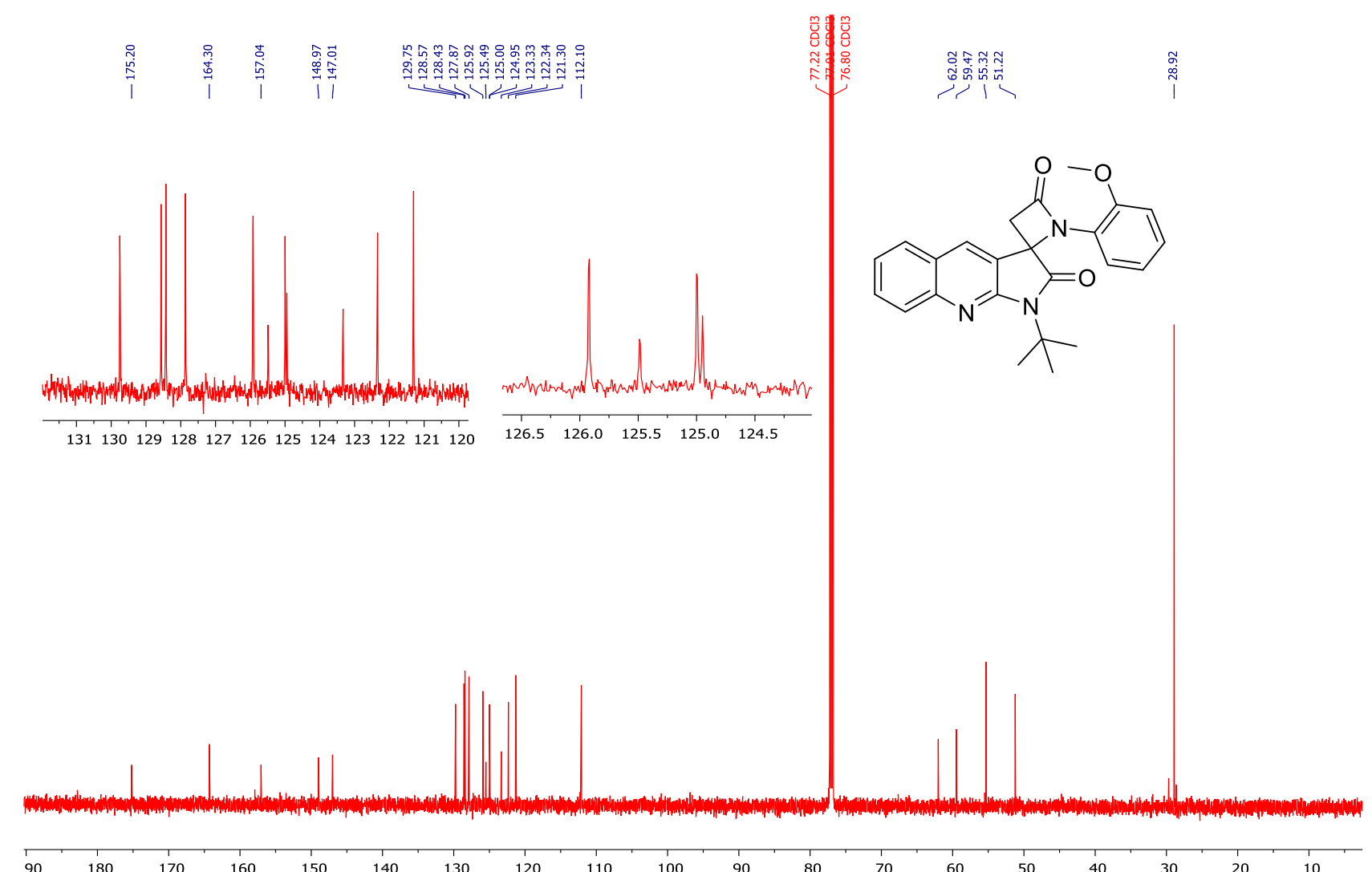

Figure S104: ${ }^{13} \mathrm{C}-\mathrm{NMR}$ of compound $\mathbf{6 d}\left(150 \mathrm{MHz}, \mathrm{CDCl}_{3}\right)$

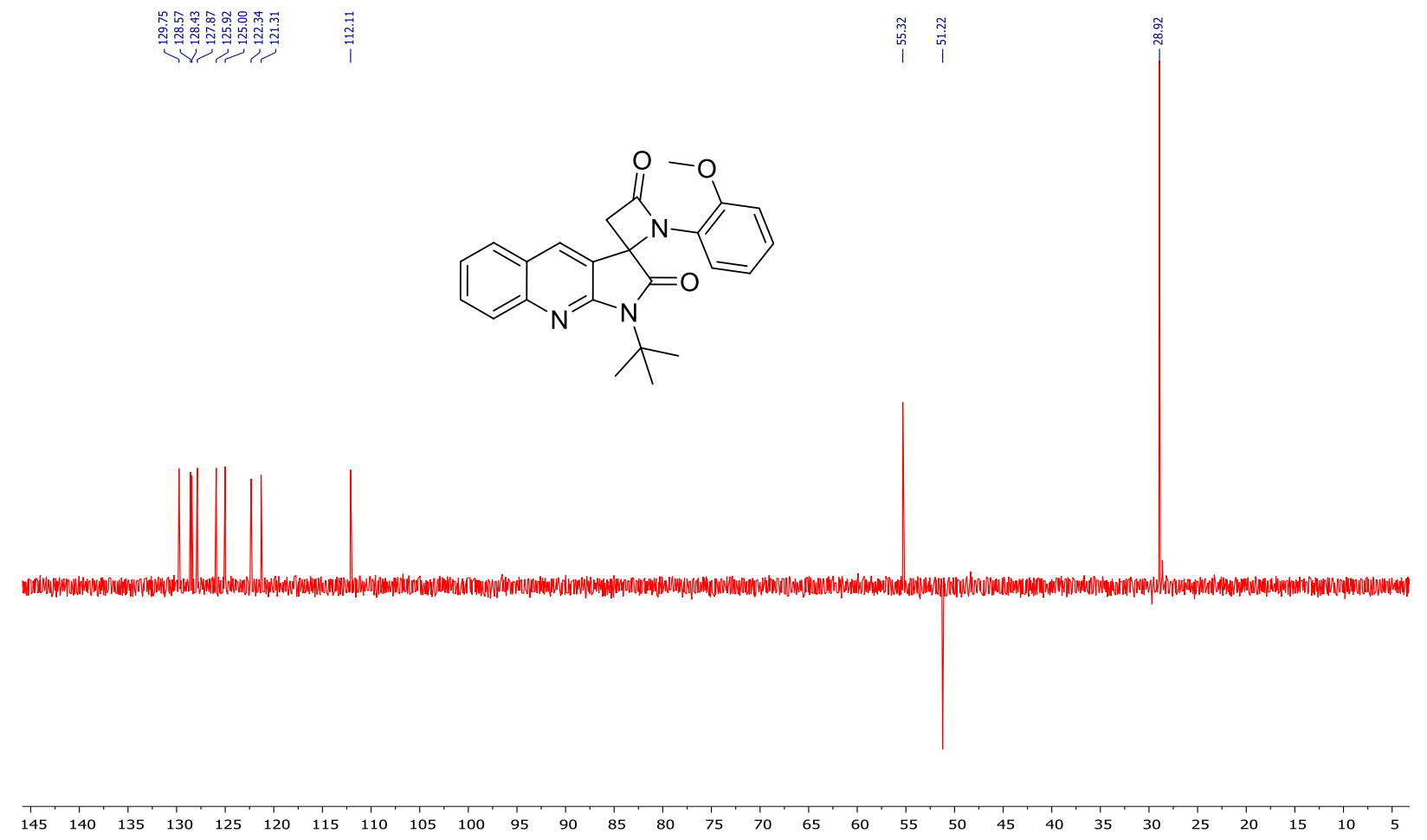

Figure S105: ${ }^{13} \mathrm{C}-\mathrm{NMR}$ (DEPT 135) of compound 6d (150 MHz, $\left.\mathrm{CDCl}_{3}\right)$ 


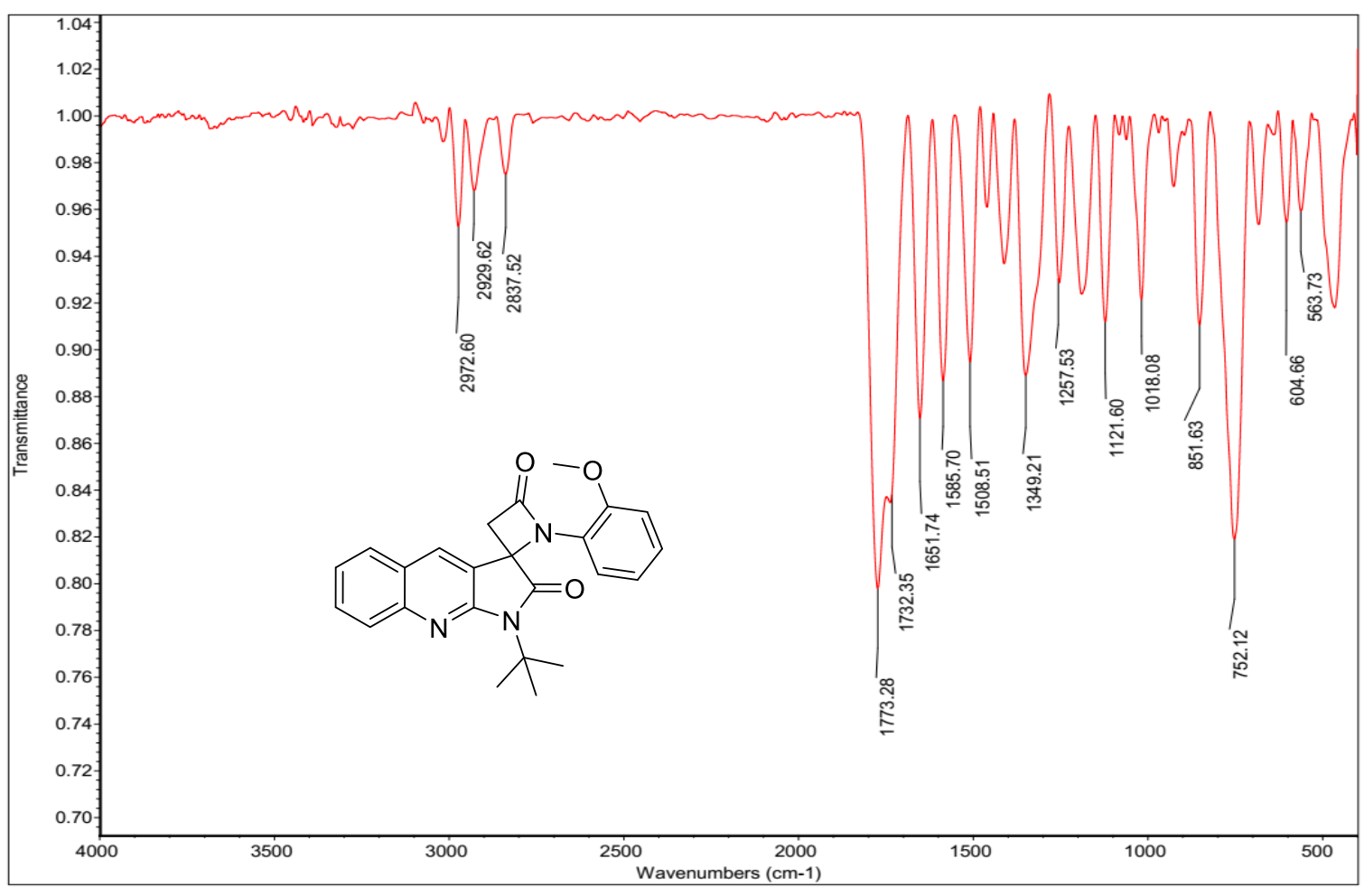

S106: IR of compound $\mathbf{6 d}\left(\mathrm{KBr}, \mathrm{cm}^{-1}\right)$

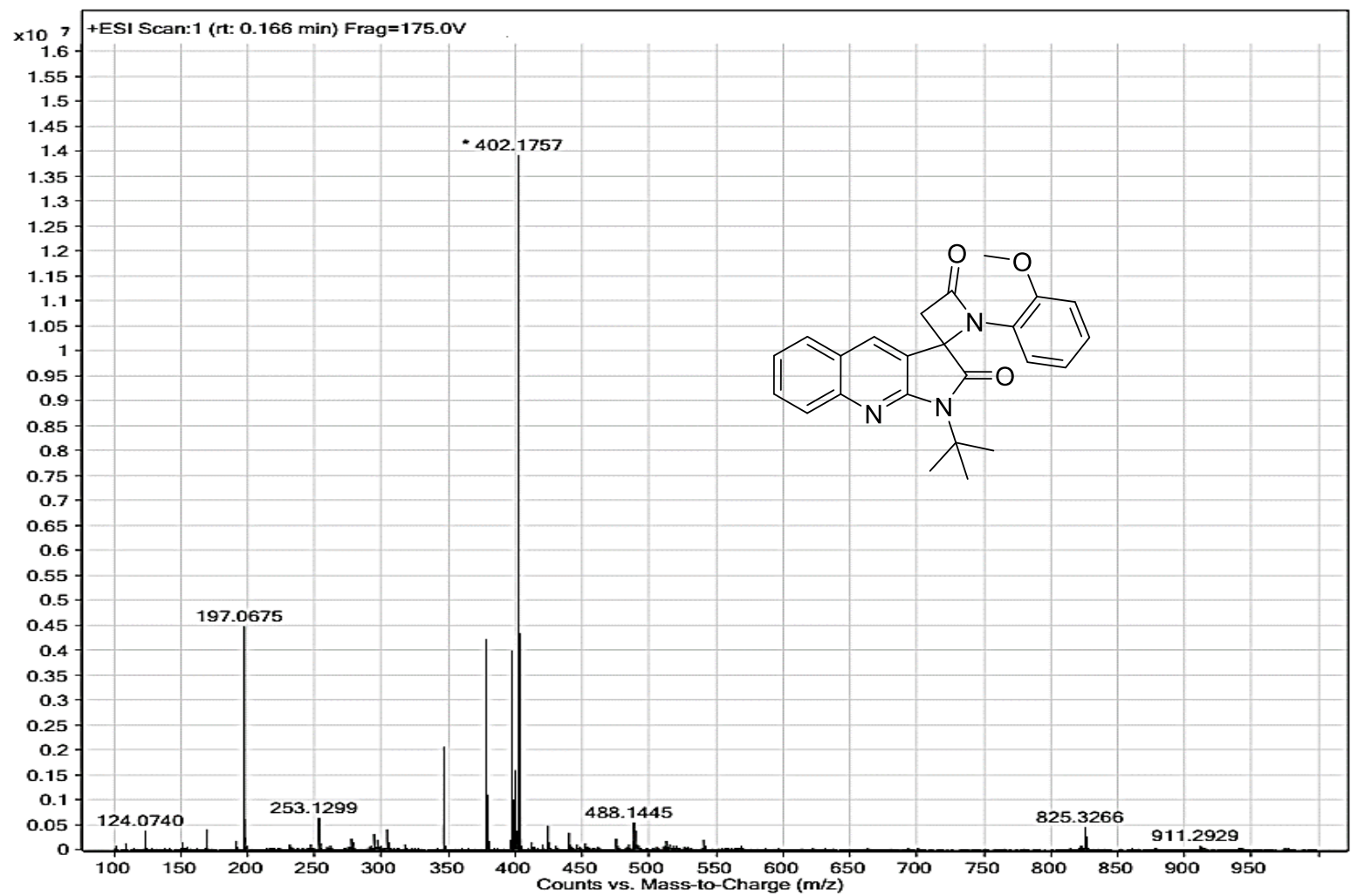

Figure S107: HRMS-ESI of $\mathbf{6 d}$ with formula $\mathrm{C}_{24} \mathrm{H}_{23} \mathrm{~N}_{3} \mathrm{O}_{3}$ and $[\mathrm{M}+\mathrm{H}]^{+} 402.1748$, and [2M+Na] 825.3275 . 

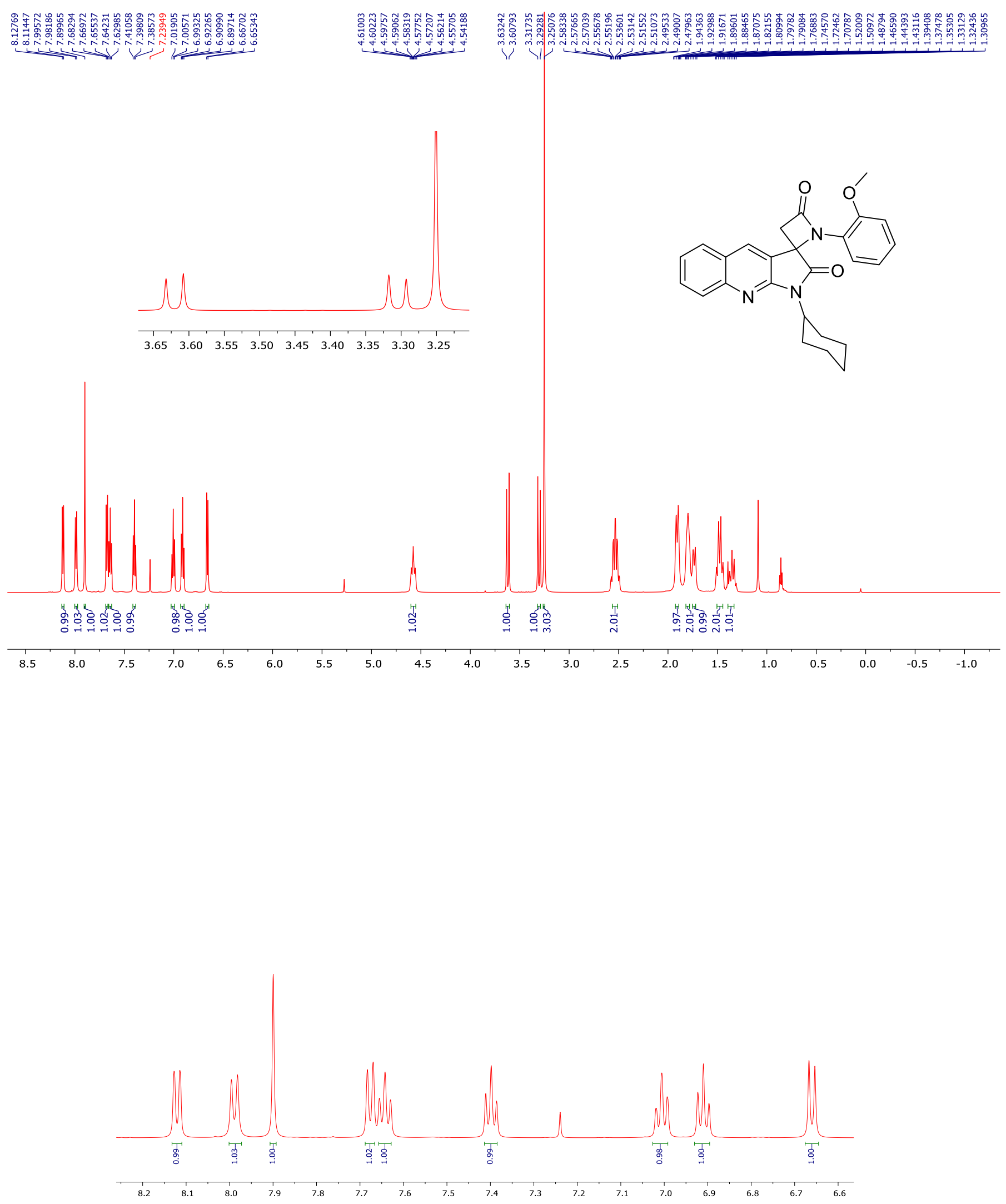

Figure S108: ${ }^{1} \mathrm{H}-\mathrm{NMR}$ of compound $6 \mathbf{e}\left(600 \mathrm{MHz}, \mathrm{CDCl}_{3}\right)$ 


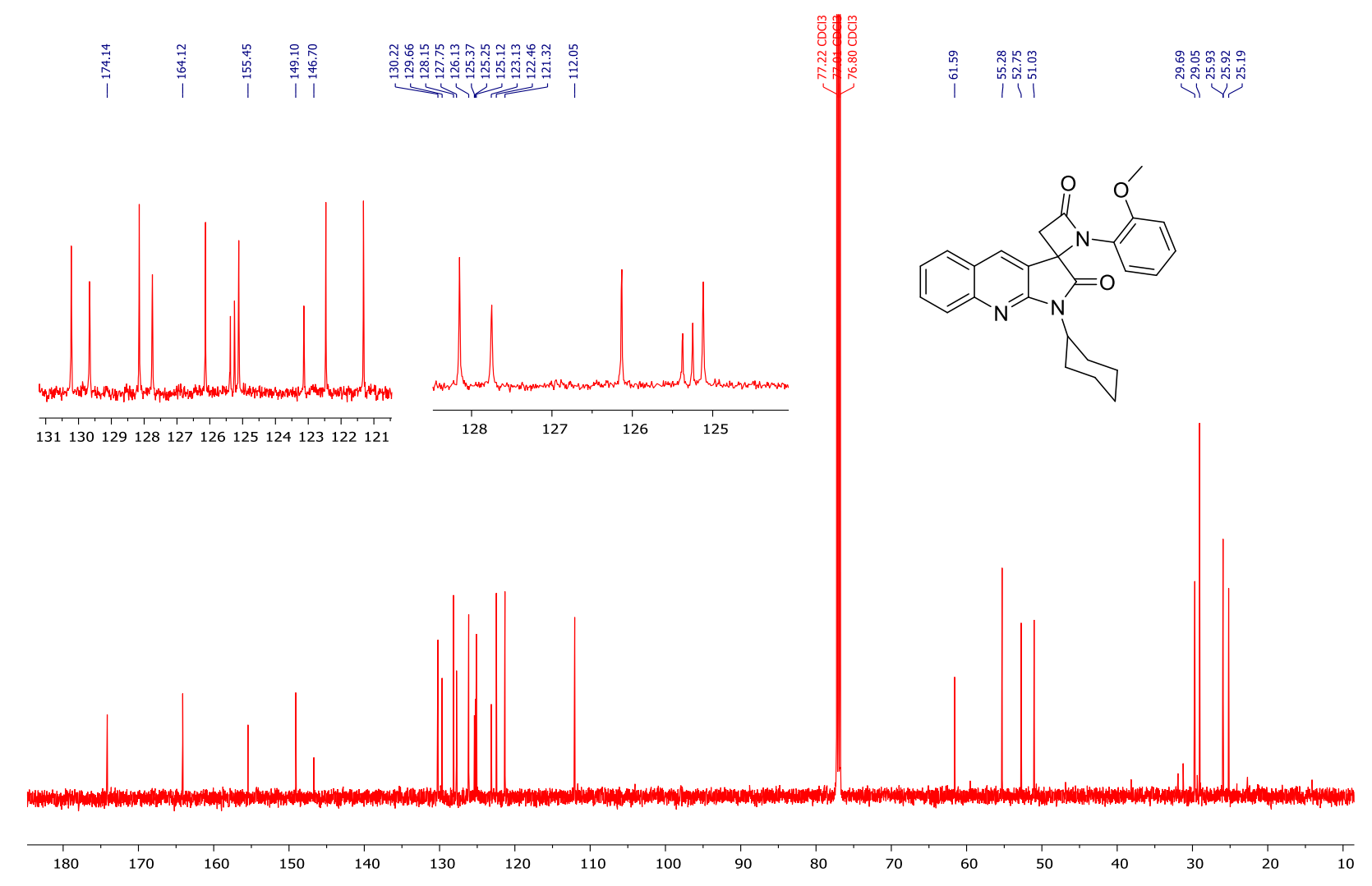

Figure S109: ${ }^{13} \mathrm{C}-\mathrm{NMR}$ of compound $6 \mathbf{e}\left(150 \mathrm{MHz}, \mathrm{CDCl}_{3}\right)$
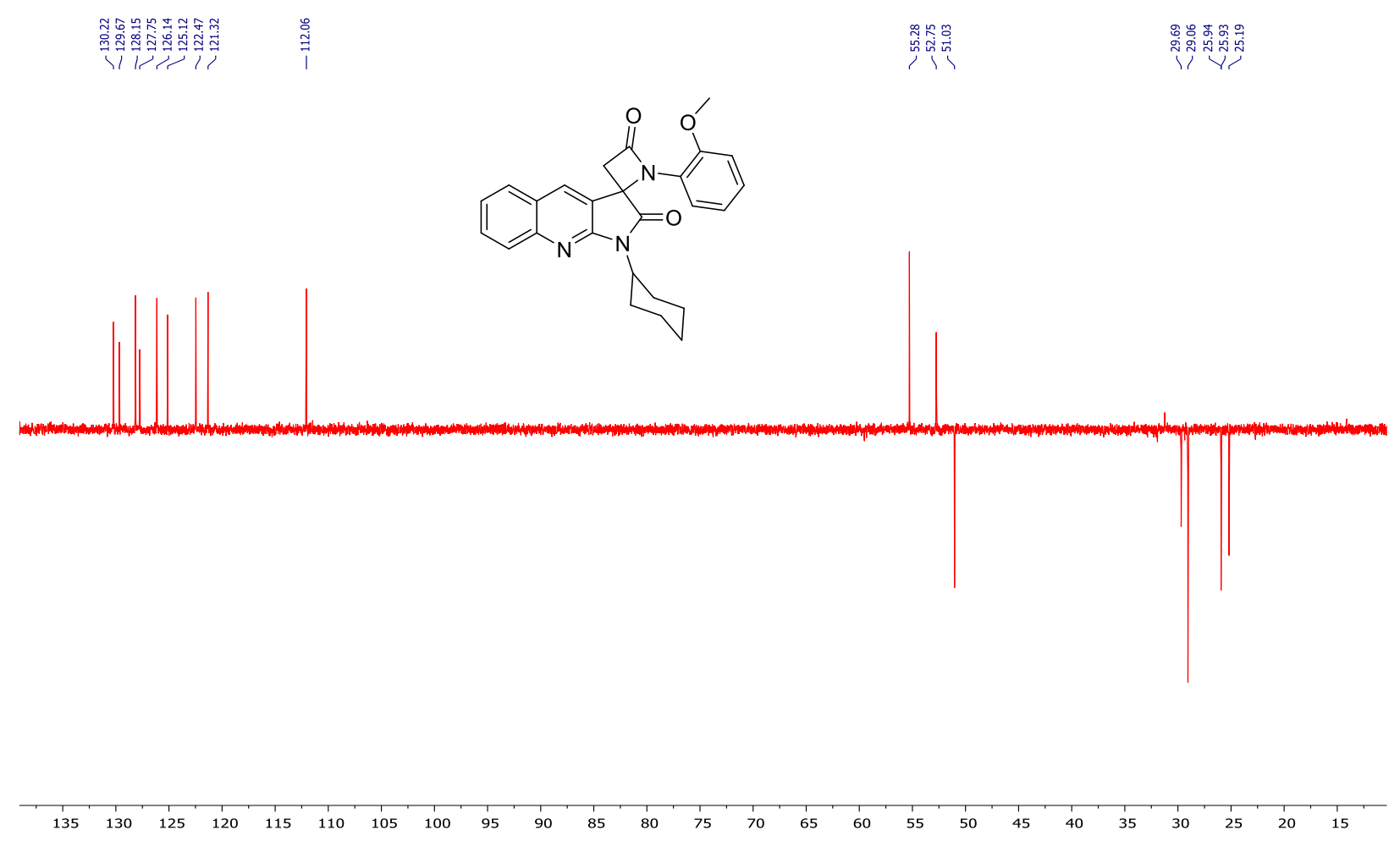

Figure S110: ${ }^{13} \mathrm{C}-\mathrm{NMR}$ (DEPT 135) of compound 6e $\left(150 \mathrm{MHz}, \mathrm{CDCl}_{3}\right.$ ) 


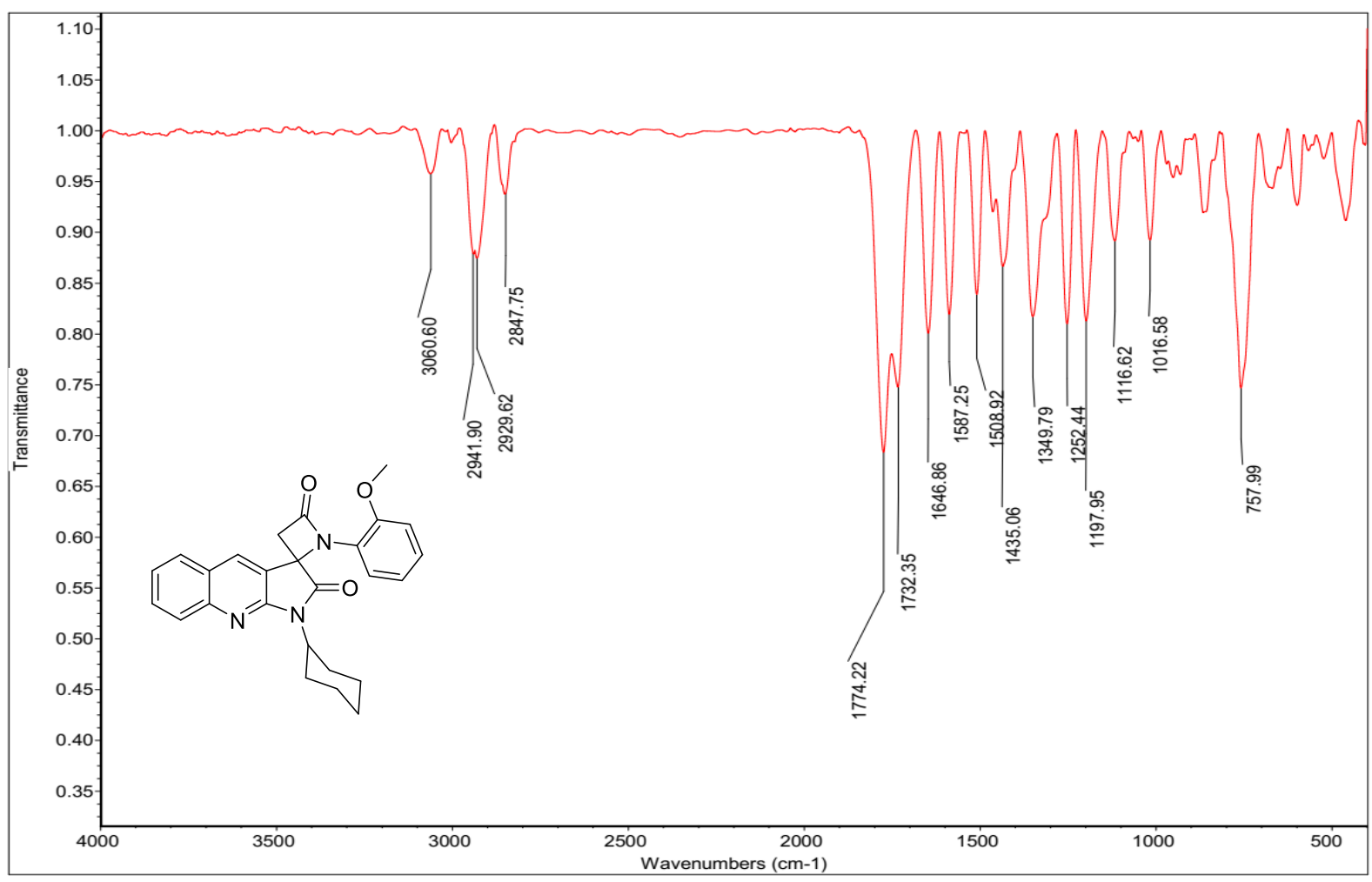

Figure S111: IR of compound $6 \mathrm{e}\left(\mathrm{KBr}, \mathrm{cm}^{-1}\right)$

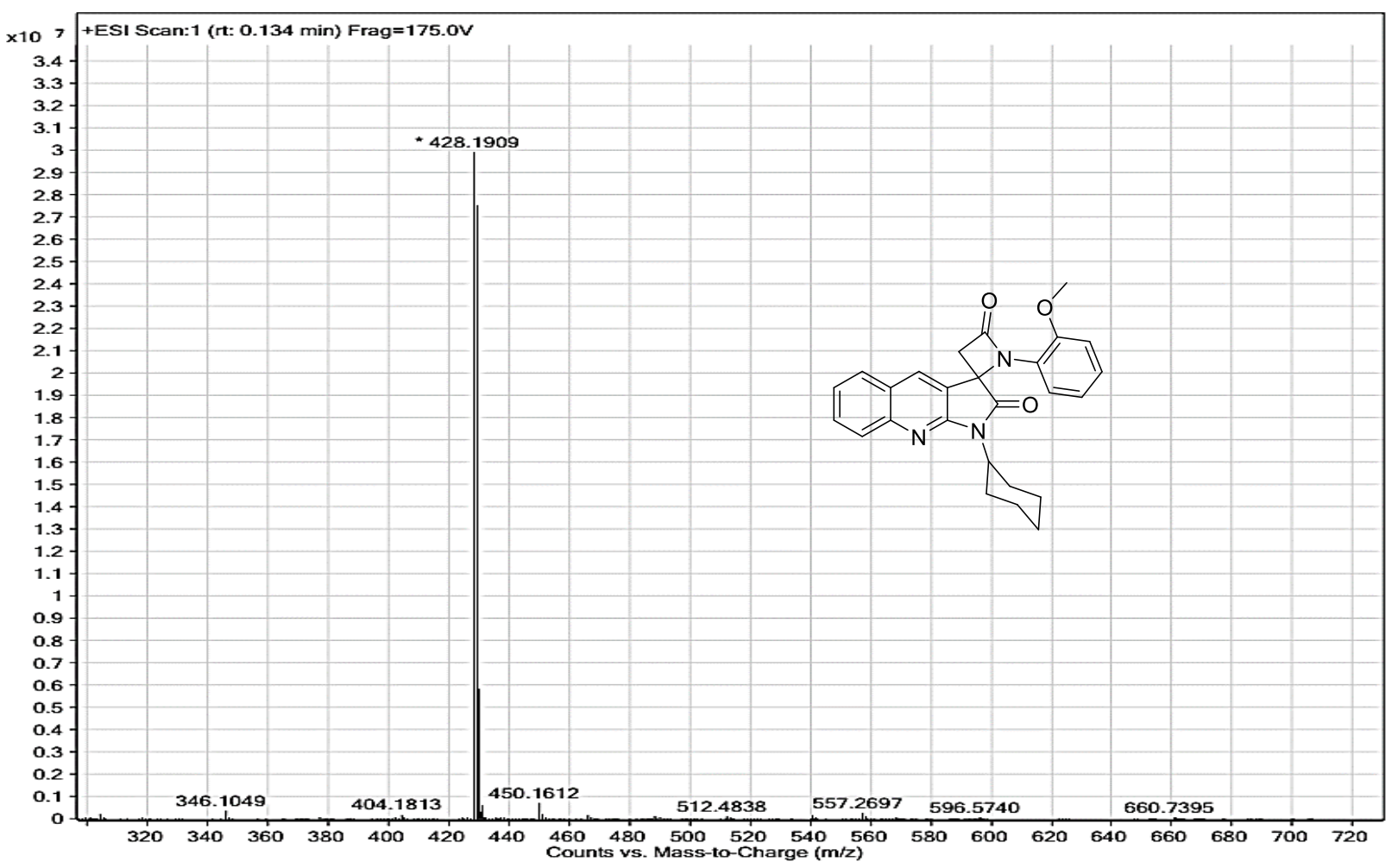

Figure S112: HRMS-ESI of $6 \mathbf{e}$ with formula $\mathrm{C}_{26} \mathrm{H}_{25} \mathrm{~N}_{3} \mathrm{O}_{3}$ and $[\mathrm{M}+\mathrm{H}]^{+} 428.1920$, and $[\mathrm{M}+\mathrm{Na}]^{+}$ 450.1621 . 

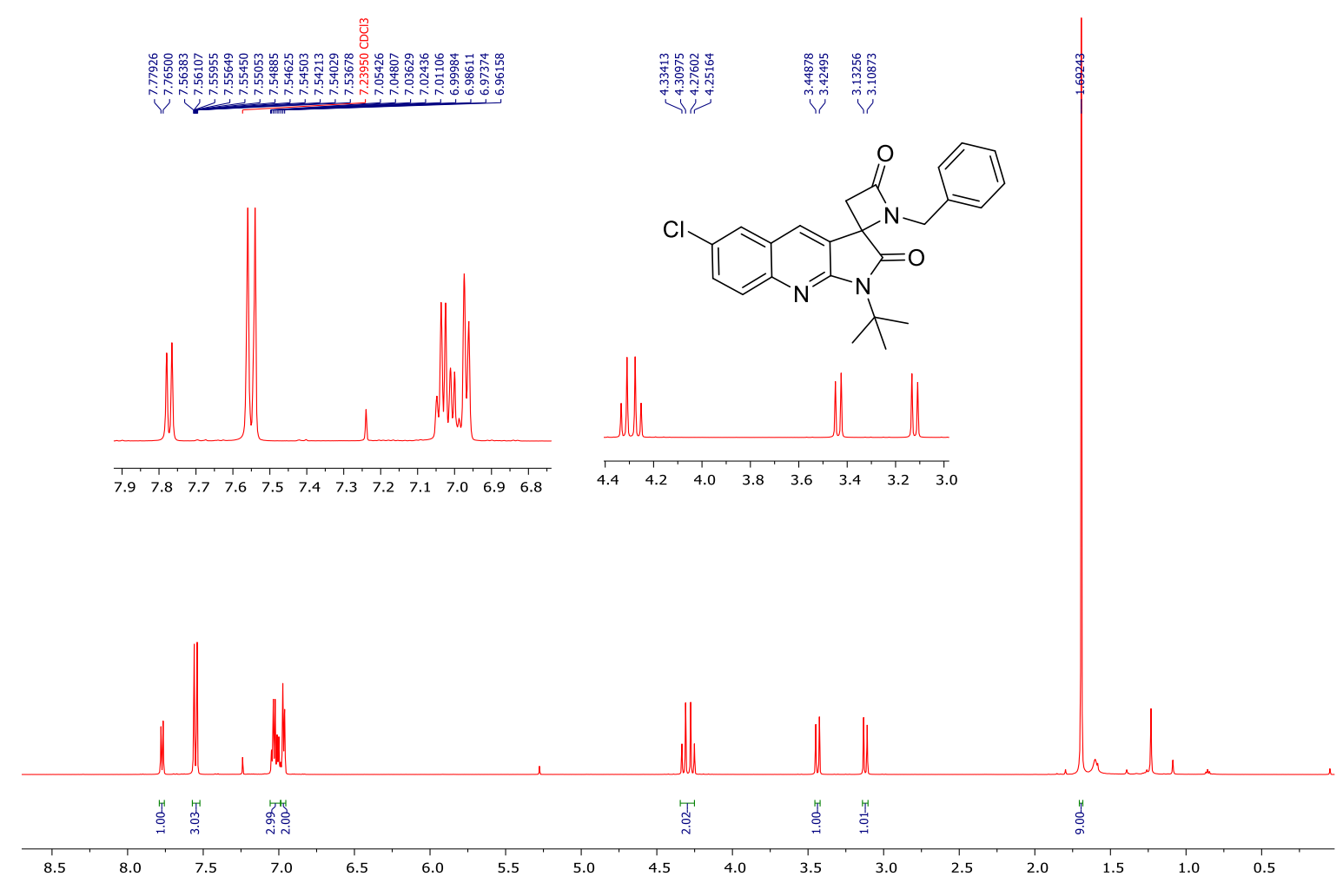

Figure S113: ${ }^{1} \mathrm{H}-\mathrm{NMR}$ of compound $\mathbf{6 f}\left(600 \mathrm{MHz}, \mathrm{CDCl}_{3}\right)$

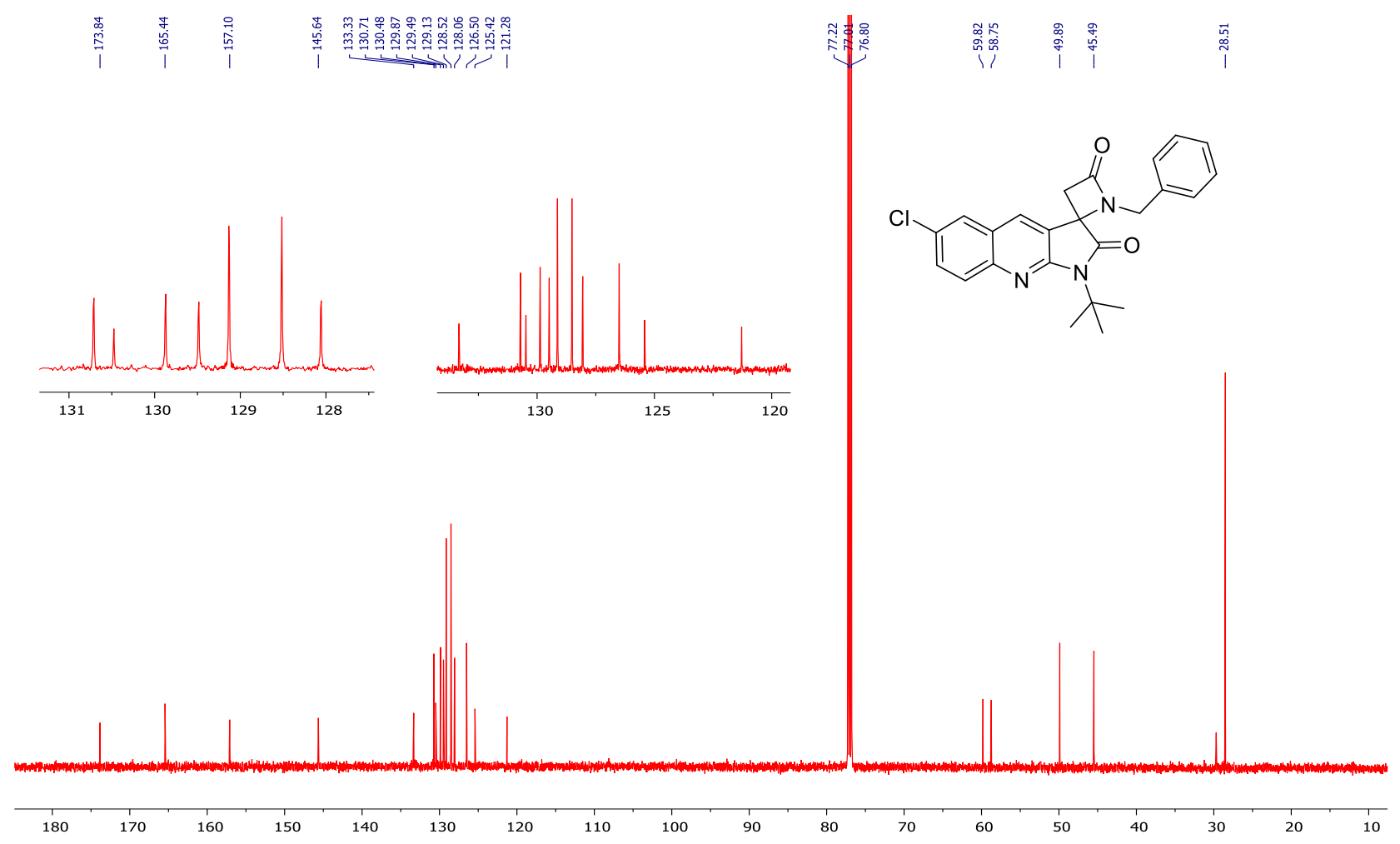

Figure S114: ${ }^{13} \mathrm{C}-\mathrm{NMR}$ of compound $\mathbf{6 f}\left(150 \mathrm{MHz}, \mathrm{CDCl}_{3}\right)$ 


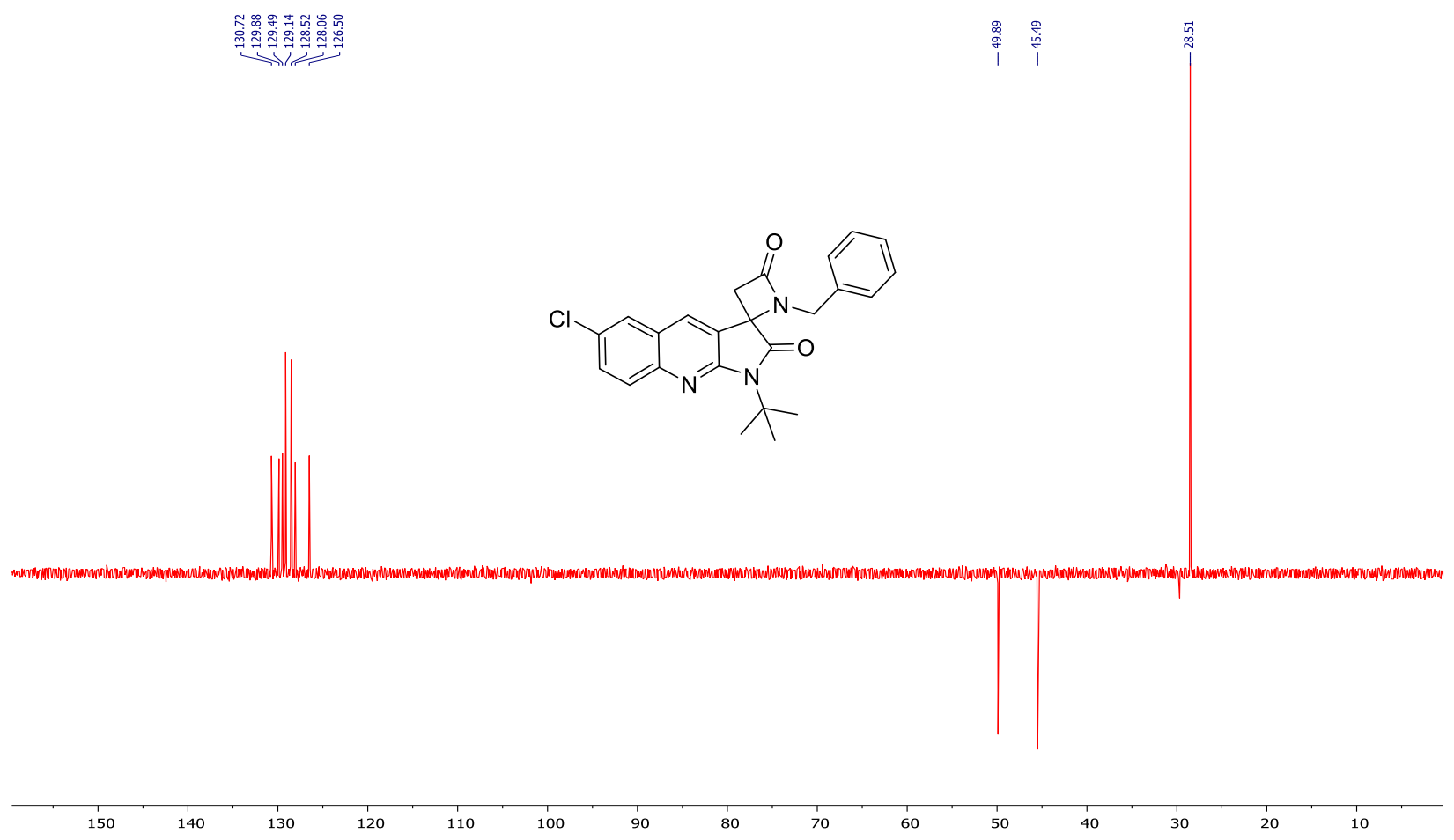

Figure S115: ${ }^{13} \mathrm{C}-\mathrm{NMR}$ (DEPT 135) of compound $\mathbf{6 f}\left(150 \mathrm{MHz}, \mathrm{CDCl}_{3}\right)$

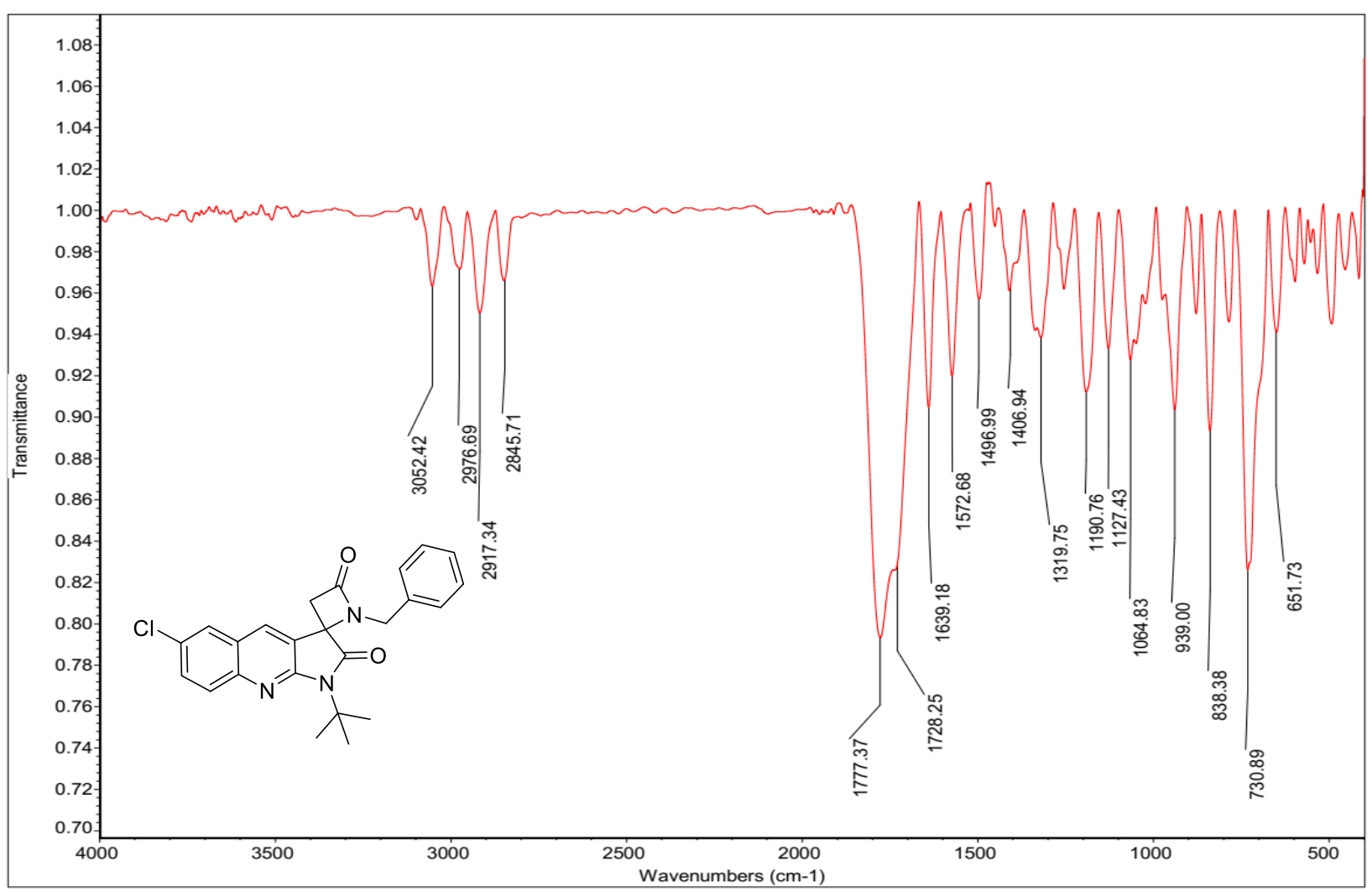

Figure S116: IR of compound $6 \mathbf{f}\left(\mathrm{KBr}, \mathrm{cm}^{-1}\right)$ 


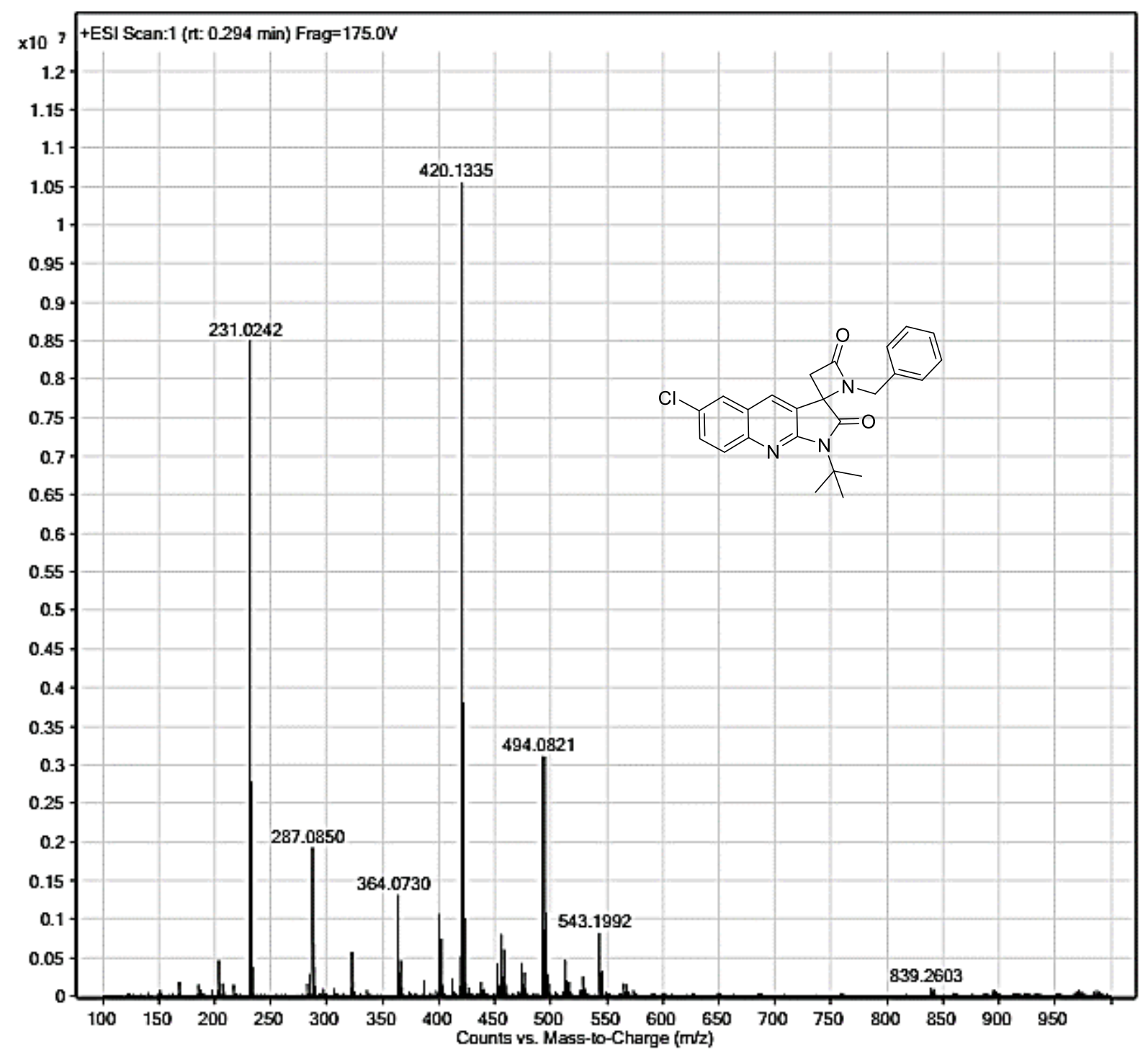

Figure S117: HRMS-ESI of 6 f with formula $\mathrm{C}_{24} \mathrm{H}_{22} \mathrm{ClN}_{3} \mathrm{O}_{2}$ and $[\mathrm{M}+\mathrm{H}]^{+} 420.1326$, and $[2 \mathrm{M}+\mathrm{H}]^{+} 839.2595$ 

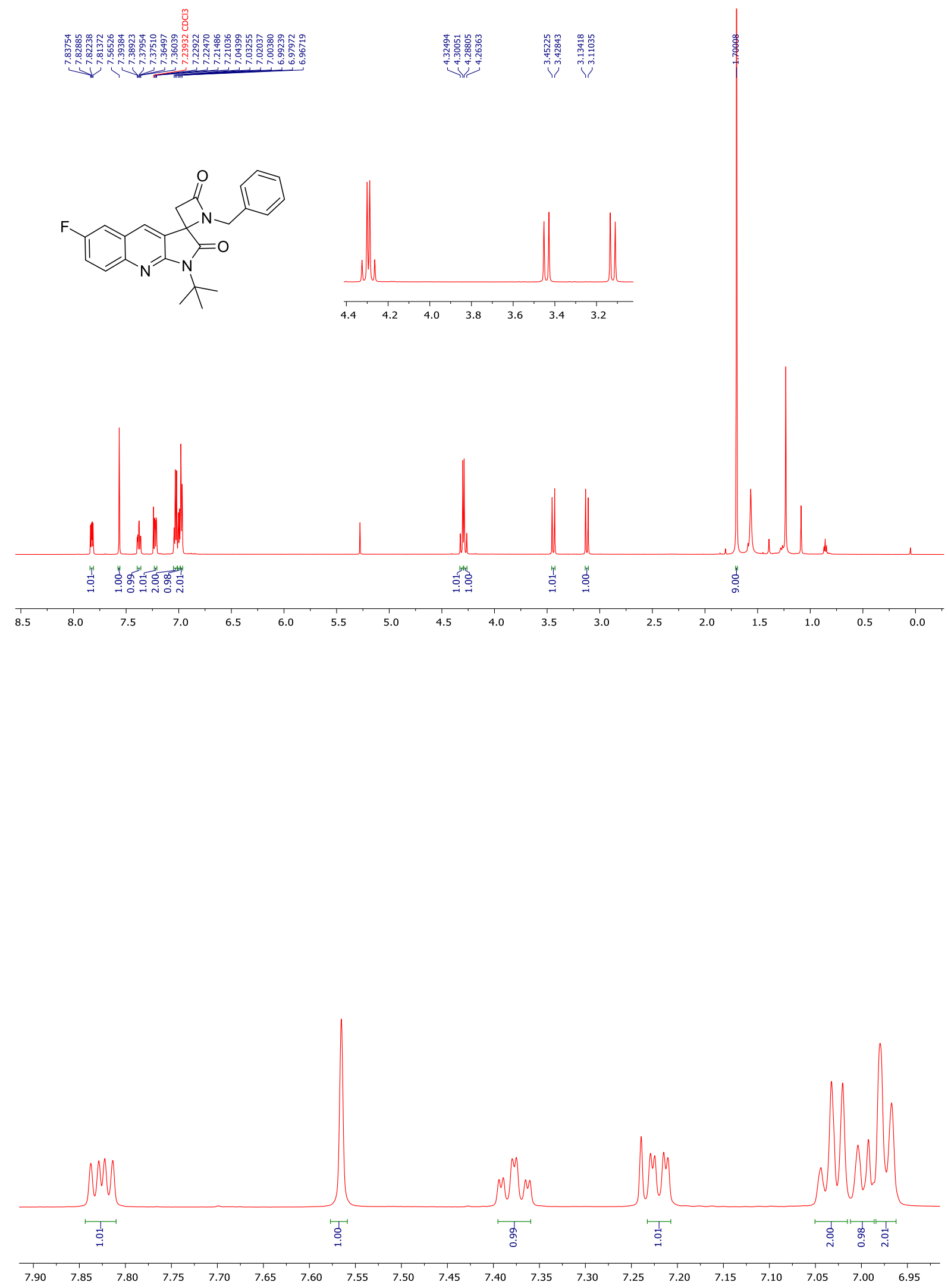

Figure S118: ${ }^{1} \mathrm{H}-\mathrm{NMR}$ of compound $\mathbf{6 g}\left(600 \mathrm{MHz}, \mathrm{CDCl}_{3}\right)$ 

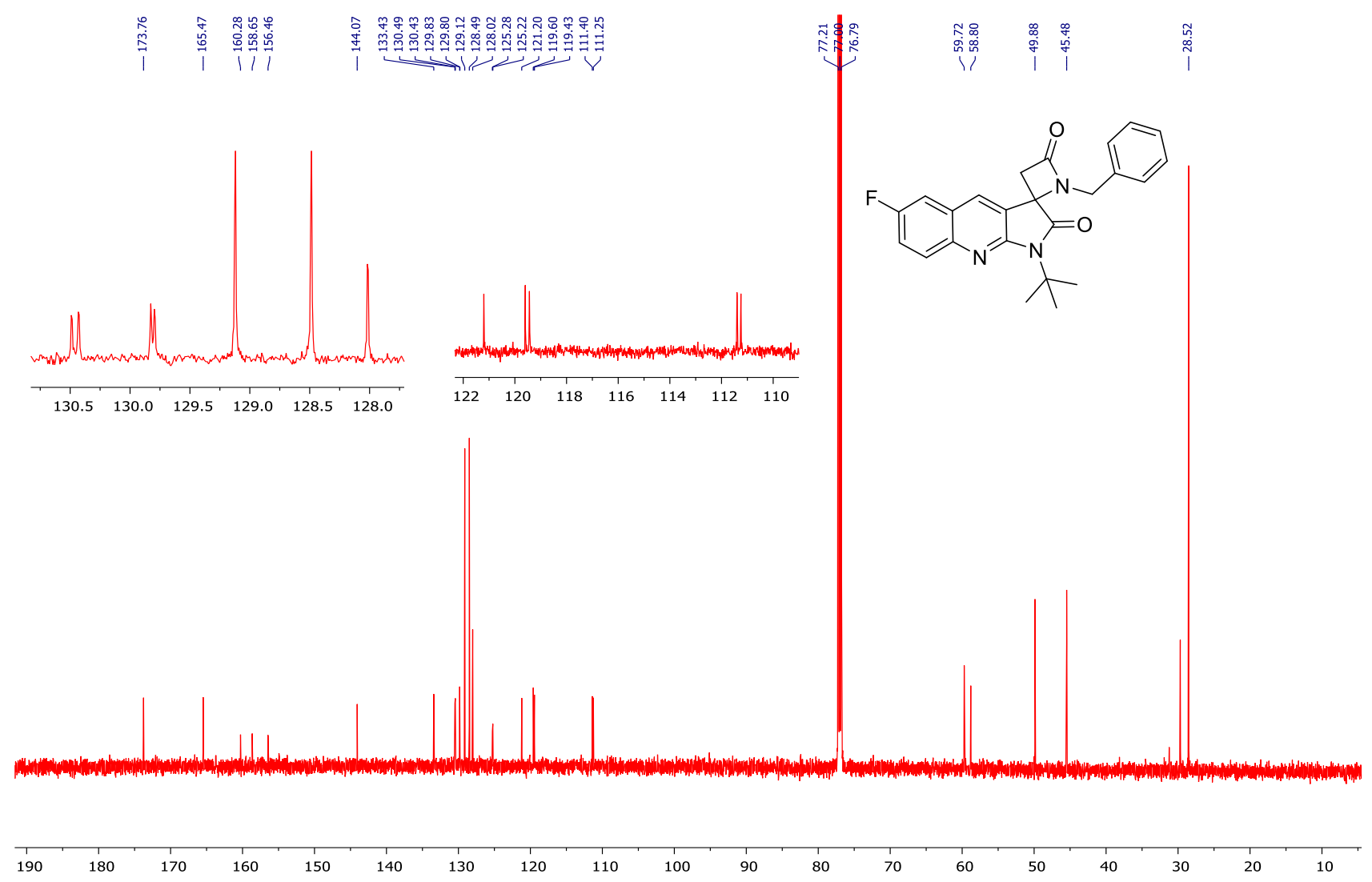

Figure S119: ${ }^{13} \mathrm{C}-\mathrm{NMR}$ of compound $\mathbf{6 g}\left(150 \mathrm{MHz}, \mathrm{CDCl}_{3}\right)$

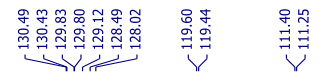

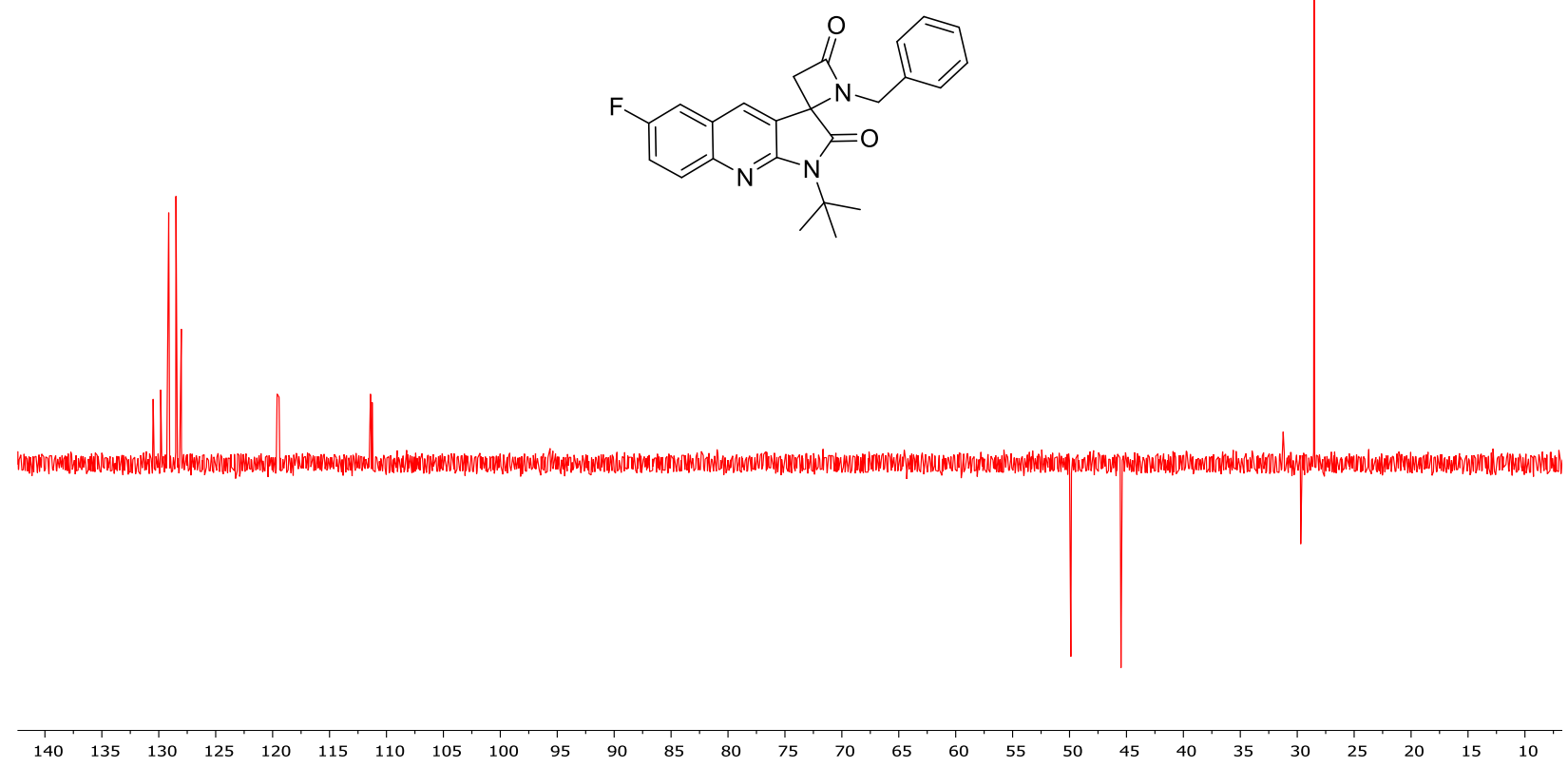

Figure S120: ${ }^{13} \mathrm{C}-\mathrm{NMR}$ (DEPT 135) of compound $6 \mathrm{~g}\left(150 \mathrm{MHz}, \mathrm{CDCl}_{3}\right)$ 


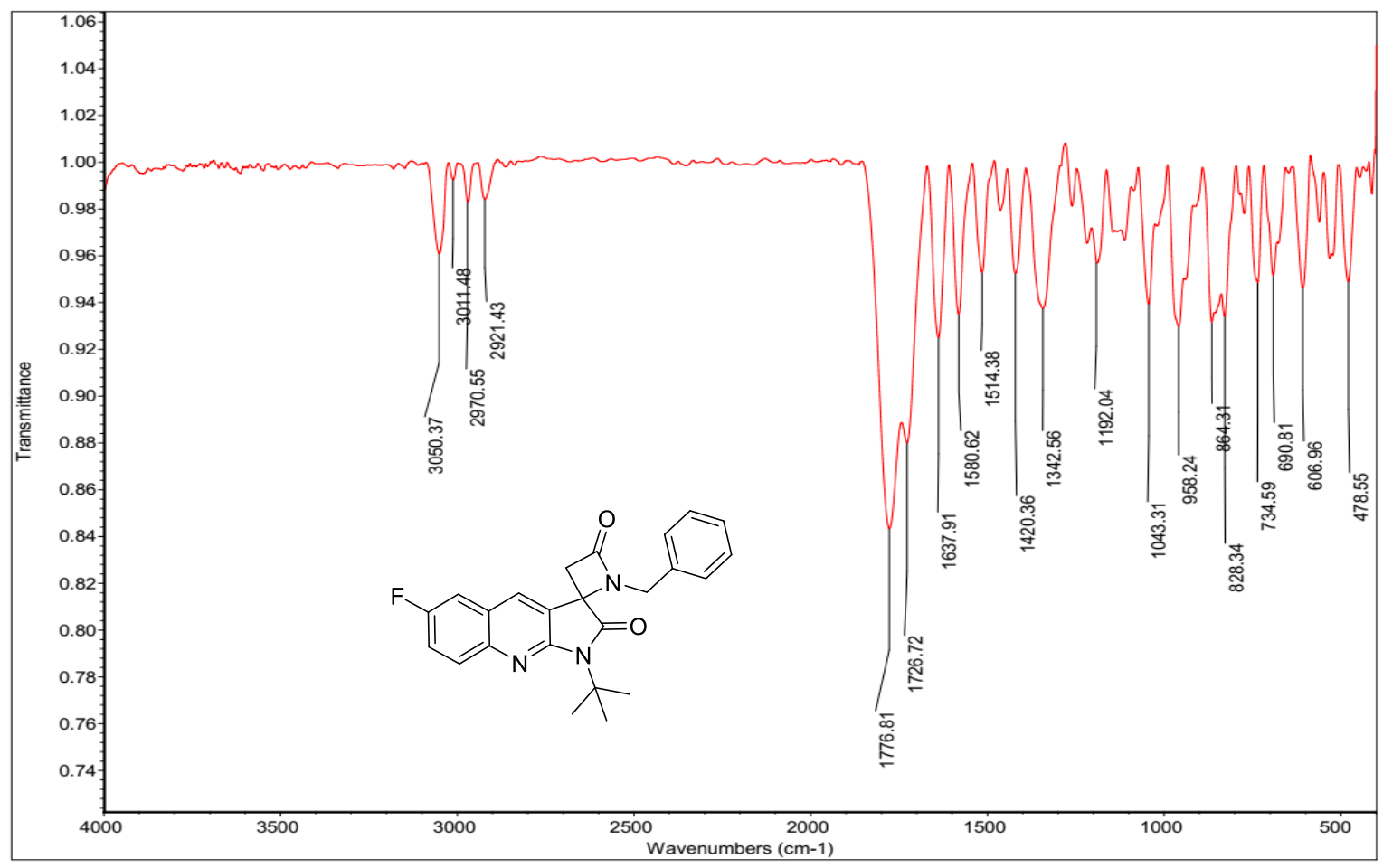

Figure S121: IR of compound $6 \mathrm{~g}\left(\mathrm{KBr}, \mathrm{cm}^{-1}\right)$

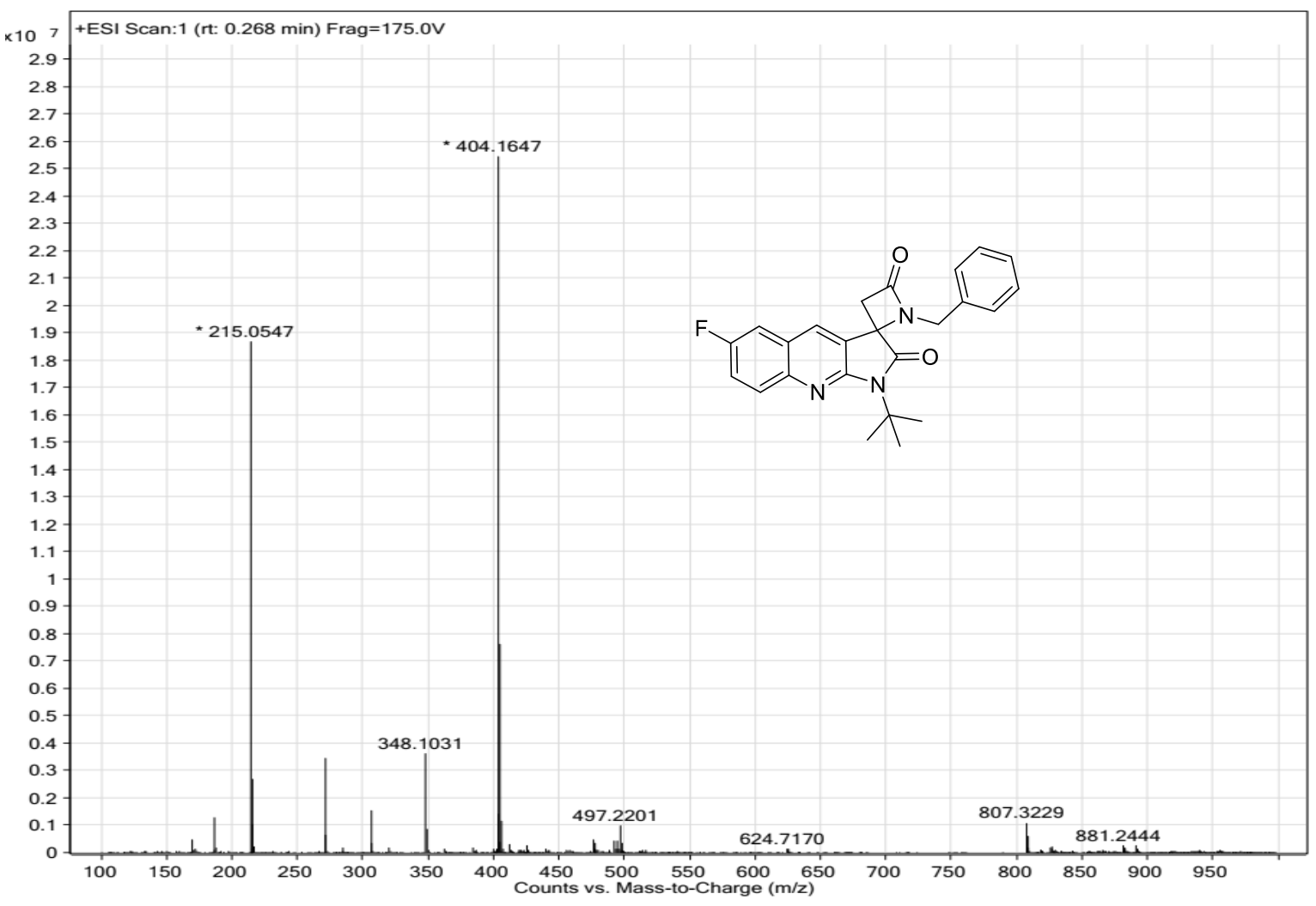

Figure S122: HRMS-ESI of $\mathbf{6 g}$ with formula $\mathrm{C}_{24} \mathrm{H}_{22} \mathrm{FN}_{3} \mathrm{O}_{2}$ and $[\mathrm{M}+\mathrm{H}]^{+} 404.1640$, and $[2 \mathrm{M}+\mathrm{H}]^{+}$ 807.3220 


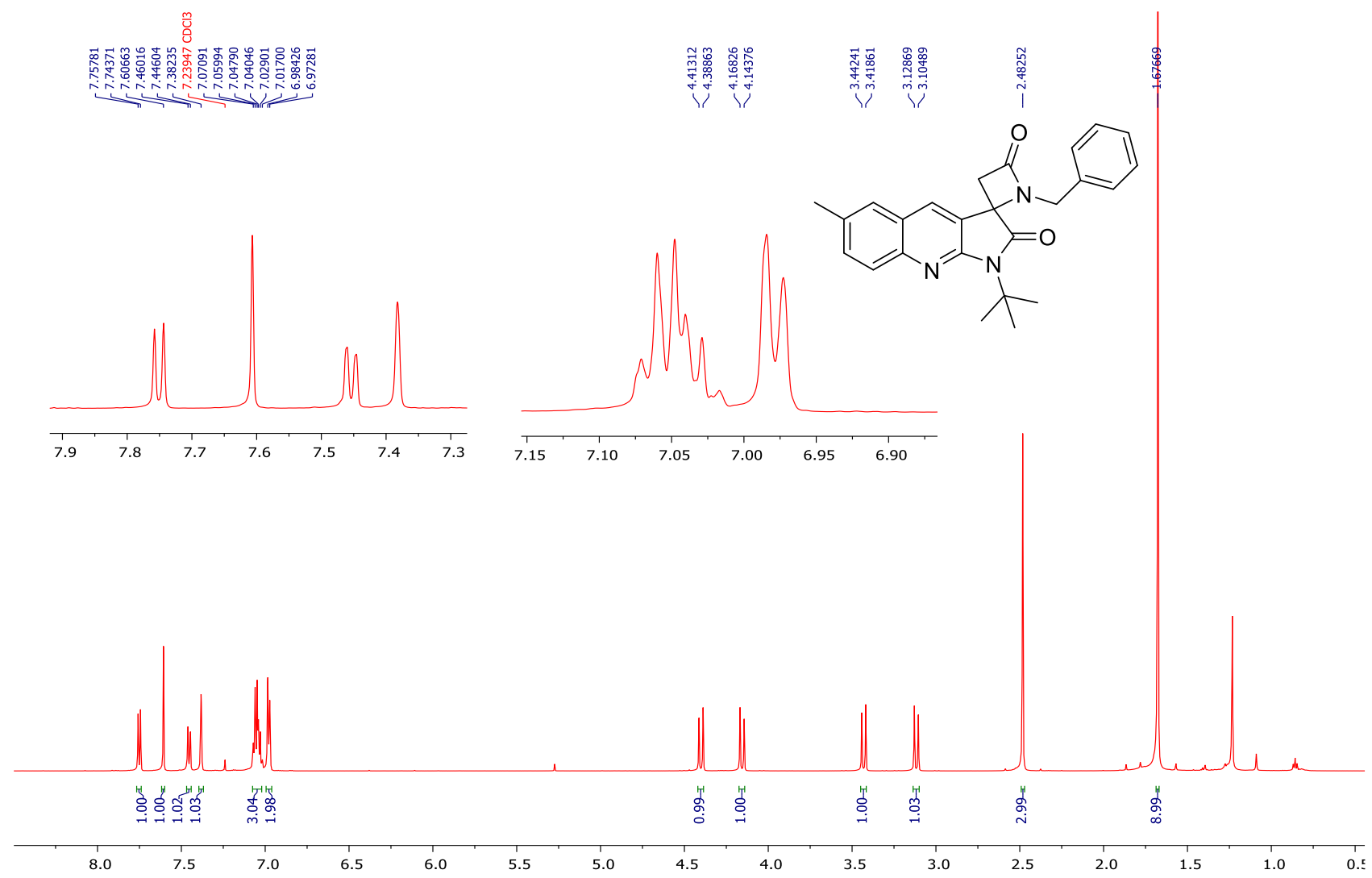

Figure S123: ${ }^{1} \mathrm{H}-\mathrm{NMR}$ of compound $\mathbf{6 h}\left(600 \mathrm{MHz}, \mathrm{CDCl}_{3}\right)$

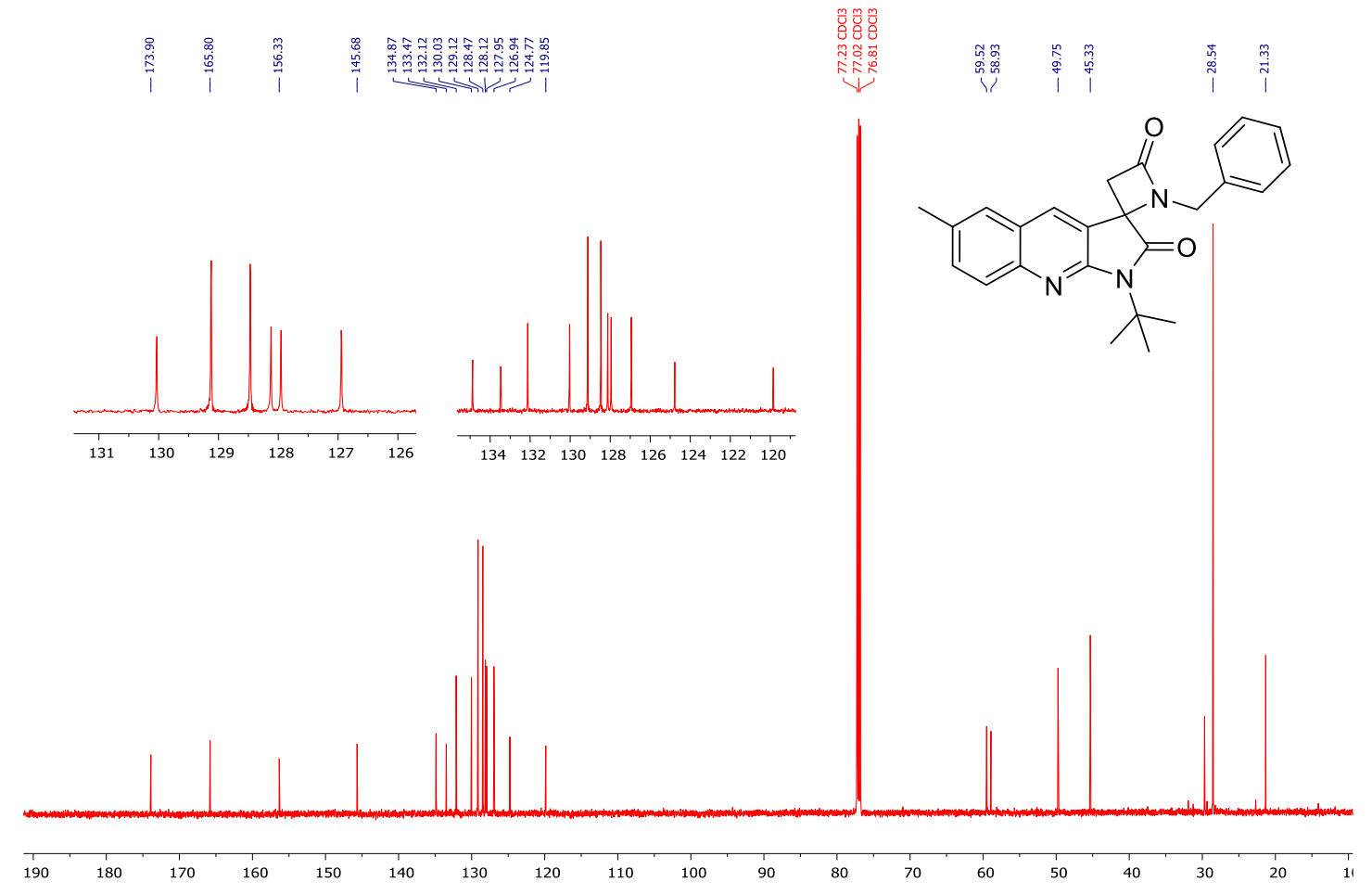

Figure S124: ${ }^{13} \mathrm{C}-\mathrm{NMR}$ of compound $\mathbf{6 h}\left(150 \mathrm{MHz}, \mathrm{CDCl}_{3}\right)$ 


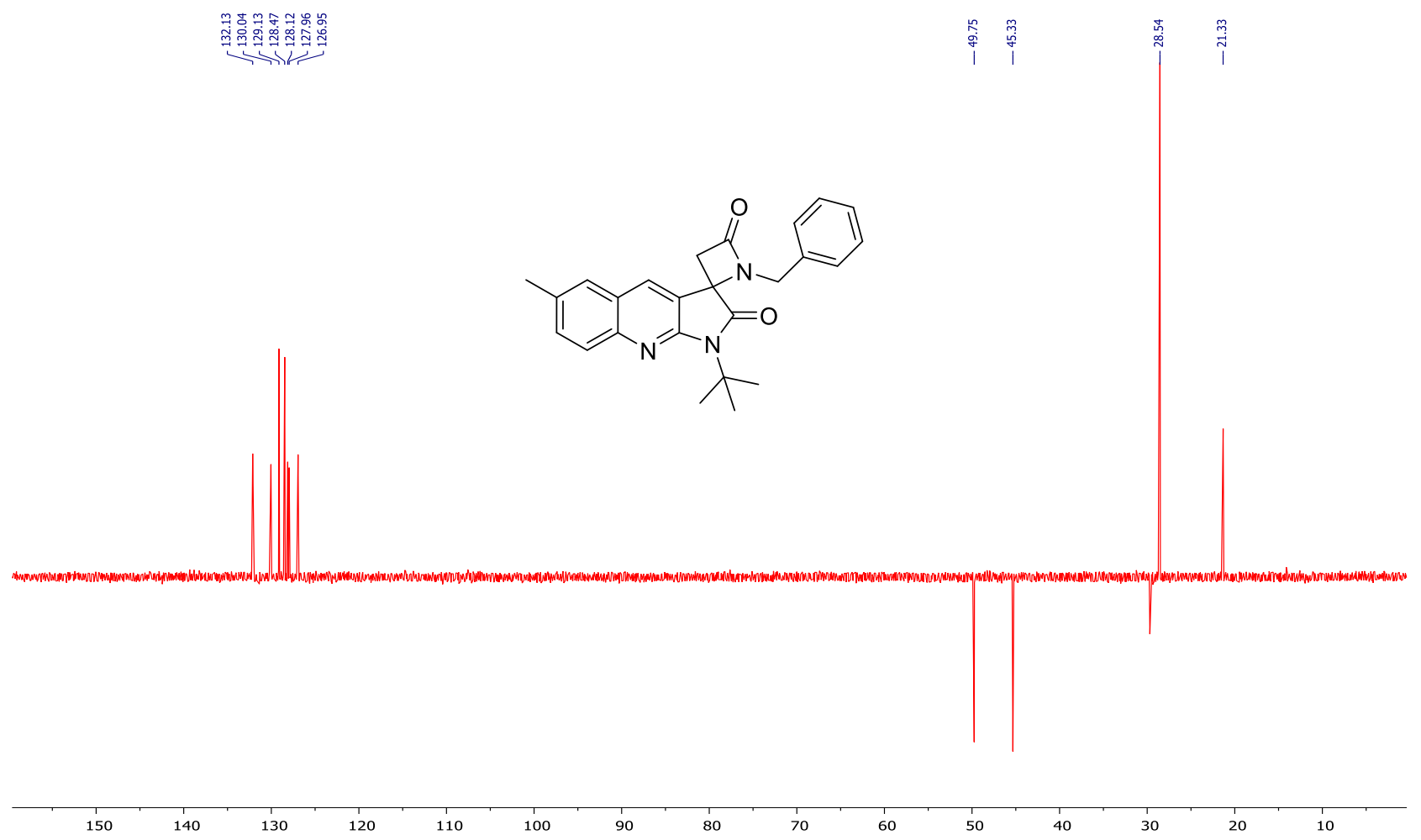

Figure S125: ${ }^{13} \mathrm{C}-\mathrm{NMR}$ (DEPT 135) of compound $\mathbf{6 h}\left(150 \mathrm{MHz}, \mathrm{CDCl}_{3}\right)$

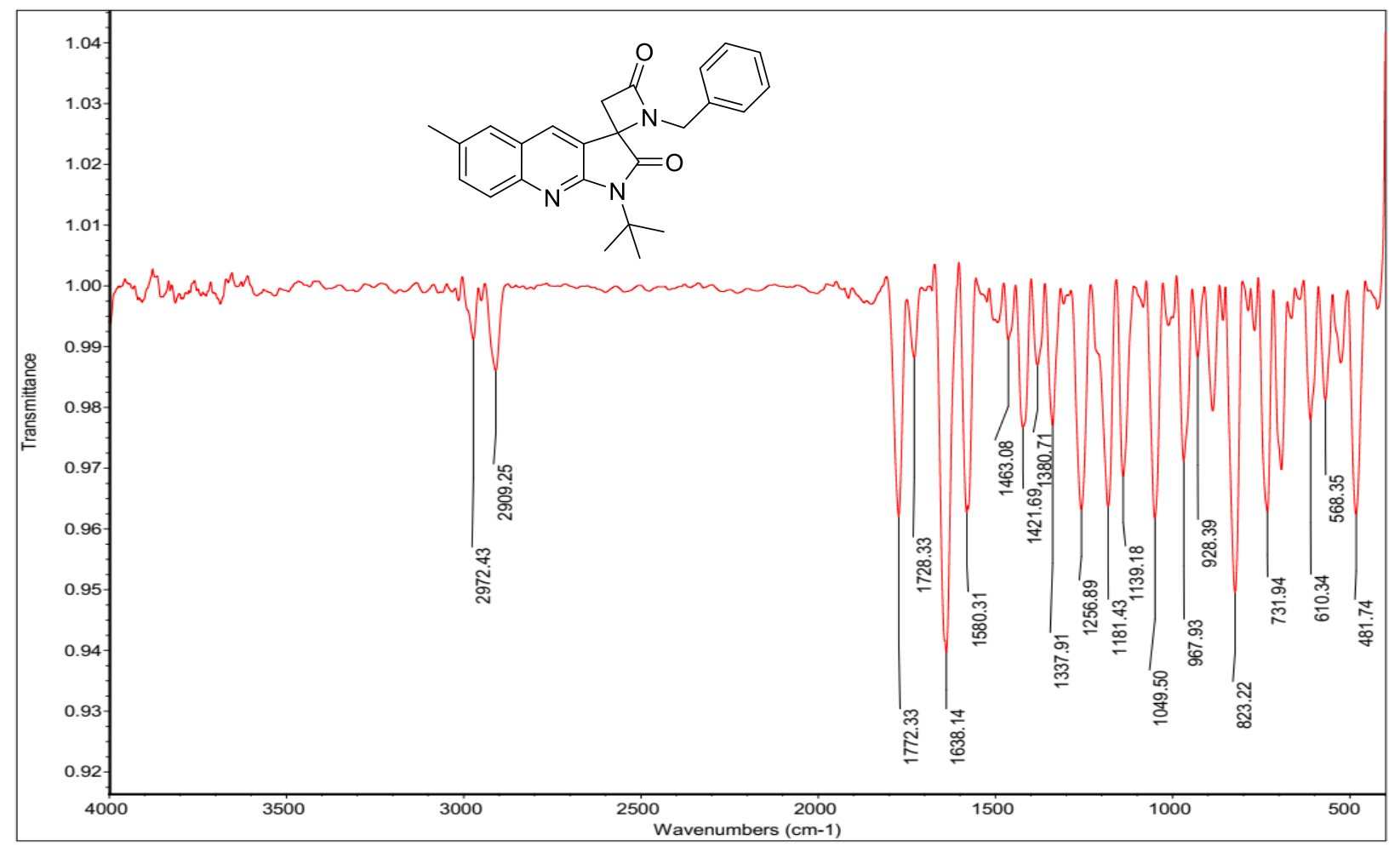

Figure S126: IR of compound $6 \mathrm{~h}\left(\mathrm{KBr}, \mathrm{cm}^{-1}\right)$ 


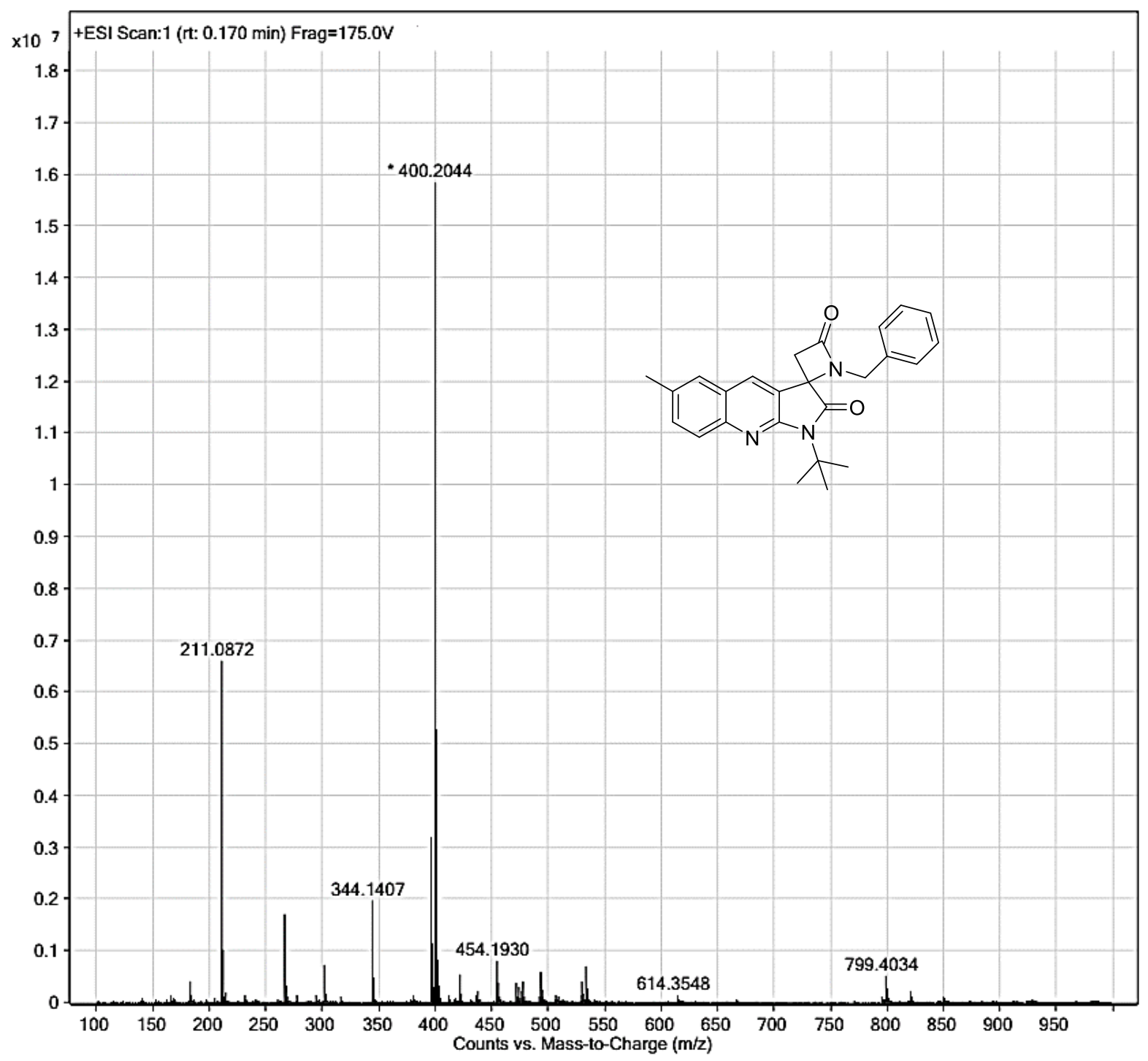

Figure S127: HRMS-ESI of $\mathbf{6 h}$ with formula $\mathrm{C}_{25} \mathrm{H}_{25} \mathrm{~N}_{3} \mathrm{O}_{2}$ and $[\mathrm{M}+\mathrm{H}]^{+} 400.2035$; and $[2 \mathrm{M}+\mathrm{H}]^{+}$ 799.4024. 


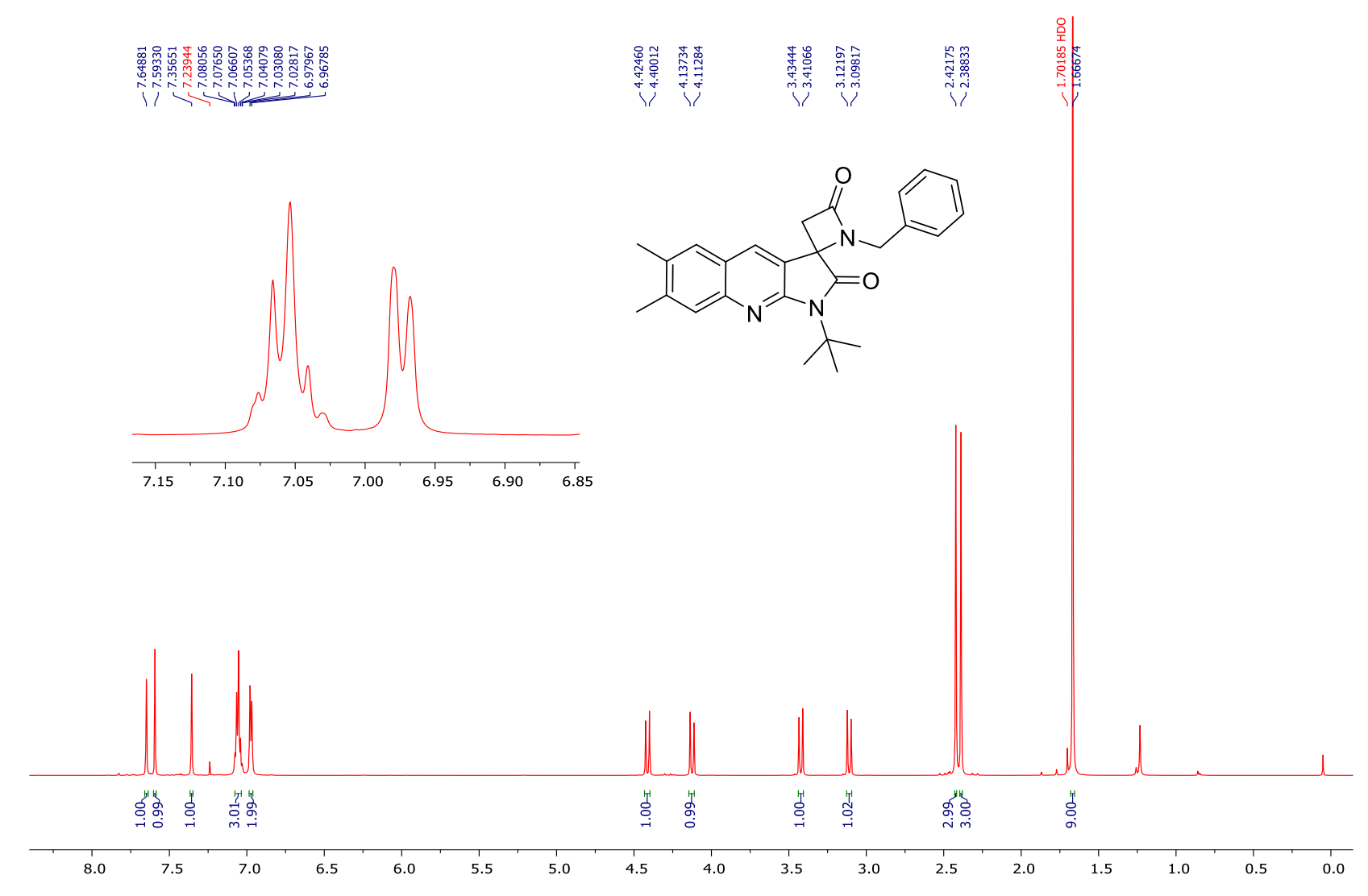

Figure S128: ${ }^{1} \mathrm{H}-\mathrm{NMR}$ of compound 6i $\left(600 \mathrm{MHz}, \mathrm{CDCl}_{3}\right)$

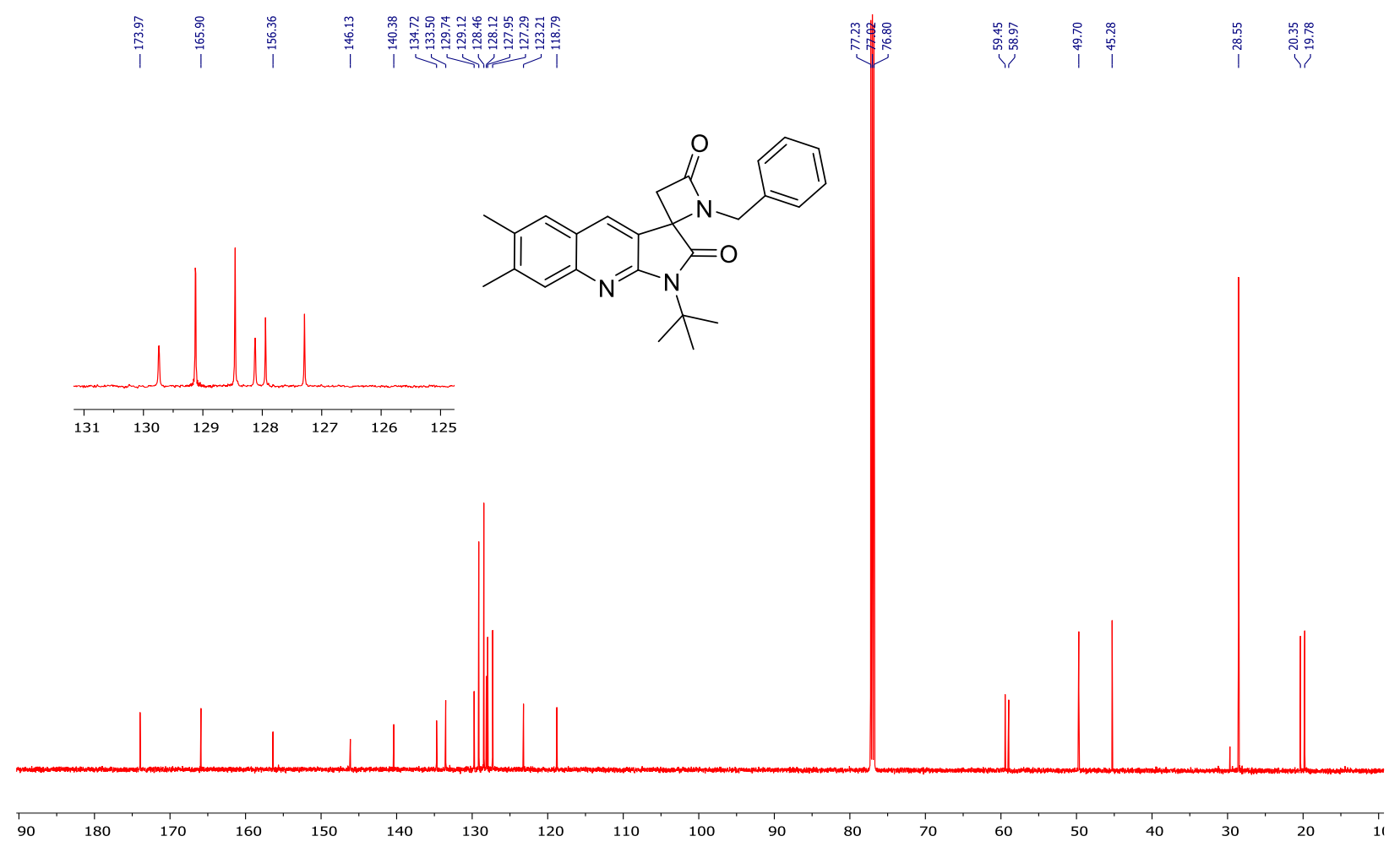

Figure S129: ${ }^{13} \mathrm{C}-\mathrm{NMR}$ of compound $\mathbf{6 i}\left(150 \mathrm{MHz}, \mathrm{CDCl}_{3}\right)$ 


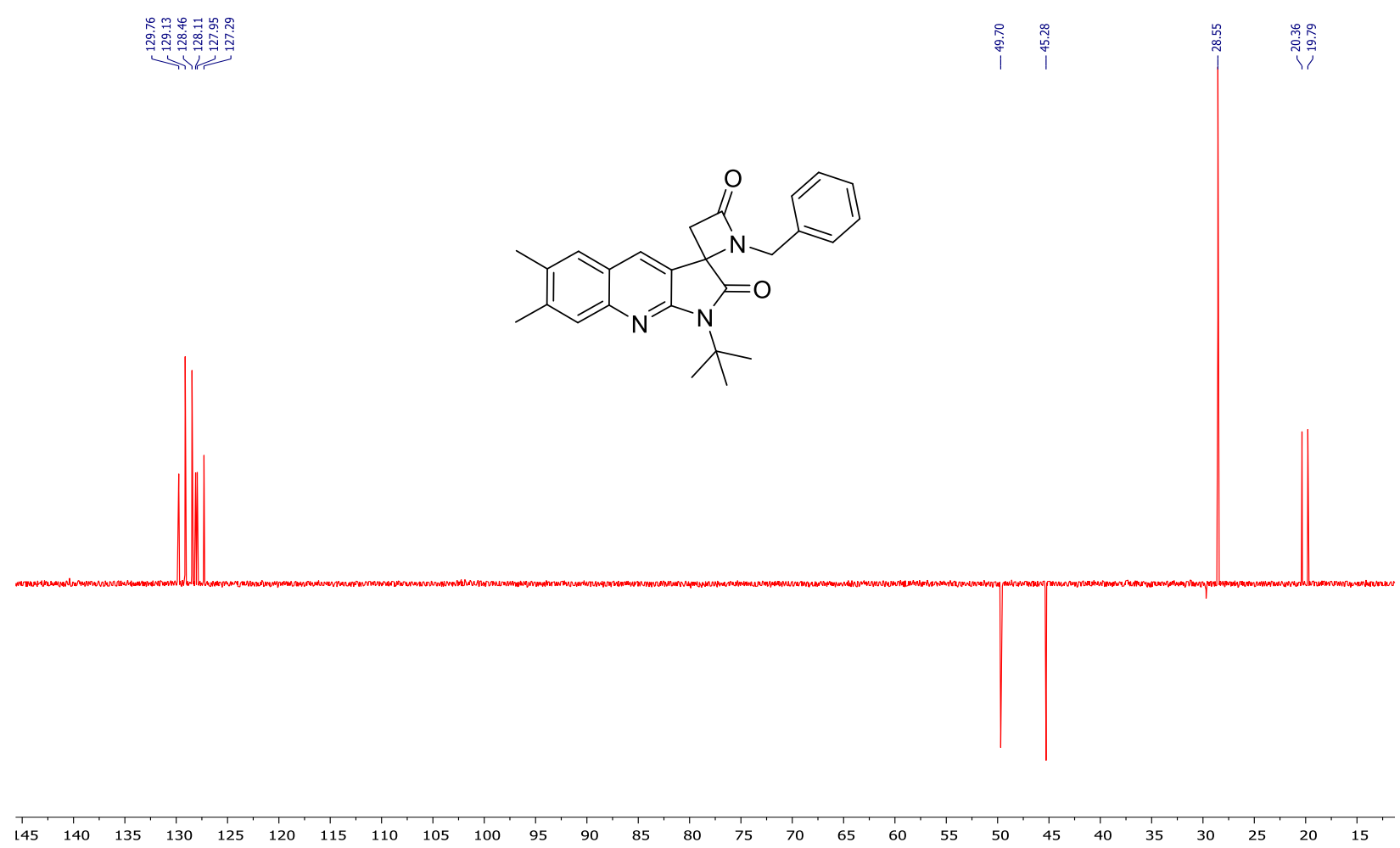

Figure S130: ${ }^{13} \mathrm{C}-\mathrm{NMR}$ (DEPT 135) of compound $6 \mathbf{i}\left(150 \mathrm{MHz}, \mathrm{CDCl}_{3}\right)$

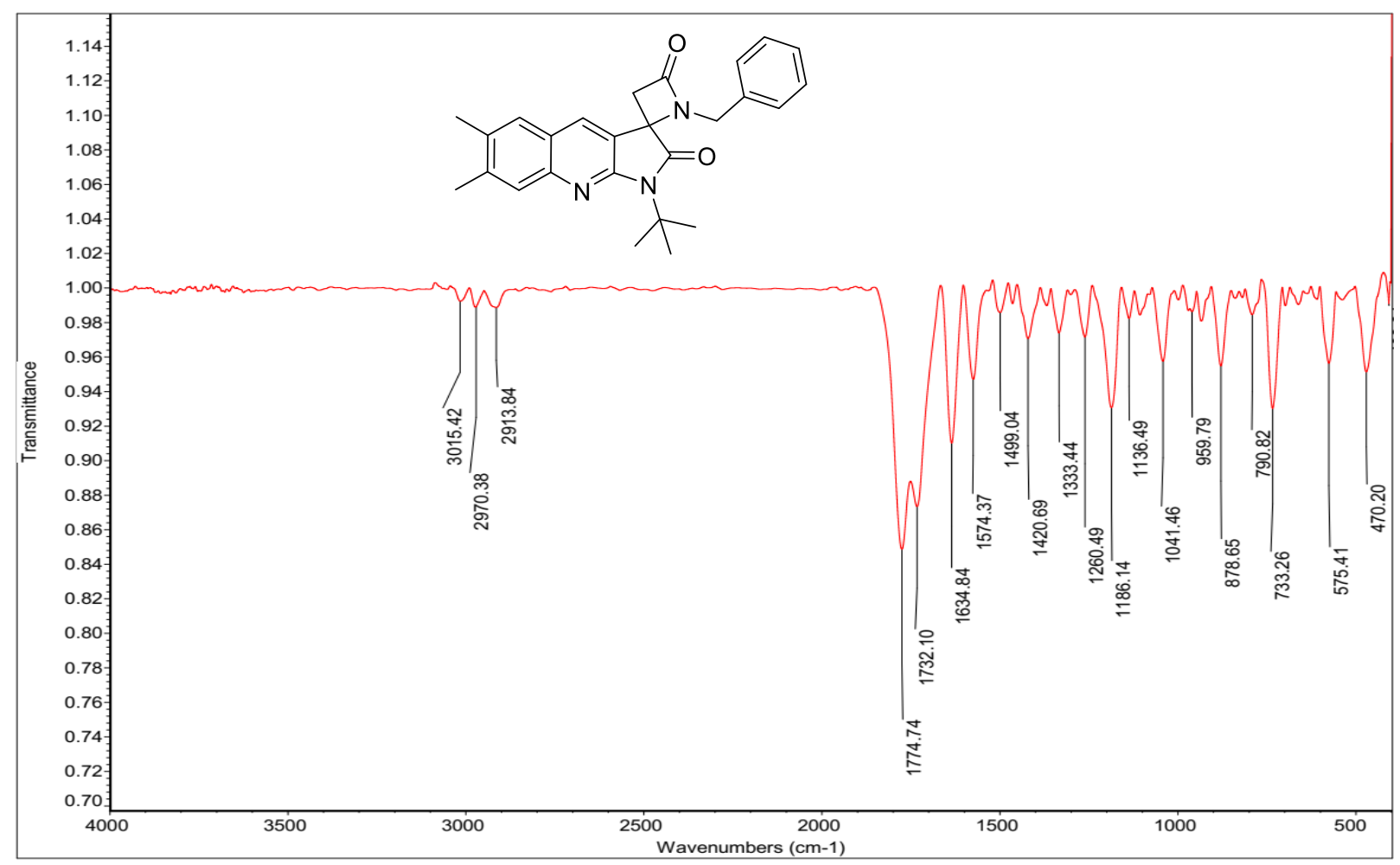

Figure S131: IR of compound $\mathbf{6 i}\left(\mathrm{KBr}, \mathrm{cm}^{-1}\right)$ 


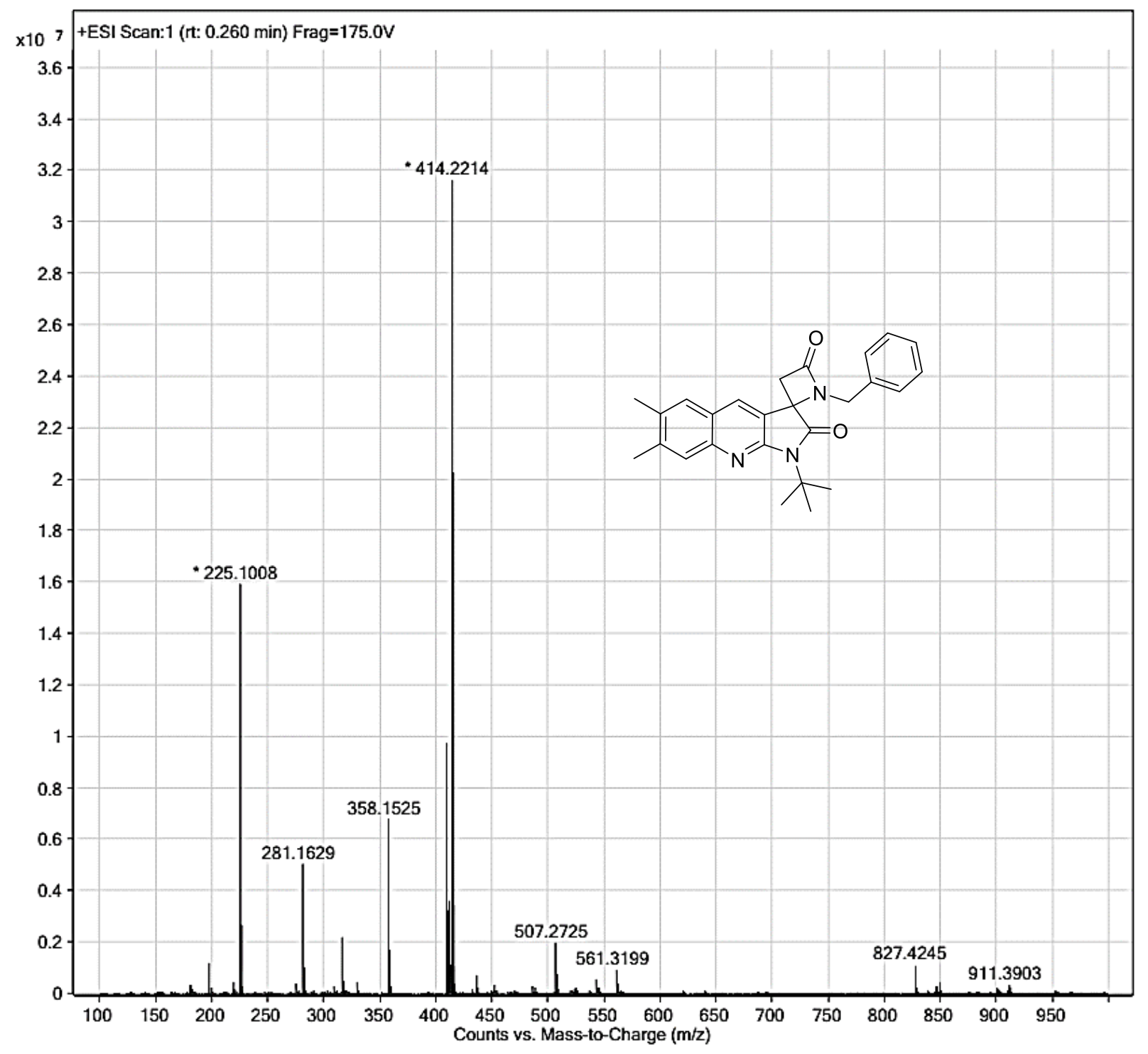

Figure S132: HRMS-ESI of $6 \mathbf{i}$ with formula $\mathrm{C}_{26} \mathrm{H}_{27} \mathrm{~N}_{3} \mathrm{O}_{2}$ and $[\mathrm{M}+\mathrm{H}]^{+}$414.2205; and [2M+H] ${ }^{+}$ 827.4236 . 

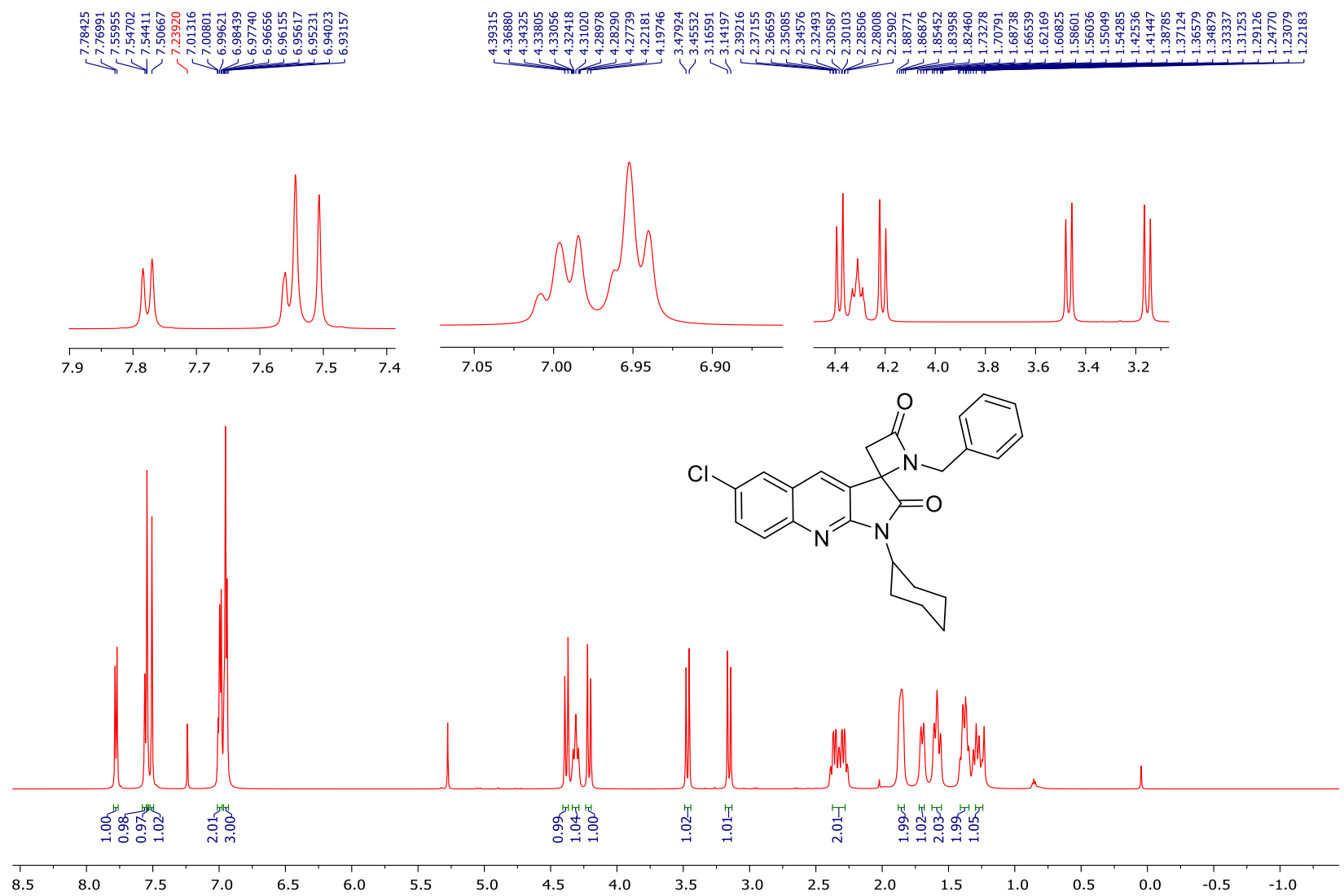

Figure S133: ${ }^{1} \mathrm{H}-\mathrm{NMR}$ of compound $\mathbf{6 j}\left(600 \mathrm{MHz}, \mathrm{CDCl}_{3}\right)$

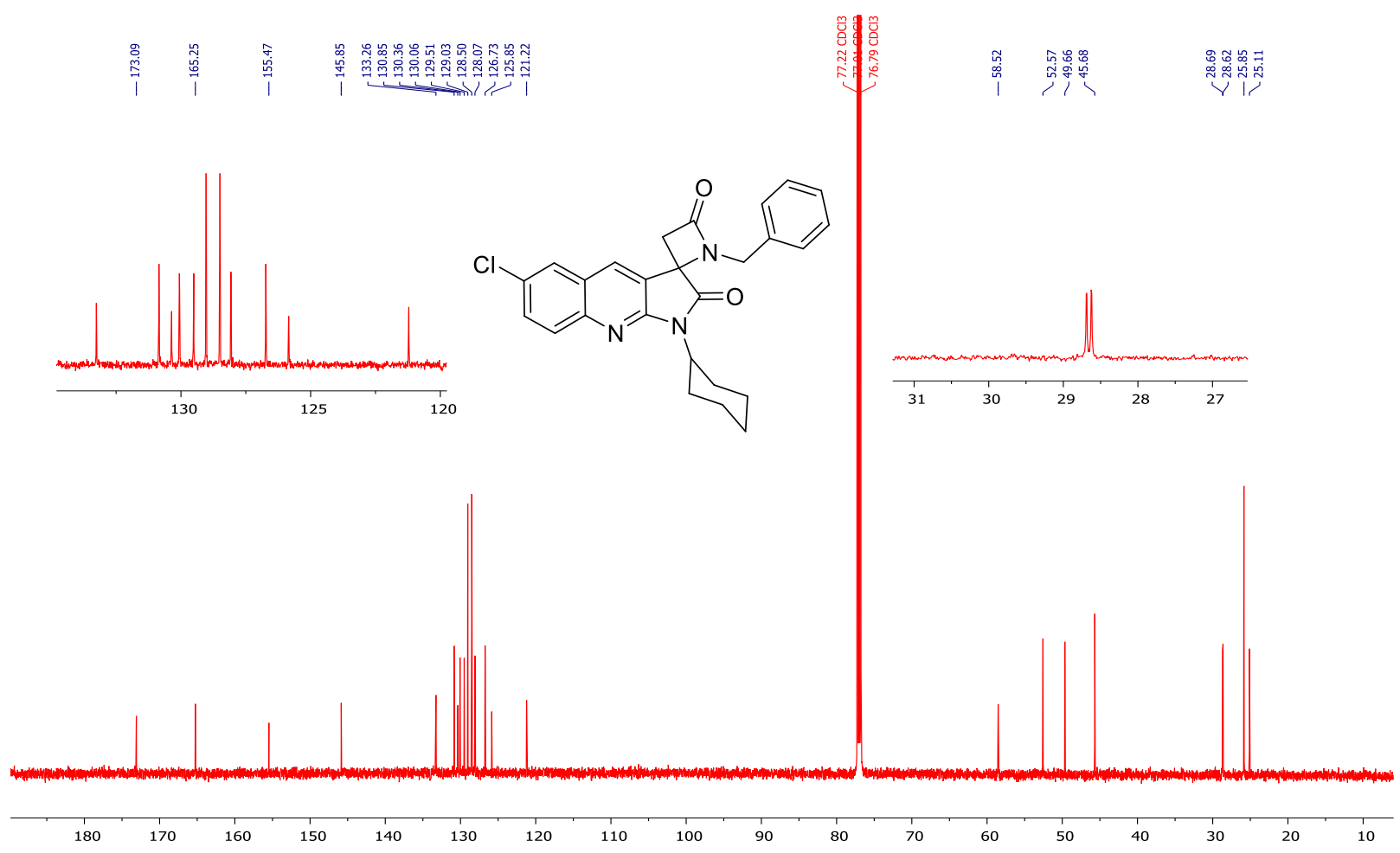

Figure S134: ${ }^{13} \mathrm{C}-\mathrm{NMR}$ of compound $\mathbf{6 j}\left(150 \mathrm{MHz}, \mathrm{CDCl}_{3}\right)$ 

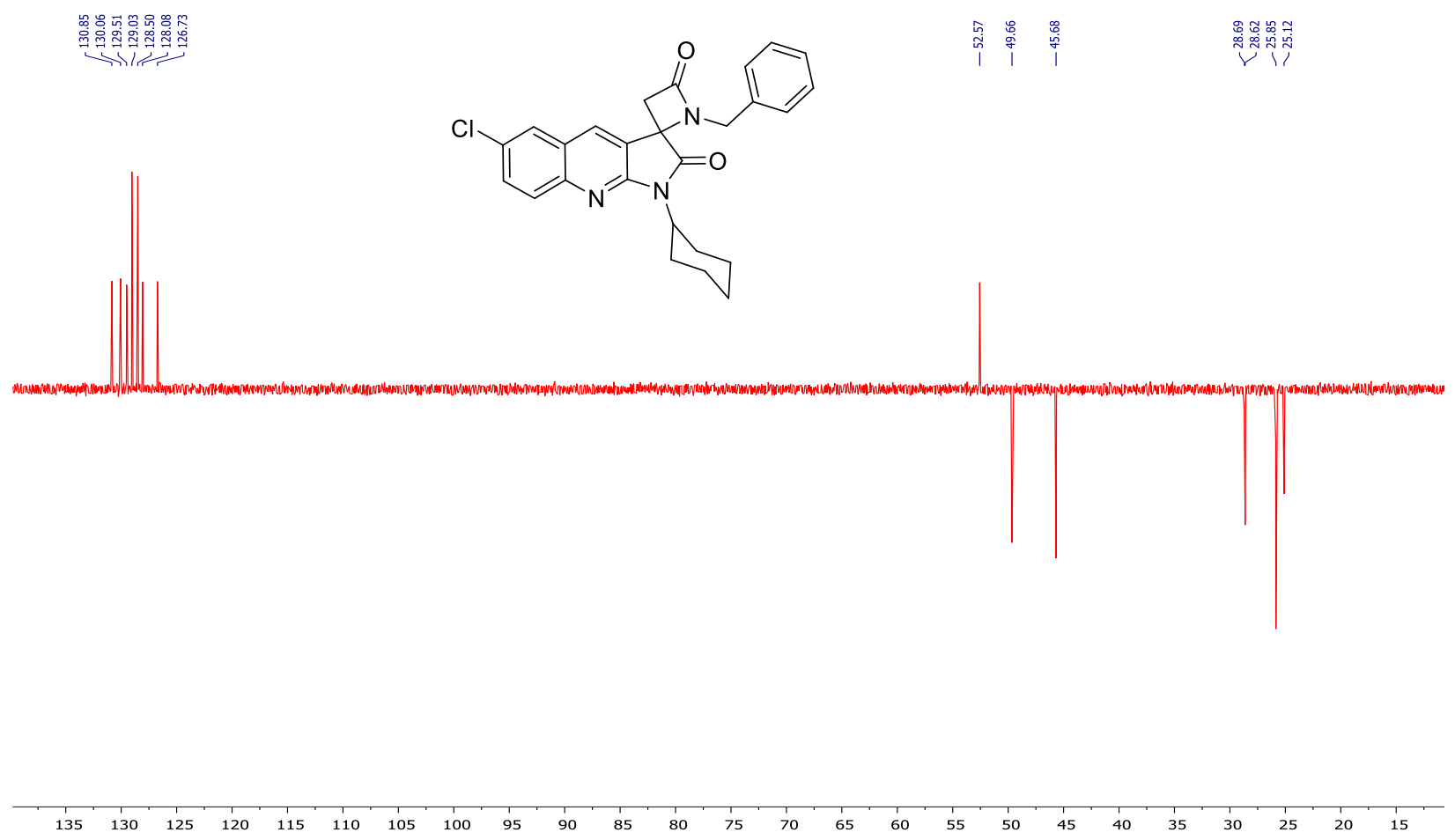

Figure S135: ${ }^{13} \mathrm{C}-\mathrm{NMR}$ (DEPT 135) of compound $\mathbf{6 j}\left(150 \mathrm{MHz}, \mathrm{CDCl}_{3}\right)$

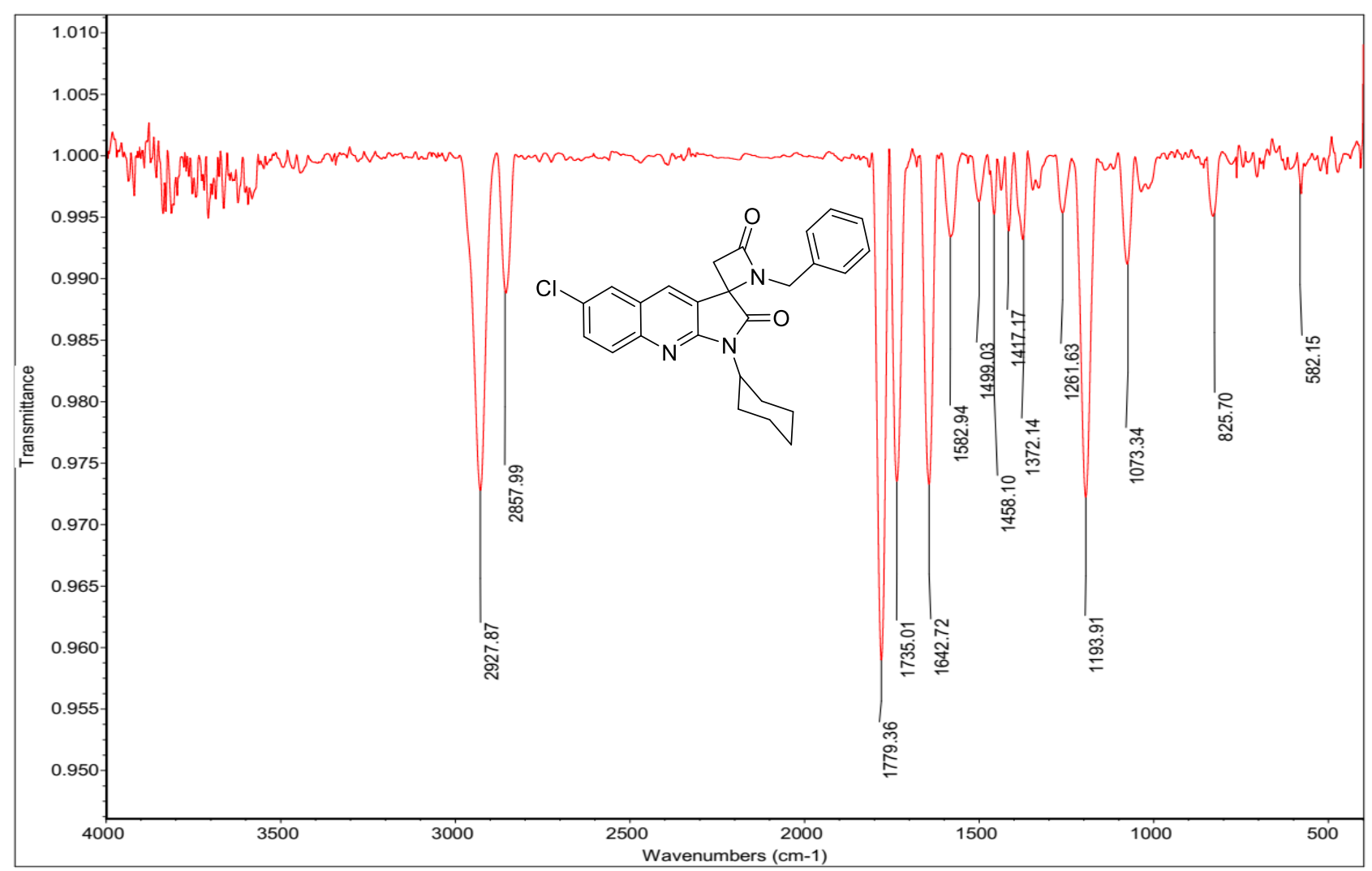

Figure S136: IR of compound $\mathbf{6 j}\left(\mathrm{KBr}, \mathrm{cm}^{-1}\right)$ 


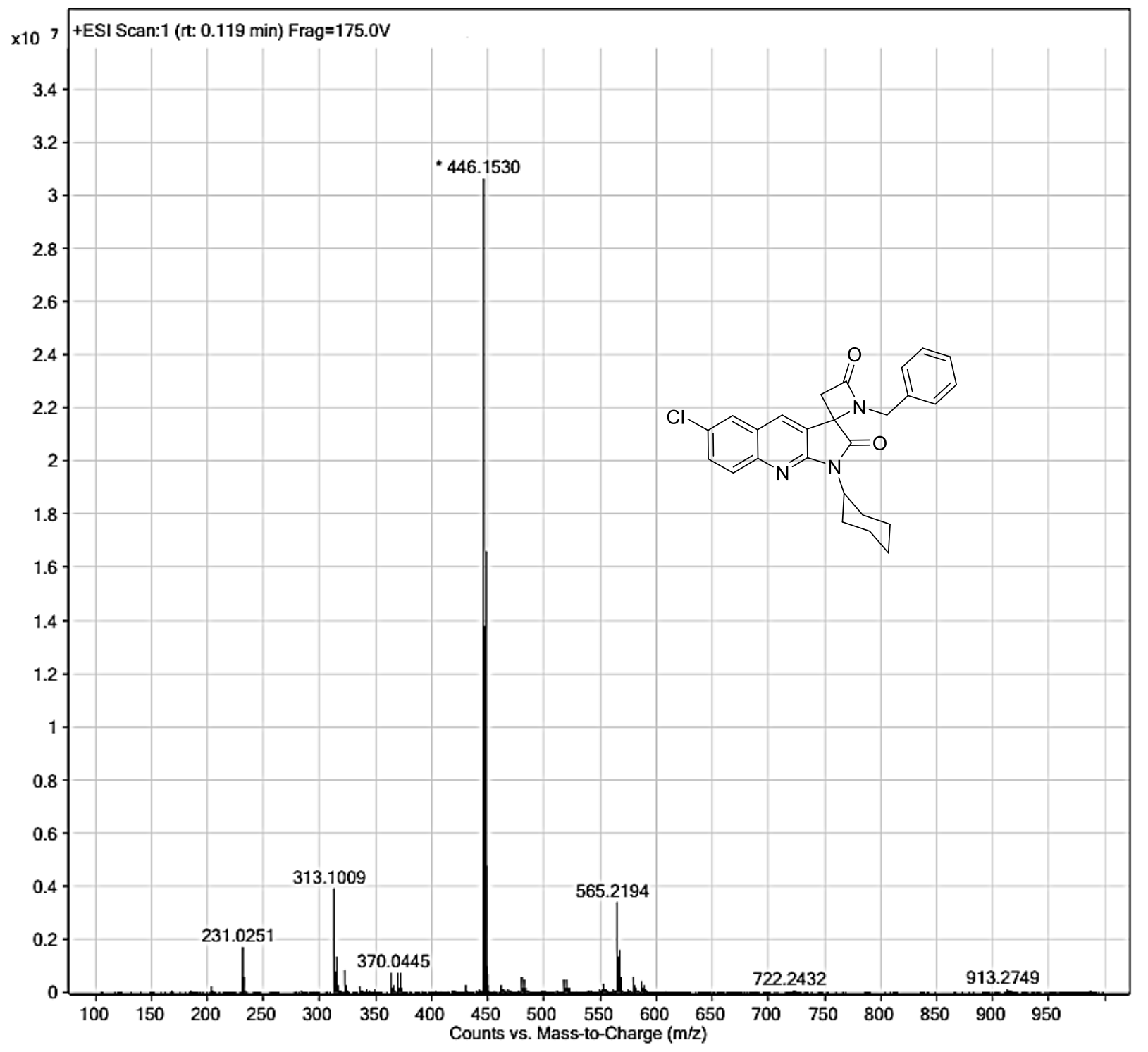

Figure S137: HRMS-ESI of $\mathbf{6 j}$ with formula $\mathrm{C}_{26} \mathrm{H}_{24} \mathrm{ClN}_{3} \mathrm{O}_{2}$ and $[\mathrm{M}+\mathrm{H}]^{+} 446.1521$; and $[2 \mathrm{M}+\mathrm{Na}]^{+}$913.2740. 

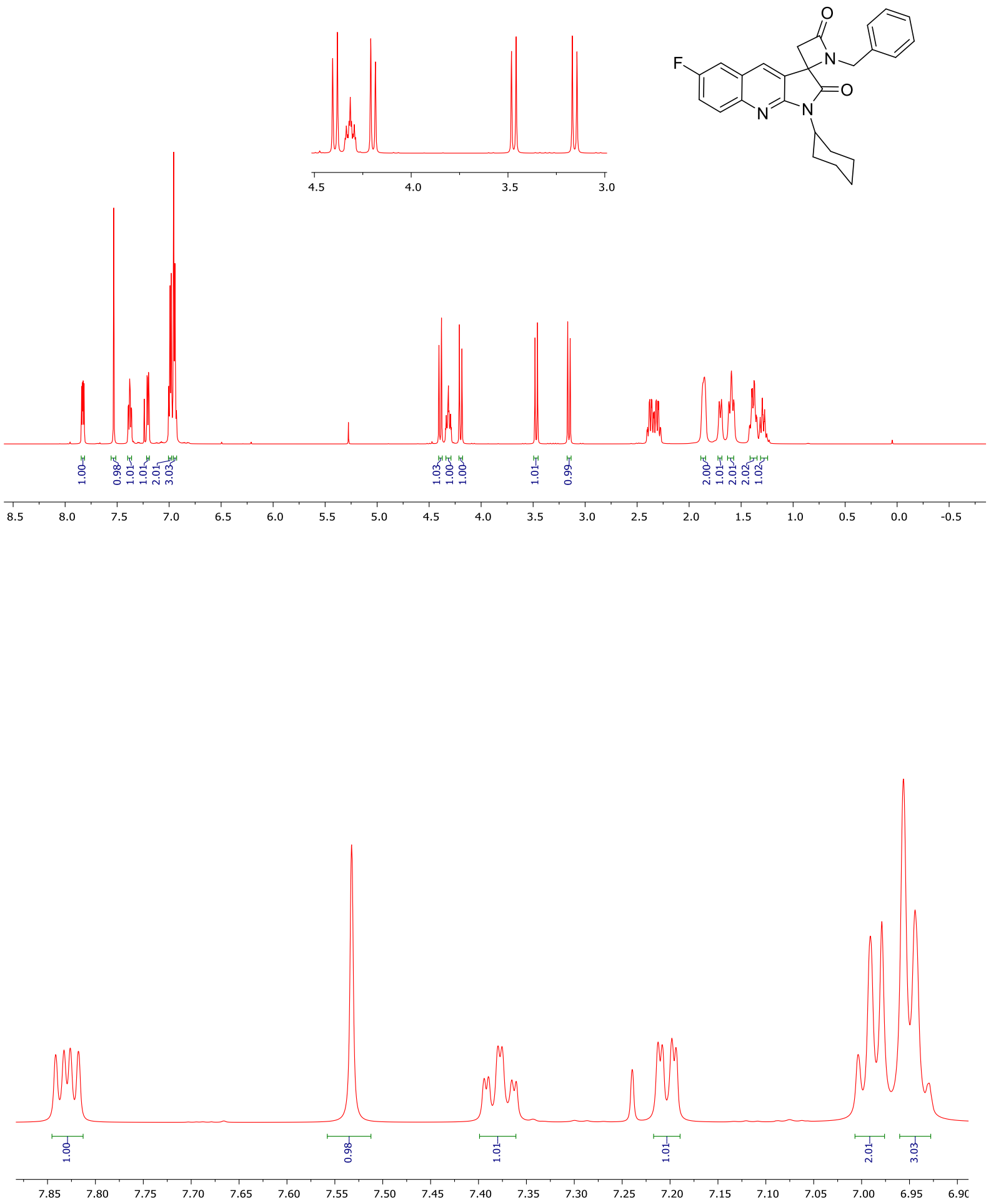

Figure S138: ${ }^{1} \mathrm{H}-\mathrm{NMR}$ of compound $\mathbf{6 k}\left(600 \mathrm{MHz}, \mathrm{CDCl}_{3}\right)$ 

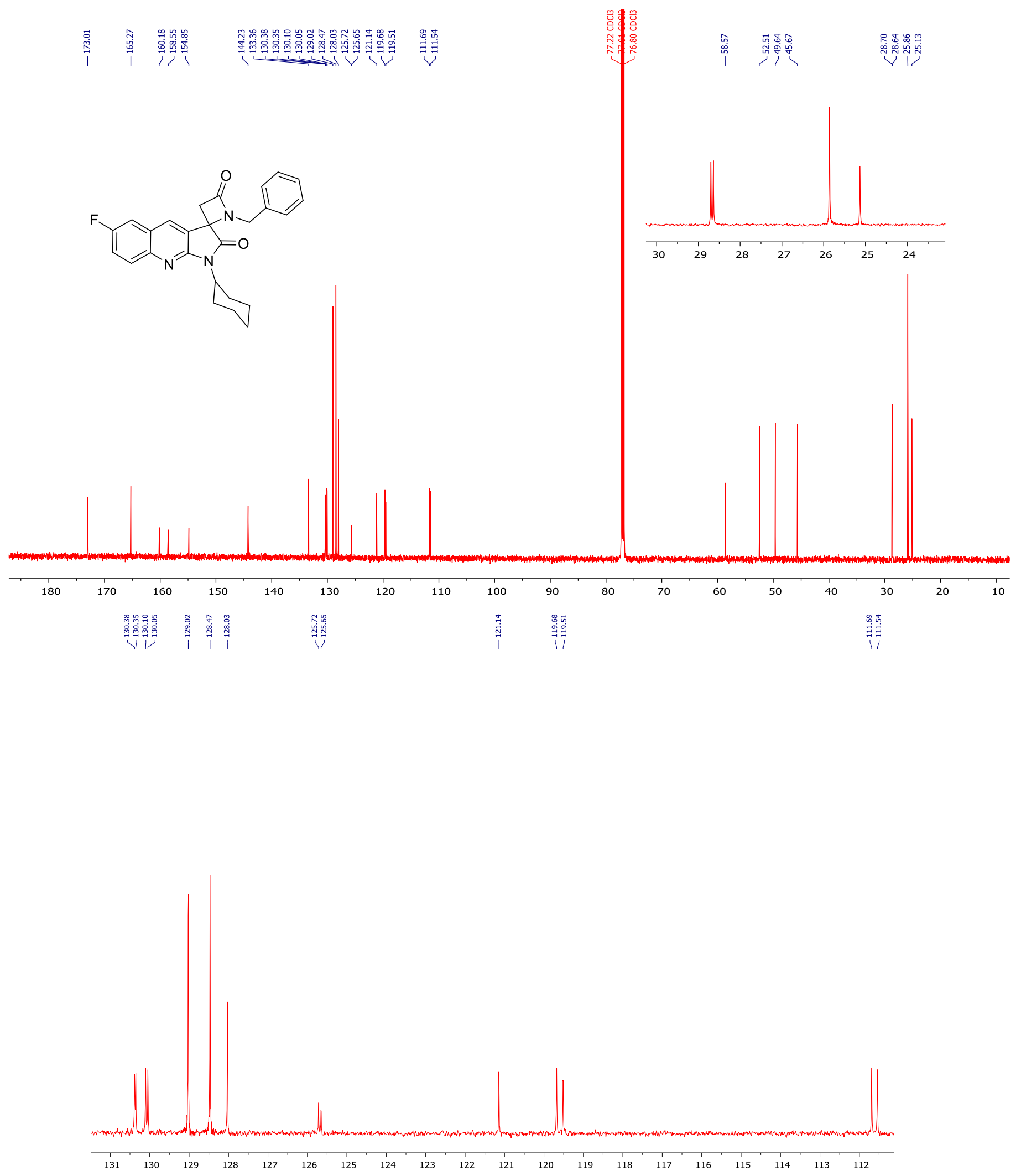

Figure S139: ${ }^{13} \mathrm{C}-\mathrm{NMR}$ of compound $\mathbf{6 k}\left(150 \mathrm{MHz}, \mathrm{CDCl}_{3}\right)$ 


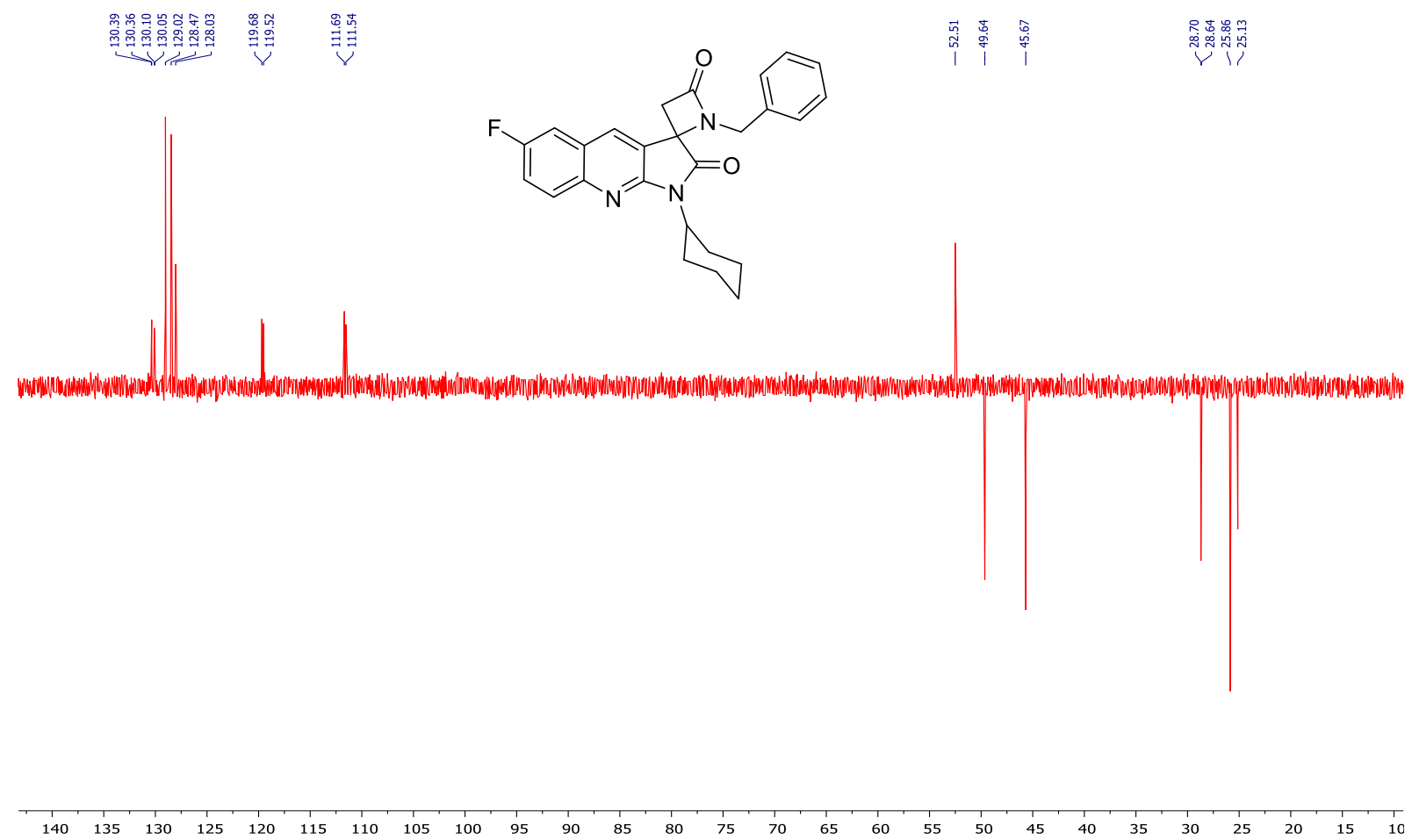

Figure S140: ${ }^{13} \mathrm{C}-\mathrm{NMR}$ (DEPT 135) of compound 6k (150 MHz, $\mathrm{CDCl}_{3}$ )

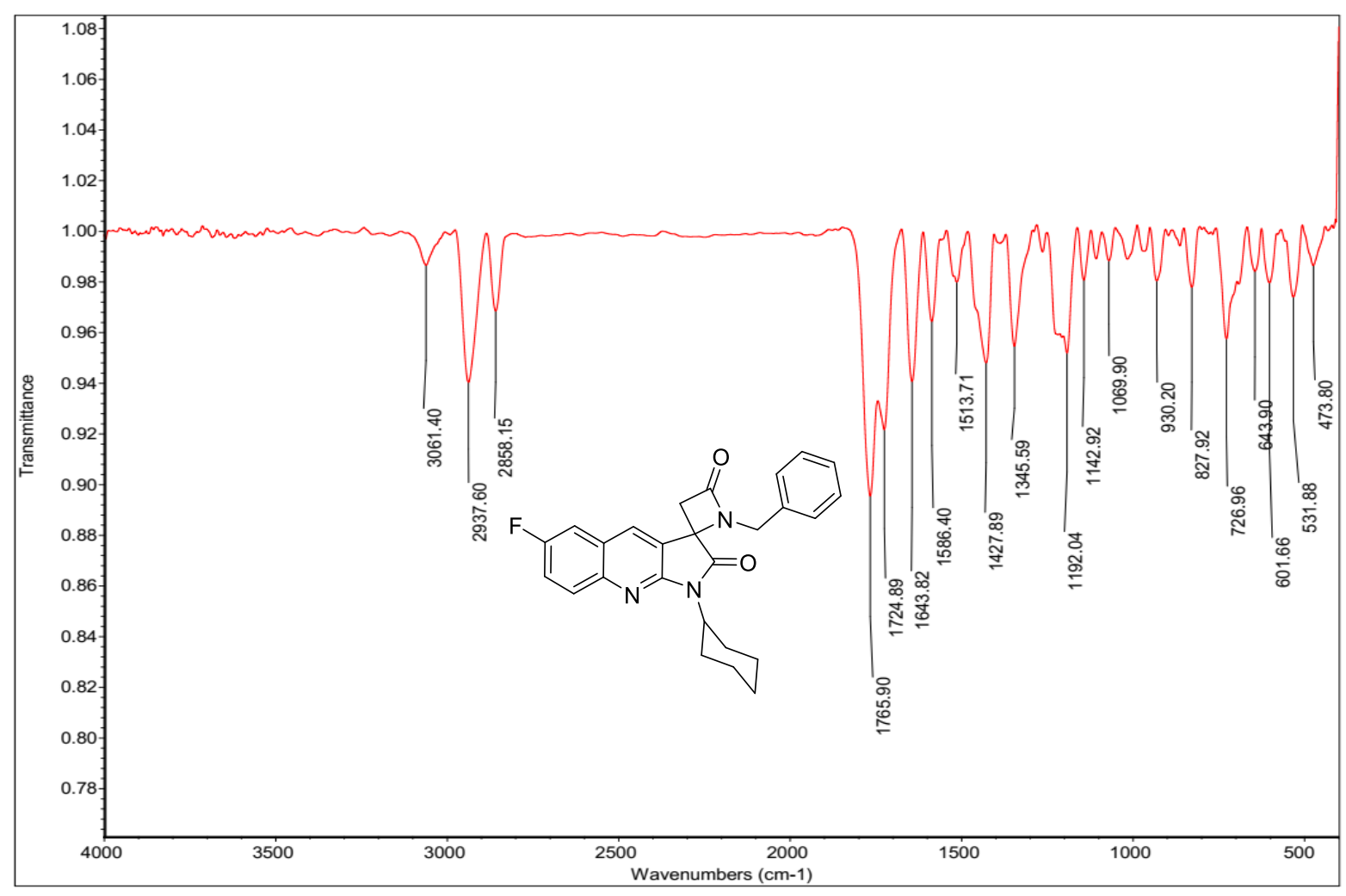

Figure S141: IR of compound 6k $\left(\mathrm{KBr}, \mathrm{cm}^{-1}\right)$ 


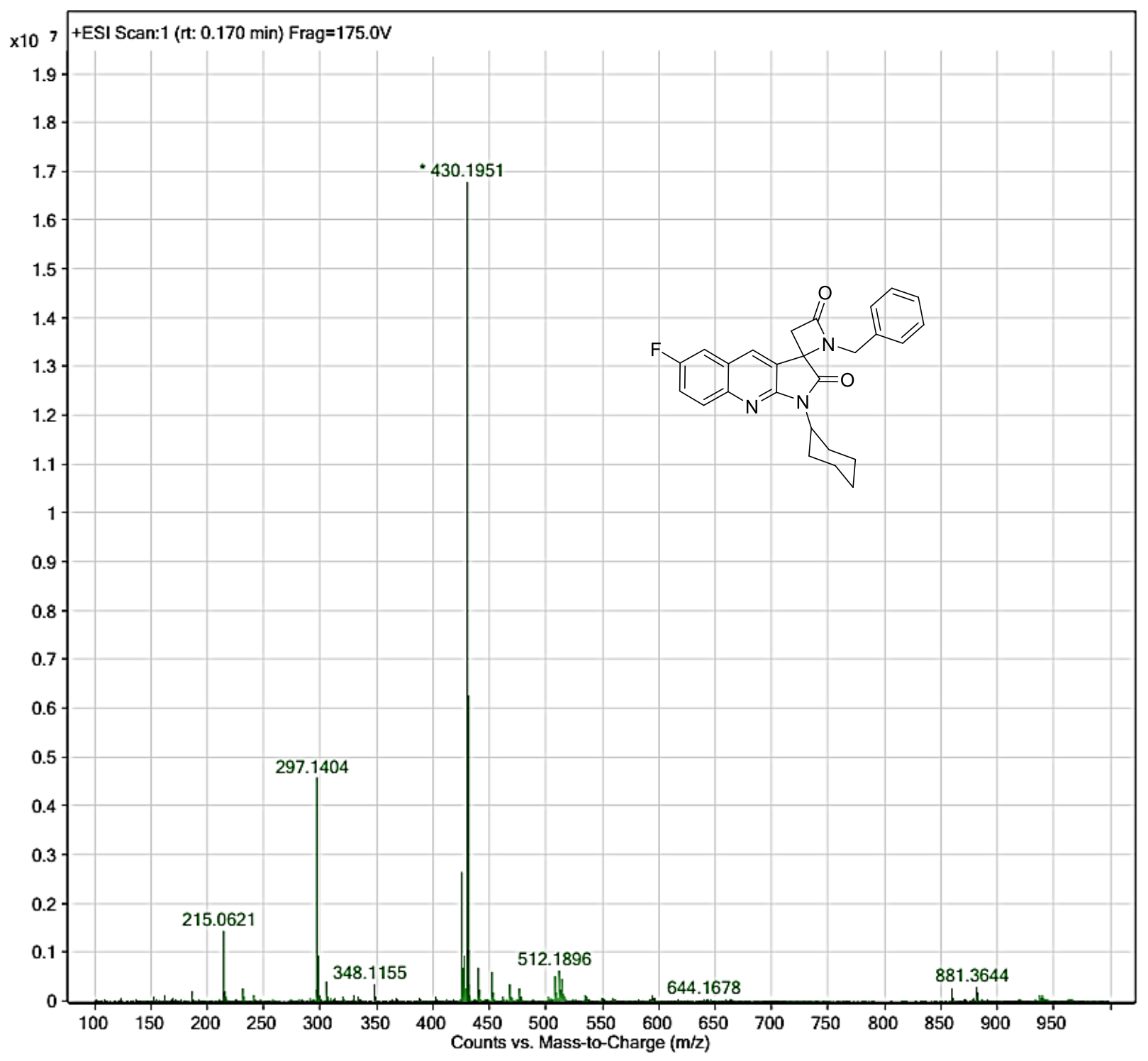

Figure S142: HRMS-ESI of $6 \mathbf{k}$ with formula $\mathrm{C}_{26} \mathrm{H}_{24} \mathrm{FN}_{3} \mathrm{O}_{2}$ and $[\mathrm{M}+\mathrm{H}]^{+} 430.1941$; and $[2 \mathrm{M}+\mathrm{Na}]^{+} 881.3635$; and $[\mathrm{M}+2 \mathrm{H}]^{2+} 215.0613$. 


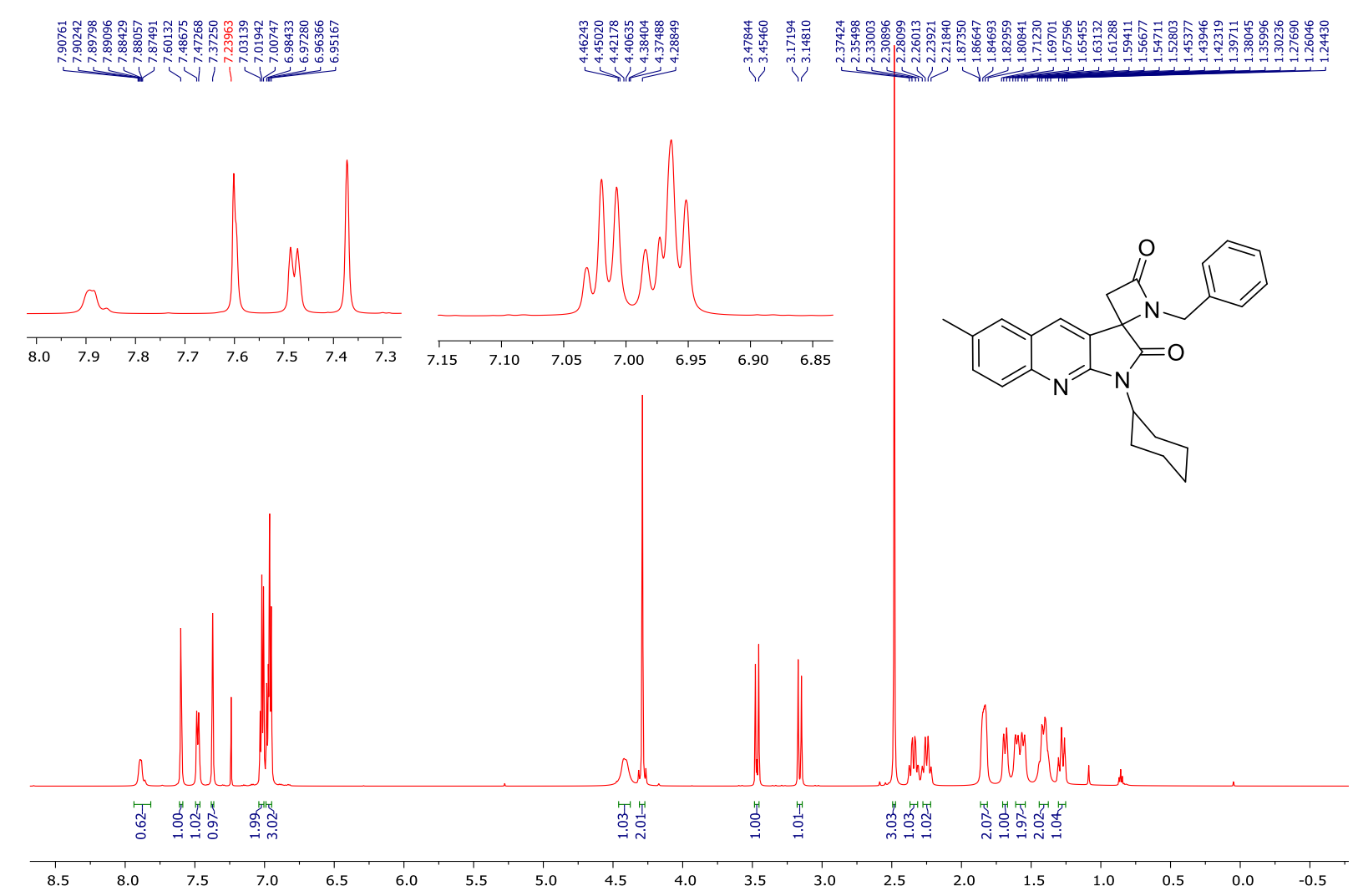

Figure S143: ${ }^{1} \mathrm{H}-\mathrm{NMR}$ of compound $61\left(600 \mathrm{MHz}, \mathrm{CDCl}_{3}\right)$

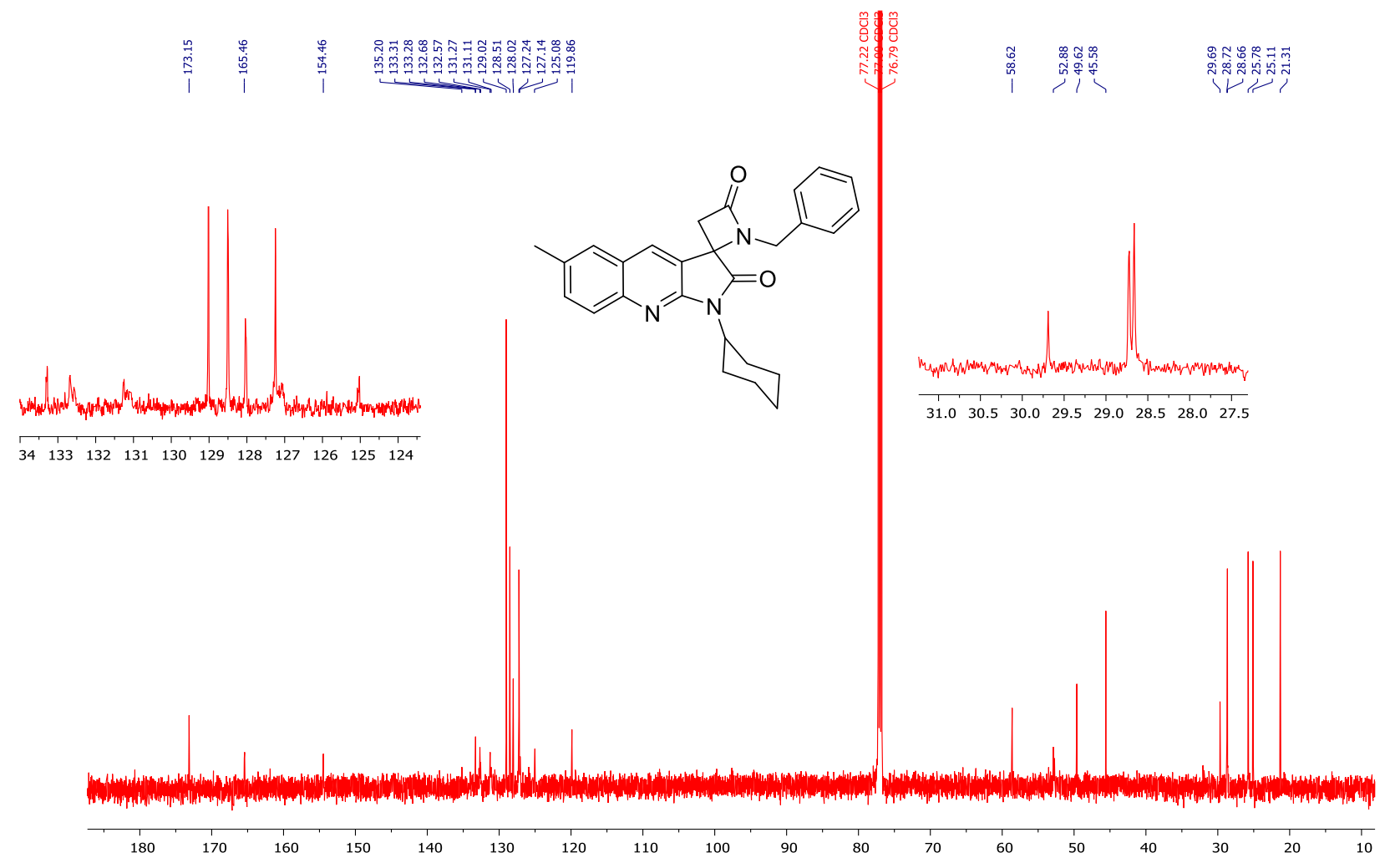

Figure S144: ${ }^{13} \mathrm{C}$-NMR of compound $61\left(150 \mathrm{MHz}, \mathrm{CDCl}_{3}\right)$ 


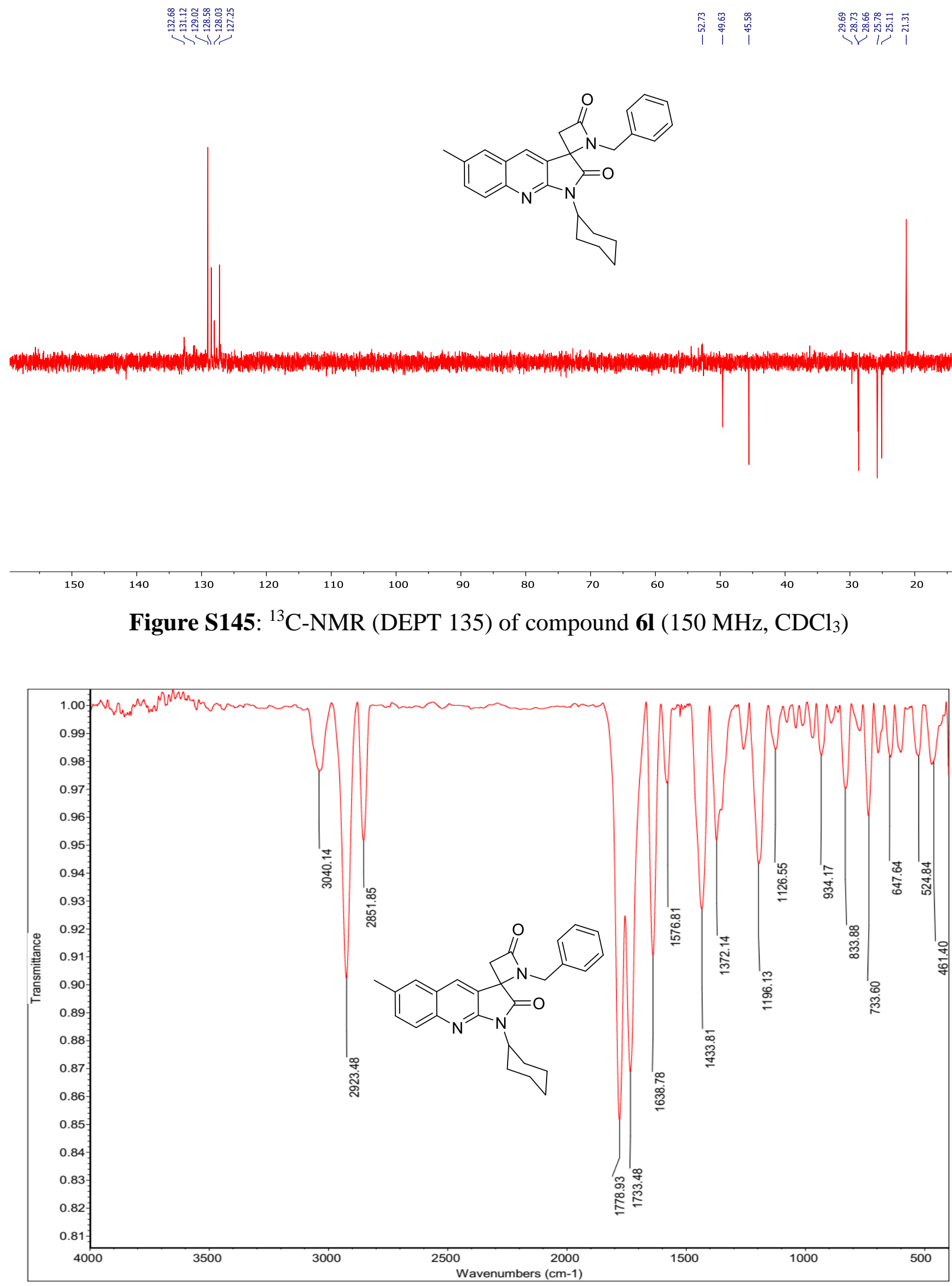

Figure S146: IR of compound $61\left(\mathrm{KBr}, \mathrm{cm}^{-1}\right)$ 


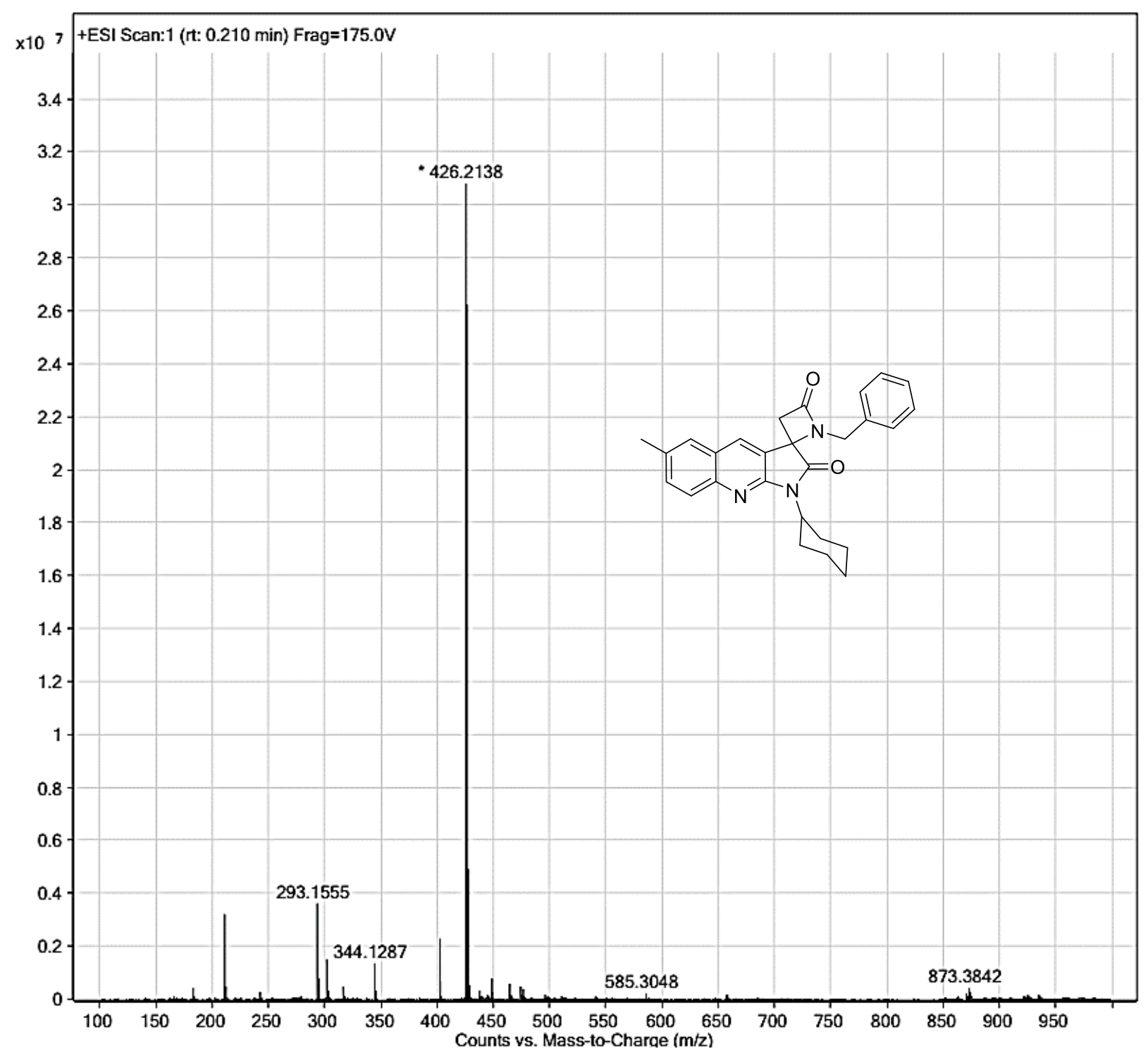

Figure S147: HRMS-ESI of 61 with formula $\mathrm{C}_{27} \mathrm{H}_{27} \mathrm{~N}_{3} \mathrm{O}_{2}$ and $[\mathrm{M}+\mathrm{H}]^{+} 426.2130$; and [2M+Na] ${ }^{+}$ 873.3833. 


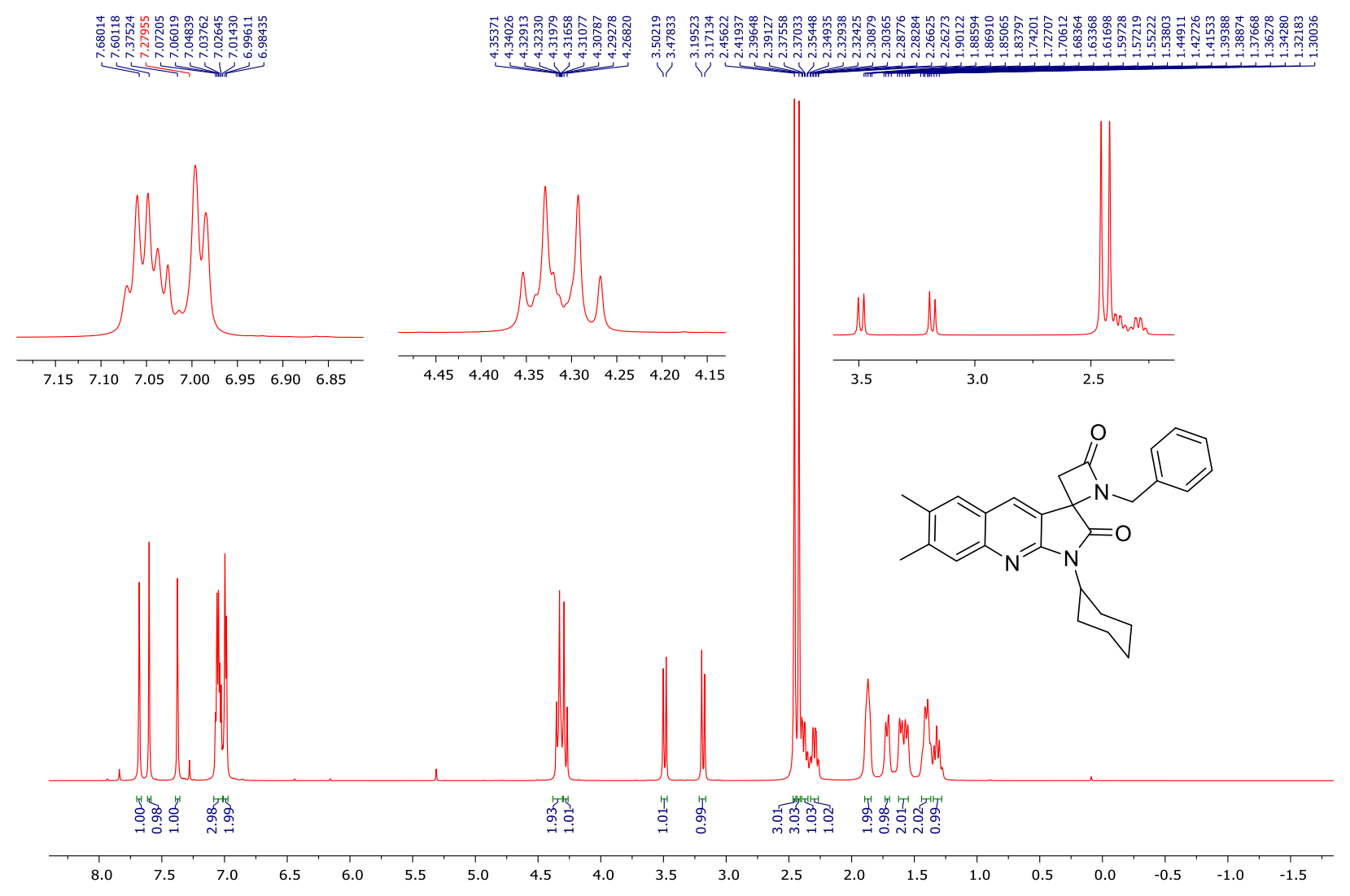

Figure S148: ${ }^{1} \mathrm{H}-\mathrm{NMR}$ of compound $\mathbf{6 m}\left(600 \mathrm{MHz}, \mathrm{CDCl}_{3}\right)$

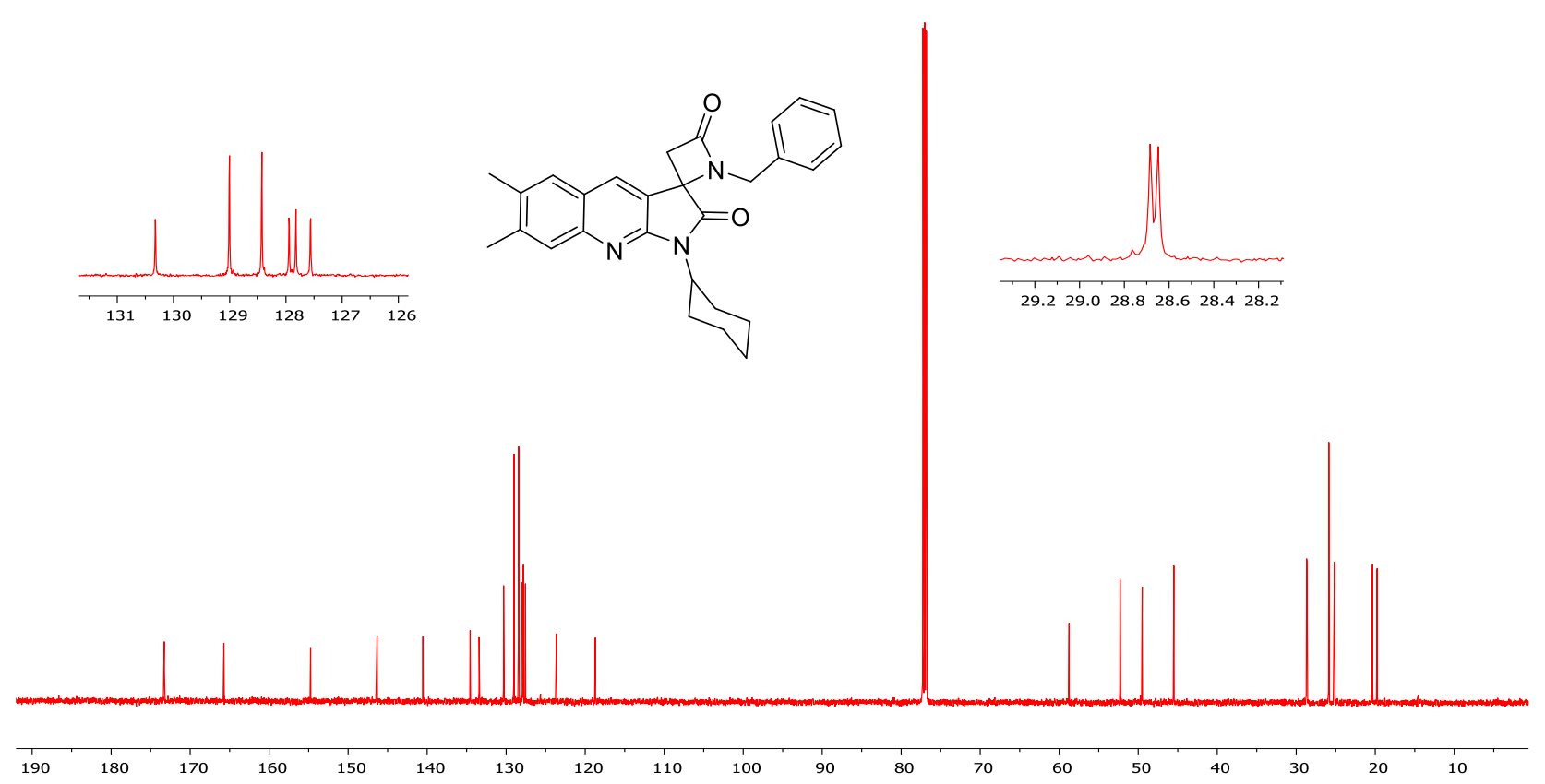

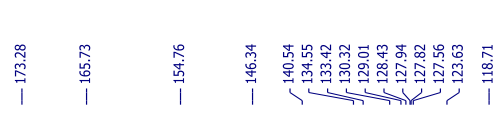

Figure S149: ${ }^{13} \mathrm{C}-\mathrm{NMR}$ of compound $\mathbf{6 m}\left(150 \mathrm{MHz}, \mathrm{CDCl}_{3}\right)$ 

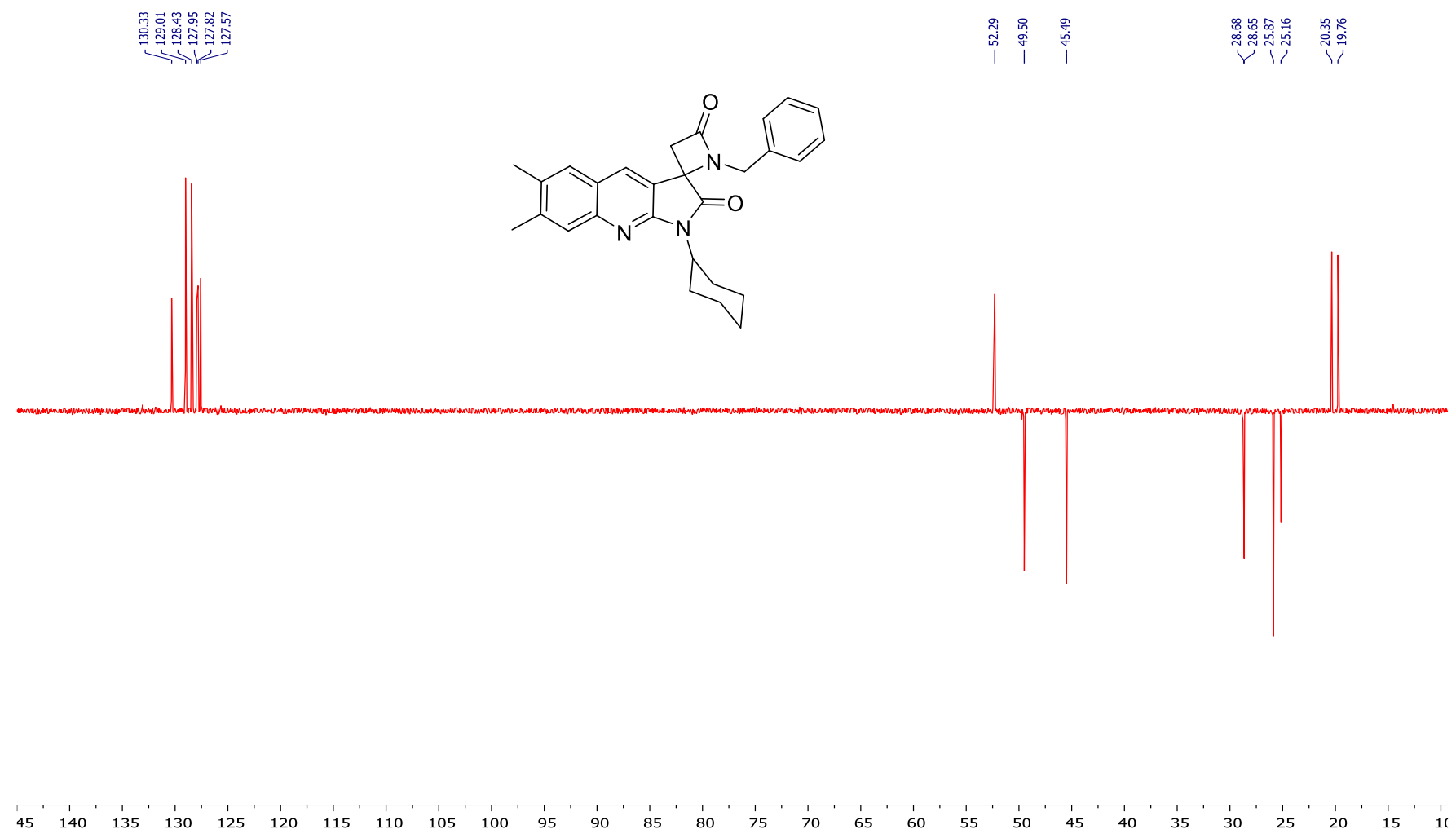

Figure S150: ${ }^{13} \mathrm{C}-\mathrm{NMR}$ (DEPT 135) of compound $6 \mathbf{m}\left(150 \mathrm{MHz}, \mathrm{CDCl}_{3}\right)$

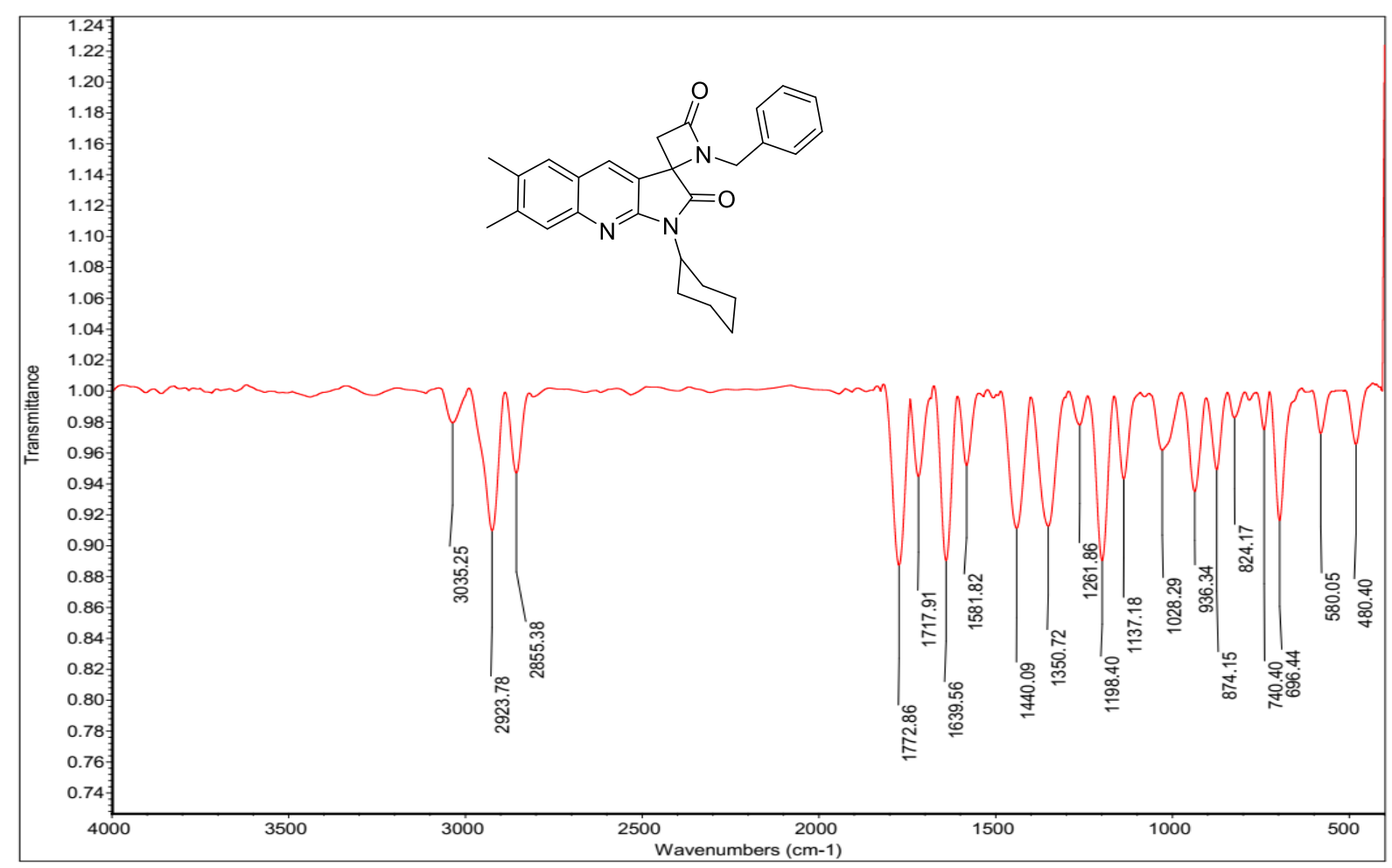

Figure S151: IR of compound $\mathbf{6 m}\left(\mathrm{KBr}, \mathrm{cm}^{-1}\right)$ 


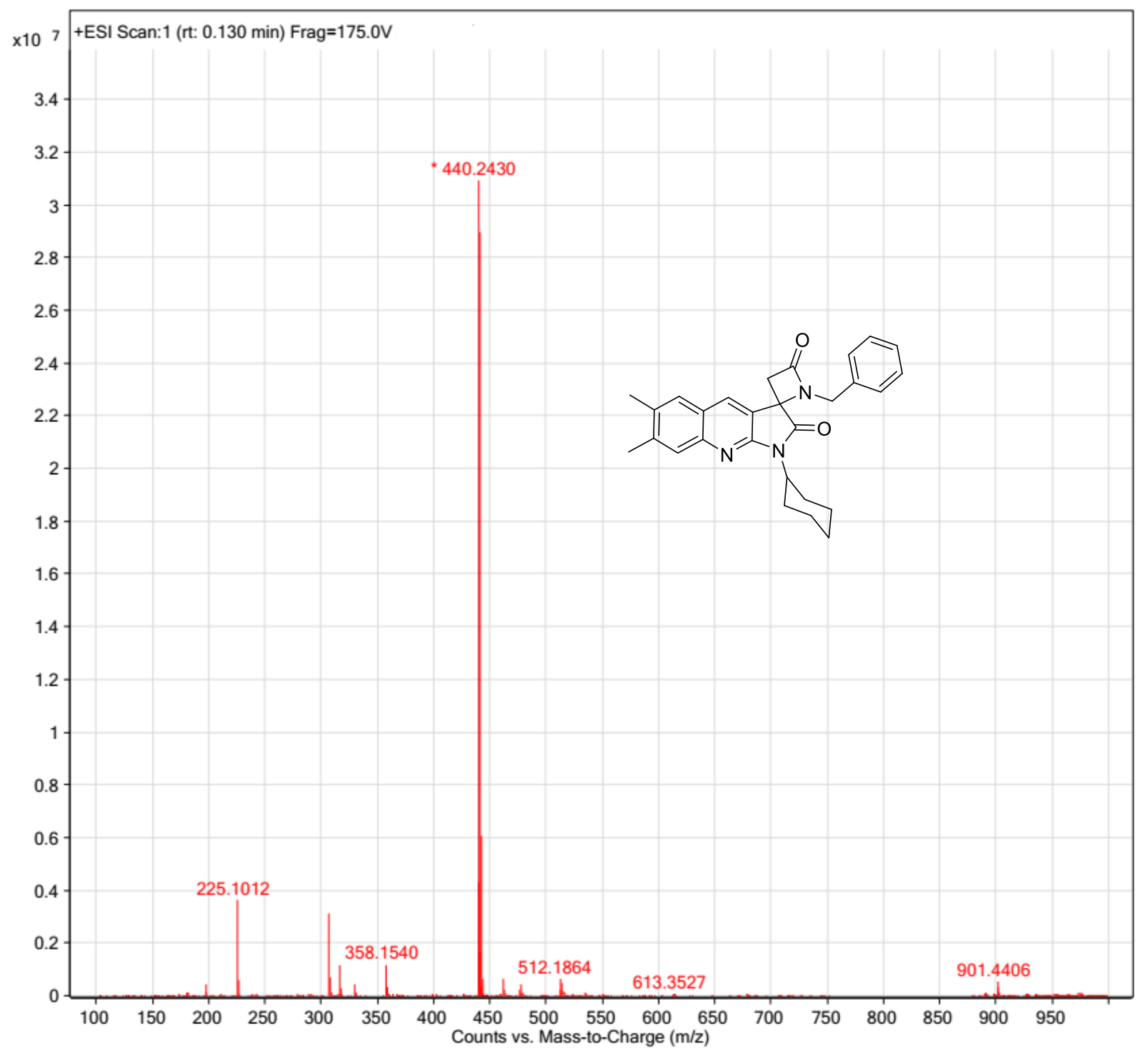

Figure S152: HRMS-ESI of $\mathbf{6 m}$ with formula $\mathrm{C}_{28} \mathrm{H}_{29} \mathrm{~N}_{3} \mathrm{O}_{2}$ and $[\mathrm{M}+\mathrm{H}]^{+} 440.2438$; and $[2 \mathrm{M}+\mathrm{Na}]^{+} 901.4415$. 


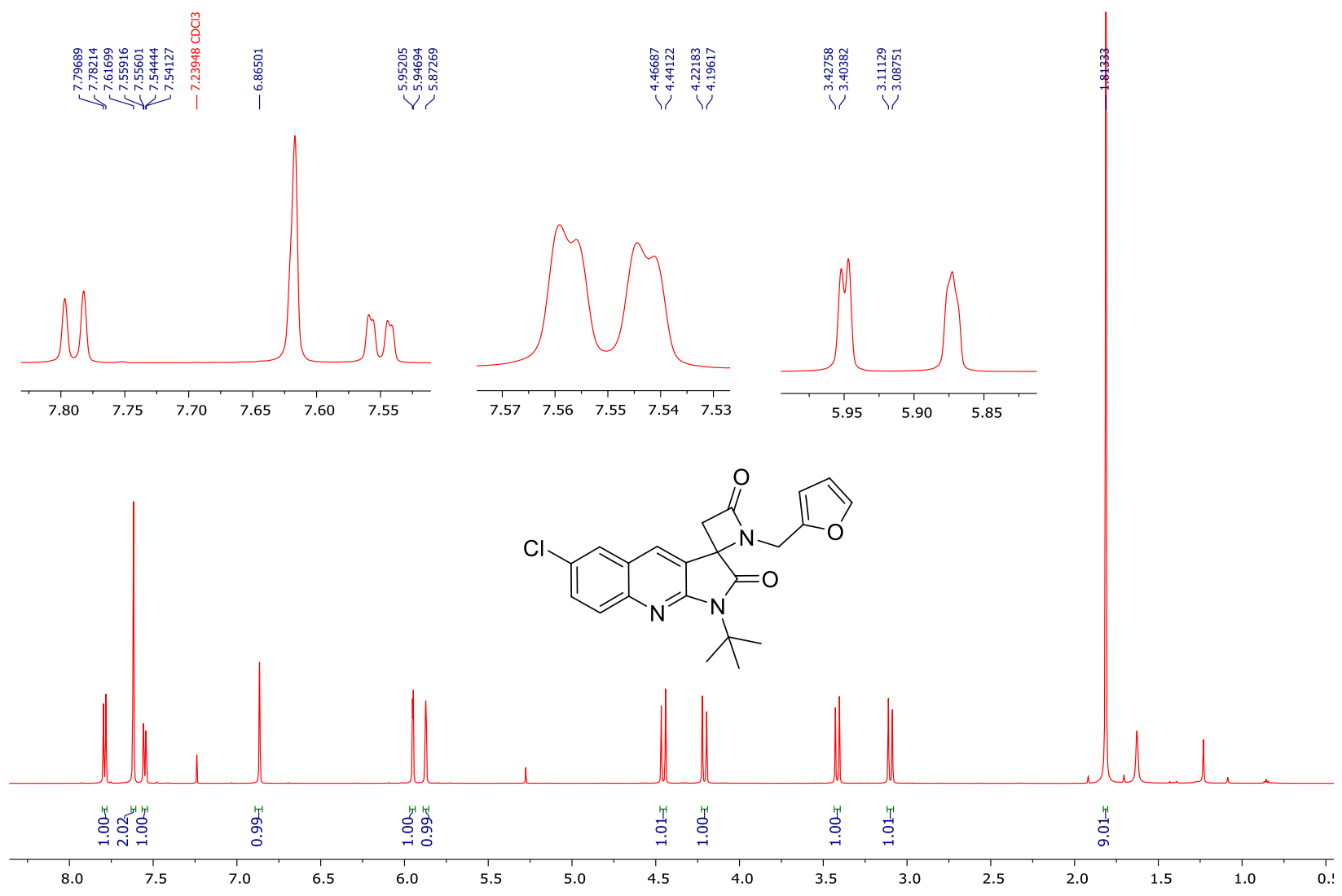

Figure S153: ${ }^{1} \mathrm{H}-\mathrm{NMR}$ of compound $\mathbf{6 n}\left(600 \mathrm{MHz}, \mathrm{CDCl}_{3}\right)$

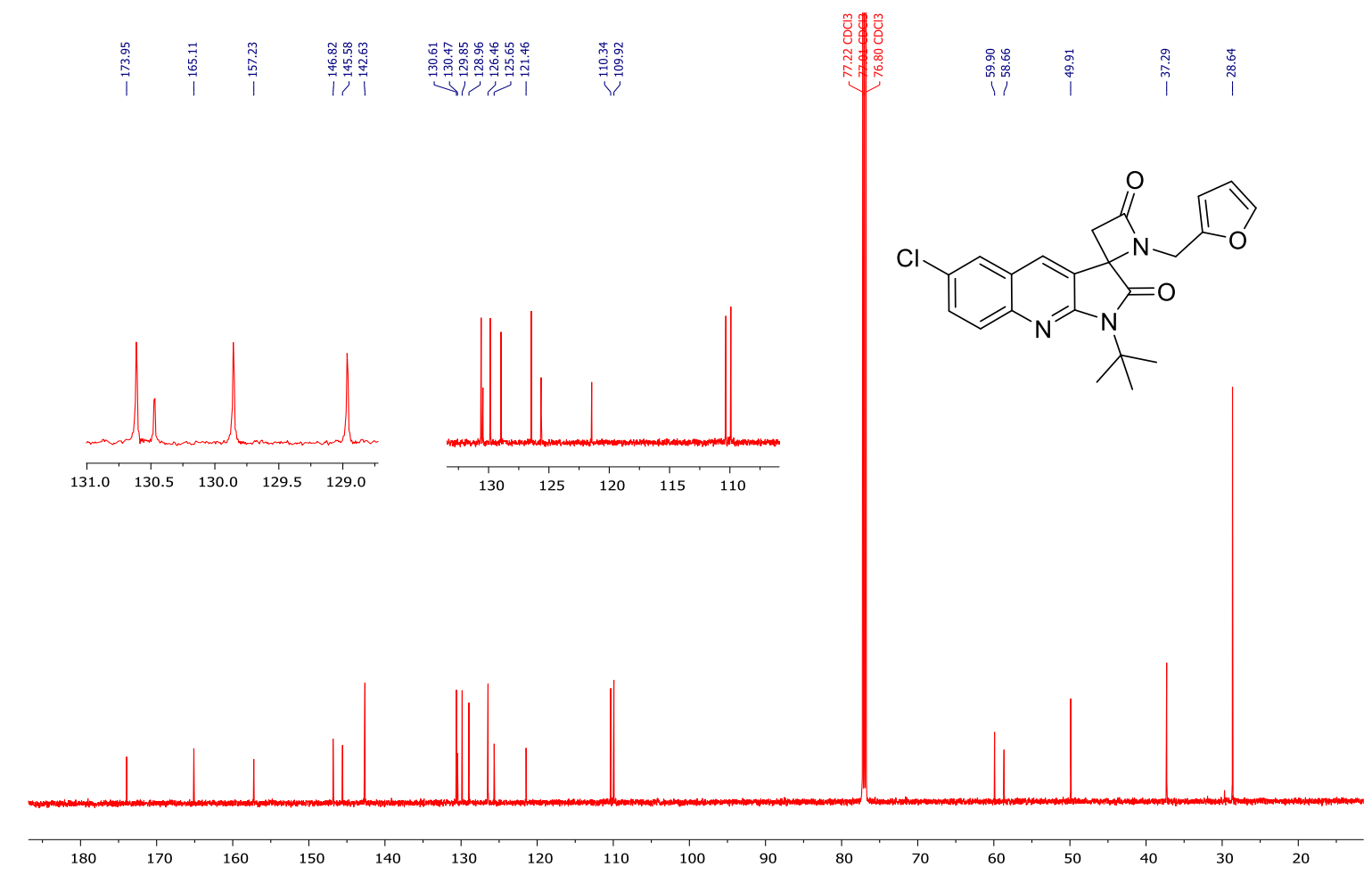

Figure S154: ${ }^{13} \mathrm{C}-\mathrm{NMR}$ of compound $\mathbf{6 n}\left(150 \mathrm{MHz}, \mathrm{CDCl}_{3}\right)$ 

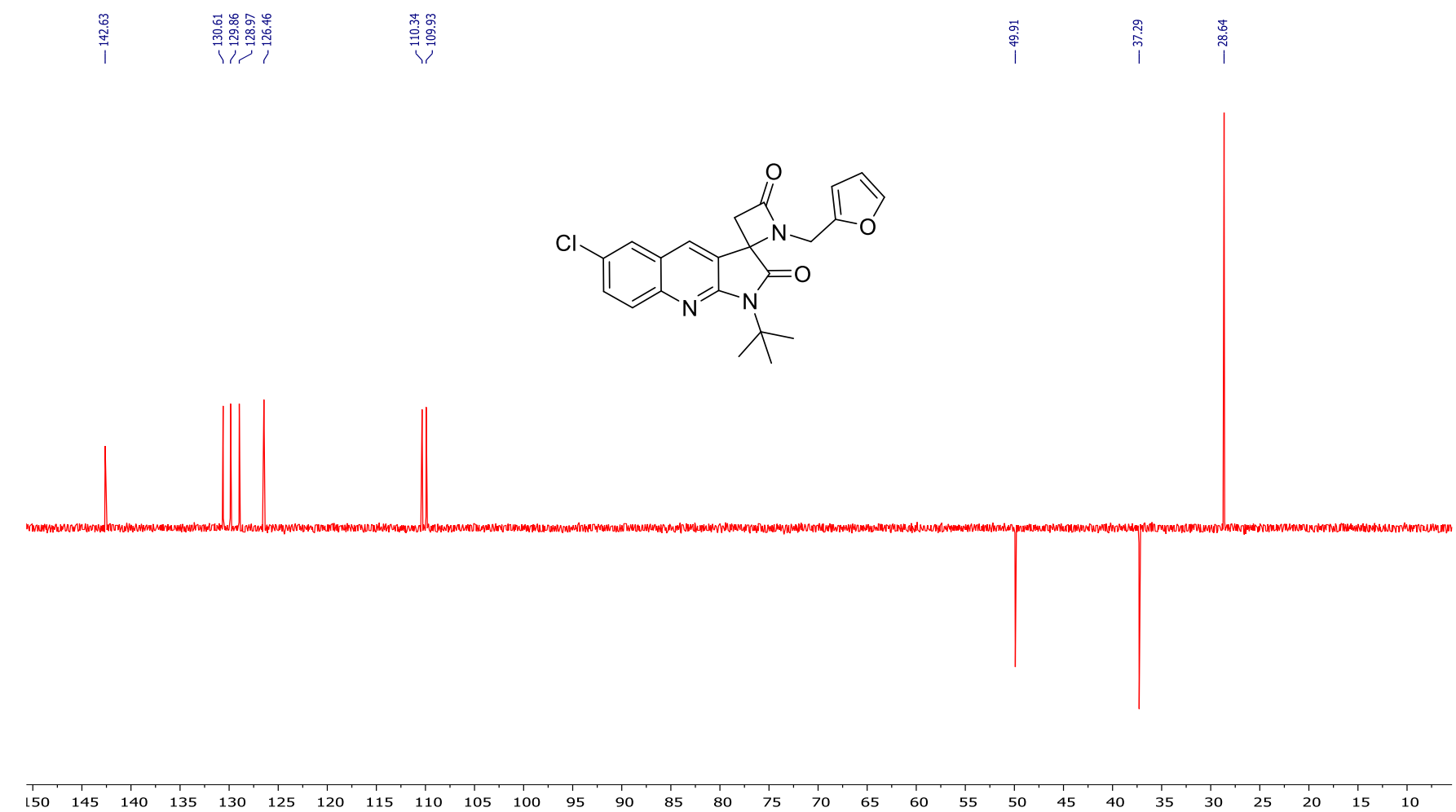

Figure S155: ${ }^{13} \mathrm{C}-\mathrm{NMR}$ (DEPT 135) of compound 6n (150 MHz, $\left.\mathrm{CDCl}_{3}\right)$

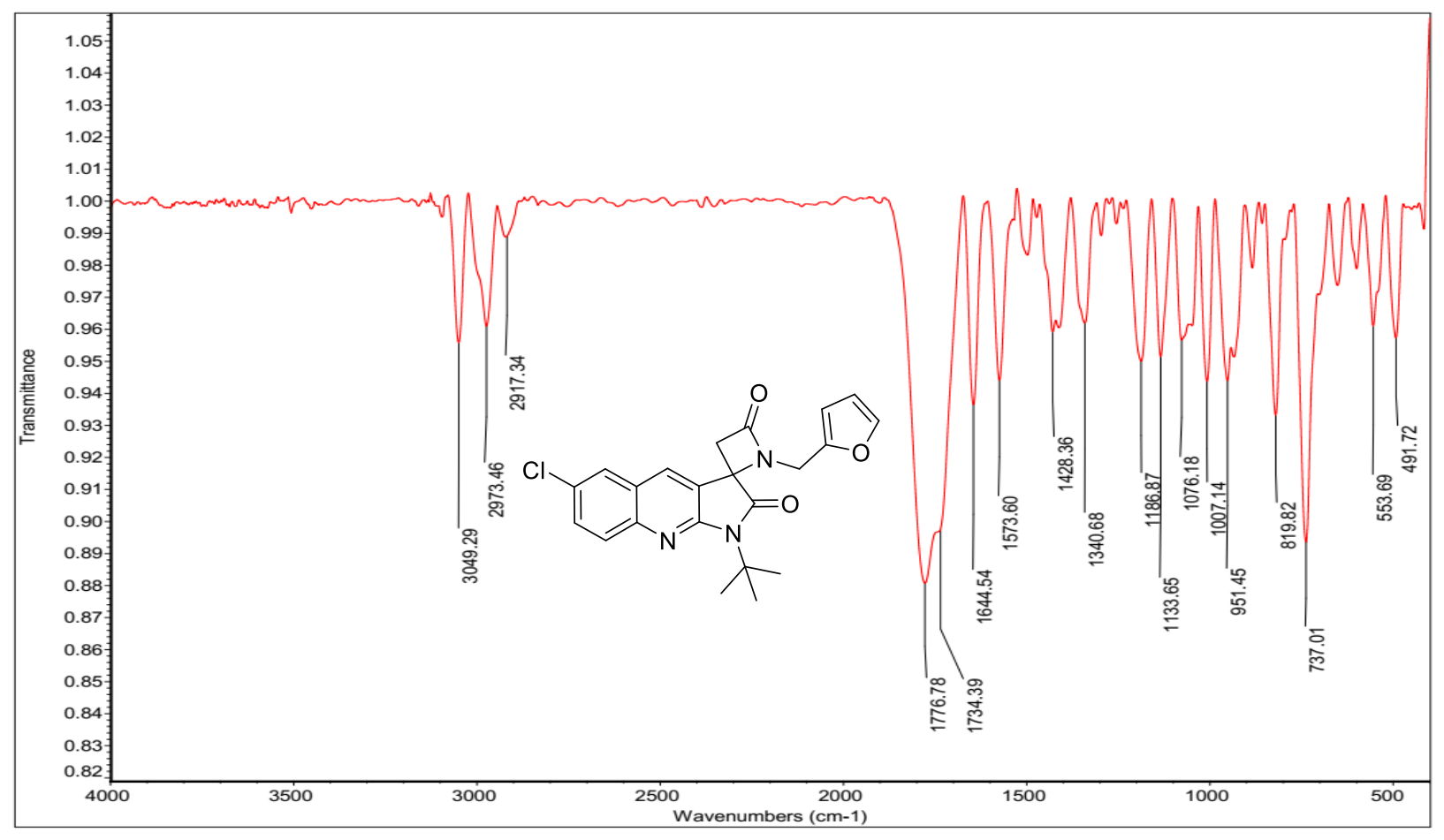

Figure S156: IR of compound $6 \mathbf{n}\left(\mathrm{KBr}, \mathrm{cm}^{-1}\right)$ 


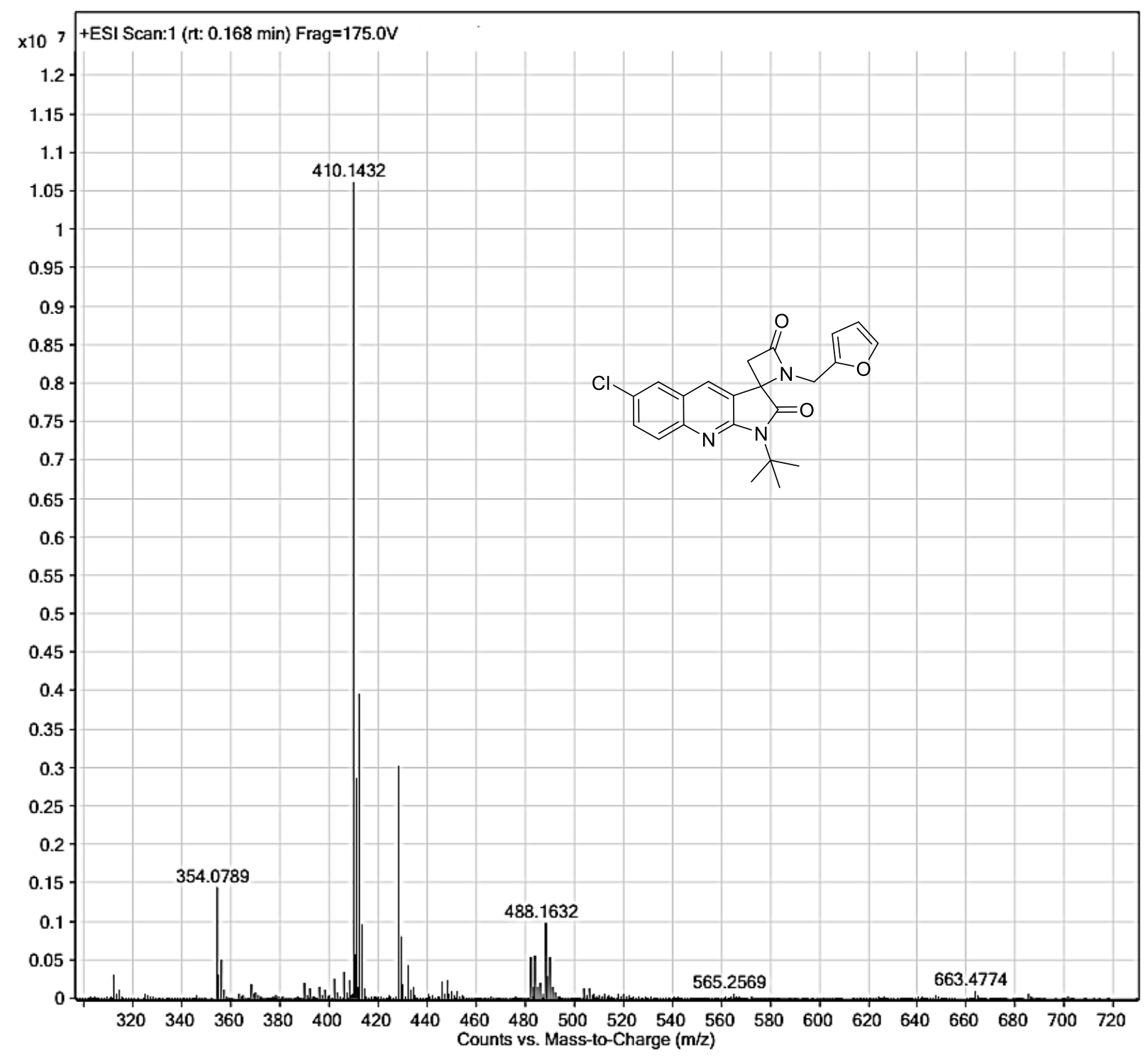

Figure S157: HRMS-ESI of 6 n with formula $\mathrm{C}_{22} \mathrm{H}_{20} \mathrm{ClN}_{3} \mathrm{O}_{3}$ and $[\mathrm{M}+\mathrm{H}]^{+} 410.1440$. 

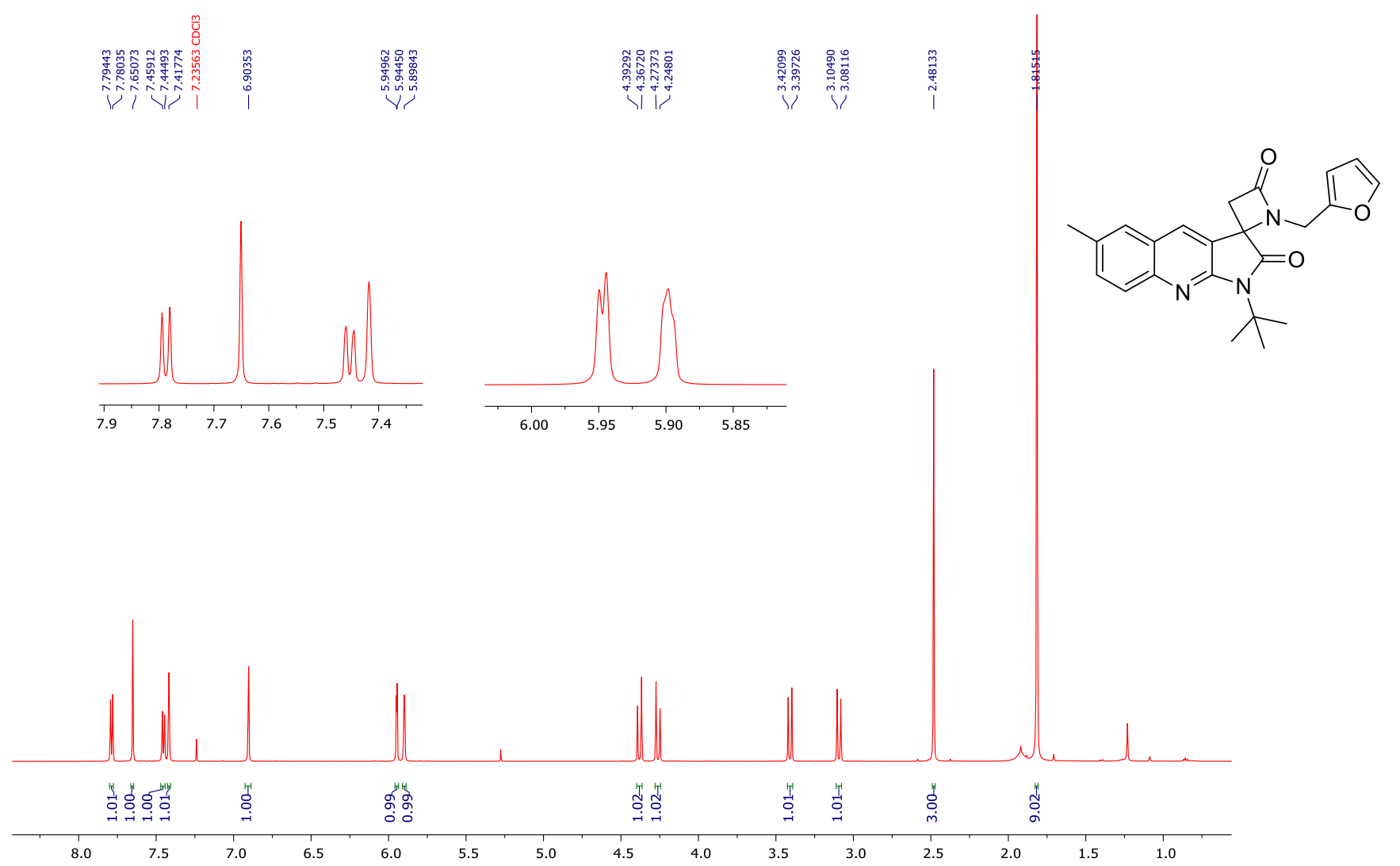

Figure S158: ${ }^{1} \mathrm{H}-\mathrm{NMR}$ of compound $60\left(600 \mathrm{MHz}, \mathrm{CDCl}_{3}\right)$
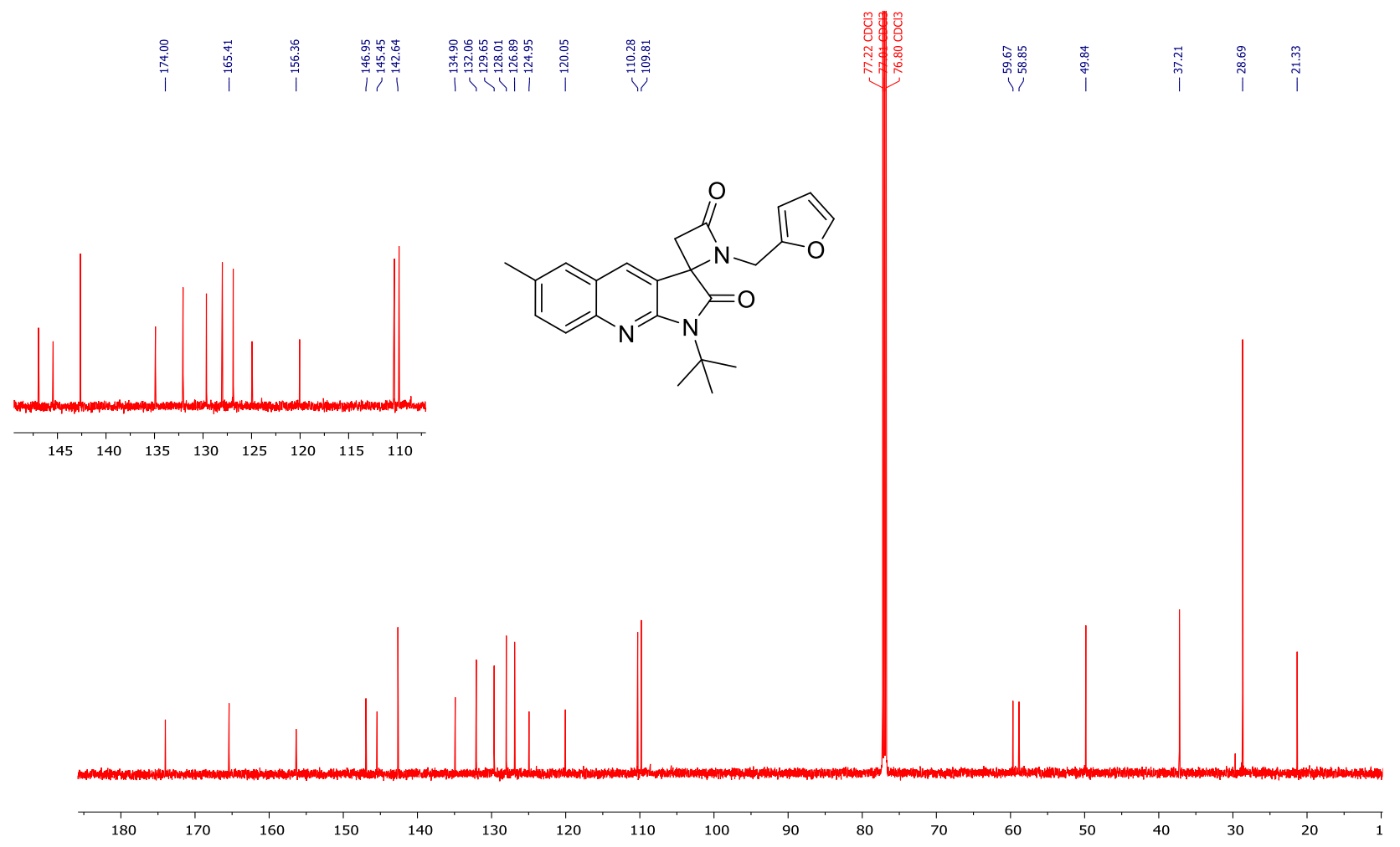

Figure S159: ${ }^{13} \mathrm{C}-\mathrm{NMR}$ of compound $60\left(150 \mathrm{MHz}, \mathrm{CDCl}_{3}\right)$ 


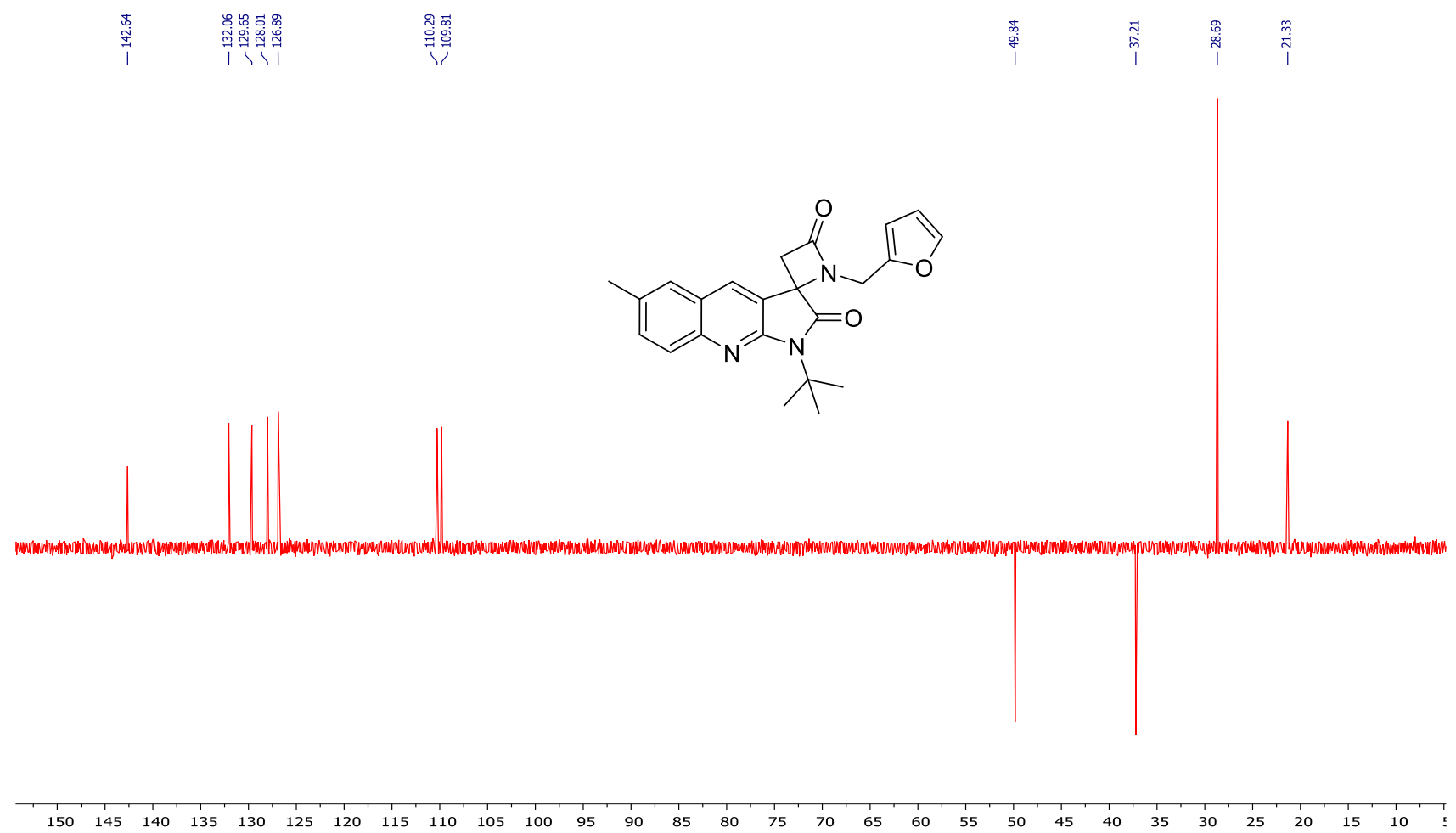

Figure S160: ${ }^{13} \mathrm{C}-\mathrm{NMR}$ (DEPT 135) of compound 60 (150 MHz, $\left.\mathrm{CDCl}_{3}\right)$

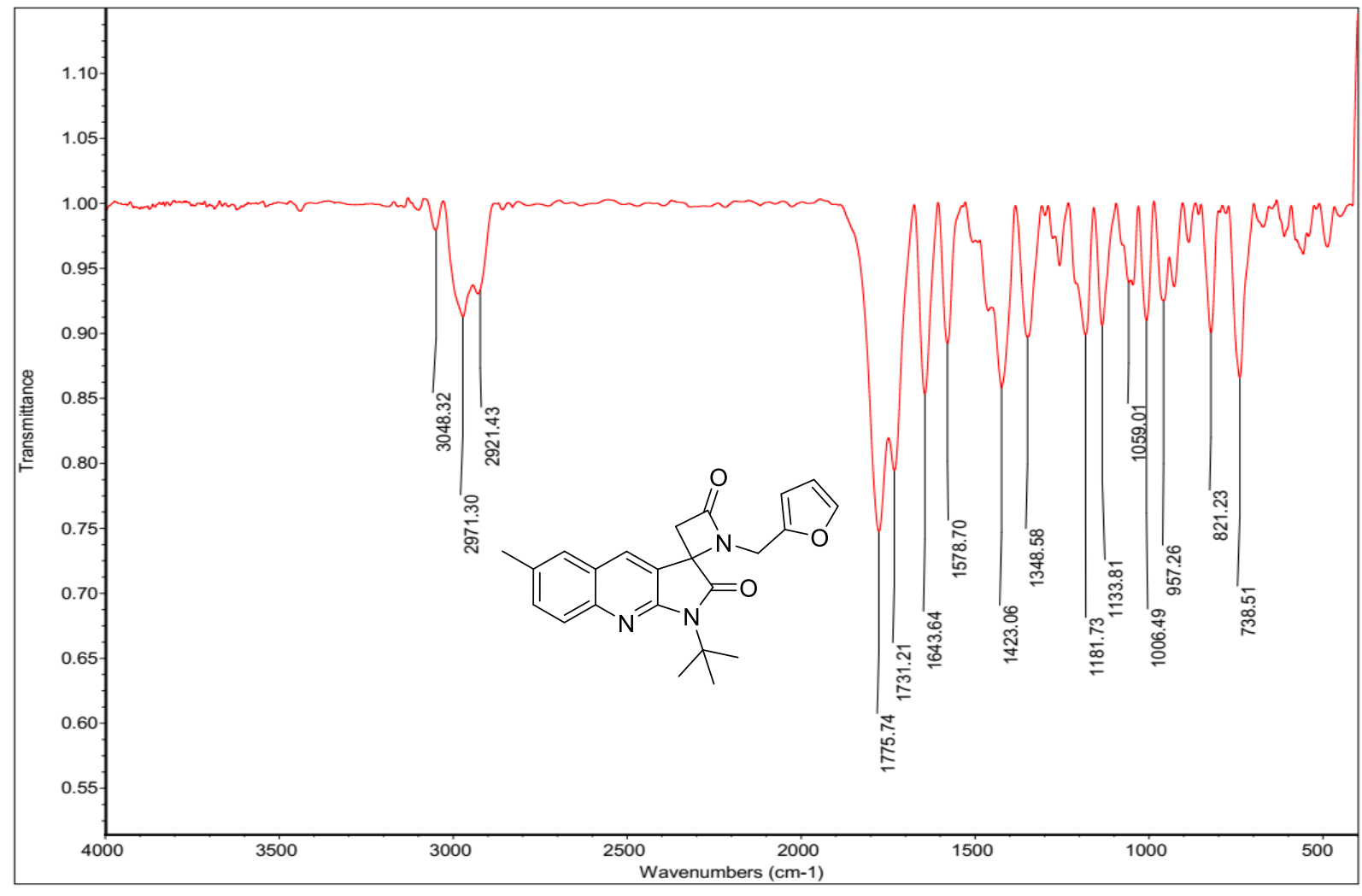

Figure S161: IR of compound $60\left(\mathrm{KBr}, \mathrm{cm}^{-1}\right)$ 


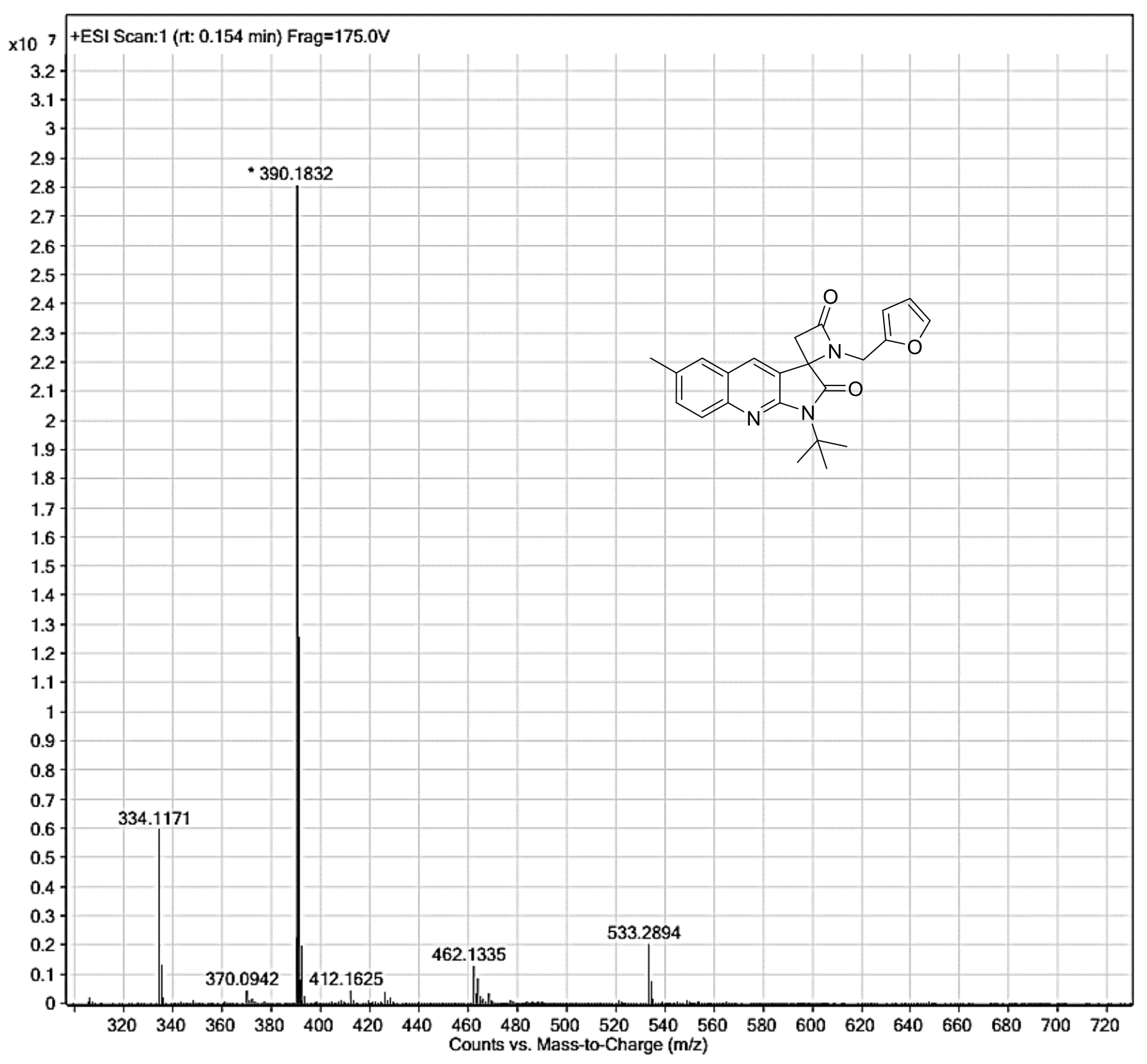

Figure S162: HRMS-ESI of 60 with formula $\mathrm{C}_{23} \mathrm{H}_{23} \mathrm{~N}_{3} \mathrm{O}_{3}$ and $[\mathrm{M}+\mathrm{H}]^{+} 390.1823$, and $[\mathrm{M}+\mathrm{Na}]^{+}$ 412.1617. 

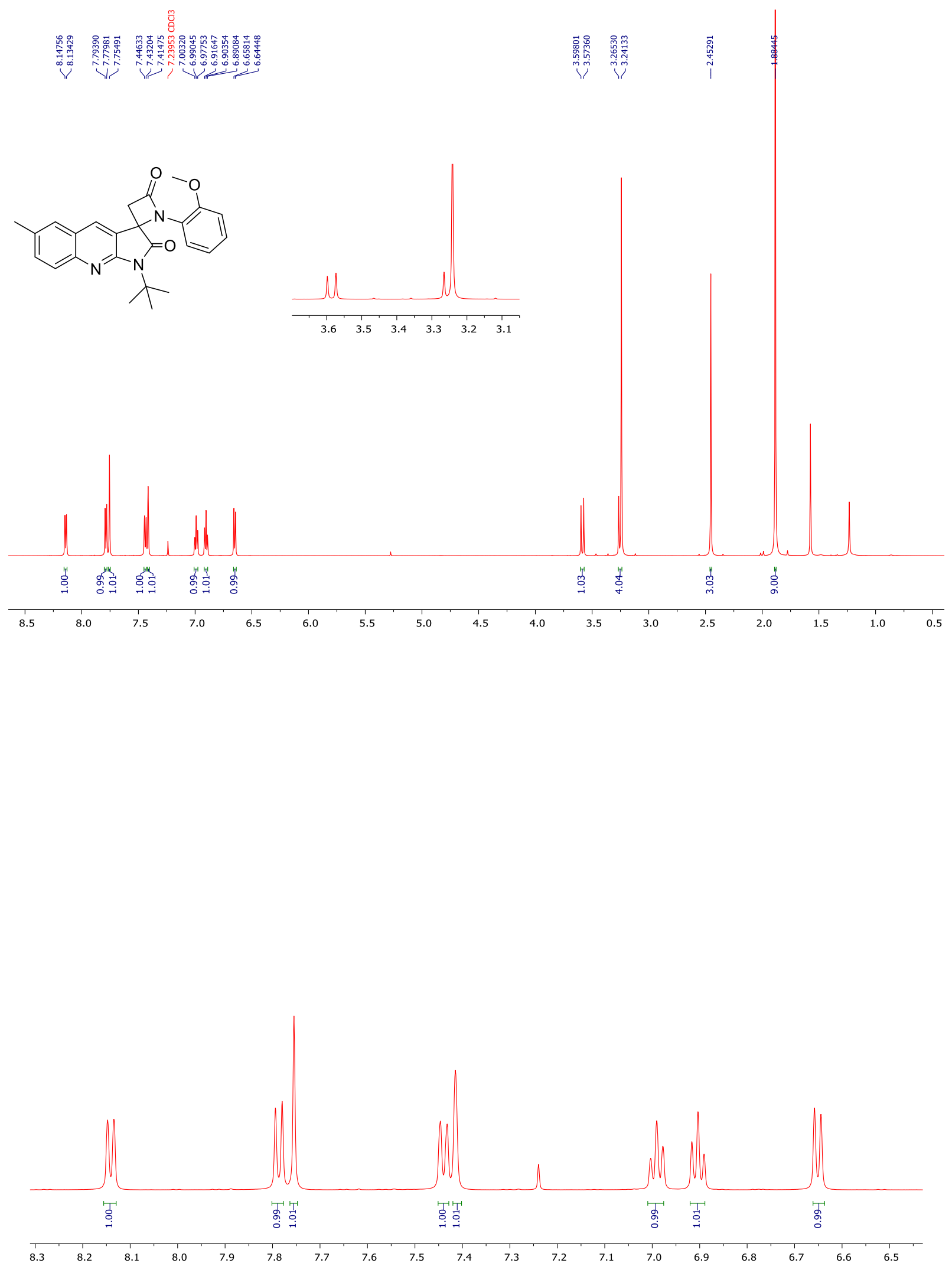

Figure S163: ${ }^{1} \mathrm{H}-\mathrm{NMR}$ of compound $\mathbf{6 p}\left(600 \mathrm{MHz}, \mathrm{CDCl}_{3}\right)$ 


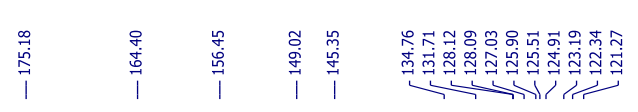
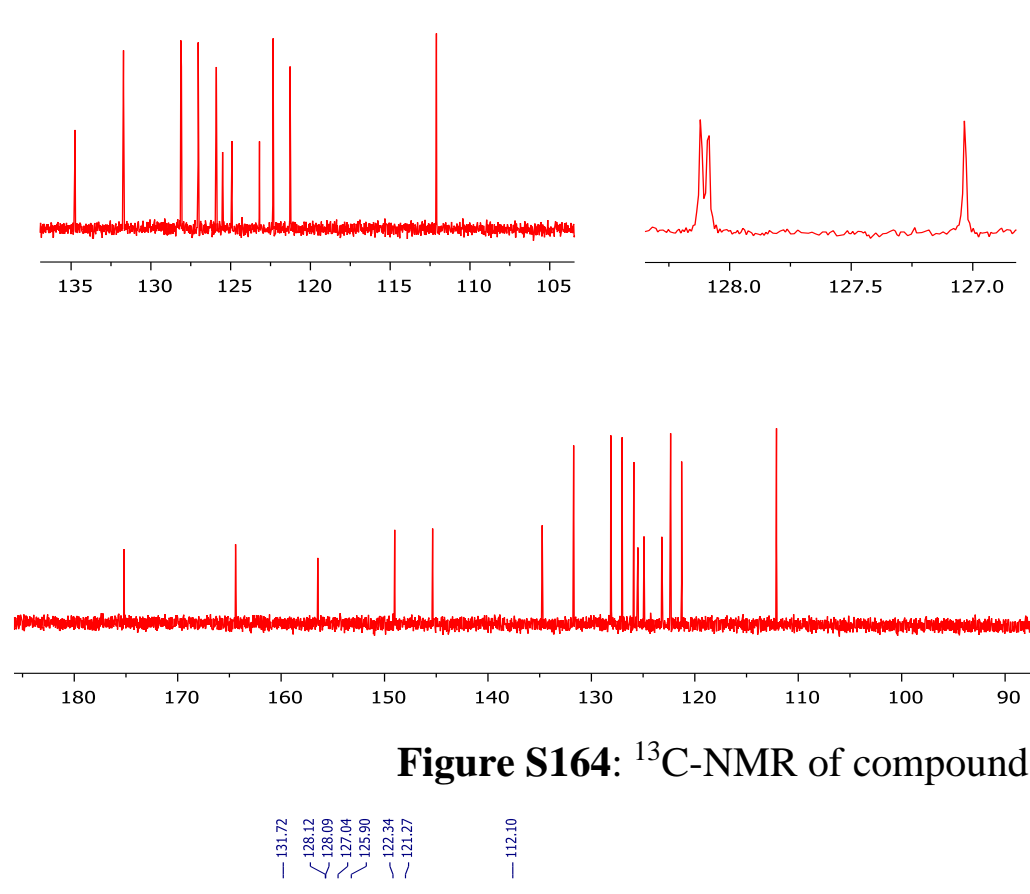

Figure

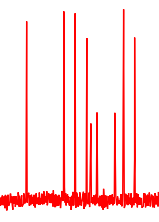

130

120

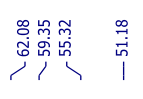

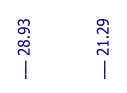

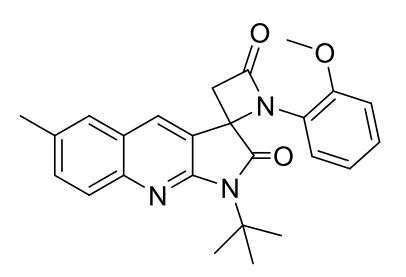

Whaning
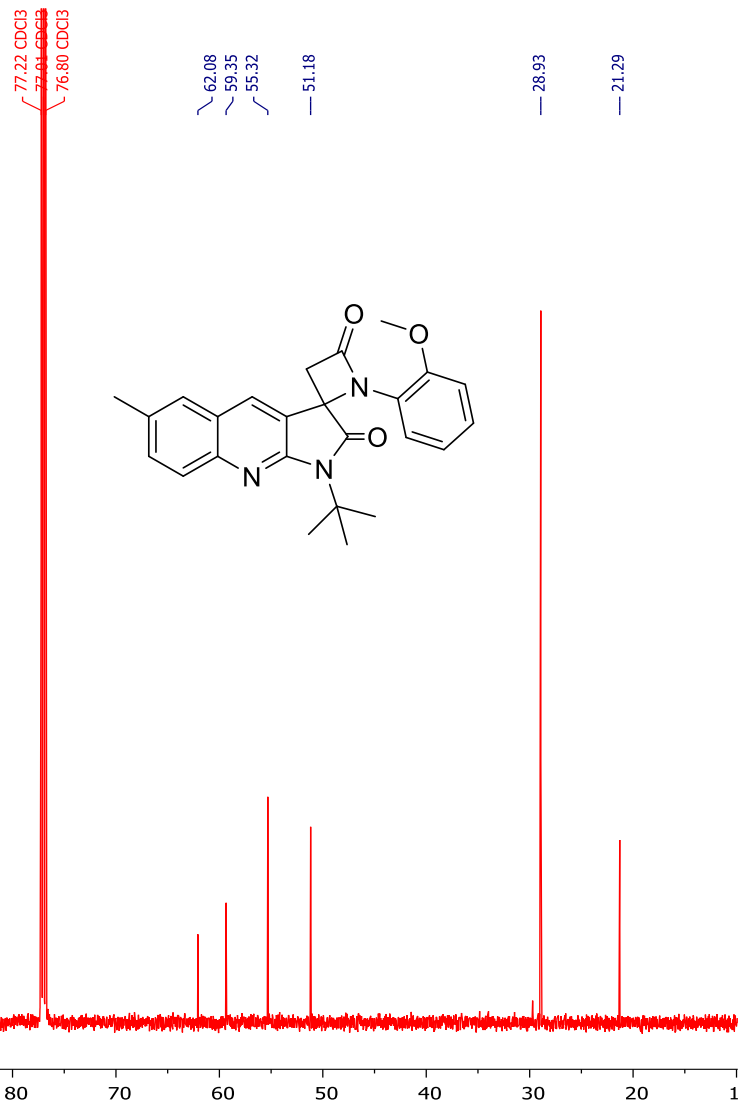

$\begin{array}{llll}10 & 100 & 90 & 80\end{array}$

70
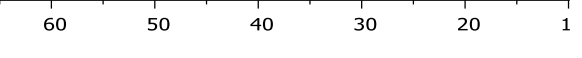

S164: ${ }^{13} \mathrm{C}-\mathrm{NMR}$ of compound $\mathbf{6 p}\left(150 \mathrm{MHz}, \mathrm{CDCl}_{3}\right)$

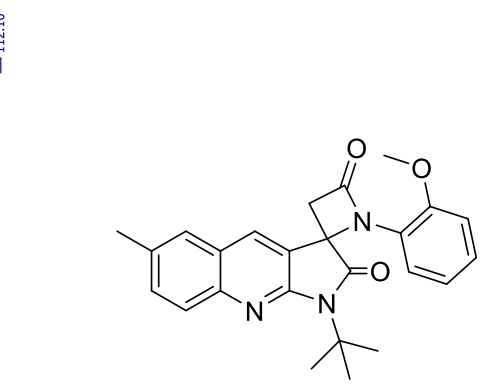

藋
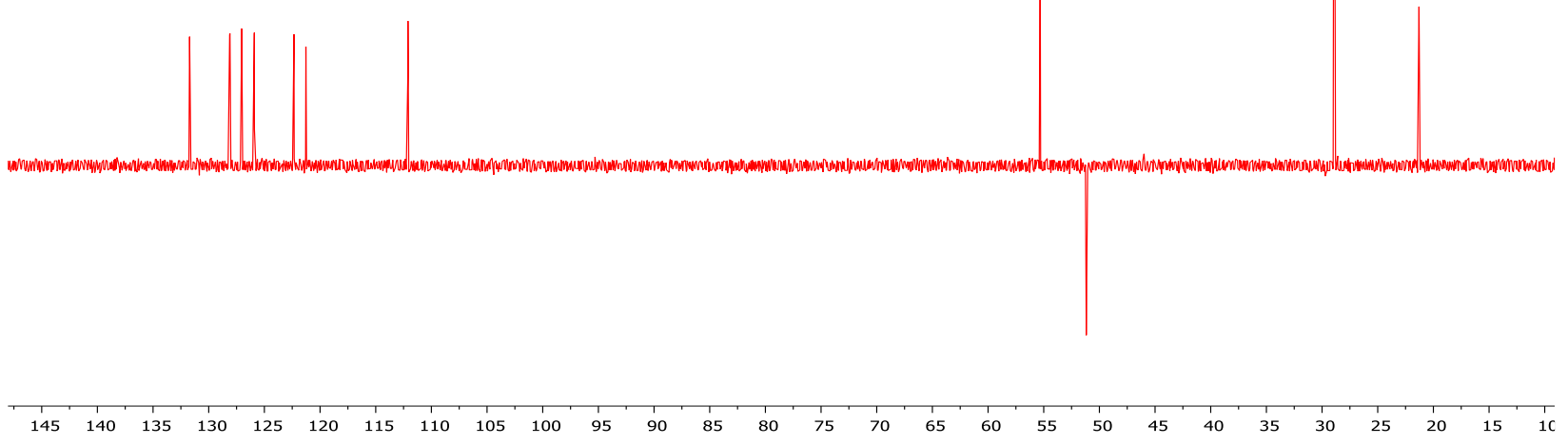

Figure S165: ${ }^{13} \mathrm{C}-\mathrm{NMR}$ (DEPT 135) of compound $\mathbf{6 p}\left(150 \mathrm{MHz}, \mathrm{CDCl}_{3}\right)$

S97 


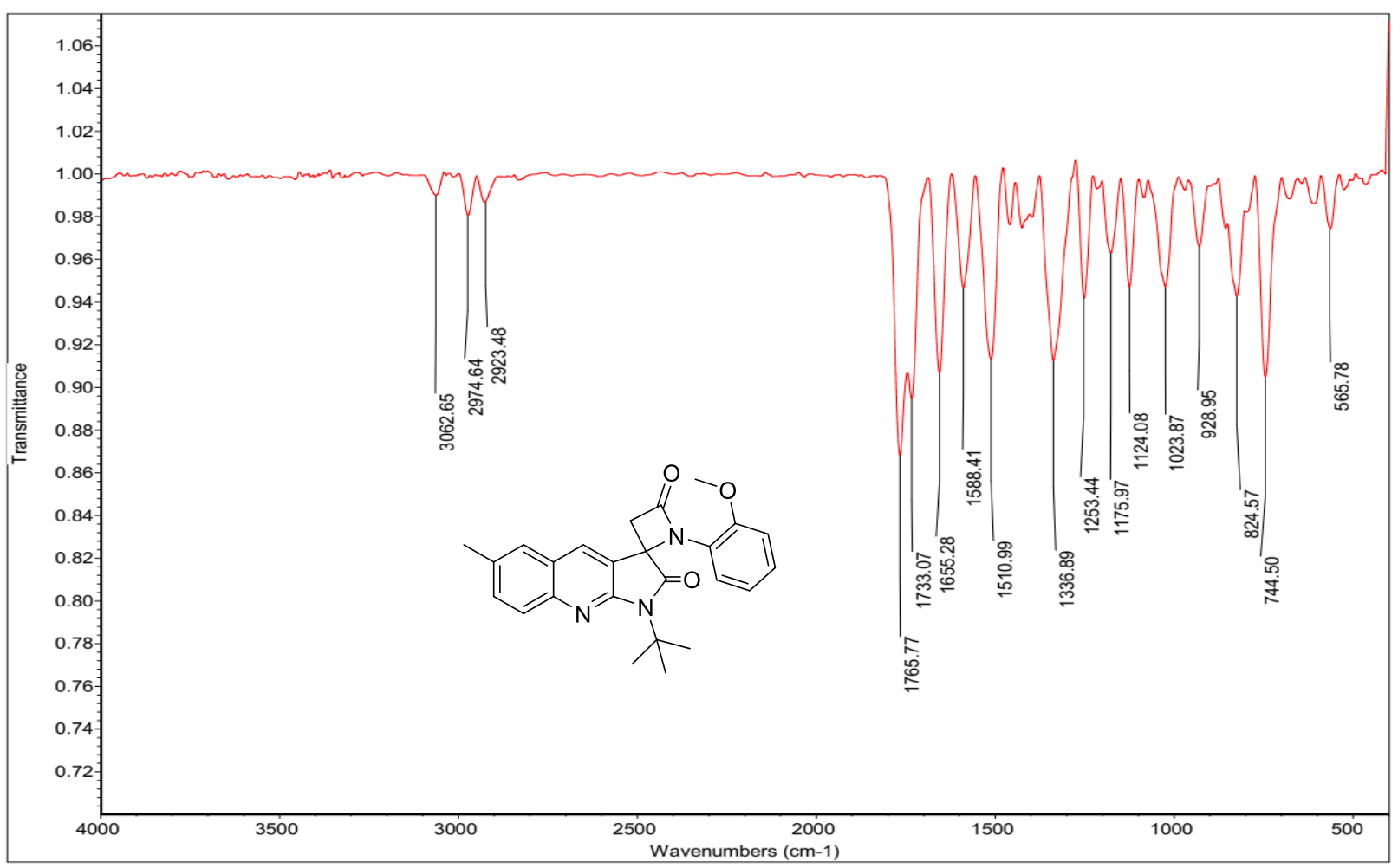

Figure S166: IR of compound $\mathbf{6 p}\left(\mathrm{KBr}, \mathrm{cm}^{-1}\right)$

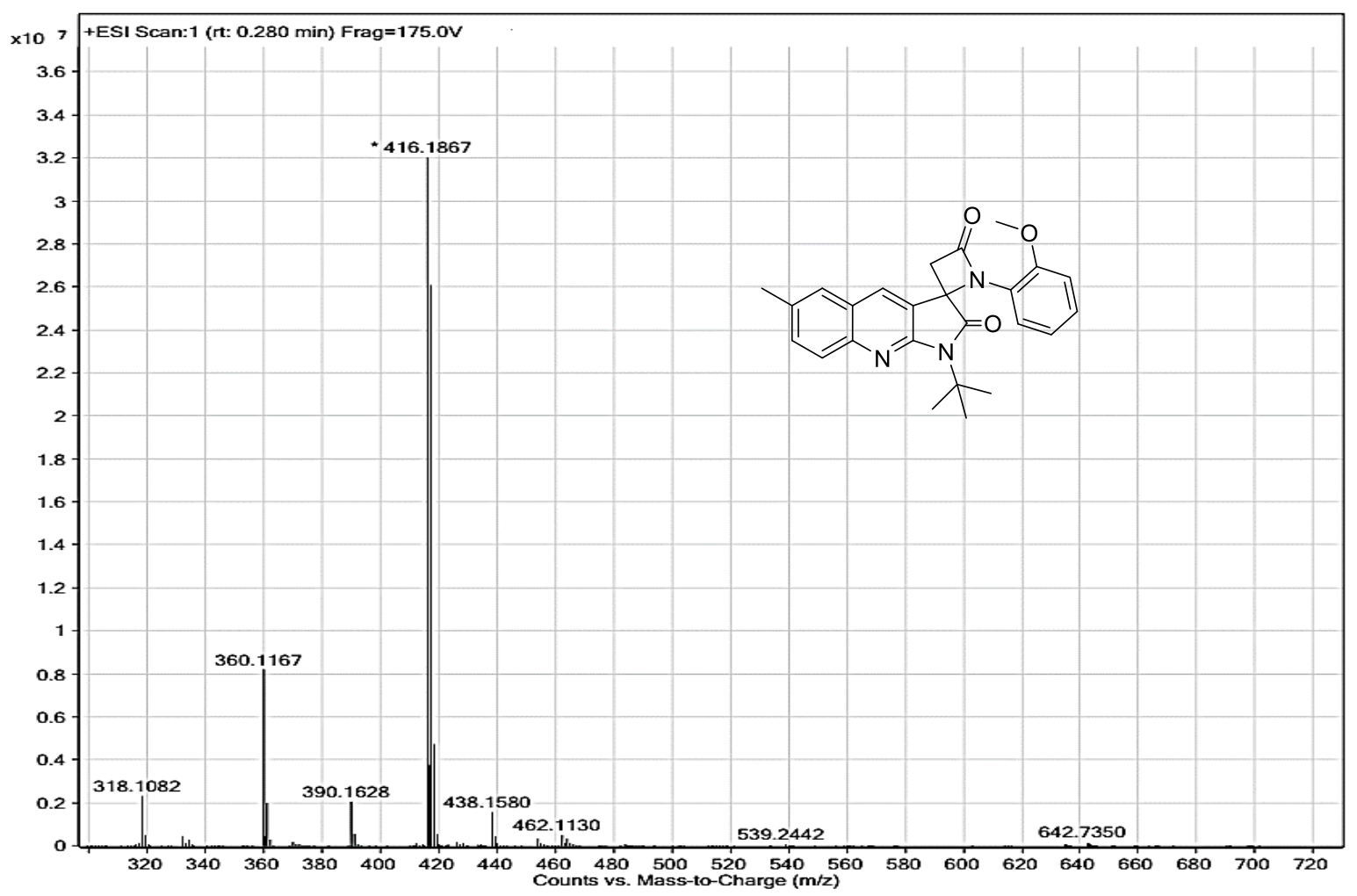

Figure S167: HRMS-ESI of $\mathbf{6 p}$ with formula $\mathrm{C}_{25} \mathrm{H}_{25} \mathrm{~N}_{3} \mathrm{O}_{3}$ and $[\mathrm{M}+\mathrm{H}]^{+} 416.1874$, and $[\mathrm{M}+\mathrm{Na}]^{+}$ 438.1590 . 


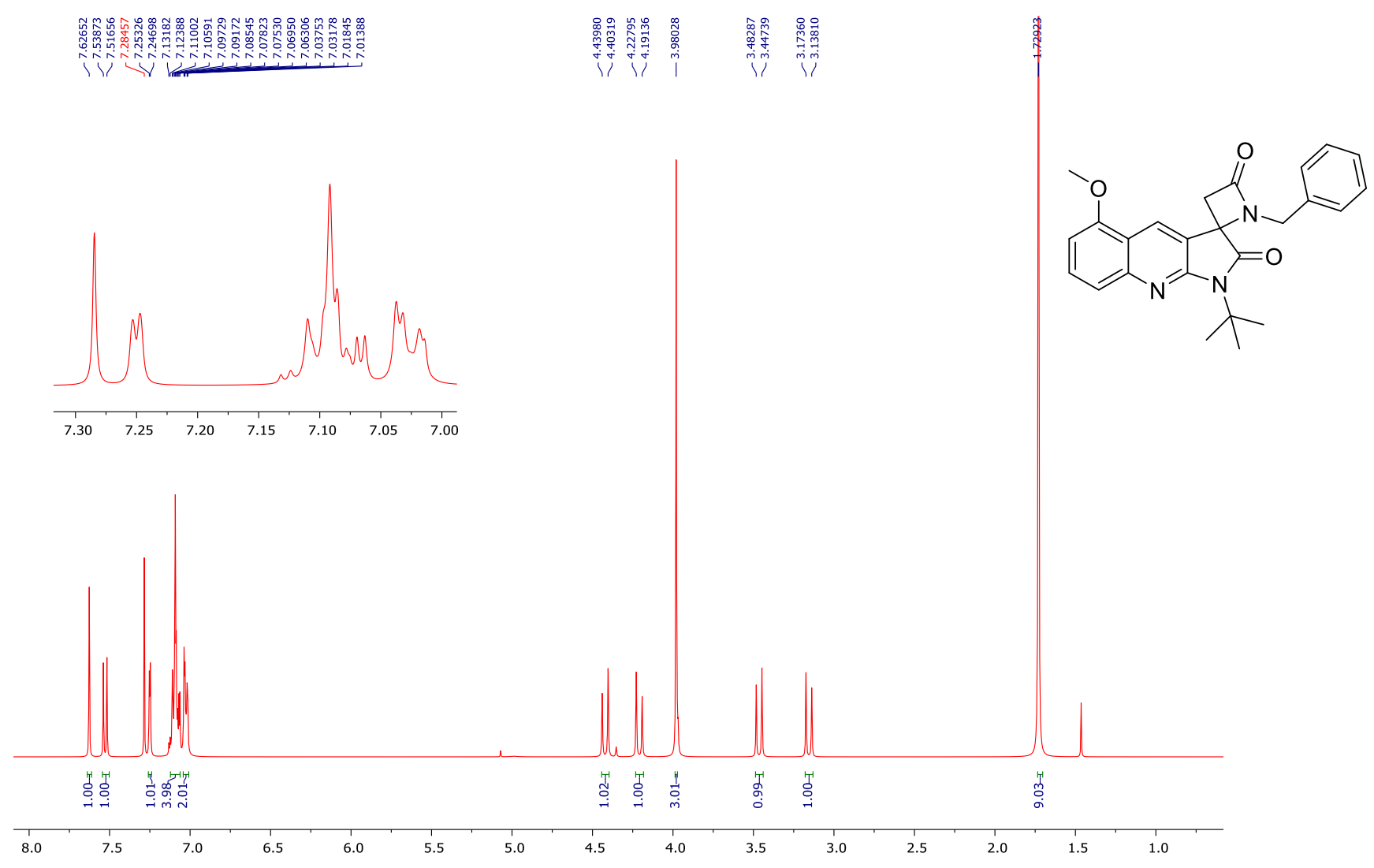

Figure S168: ${ }^{1} \mathrm{H}-\mathrm{NMR}$ of compound $\mathbf{6 q}\left(400 \mathrm{MHz}, \mathrm{CDCl}_{3}\right)$

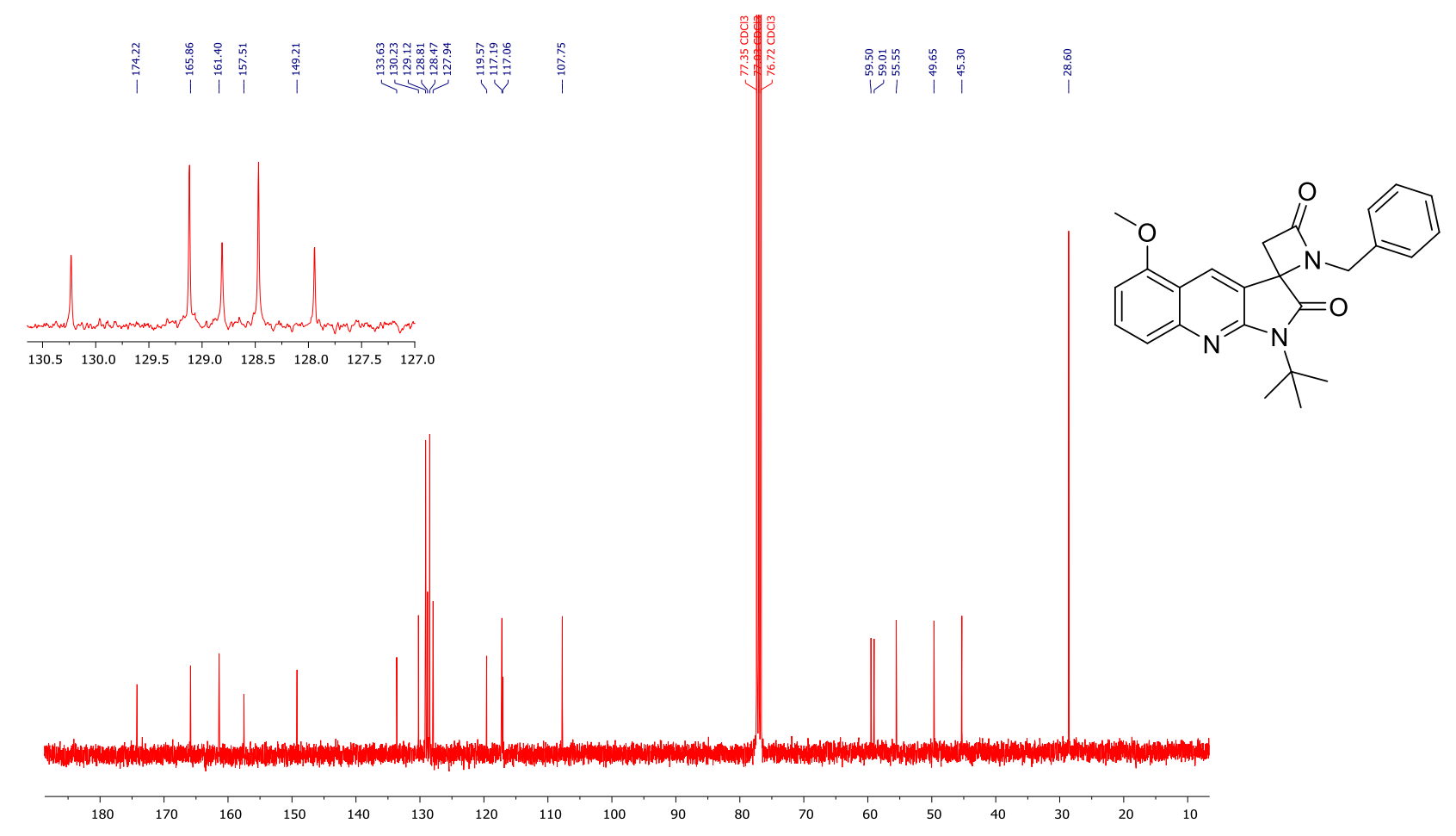

Figure S169: ${ }^{13} \mathrm{C}-\mathrm{NMR}$ of compound $\mathbf{6 q}\left(100 \mathrm{MHz}, \mathrm{CDCl}_{3}\right)$ 


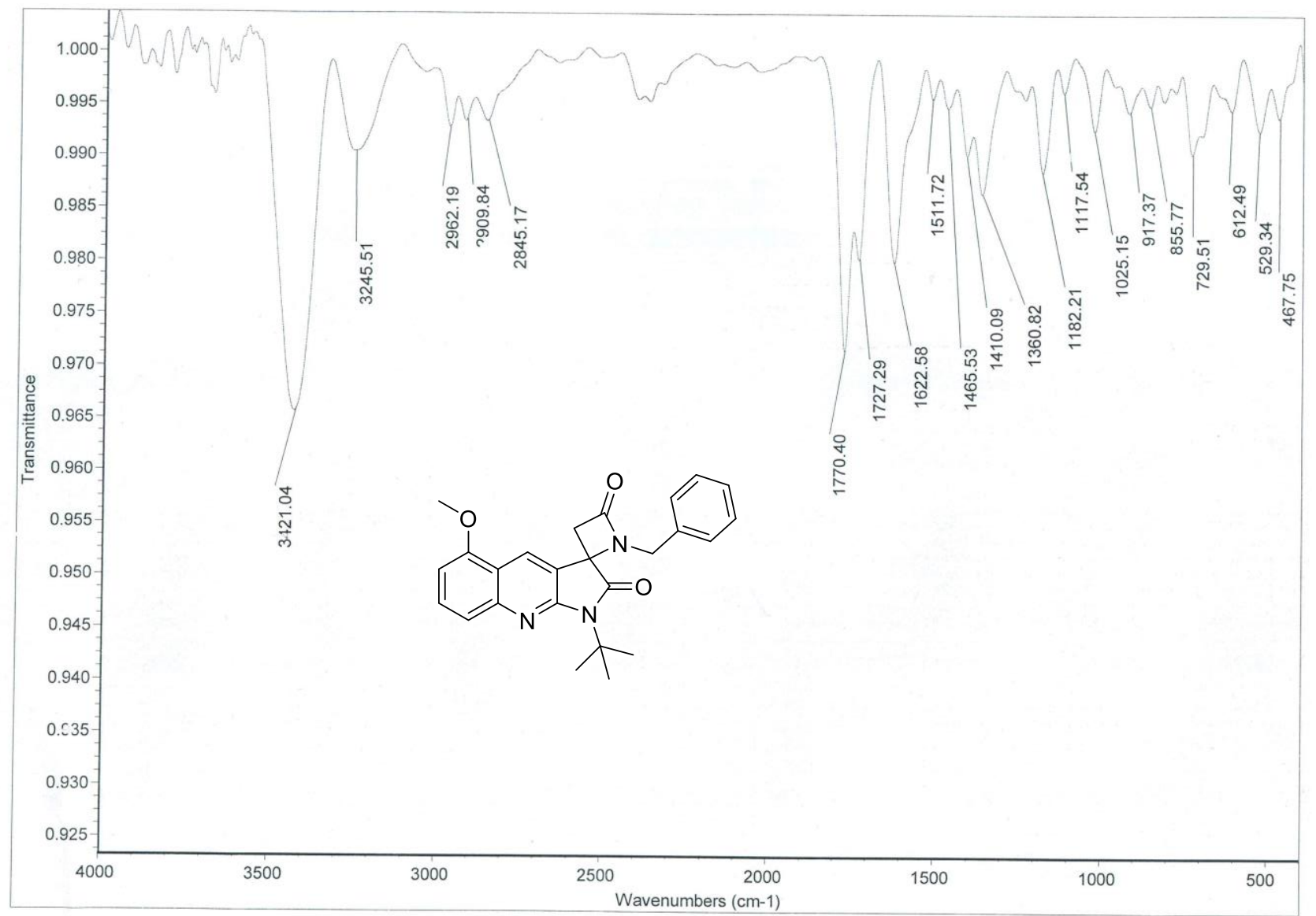

Figure S170: IR of compound $\mathbf{6 q}\left(\mathrm{KBr}, \mathrm{cm}^{-1}\right)$ 


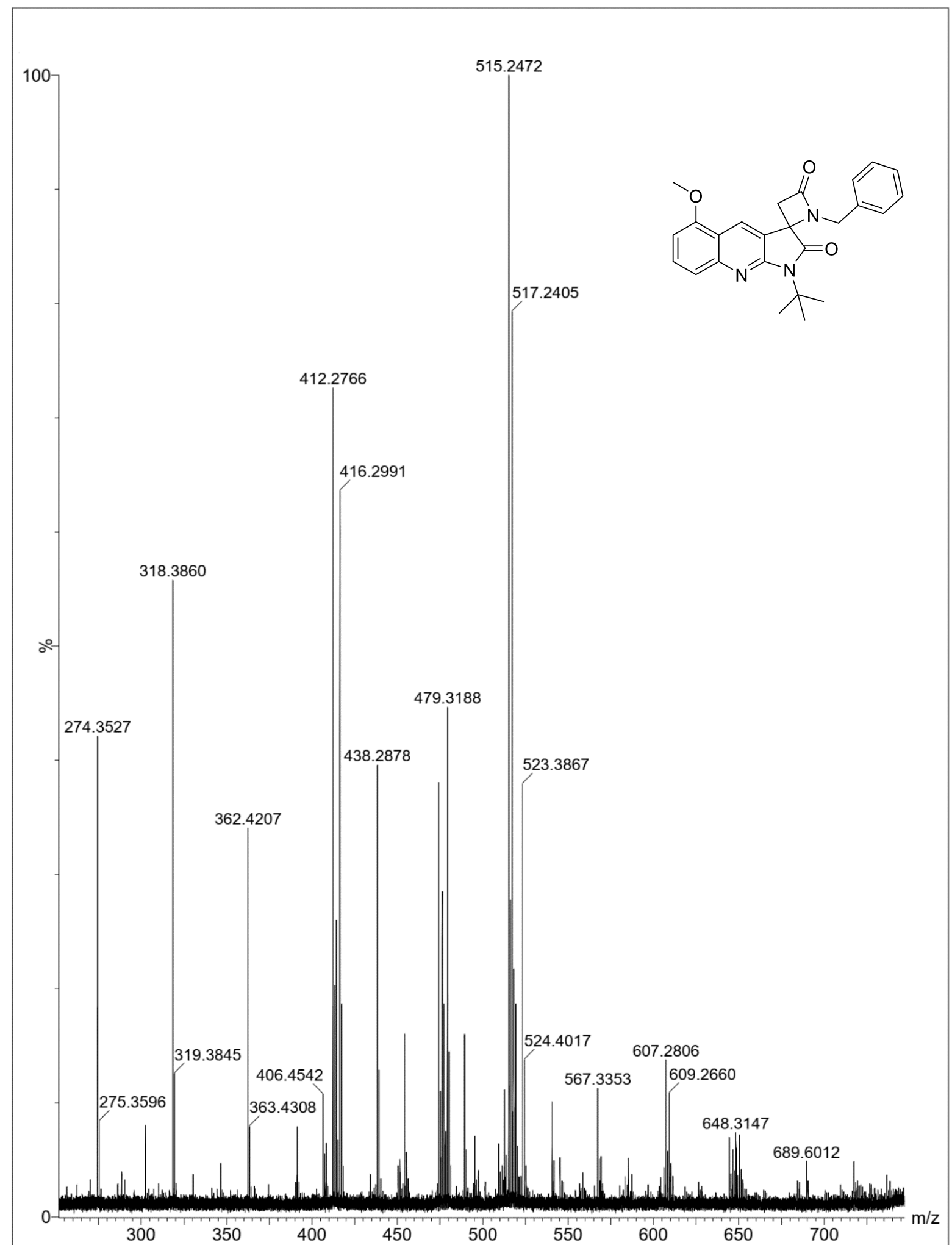

Figure S171: ESI-MS (+) of compound $\mathbf{6 q}$ with formula $\mathrm{C}_{25} \mathrm{H}_{25} \mathrm{~N}_{3} \mathrm{O}_{3}$ and $[\mathrm{M}+\mathrm{H}]^{+} 416.2991$. 


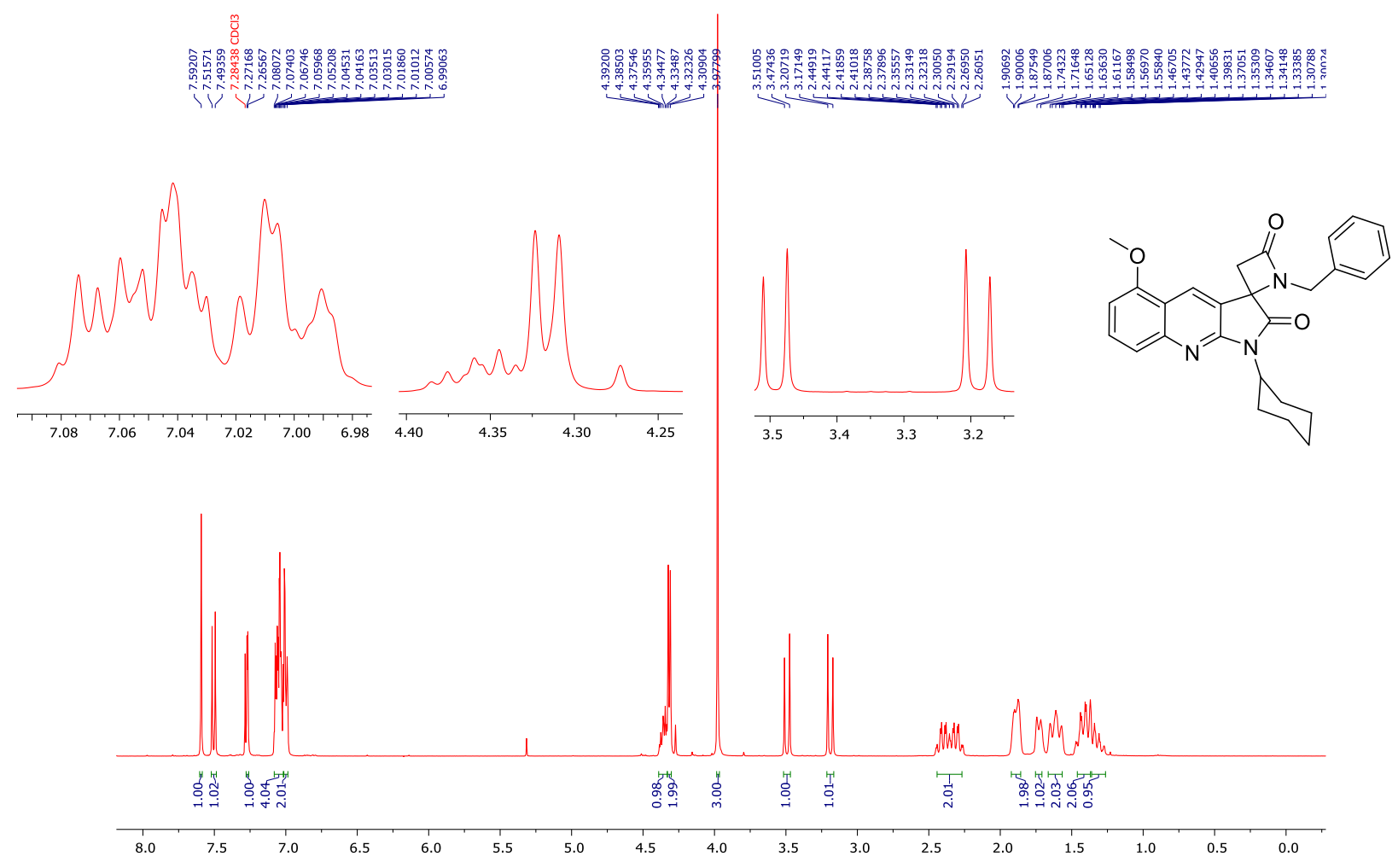

Figure S172: ${ }^{1} \mathrm{H}-\mathrm{NMR}$ of compound $6 \mathbf{r}\left(400 \mathrm{MHz}, \mathrm{CDCl}_{3}\right)$

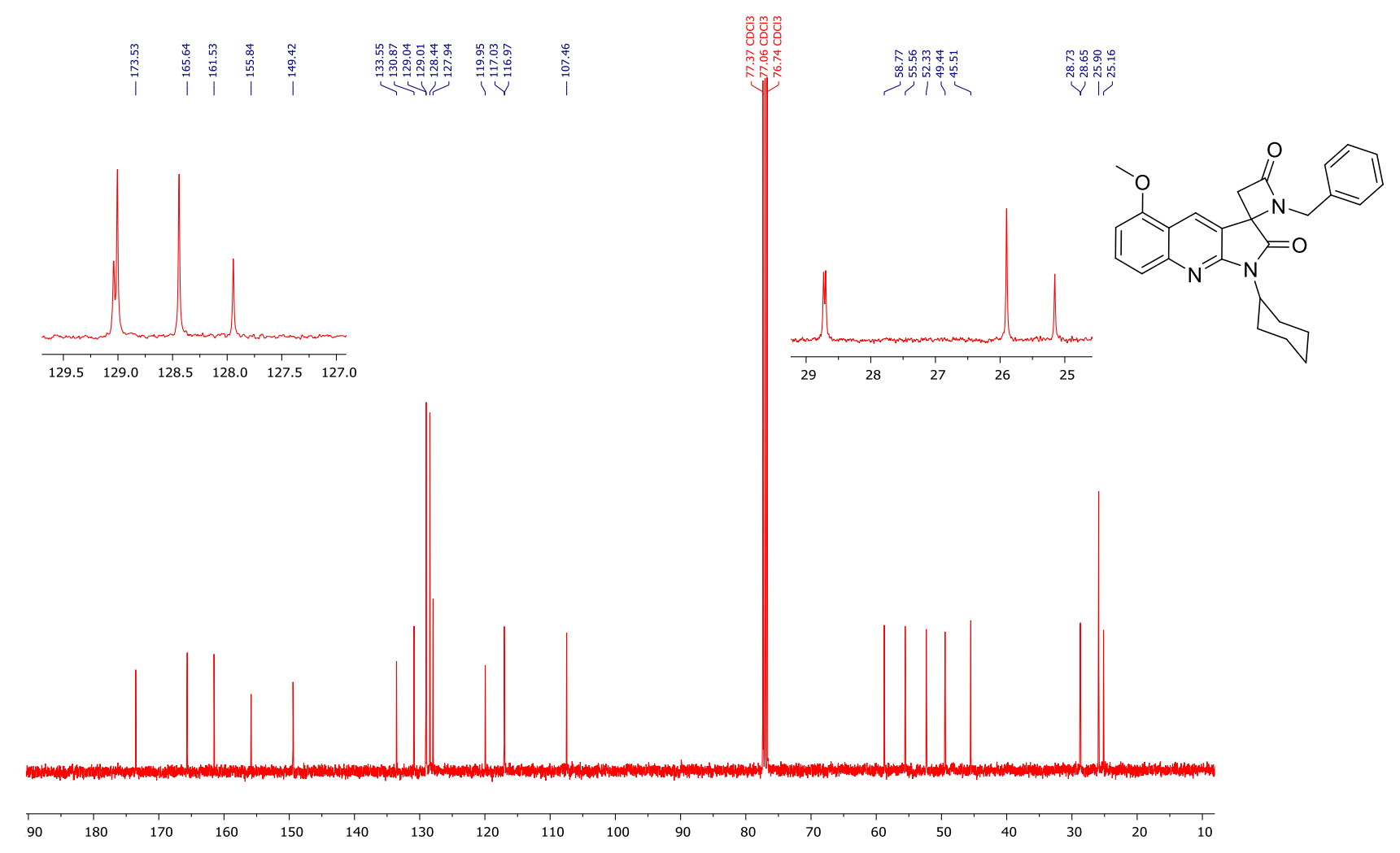

Figure S173: ${ }^{13} \mathrm{C}-\mathrm{NMR}$ of compound $6 \mathbf{r}\left(100 \mathrm{MHz}, \mathrm{CDCl}_{3}\right)$ 


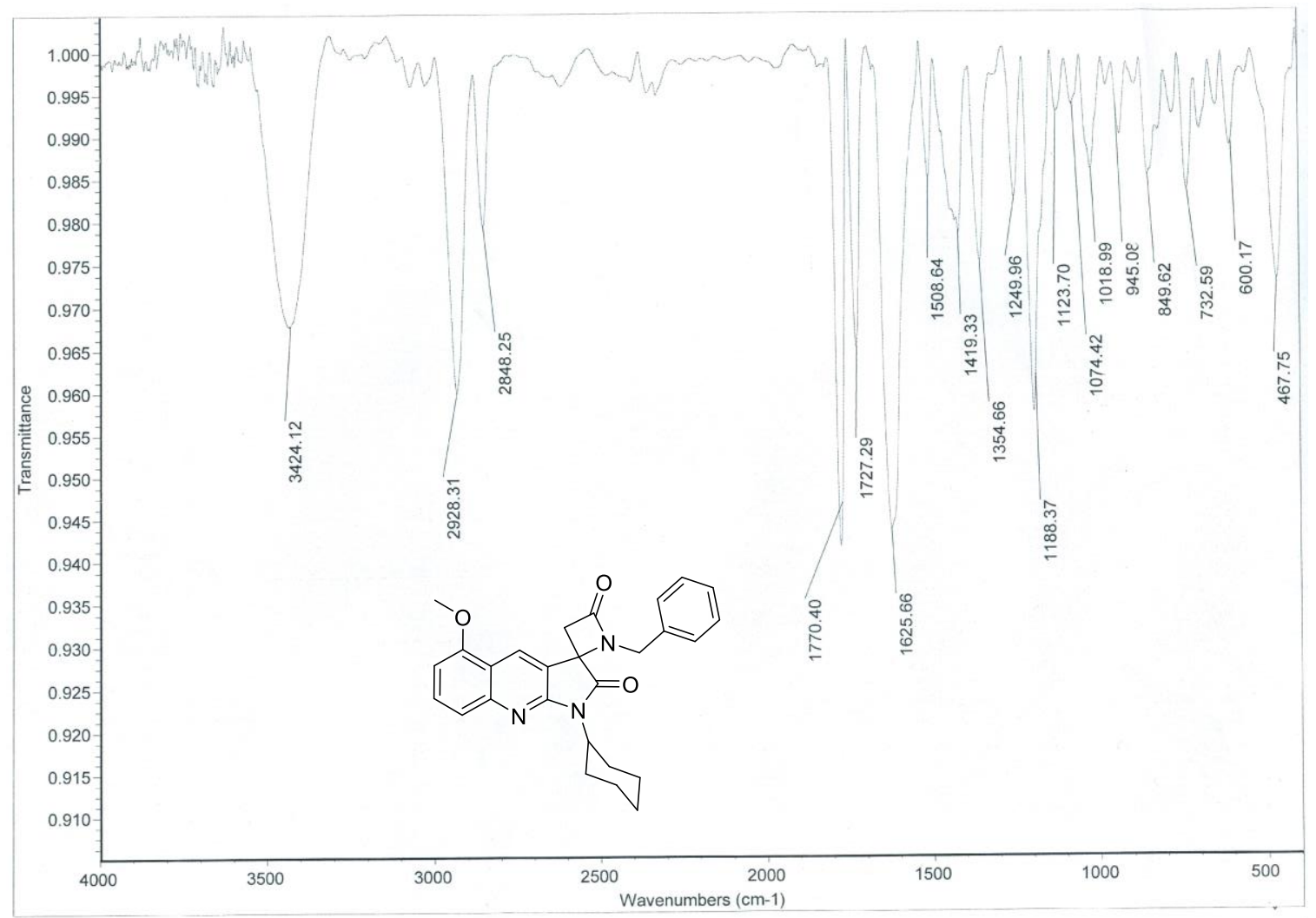

Figure S174: IR of compound $6 \mathbf{r}\left(\mathrm{KBr}, \mathrm{cm}^{-1}\right)$ 


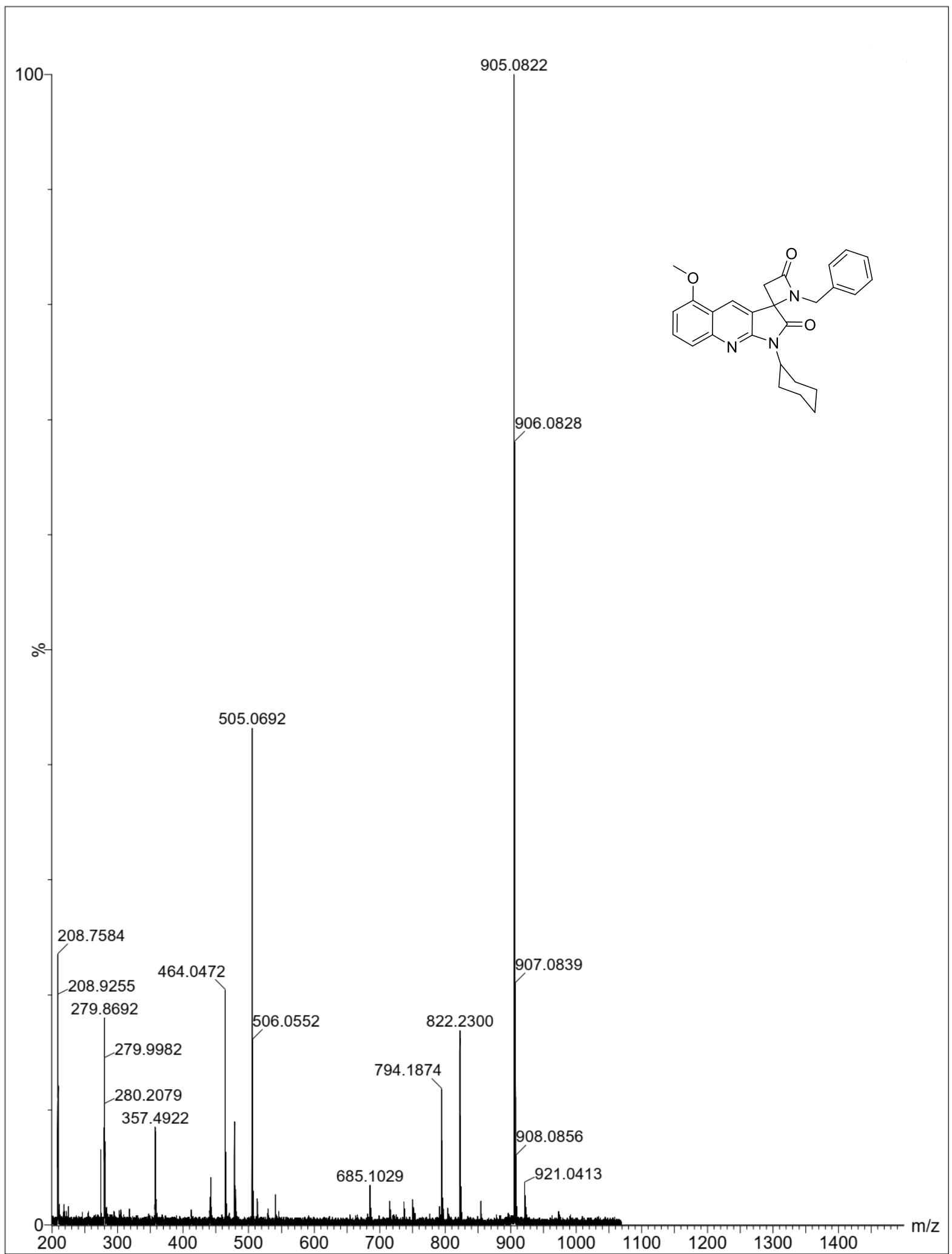

Figure S175: ESI-MS (+) of compound 6r with formula $\mathrm{C}_{27} \mathrm{H}_{27} \mathrm{~N}_{3} \mathrm{O}_{3}$ and $[\mathrm{M}+\mathrm{Na}]^{+} 464.0472$. 

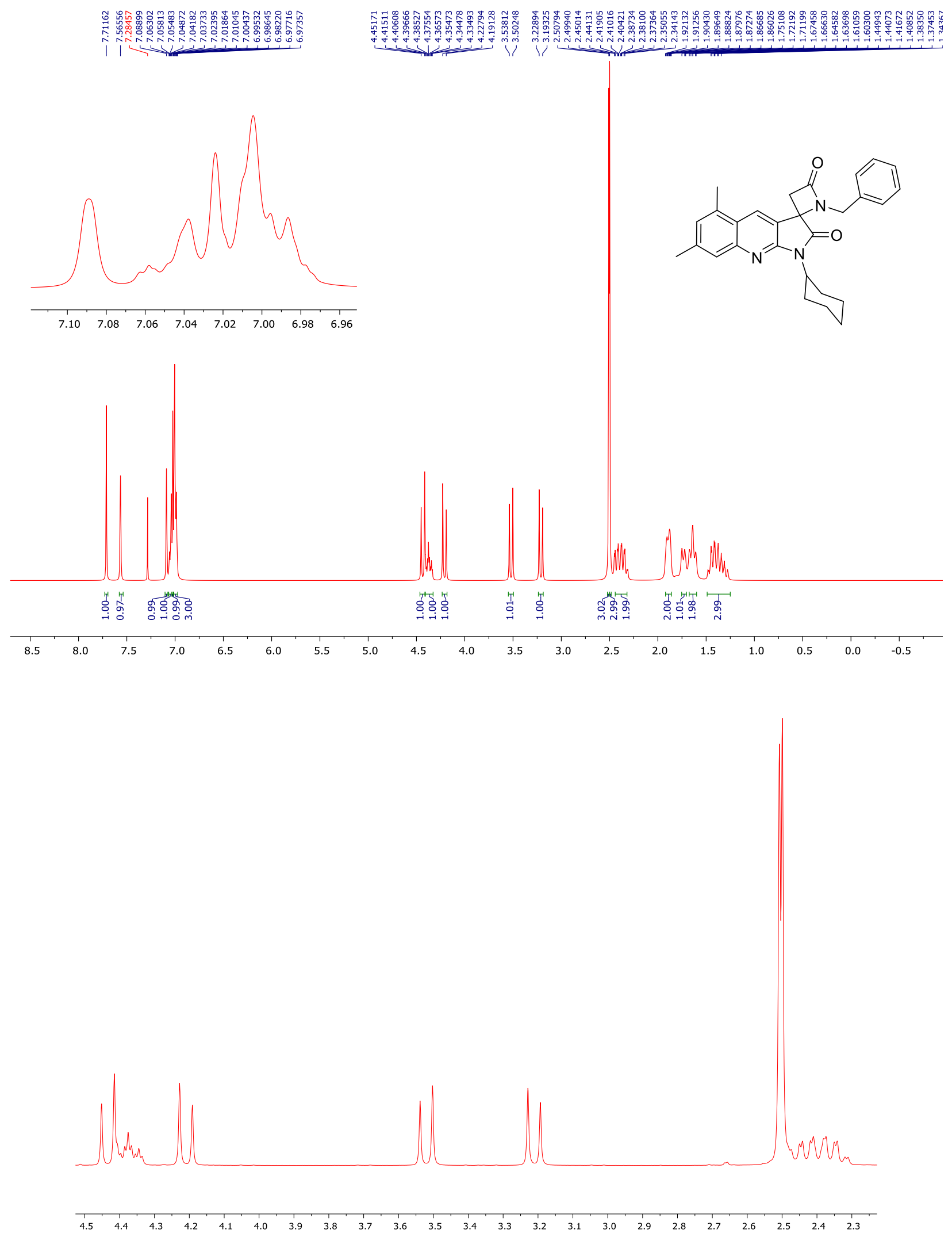

Figure S176: ${ }^{1} \mathrm{H}-\mathrm{NMR}$ of compound $\mathbf{6 s}\left(400 \mathrm{MHz}, \mathrm{CDCl}_{3}\right)$ 


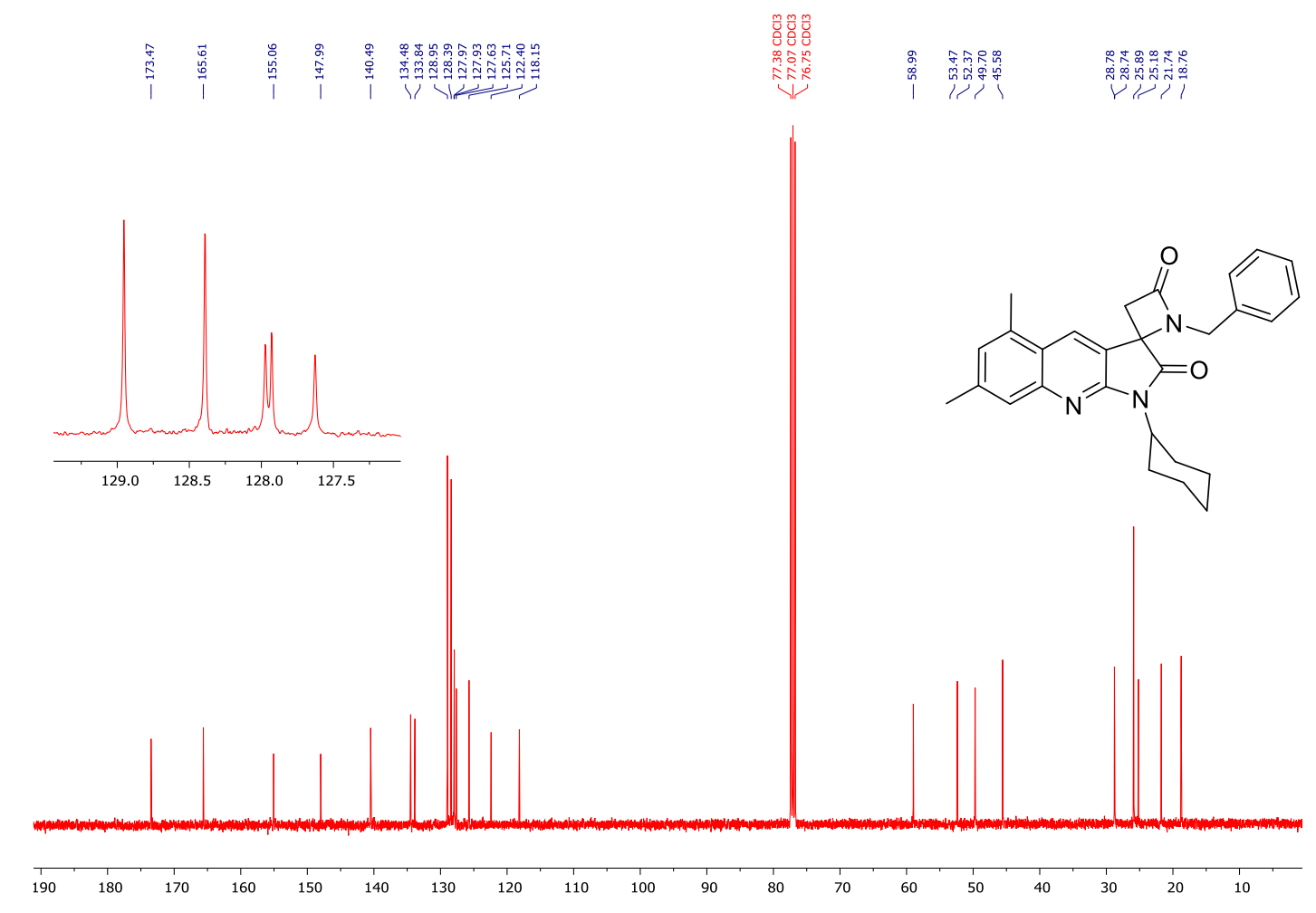

Figure S177: ${ }^{13} \mathrm{C}-\mathrm{NMR}$ of compound $\mathbf{6 s}\left(100 \mathrm{MHz}, \mathrm{CDCl}_{3}\right)$

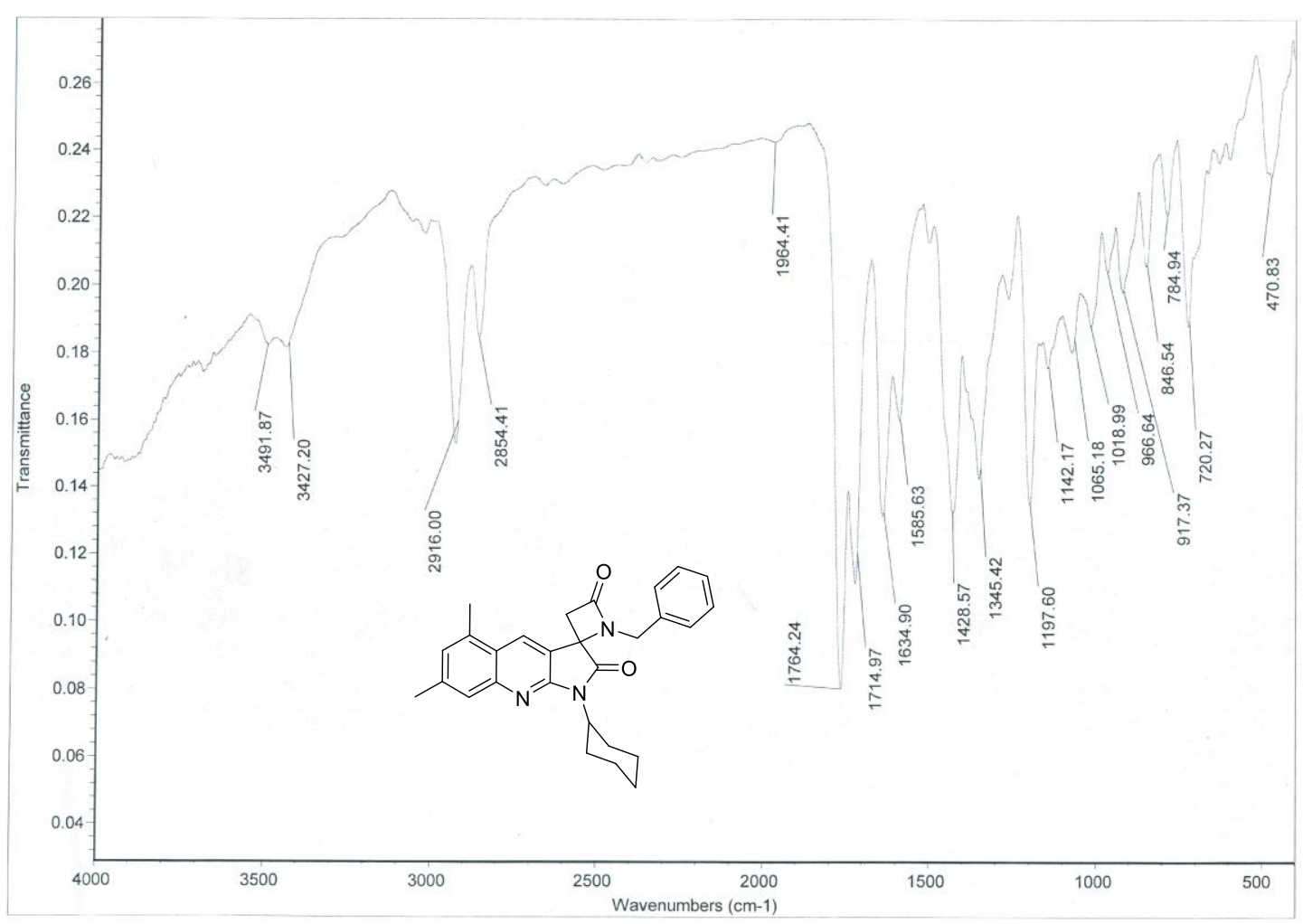

Figure S178: IR of compound $6 \mathbf{s}\left(\mathrm{KBr}, \mathrm{cm}^{-1}\right)$ 


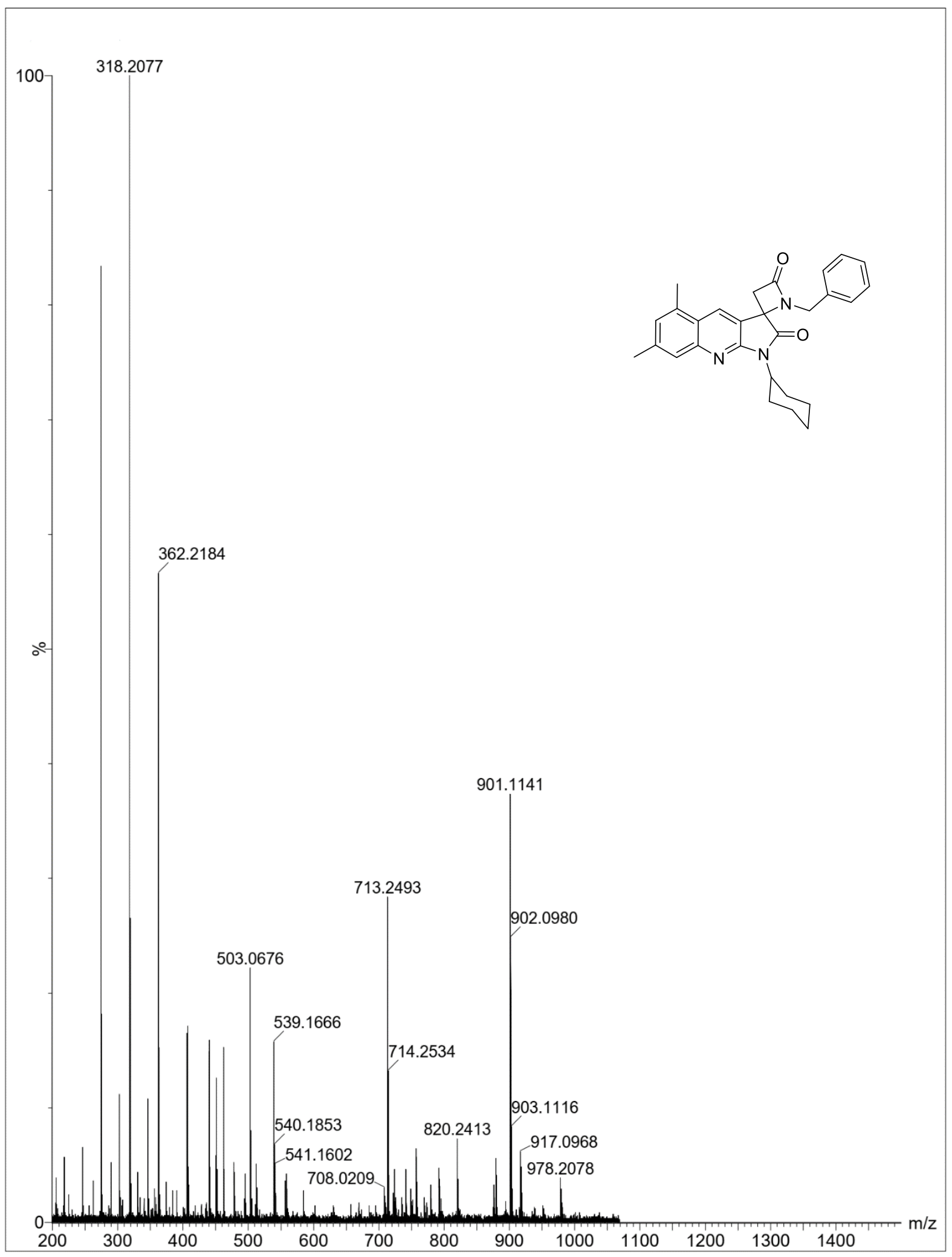

Figure S179: ESI-MS (+) of compound $\mathbf{6 s}$ with formula $\mathrm{C}_{28} \mathrm{H}_{29} \mathrm{~N}_{3} \mathrm{O}_{2}$ and $[2 \mathrm{M}+\mathrm{Na}]^{+} 901.1141$ 

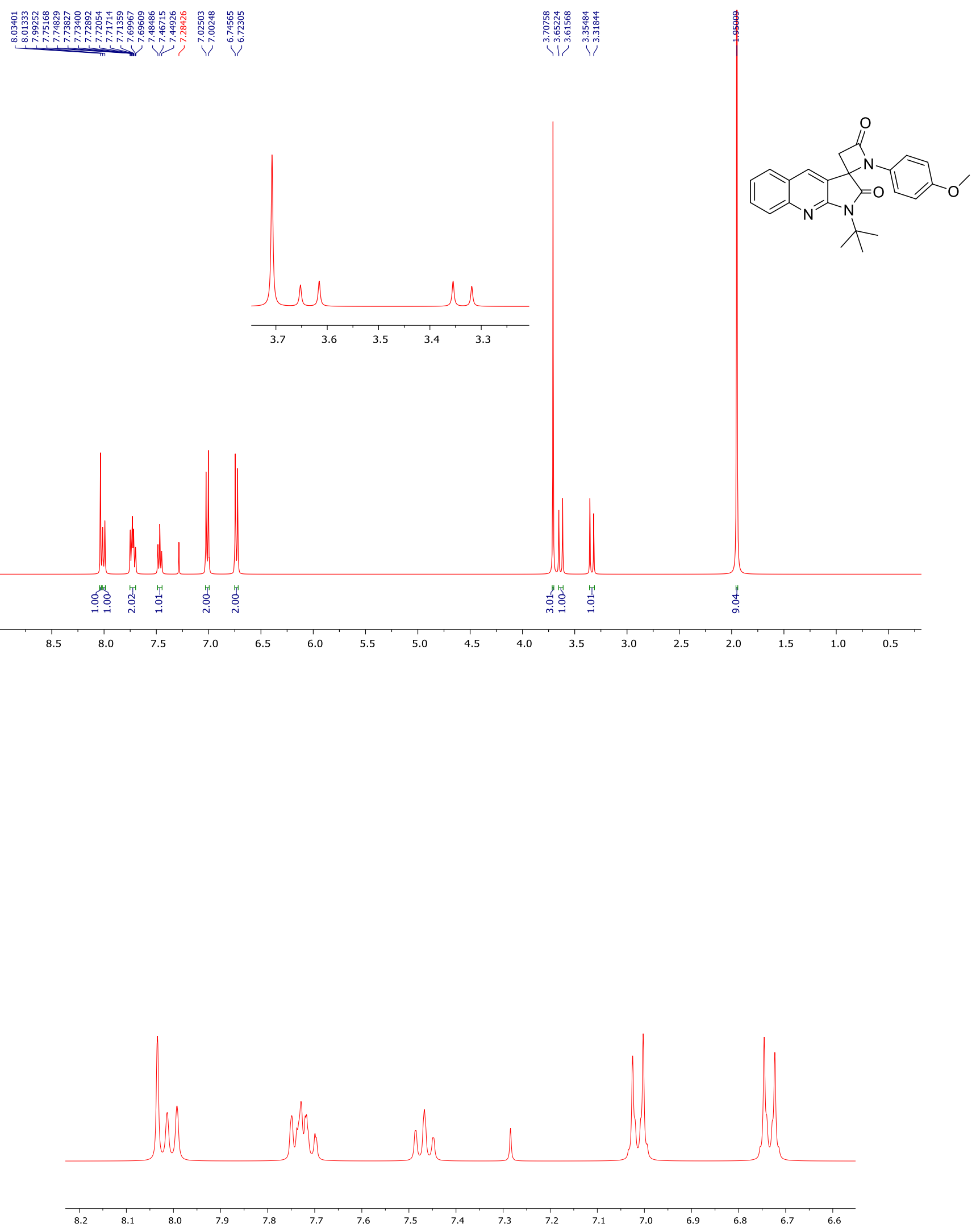

Figure S180: ${ }^{1} \mathrm{H}-\mathrm{NMR}$ of compound $\mathbf{6 t}\left(400 \mathrm{MHz}, \mathrm{CDCl}_{3}\right)$ 


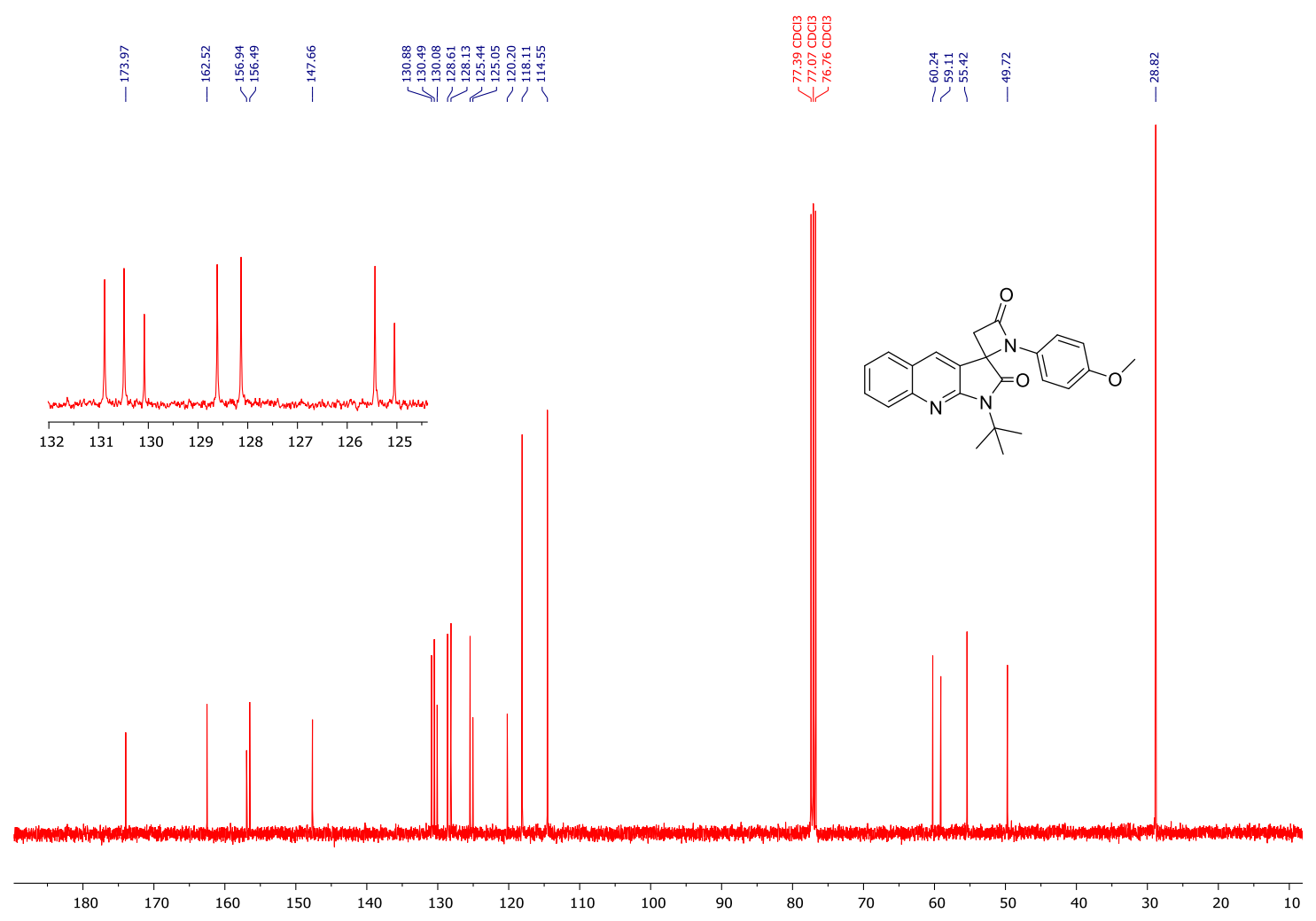

Figure S181: ${ }^{13} \mathrm{C}-\mathrm{NMR}$ of compound $\mathbf{6 t}\left(100 \mathrm{MHz}, \mathrm{CDCl}_{3}\right)$

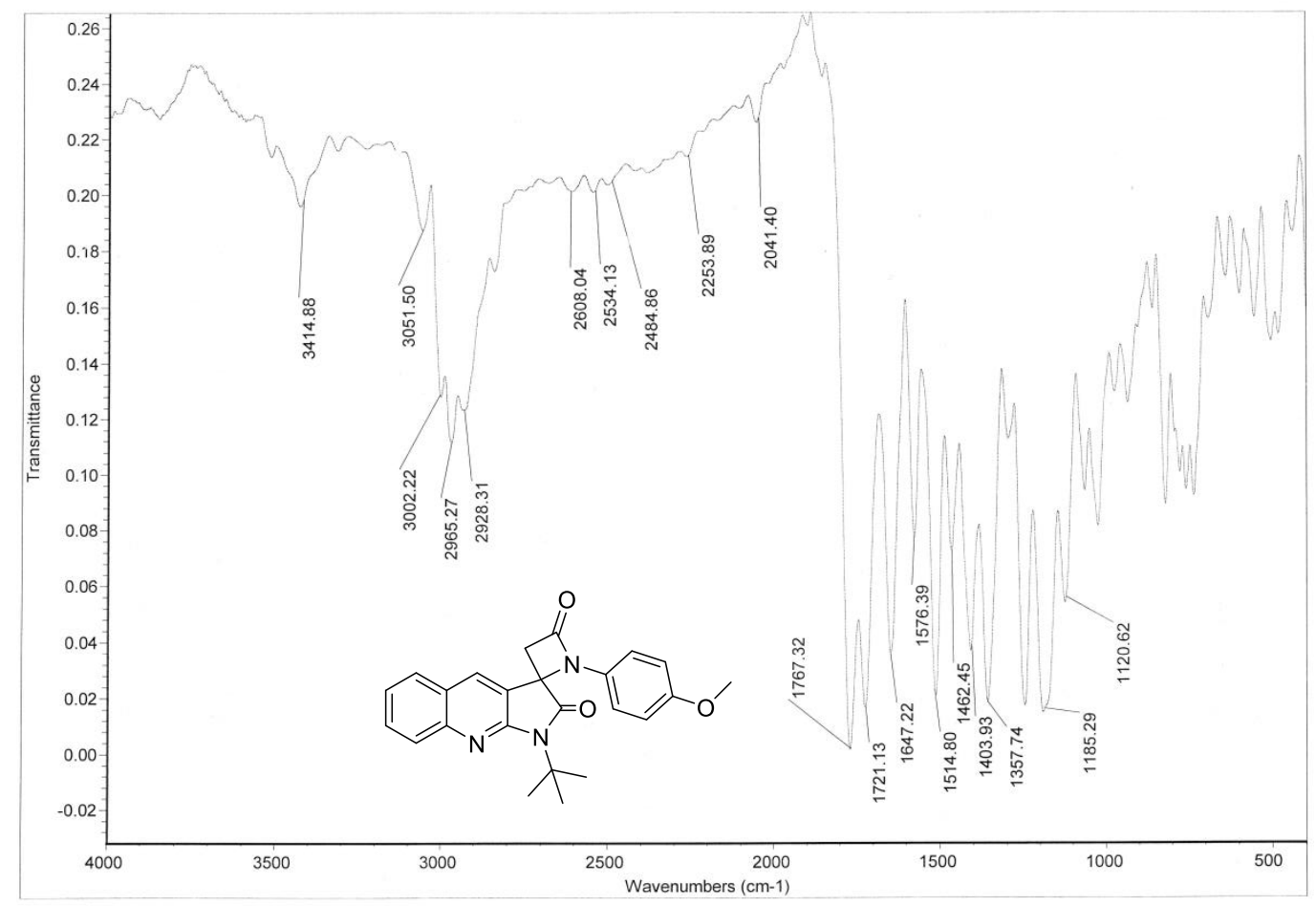

Figure S182: IR of compound $6 \mathrm{t}\left(\mathrm{KBr}, \mathrm{cm}^{-1}\right)$ 


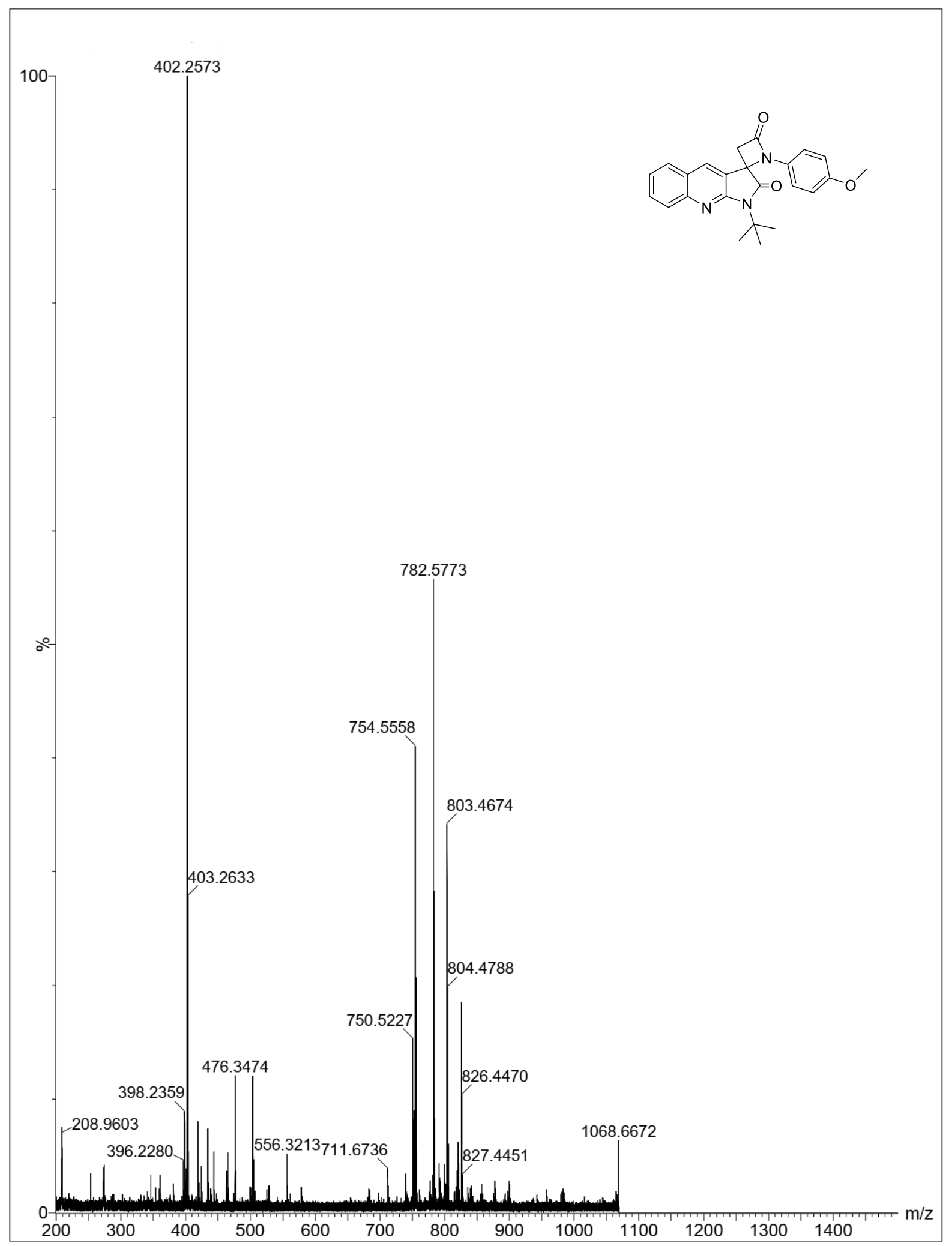

Figure S183: ESI-MS (+) of compound 6t with formula $\mathrm{C}_{24} \mathrm{H}_{23} \mathrm{~N}_{3} \mathrm{O}_{3}$ and $[\mathrm{M}+\mathrm{H}]^{+} 402.2573$. 


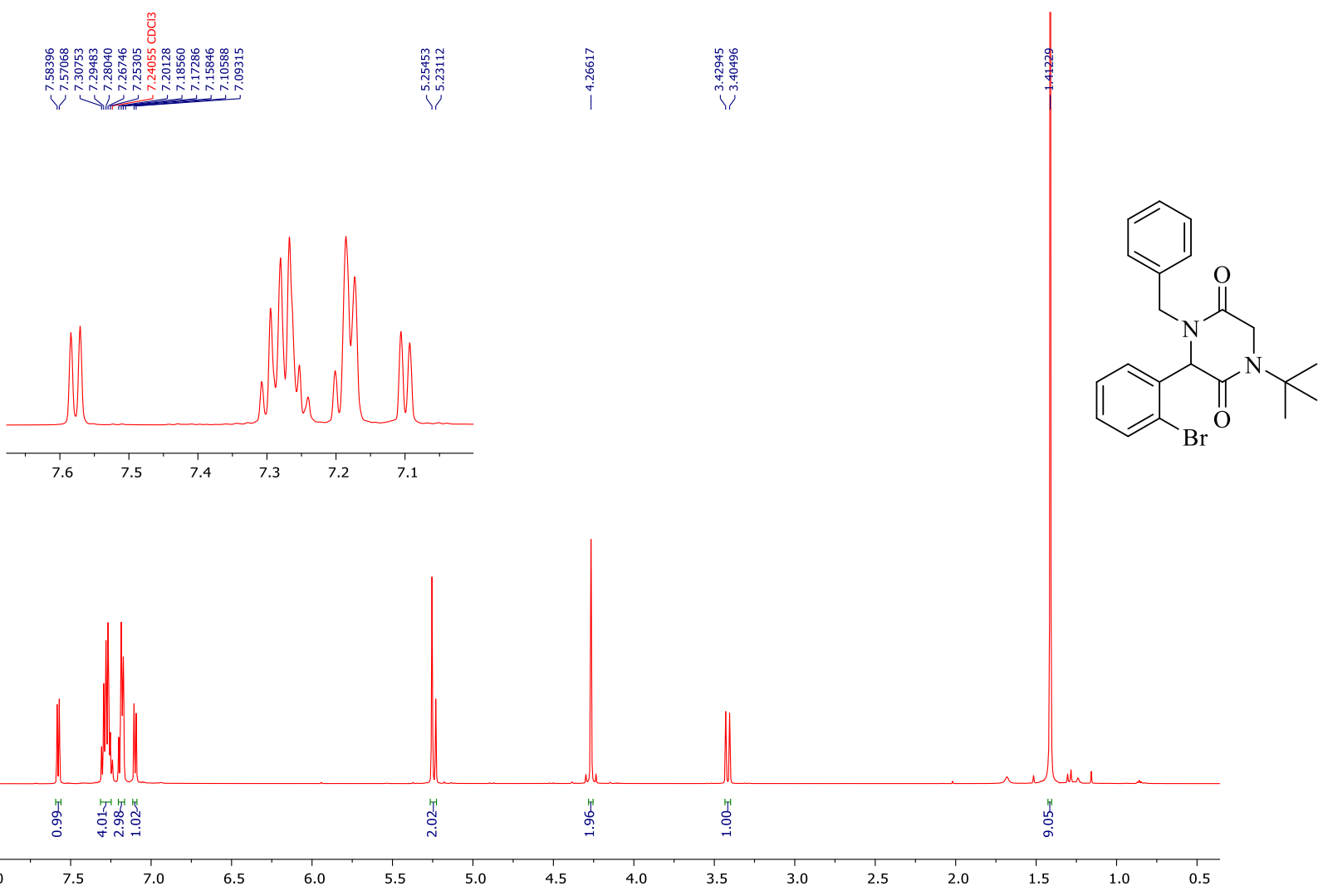

Figure S184: ${ }^{1} \mathrm{H}-\mathrm{NMR}$ of compound $12\left(600 \mathrm{MHz}, \mathrm{CDCl}_{3}\right)$

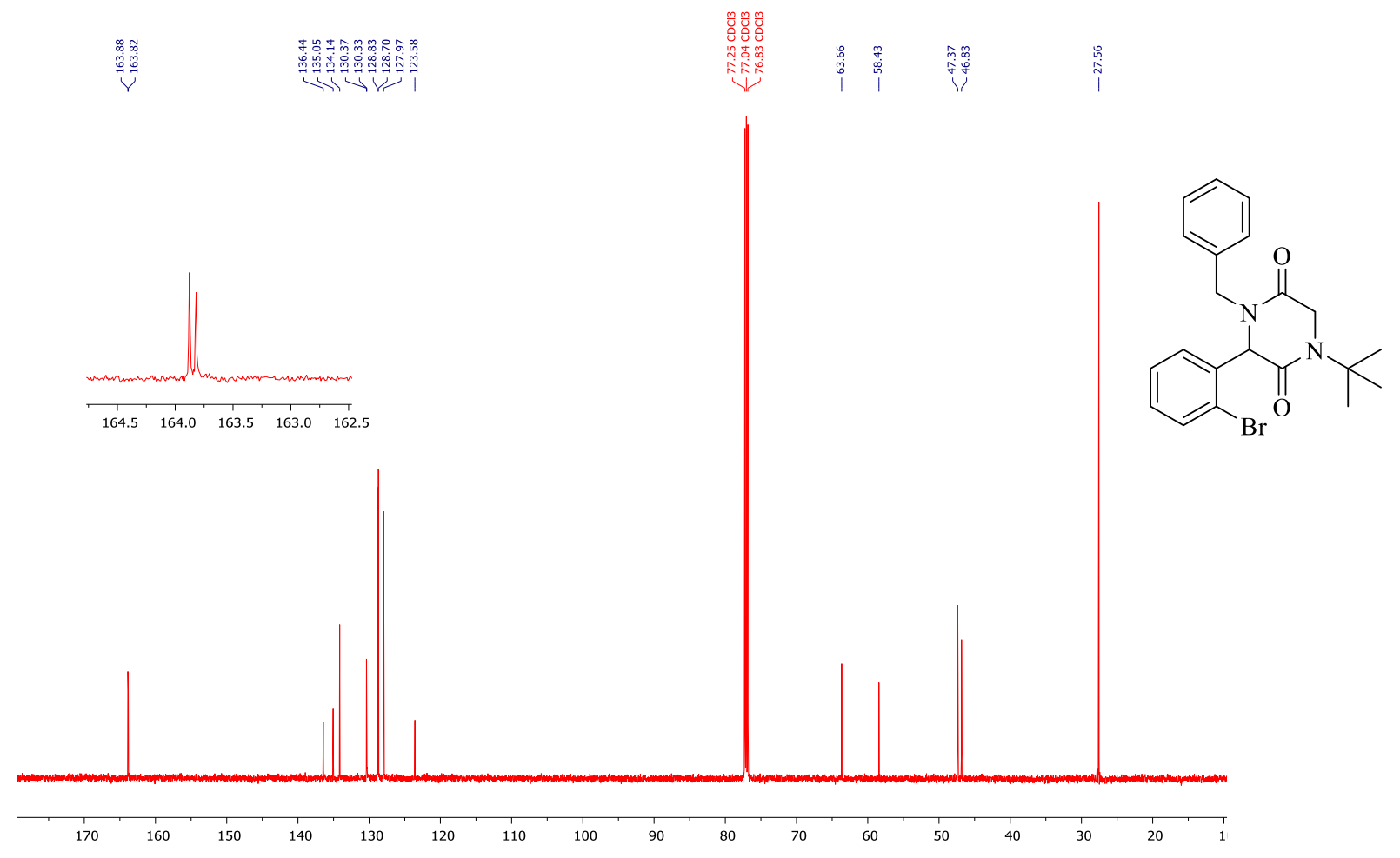

Figure S185: ${ }^{13} \mathrm{C}-\mathrm{NMR}$ of compound $12\left(150 \mathrm{MHz}, \mathrm{CDCl}_{3}\right)$ 


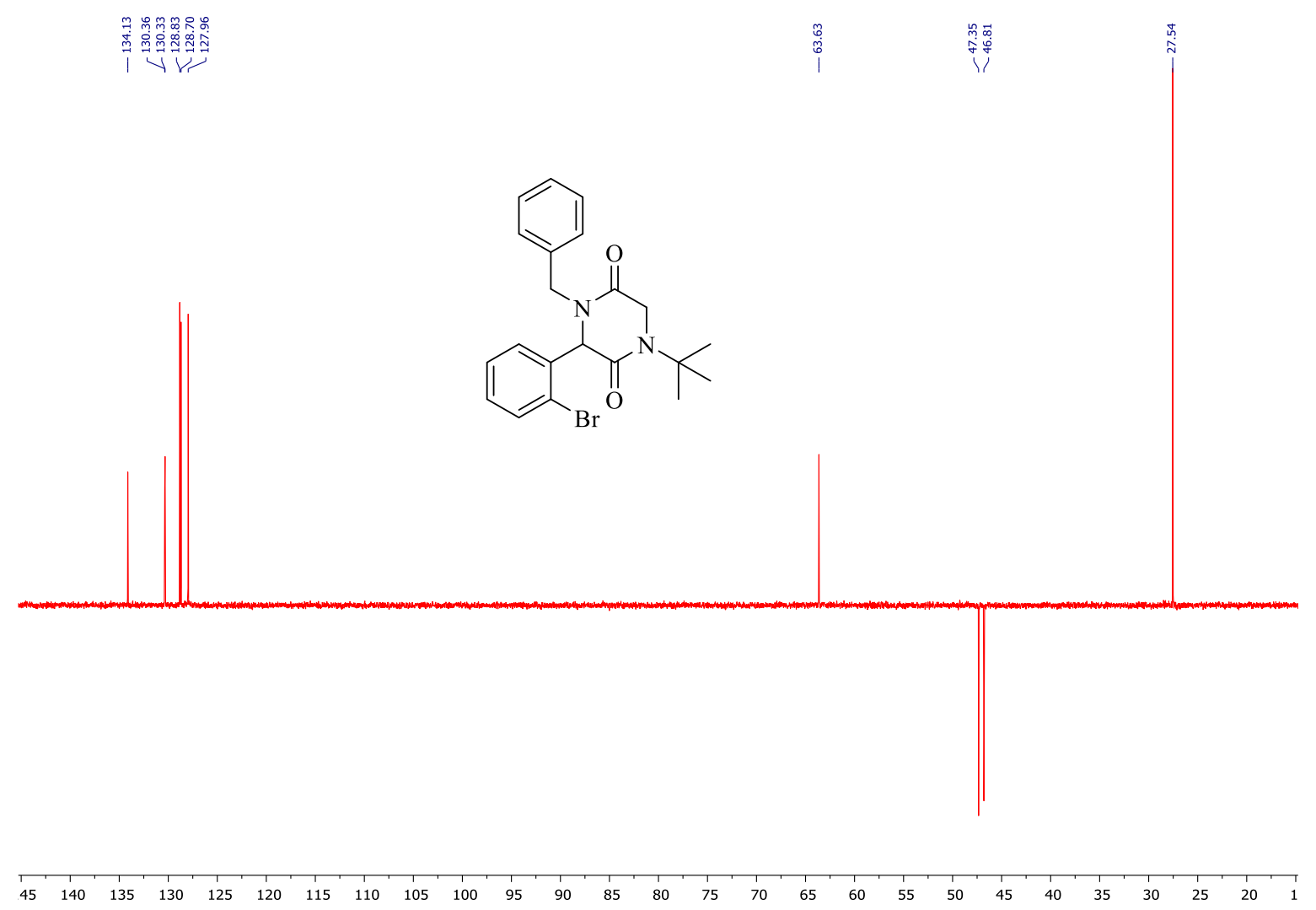

Figure S186: ${ }^{13} \mathrm{C}-\mathrm{NMR}$ (DEPT 135) of compound $12\left(150 \mathrm{MHz}, \mathrm{CDCl}_{3}\right)$ 

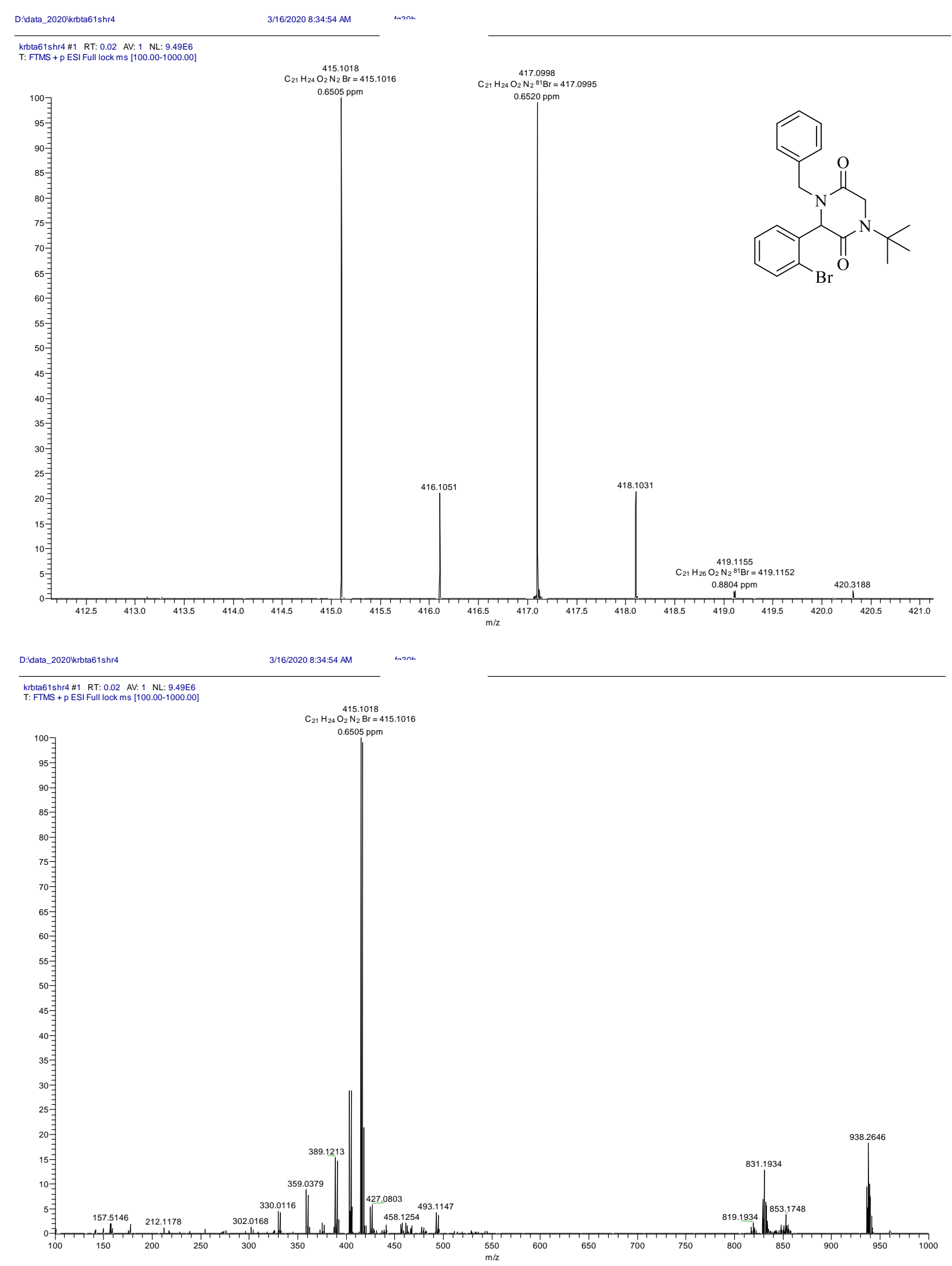

Figure S187: HRMS-ESI of 12 with formula $\mathrm{C}_{21} \mathrm{H}_{23} \mathrm{BrN}_{2} \mathrm{O}_{2}$ and $[\mathrm{M}+\mathrm{H}]^{+} 415.1016$. 


\section{X-ray Crystallographic Analysis for Compound 6b}

\begin{tabular}{|c|c|}
\hline Crystal data & $\mathbf{6 b}$ \\
\hline Chemical formula & $\mathrm{C}_{24} \mathrm{H}_{23} \mathrm{~N}_{3} \mathrm{O}_{2}$ \\
\hline $\mathrm{Mr}$ & 385.45 \\
\hline Crystal system, space group & Monoclinic, $\mathrm{P} 2{ }_{1} / \mathrm{c}$ \\
\hline Temperature $(\mathrm{K})$ & 296 \\
\hline$a, b, c(\AA)$ & $9.8585(6), 9.5244(6), 21.7395(13)$ \\
\hline$\beta\left(^{\circ}\right)$ & $99.223(2)$ \\
\hline $\mathrm{V}\left(\AA^{3}\right)$ & $2014.9(2)$ \\
\hline $\mathrm{Z}$ & 4 \\
\hline Radiation type & Mo K $_{\alpha}$ \\
\hline$\mu\left(\mathrm{mm}^{-1}\right)$ & 0.08 \\
\hline Crystal size (mm) & $0.37 \times 0.25 \times 0.18$ \\
\hline \multicolumn{2}{|l|}{ Data collection } \\
\hline Diffractometer & Bruker APEX-II CCD \\
\hline Absorption correction & Multi-scan \\
\hline & SADABS \\
\hline $\mathrm{T}_{\min }, \mathrm{T}_{\max }$ & $0.687,0.745$ \\
\hline $\begin{array}{l}\text { No. of measured, independent and } \\
\text { observed }[\mathrm{I}>2 \sigma(\mathrm{I})] \text { reflections }\end{array}$ & $28893,4289,3063$ \\
\hline $\mathrm{R}_{\text {int }}$ & 0.055 \\
\hline$(\sin \theta / \lambda) \max \left(\AA^{-1}\right)$ & 0.634 \\
\hline \multicolumn{2}{|l|}{ Refinement } \\
\hline $\mathrm{R}\left[\mathrm{F}^{2}>2 \sigma\left(\mathrm{F}^{2}\right)\right], w R\left(\mathrm{~F}^{2}\right), \mathrm{S}$ & $0.048,0.117,1.03$ \\
\hline No. of reflections & 4289 \\
\hline No. of parameters & 265 \\
\hline $\mathrm{H}$-atom treatment & $\mathrm{H}$-atom parameters constrained \\
\hline$\Delta \rho_{\max }, \Delta \rho_{\min }\left(\mathrm{e} \AA^{-3}\right)$ & $0.21,-0.17$ \\
\hline
\end{tabular}




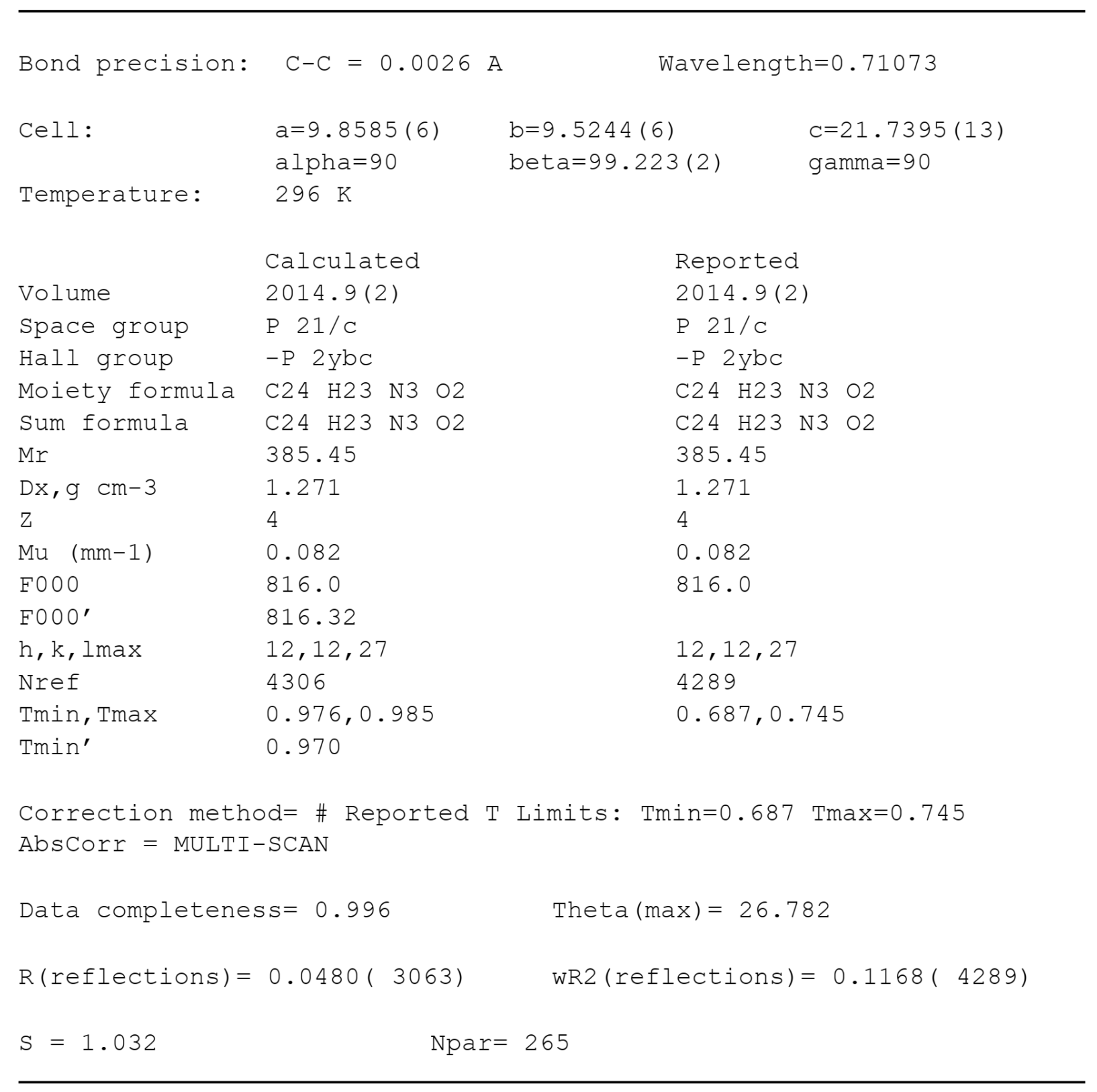




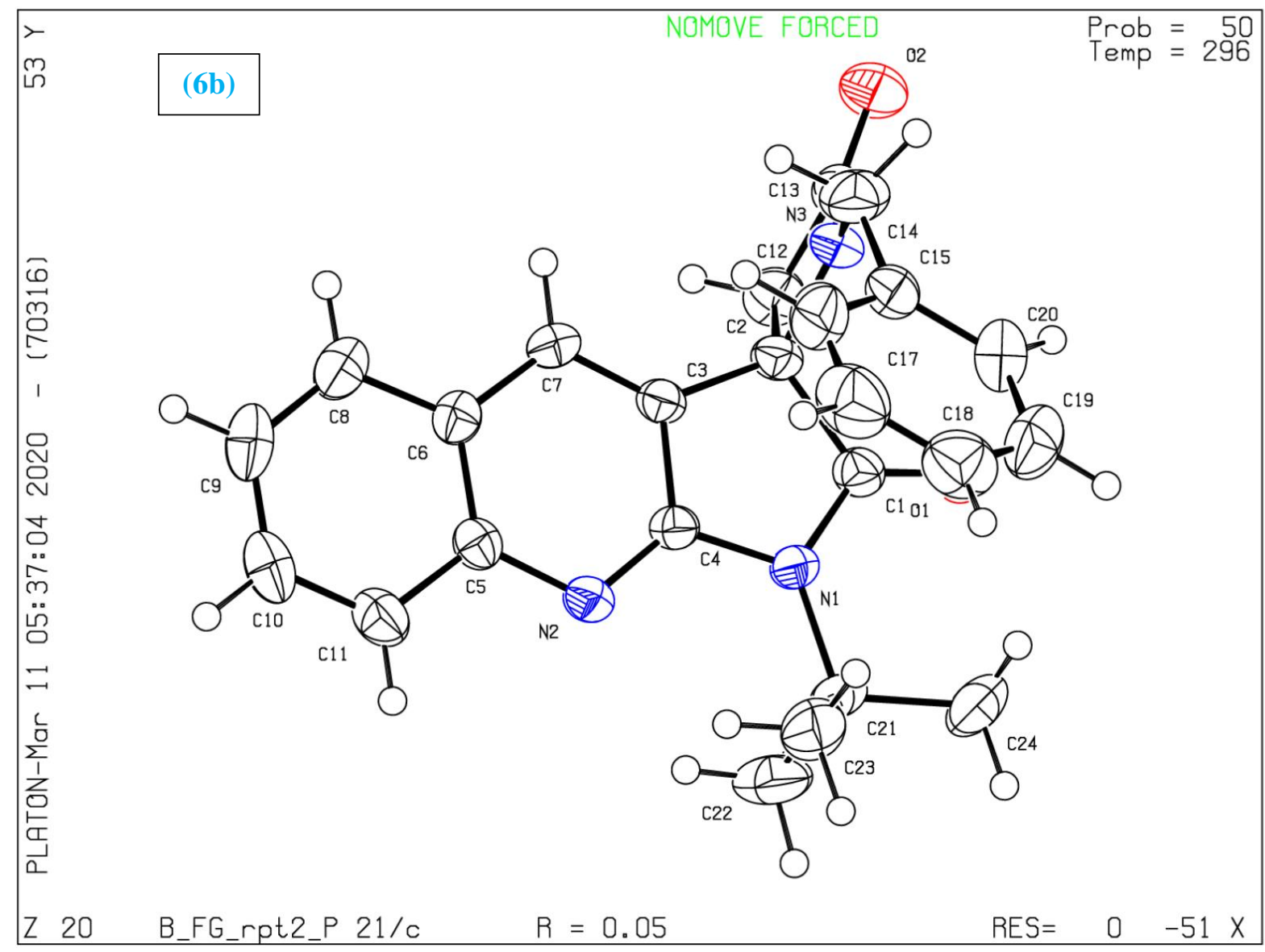

Figure S188: Crystal structures of $\mathbf{6 b}$ whose displacement ellipsoids were drawn at the $50 \%$ probability level

For X-ray measurements, single crystals of $\mathbf{6 b}$ was mounted on a MiTeGen loop with grease and examined using a Bruker D8 Venture APEX diffractometer equipped with a Photon 100 CCD area detector at 296 (2) K using graphite-monochromated Mo-K $\alpha$ radiation $(\lambda=0.71073 \AA$ ). Data were collected using APEX-II software, ${ }^{\mathrm{S} 1}$ integrated using SAINT ${ }^{\mathrm{S} 2}$ and corrected for absorption using a multi-scan approach (SADABS). ${ }^{\text {S3 }}$ Final cell constants were determined from full leastsquares refinement of all observed reflections. The structure was solved using intrinsic phasing (SHELXT). ${ }^{\text {S4 }}$ All non-hydrogen atoms were located in subsequent difference maps and refined anisotropically with SHELXL- 2016/6. Hydrogen atoms were added at calculated positions and refined with a riding model. The structure has been deposited with the CCDC (CSD deposition numbers 1989593). 


\section{References}

(S1) APEX-II, Bruker AXS, Madison, WI, USA.

(S2) SAINT, Bruker AXS, Madison, WI, USA.

(S3) SADABS, Bruker AXS, Madison, WI, USA.

(S4) Sheldrick GM. SHELXT: Integrating space group determination and structure solution. Acta Crystallogr, Sect A Found Adv. 2015, A 71, 3-8. 